\section{Prize Papers}

\section{Herbert Jasper Prize}

\section{Changes in Corticopsinal Excitability in Reaction Time and Self-paced Movements}

Robert Chen, Zaneb Yaseen, Leonardo Cohen, Mark Hallett (Bethesda, Maryland)

We used trascranial magnetic stimulation (TMS) to study the time course of corticospinal excitability before and after brisk thumb abduction movements, either in a simple reaction time (RT) paradigm or self-paced. Premovement increase in corticospinal excitability began at about $20 \mathrm{msec}$ earlier for selfpaced compared to simple RT movements. For both simple RT and self-paced movements, there was a first period of increased excitability from 0 to $100 \mathrm{msec}$, followed by a second period from 100 to $160 \mathrm{msec}$ after EMG offset. Corticospinal excitability was decreased from about 500 to $1000 \mathrm{msec}$ after EMG offset for both types of movements. Ours results show that motor preparation that begins 1.5 to $2 \mathrm{sec}$ prior to self-paced movement occurs upstream of the corticospinal neurons activated by TMS. The first phase of increased corticospinal excitability may be due to activity of motor cortex neurons subthreshold for activating spinal motor neurons, and the second phase may reflect a subthreshold second agonist burst. The period of decreased corticospinal excitability after movement corresponds to the onset of event-related synchronization (ERS) of electroencephalographic signals in the $20 \mathrm{~Hz}$ band, and supports the hypothesis that ERS may be related to an inactive, idling state of the motor cortex.

\section{The K.G. McKenzie 1st Prize in Basic Neuroscience Research}

\section{Vascular Endothelial Growth Factor (VEGF) Expression and Secretion in Astrocytomas is Dependent on Activation of the Ras Pathway}

MM Feldkamp, J Rak, N Lau, RS Kerbel, A Guha (Toronto, Ontario)

Prior work has identified vascular endothelial growth factor (VEGF) as the major angiogenic factor in normal development and in the progression of numerous tumors, including glioblastoma multiforme (GBM). Previous work has identified a link between oncogenic Ras mutations and increased VEGF production in non-astrocytic cell culture experiments. As GBMs do not harbor oncogenic Ras mutations, we sought to elucidate the relationship between Ras and VEGF in astrocytoma cell lines, both under normoxic and hypoxic conditions.

VEGF secretion into conditioned medium was measured using a quantitative ELISA assay. Ras pathway activity was inhibited genetically by transfecting cells to express the dominant negative Ras-N17, as well as pharmacologically using the farnesyl transferase inhibitor (FTI) L-744,832. Cells were ren- dered hypoxic in a sealed hypoxia chamber.

ELISA confirmed that astrocytoma cell lines secrete very high levels of VEGF. Expression of the constitutively-activated mutant epidermal growth factor receptor p $40^{\mathrm{EGF}-\mathrm{R}}$ resulted in a $55 \%$ increase in VEGF secretion. Inhibition of the Ras pathway using Ras $-\mathrm{N} 17$ resulted in $30-75 \%$ reductions in VEGF secretion. U87 and U373 cells treated with $20 \mu \mathrm{M}$ L-744,832 for 24 hours demonstrated a reduction in cellular proliferation as well as demonstrating a reduction in VEGF secretion of $15.2-43.0 \%$ (VEGF secretion normalized to number of viable cells present). While hypoxia consistently increased VEGF secretion, this hypoxic induction was severely reduced or even abolished by L744,832 and Ras-N17.

This study demonstrates that increased Ras pathway activity in astrocytoma cells results in increased VEGF secretion, which is known to be critical for tumor growth through the induction of angiogenesis. Ras pathway inhibition not only reduces cell proliferation, but also reduces VEGF secretion. Thus, novel molecularly-targeted Ras pathway inhibitors, such as FTIs, hold much promise in the future management of GBMs.

\section{The K.G. McKenzie 2nd Prize in Basic Neuroscience Research}

\section{Exposure of Dopaminergic Cells to DGNF Prior to Transplantation Enhances Graft Survival and Sensorimotor Behavioural Recovery}

\section{Mehta (Halifax, Nova Scotia)}

Although currently there are a number of medical and surgical treatment options for Parkinson's disease (PD), none of these are curative. The success of fetal transplantation in both the parkinsonian rodent and primate model has led to clinical trials in parkinsonian patients. In the parkinsonian rodent model, fetal grafting has improved rotational bias, however graft derived benefit for more complex sensorimotor deficits like forelimb akinesia has been limited. Demonstration of improvement in these tests is relevant as neural grafting attempts to clinically ameliorate motor symptoms.

We postulated that "priming" of fetal dopaminergic neurons in a hibernation media rich in glial derived neurotrophic factor (GDNF) prior to transplantation into rats with unilateral 6hydroxydopamine lesions may improve complex sensorimotor activity. Animals that received 400,000 cells exposed to GDNF demonstrated significant improvement of contralateral forelimb function and had improvement of rotational bias faster than animals that received cells not exposed to GDNF. Increasing the number of implanted cells to 800,000 also exposed to GDNF did not result in any significant increase in functional recovery.

As neural grafting procedures into the nervous system evolve and genetically engineered cells replace fetal tissue, crucial questions about cell number and trophic regulation will need to be addressed. This study demonstrates that the grafting of 400,000 cells exposed to GDNF prior to transplantation has a beneficial effect in the restoration of complex sensorimotor behaviour. 


\section{The K.G. McKenzie Prize in Clinical Neuroscience Research}

\section{Improving the Appropriateness of Carotid Endarterectomy: Results of a Prospective City-Wide Study}

John H. Wong, J. Max Findlay, Tracey B. Lubkey, Maria Suarez-Almazor (Edmonton, Alberta)

Background: In light of previously reported concerns regarding carotid endarterectomy (CEA) utilization in our city, our goal was to determine the influence of a prospective audit and educational campaign on the performance of CEA with respect to the surgical appropriateness and complication frequency.

Methods: Results of our previous audit were supplied to participating surgeons. For the 184 patients undergoing CEA over 18 months in our city, based upon clinical practice guidelines in conjunction with blinded remeasurement of their carotid angiograms, the appropriateness of surgery was classified as appropriate for patients with symptomatic carotid stenosis $70 \%$, uncertain for those with symptomatic stenosis $<70 \%$ or asymptomatic stenosis $60 \%$, and inappropriate for those with asymptomatic carotid stenosis $<60 \%$ or if they were neurologically or medically unstable preoperatively.

Results: When compared with our previous audit, we found that the rate of appropriate CEAs had improved from $33 \%$ to $48 \%$ in the present study $(\mathrm{P}=0.01)$, uncertain indications did not change significantly from $49 \%$ to $47 \%(\mathrm{P}=0.72)$, and inappropriate indications dropped from $18 \%$ to $5 \%(\mathrm{P}=0.00003)$. Stroke or death rates of $6.4 \%$ and $2.7 \%$ were found in the symptomatic and asymptomatic subgroups, respectively.

Conclusions: The present study found a significant increase in the appropriate use of CEA and a dramatic decrease in inappropriate operations. Surgical benefit increased in our asymptomatic patients due to fewer complications in this subgroup. Audits which identify problems with procedure utilization, followed by educational campaigns and continued surveillance, can improve the appropriateness of CEA.

\section{The President's Prize}

\section{Clinical and Electrophysiological Features of Children with Mitochondrial Disorders and Epilepsy}

\section{Lynette Sadleir (Toronto, Ontario)}

Background: Mitochondrial diseases are an important cause of neurologic disease in children. The electroclinical features of seizures associated with mitochondrial diseases are not well described.

Methods: Charts of children with mitochondrial diseases and epilepsy were selected from the records of the Biochemical Diseases Division of a tertiary care children's hospital. Interictal electroencephalograms and video-EEG recordings of seizures were examined.

Results: Spasms occurred in nine patients, who subsequently developed other seizure types. Five patients had only partial seizures, with or without generalisation. Two patients had seizures that were difficult to classify. Eight of the nine patients with spasms had at least one normal serum lactate level. Serum lactates were consistently elevated and were substantially higher in patients with partial seizures only.

Discussion: Infantile spasms are an important seizure type in children with mitochondrial diseases and epilepsy. Patients with unexplained spasms, particularly those with delayed neurologic development, should be investigated in detail for mitochondrial diseases. The normal lactate levels observed frequently in the patients with spasms, emphasises the low sensitivity of this investigation in screening for mitochondrial diseases.

\section{Francis McNaughton Memorial Prize}

\section{O6-methylguanine-DNA Methyltransferase Levels Vis-à-vis Clinical Response to 1,3-(2-chloroethyl)-1-Nitrosourea Based Chemotherapy in Glioma Patients}

Zhong-Ping Chen, Daniel Yarosh, Donatella Tampieri, Gérard Mohr, Lawrence C. Panasci (Montreal, Quebec) and Applied Genetics Inc. (DY) (Freeport, New York)

Background: It has been documented in tumor cell lines that O6-methylguanine-DNA methyltransferase (MGMT) plays an important role in chloroethylnitrosourea (CENU) resistance.

Methods: We evaluated MGMT expression in 22 glioma specimens by immunofluorescence assay and compared the results with clinical response of the patients to BCNU based chemotherapy.

Results: Eight samples had no detectable MGMT, while other samples had from 9989 to 982401 molecules/nucleus. Twelve patients (effective group) demonstrated decreasing tumor size or stable, while in 10 patients the tumor continuous growth during chemotherapy (progression group). There was no correlation between MGMT levels and either the survival time ( $r=0.04, p=0.8595$ ) or TTP (the median time to progression) $(\mathrm{r}=0.107, \mathrm{p}=0.6444)$. However, in glioblastoma multiformas (GBMs), the TTP of Mer+ (MGMT $>60000$ molecules/nucleus) patients was shorter than that of Mer- (MGMT $<60000$ molecules/nucleus) patients $(t=2.04, p=0.049)$. The MGMT levels were much higher in progressive GBMs than in effective GBMs $(\mathrm{t}=-2.26, \mathrm{p}=0.029)$.

Conclusions: This study confirmed that MGMT plays an important role in BCNU resistance in gliomas, especially in GBMs. MGMT positive tumors are usually but not always resistant to CENUs. However, MGMT negative tumor are not always sensitive to CENUs, suggesting that other factors may also be important. 


\section{Oral Presentations}

\section{A. CEREBROVASCULAR DISEASE}

\section{A-01}

\section{CGRP in Migraine Pathophysiology: A Therapeutic Role?}

Y Knight, PJ Goadsby, L Edvinsson (London, United Kingdom)

Calcitonin gene-related peptide (CGRP) is the most potent known vasodilator and is released during the headache phase of migraine. It is known to be released into the vasculature from activated trigeminal nerve terminals which it can then re-activate; as such CGRP is a marker for trigeminovascular activity.

CGRP may have a role in migraine through its potent cranial vasodilatory effects or by an action on trigeminal nerve activity. Both of these actions are targeted by 5HT1B/1D agonist drugs like sumatriptan, of which two are avitriptan and CP122,288. Both avitriptan and CP122,288 have strong binding affinities for 5 HTIB/ID receptors, but only avitriptan has shown to be effective in aborting migraine. Comparison of the effects of avitriptan and CP122,288 on CGRP released into cat jugular vein blood as elicited by stimulation of the superior sagittal sinus (SSS) shows that only avitriptan affects CGRP release. When considered with other findings that sumatriptan and DHE also affect CGRP release and abort migraine it is suggested that blockade of CGRP release could be a new non-5HT based option for migraine therapaeutics.

\section{A-02}

Studies to Investigate the Minimum Effective Dose of Licostinel (ACEA 1021) in a Rat Model of Transient Focal Cerebral Ischemia

D. Xue, B. K. Miyazaki, X. Cheng, G. Daniell, R. M. Woodward (Irvine, California)

Background: Licostinel is a high potency NMDA receptor glycine site antagonist and moderate potency AMPA receptor antagonist. Previous studies indicate that licostinel has neuroprotective effects in a variety of rat focal ischemia models. In these experiments, however, the drug was tested at moderate or high doses. The current study was designed to investigate the minimum effective dose of licostinel and to simultaneously measure the steady-state plasma level of the drug associated with this dose.

Methods: A total of 129 Fischer-344 rats were subjected to a $2 \frac{1}{4}$-hour ischemia followed by reperfusion. Licostinel was administered as an i.v. bolus followed by a $22 \mathrm{~h}$ i.v. infusion. The bolus was given immediately after ischemia. Plasma samples were taken at $4 \mathrm{~h}$, and levels of licostinel were assayed by LC / MS. Infarct volumes were assessed in brain slices by TTC staining $2 \mathrm{~h}$ after the end of the infusion. Licostinel was tested at five doses: $5+3.5,2.5+1.75,1.25+0.875,0.625+0.438$, and $0.313+0.219(\mathrm{mg} / \mathrm{kg}$ bolus $+\mathrm{mg} / \mathrm{kg} / \mathrm{h}$ infusion $)$.

Results: The mean reductions in cortical infarct volume were: $48,72,66,46$, and $17 \%$, respectively. All but the lowest dose produced significant protection (Dunnett's test $p<0.05$ ). The steady-state plasma levels of licostinel at the five doses were approximately: $20 ; 10,4,2$ and $1 \mathrm{mg} / \mathrm{ml}$, respectively. The core body temperatures in rats dosed at $5+3.5$ and $2.5+1.75$ were slightly reduced $\left(<1.8^{\circ} \mathrm{C}\right)$ compared to the vehicle group. There were no significant temperature effects at lower doses. There were no obvious behavioral effects at any dose tested.

Conclusions: We conclude that the minimum effective dose of licostinel in this model is a $0.625 \mathrm{mg} / \mathrm{kg}$ i.v. bolus +0.438 $\mathrm{mg} / \mathrm{kg} / \mathrm{h}$ i.v. infusion, and that the steady-state total plasma level associated with this dose is $\sim 2 \mathrm{mg} / \mathrm{ml}$. These values will be useful for targeting plasma levels in ongoing clinical trials for stroke.

\section{A-03}

Neuroprotection and Thrombolysis: Alone or in Combination in A Focal Embolic Model of Cerebral Ischemia

\section{A. Shuaib, Y Yang, Q. Li (Edmonton, Alberta)}

Background: A significant increase in the development of cerebral hemorrhage is a major limitation with the use of thrombolysis. It is possible that the use of 'neuroprotectant medication' may prolong the time in which thrombolysis could be offered.

Methods: To test this hypothesis, we used a focal model of middle cerebral artery (MCA) ischemia and evaluated the efticacy of urokinase (5000 units per $\mathrm{kg}$ ) infusion at two, three and six hours. In a second series of experiments, topiramate (40 $\mathrm{mg}$ per $\mathrm{kg}$ ) was used as a single medication at two hours in set of animals and compared to topiramate at two hours combined with urokinase at 6 hours. All experiments were compared with a group of saline treated animals. Neurological behavior tests were done at 2 hours and 24 hours. Animals were sacrificed at 24 hours and the percentage of infarct volume was determined. The MCA was occluded by exogenous clot with blood drawn from the MCA and treated with thrombin prior to re-infusion.

Results: There was a significantly better neurological outcome at 24 hours in the animals treated with either medication compared to controls. The group treated with urokinase alone at 6 hours showed the least amount of protection. The volume of the infarction in the control saline treated group was 54 ' $191+1-$ $9.04] \%$. In the group treated with urokinase at 2 hours this significantly decreased to $18.38 \%$ [ $+/-8.5]$. The use of topiramate at 2 hours showed a decrease in the infarction to $7.6[+/-3.39] \%$ of the hemisphere. Treatment with urokinase at six hours after the occlusion showed a trend towards protection with a reduction to $31.9[+/-14.14] \%$ of the ipsilateral cerebral hemisphere. Finally, when topiramate was given at two hours and urokinase was given at 6 hours there was significant protection and a decrease in the infarct size to $8.9[+/-5.07] \%$ of the hemisphere with no mortality and excellent functional recovery at 24 hours.

Conclusion: Our study shows that the volume of the infarction can be significantly decreased with intraarterial thrombolysis and early treatment with topiramate, urokinase can safely and effectively be used for up to 6 hours after the insult. 


\section{A-04}

Neuroprotection by Two-Hour Delayed Administration of Topiramate in the Middle Cerebral Artery Embolization Model of Rat

\section{Y. Yang, A. Shuaib, Q. Li (Edmonton, Alberta)}

Background: Topiramate (TPM) has shown effects on suppression of voltage-sensitive $\mathrm{Na}+$ channels and non-NMDA glutamate receptors as well as enhancement of GABA-mediated inhibition. It may theoretically have a potential effect of neuroprotection of cerebral ischemia. This hypothesis was examined in a middle cerebral artery (MCA) embolization model of rat.

Methods: All animals were subjected to focal ischemia by introduction of autogenous thrombus into the right MCA.The animals in low-dose group were received TPM at a single dose of $20 \mathrm{mg} / \mathrm{kg}$ (i.p.; $\mathrm{n=6}$ ) and high-dose group at a dose of 40 $\mathrm{mg} / \mathrm{kg}$ (i.p.; $\mathrm{n}=10$ ) while the control animals given with the same volume of vehicle (i.p.; $n=6$ ) $2 \mathrm{~h}$ after injection of thrombus. The neurologic behavior was scored at $2 \mathrm{~h}$ and $24 \mathrm{~h}$ following the ischemic insult. The animals were sacrificed $24 \mathrm{~h}$ afterwards and the coronal sections of brains were stained with $2 \%$ TTC for estimation of the percentage of infarct volume.

Results: Administration of TPM significantly improved $24 \mathrm{~h}$ neurologic behavior scores (low dose, 1.75 0.5 ; high lose, $1.17 \pm 0.41, \mathrm{p}<0.05$ for both doses) and reduced the percentage of infarct volume (low dose $22.9+/-8.9 \%, p=0.002$; high dose $7.6+1-3.4 \%, \mathrm{p}<0.001$ ) when compared to the control (infarct size $54.2+1-9.0 \%$; behavior score $2.67 \pm 0.52$ ). Treatment of TPM at higher dose induced more neuroprotection than that at lower dose $(\mathrm{p}<0.05)$.

Conclusion: TPM showed neuroprotective effect in a useand dose-dependent fashion when used $2 \mathrm{~h}$ after MCA embolization of rat.

\section{A-05}

\section{Doppler Diagnosis of Carotid Dissections}

Helen Romanchuk, Carol Derksen, Ashfaq Shuaib, (Edmonton, Alberta)

Stroke can result from multiple causes. In the absence of cardiac disease, the carotid arteries are investigated with cranial CT and Triplex Doppler as the screening procedures of choice. If Doppler studies are normal, no further investigations are usually necessary. Carotid dissection is an uncommon cause of ischemic stroke, especially in the young patients. Carotid Doppler may have characteristic findings indicating a dissection. In this abstract we wish to present the Doppler and Angiography findings in 5 patients recently seen in the Doppler laboratory at the University of Alberta Hospital in Edmonton. The first patient showed a complete ICA occlusion with irregular gradual tapering. Second patient showed bilateral common carotid flaps and unilateral occlusion. Third patient had bilateral common carotid echogenic lines with extension into the right external carotid artery. Fourth patient had thin membrane noted in the distal portion of the common carotid artery and the fifth patient with
Marfan's syndrome had an aortic dissection extend into the left common carotid artery. All dissections were confirmed on cerebral angiography. We would like to present the clinical findings, Doppler images and cerebral angiography so as to increase the awareness of non-invasive diagnosis of carotid artery dissection.

\section{A-06}

\section{Calgary Stroke Program Early CT Study}

HG Karbalai, PS Kochanski, AM Buchan (Calgary, Alberta)

Purpose: We assess the predictive efficacy of early CT changes, once viewed as a relatively insensitive detection modality.

Methods: 36 patients with an MCA territory stroke (age $68.9 \pm 11.0(\mathrm{SD}), 24 \mathrm{M} / 12 \mathrm{~F})$ seen over 18 months, were evaluated by $\mathrm{CT}$ within 3 hours (mean $110 \pm 31$ minutes) of symptom onset and at 24 hours post $0.9 \mathrm{mg} / \mathrm{kg}$ t-PA infusion. The baseline CTs were retrospectively analyzed by physicians blinded to the follow-up results.

Results: A hemorrhagic conversion of the infarct, usually minor, was observed on $13(36 \%)$ patients, $9(25 \%)$ asymptomatic, and $4(11 \%)$ symptomatic. Early CT changes were seen on $29(81 \%)$ of the scans, as follows:

$\begin{array}{lll} & \begin{array}{l}\text { Infarcted } \\ (\mathrm{n}=23)\end{array} & \begin{array}{l}\text { Hemorrhagic } \\ \text { Conversion( } \mathrm{n}=13)\end{array} \\ \begin{array}{l}\text { No lesions } \\ \text { Hyperdense MCA }\end{array} & 5(22 \%) & 2(15 \%) \\ \text { Effaced: } & 6(26 \%) & 4(31 \%) \\ \text { Caudate } & 4(17 \%) & 1(8 \%) \\ \text { Lentiform } & 6(26 \%) & 4(31 \%) \\ \text { Insular ribbon } & 9(39 \%) & 7(54 \%) \\ \text { MCA cortical regions } & 12(52 \%) & 6(46 \%)\end{array}$

A trend was noticed (small sample size, $\therefore$ not significant), in that $10 / 23(43 \%)$ infarcted patients, compared to only $1 / 13(8 \%)$ hemorrhaged patients exhibited middle MCA distribution territory cortical effacement.

Conclusions: CT scans can effectively detect early ischemic lesions. Baseline insular ribbon effacement seems to be more indicative of lesions likely to undergo hemorrhagic conversion, but baseline effacement of MCA cortex lateral and superior to the insular seem to indicate that the patient, possibly due to a more distal occlusion, has bypassed this hemorrhagic susceptibility.

\section{A-07}

Factors Influencing Time from Stroke Onset to Hospital Arrival in Five Canadian Cities

A Hakim (Ottawa, Ontario), L Lebrun (Montreal, Quebec), S Phillips (Halifax, Nova Scotia), A Shuaib (Edmonton, Alberta), P Teal (Vancouver, British Columbia), V Ramsden (Saskatoon, Saskatchewan), C Temovsky, R Dhawan, E Gudaitis, K Lawrence, P Rawn, F Shannon (Mississauga, Ontario), The OPTIMISETM (Optimal Management and Intervention in Stroke Emergencies) Group 
Background: The NINDS trial demonstrated the efficacy of intravenous rt-PA in acute stroke and its introduction is anticipated soon in Canada. This therapy will be recommended for patients meeting the strict criteria used in the NINDS trial. Critical among these is that patients must be treated within three hours of stroke onset. We have examined the management of acute stroke patients in five Canadian tertiary care centers, and report here the factors which appear to be associated with time to presentation.

Methods: A retrospective chart review of stroke patients admitted to five Canadian tertiary care centers involved in stroke treatment was conducted. Stroke patients were identified by their ICD-9-CM codes on discharge. Chart abstraction for the 28 month period from September 1, 1994 to December 31, 1996 was performed by trained Nurse-Coordinators at each site. Data were entered into Microsoft Access, and analyzed using SAS software.

Results: Data were collected on 2022 stroke patients. The median time from symptom onset to hospital arrival was 3.6 hours (interquartile range $1.4-8.3$; total $n=889$ ). $34 \%$ of patients arrived within two hours of symptom onset, and $10 \%$ arrived in less than one hour. 55\% of patients arrived by ground ambulance, $24 \%$ were brought in by their families, and $9 \%$ transported themselves to hospital (total $n=1733$ ). Seven percent were transferred in from another hospital. Patients arriving by ground ambulance had the fastest arrival time with a median of 1.8 hours. A linear regression taking time from onset to arrival as the dependent variable showed that age (older patients arrived faster), method of arrival (arrival by ground ambulance fastest), level of consciousness (impaired consciousness fastest) and ataxia were significant covariates, together explaining $19 \%$ of the variance.

Conclusions: There are limitations to these data given the retrospective nature of the study. However, the results provide insight into the methods of arrival and factors associated with delayed presentation in Canadian stroke patients.

\section{A-08}

Early CT Signs of Infarction and Hemorrhagic Outcomes in the Treatment of Acute Ischemic Stroke with t-PA

\section{Roos, W. Hu, A.M. Buchan (Calgary, Alberta)}

Results from clinical trials of intravenous tissue plasminogen activator ( $t-P A$ ) for the thrombolytic canalization of occluded cerebral arteries in acute ischemic stroke ${ }^{1,2}$ indicate that, although therapy is effective, its primary complication is intracranial hemorrhage. The existence of CT hypodensity as a criterion for patient exclusion from treatment with t-PA is controversial. The Foothills Hospital is participating in a clinical trial of t-PA in which 34 patients, whose stroke onset times were clearly defined, were treated with t-PA within 3 hours of ictus. CT scans were obtained before treatment, as well as $24 \mathrm{~h}$ post treatment. Pre-treatment CT scans were analyzed retrospectively for signs of early ischemia indicating which patients are predisposed to hemorrhage. Obscuration of the basal ganglia (OBG) was seen in 17 patients, and 9 patients experienced hemorrhage ( $4 \mathrm{w} / \mathrm{OBG} ; 5 \mathrm{w} / \mathrm{O}$ OBG; $\chi^{2}=0.1511 ; \therefore \mathrm{NS}$ ). Thus, although many patients showed $O B G$ as a sign of early ischemia, it did not have any effect on the outcome of these patients with respect to hemorrhage. This suggests that OBG, as a sign of CT hypodensity, should not exclude patients from treatment with t-PA.

Reference:

ECASS, JAMA 274: 1017

NINDS, NEJM 333: 1581

\section{A-09}

\section{Intra-sagittal Sinus Urokinase for Superior Sagittal Sinus Thrombosis}

\section{T. Jeerakathil, A. Shuaib, R. Ashforth (Edmonton, Alberta)}

Background: Sagittal sinus thrombosis (SST) is an venous occlusive disease that presents with headache, seizures and venous infarction. Treatment usually consists of systemic anticoagulation $(\mathrm{AC})$ to prevent propagation of the clot. Other treatments include intra-sinus thrombolysis, and in rare cases, surgical evacuation of clot.

Methods: Two patients with SST were treated with intrasagittal sinus urokinase infusion. A catheter was introduced via the femoral veins, and advanced through the internal jugular vein and transverse sinuses, into the superior sagittal sinus. The catheter was advanced beyond the level of the clot. Patients were laced with 250000 iu of urokinase and started on an hourly infusion of 1000-2000iu of urokinase. The infusion was discontinued after 24-48 hours after demonstration of angiographic improvement in the clot.

Results: A 44-year-old female presented with sudden collapse, generalized seizure, right hemiparesis, and dysphasia. Angiogram and MRI head revealed bilateral cerebral hemorrhages and thrombosis of the posterior two-thirds of the superior sagittal sinus. Four days after presentation she was treated with thrombolysis. Repeat angiogram showed considerable thrombus resolution. The patient was discharged to a rehabilitation hospital on oral anticoagulants, with mild right sided weakness, some word finding difficulty, and mild right sided sensory loss.

A 32-year-old female with a history of paroxysmal nocturnal hemoglobinuria and aplastic anemia presented with numbness and weakness of left arm and leg. Initial CT head was normal but repeat $\mathrm{CT}$ head later that day showed bilateral intracerebral haematomas. The next day MRI head revealed superior sagittal sinus thrombosis. She was treated one day after presentation with thrombolysis. Repeat angiogram revealed almost complete resolution of thrombosis. Her course was complicated by heparin induced thrombocytopenia but she experienced mild improvement in her hemiparesis and was able to walk on discharge to a rehabilitation hospital on oral anticoagulants.

Conclusions: The outcome of these two cases suggests that local infusion of urokinase may be a promising method of treating sagittal sinus thrombosis. Significant angiographic resolution of thrombus and clinical improvement occurred.

\section{A-10}

\section{Warfarin Thromboprophylaxis is Underutilized in Atrial Fibrillation Patients}

\section{R. Leckey, S.J. Phillips, E.G. Aguilar (Halifax, Nova Scotia)}

Background: In clinical trials, warfarin reduced the risk of 
stroke in patients with atrial fibrillation (AF) by about twothirds. We hypothesized that the majority of AF patients presenting to our hospital with an ischemic stroke were not receiving optimal antithrombotic therapy.

Methods: Patients admitted to the Acute Stroke Service at the Queen Elizabeth II Health Sciences Centre during the 2 year period ending 31 December 1996 were identified from a prospective registry. We examined antithrombotic treatment of $\mathrm{AF}$ patients and compared the outcomes of AF patients with those in sinus rhythm (SR).

Results: AF was present in 92 of $722(13 \%)$ patients at the time of admission: 3 of $72(4 \%)$ patients with primary intracerebral hemorrhage, none of whom were taking warfarin at the time of their stroke; 10 of $107(9 \%)$ patients with TIA, none of whom were taking warfarin; and 79 of $540(15 \%)$ patients with ischemic stroke, of whom 7 were taking warfarin. Only 7 of 48 $(15 \%)$ patients with ischemic stroke who were known to be in $\mathrm{AF}$ before their stroke were taking warfarin at the time of admission. Three of these 7 had mitral valve disease. In 3 of the anticoagulated patients, the International Normalized Ratio was less than 2.0 . Only $9 \%$ of patients with known nonvalvular AF were taking warfarin at the time of their stroke. Total middle cerebral artery territory infarcts were twice as frequent among AF patients than in $S R$ patients $(\mathrm{P}=0.001)$. The case-fatality ratio for AF patients was double that of patients in SR $(\mathrm{P}=0.001)$. AF patients were less likely to go home $(31 \%$ vs $59 \%, \mathrm{P}=0.005)$.

Conclusions: AF patients had more severe strokes than patients in SR. Primary stroke prevention among AF patients in our community in 1994-96 was suboptimal.

\section{A-11}

Trends in Anticoagulation: Practices in the Management of Atrial Fibrillation For Stroke Prevention (1988-1995)

J.Kashmere (Saskatoon, Saskatchewan), A. Shuaib (Edmonton, Alberta)

Background: There is widespread research support for anticoagulation in patients with non-valvular atrial fibrillation (NVAF). However, it has not yet been established if these recommendations have been accepted in Canadian communities.

Methods: We reviewed 346 charts of people with NVAF at a tertiary care hospital in Saskatoon, Canada. We examined the management trends to see how they have changed since the completion of all five randomized clinical trials in 1992. We looked at the initial management with anticoagulation, antiplatelet agents, or neither anticoagulation nor anti-platelet agents.

Results: Of 262 patients over the age of 65 , only $3 \%$ were anticoagulated prior to 1992. This increased to $57 \%$ by 1995 . (chi square for trend, $\mathrm{p}<0.001$ ) In patients less than the age of 65 , where aspirin is acceptable therapy, only $13 \%$ were on aspirin or warfarin prior to 1990 . This increased to $95 \%$ by 1994/1995. (chi square for trend, $\mathrm{p}<0.001$ )

Conclusions: The trends at our hospital show that anticoagulation practices have certainly become more widespread since the conclusion of the randomized clinical trials in 1992. Although the trends are encouraging, much improvement is still necessary in the management of patients with NVAF.

\section{B. BRAIN TUMOURS}

\section{B-01}

The Assessment Of Primary Human Brain Tumor Cell Invasion In A New Three-Dimensional Invasion Assay

V.R. Amberger, W. McDonald, E. Moore, G. Bauman, B. Fisher, R.F. Del Maestro (London, Ontario)

Background: Failure to control malignant glial tumors after surgical removal, radiation and chemotherapy appears to be linked to an invasive subpopulation of malignant cells distant from the main tumor mass. We have developed a new assay to study cell invasion from human malignant brain tumor explants using a three-dimensional system to further elucidate the mechanisms underlying this invasive paradigm.

Methods: Human brain tumor explants removed at operation were implanted into collagen type I gels (Vitrogen 100) and cell invasion from the explants was monitored over $10-12$ days. The architectural components of the gel were altered by incorporating human CNS myelin homogenate. A number of gels were radiated and chemotherapeutic agents were also used to mimic the treatment that patients receive in vivo.

Results: Normal human white and grey matter showed no invasive activity in the model used. There was a direct relationship between the invasive activity of human tumor explants and the malignant grade of the original tumor. The addition of CNS myelin to the gel did not significantly alter the invasive activity of cells from glioblastoma whereas it decreased the invasion activity of meningioma and metastases explants.

Glioblastoma explant invasive activity appeared to have variable responses to radiation and chemotherapeutic agents suggesting that therapy may need to be individualized to obtain maximum efficacy.

Conclusions: The invasion of collagen type I three-dimensional gels by human brain tumor explants appears to be a good model to assess responses to treatment modalities used in the management of malignant cerebral tumors.

Supported by the Brain Tumor Foundation of Canada.

\section{B-02}

\section{Characterization of Human Brain Tumor Cell Cultures} Transduced with Proinflammatory Genes

I.F. Parney, A. Koshal, M. Cahill, M.A. Farr-Jones, L-J. Chang, K.C. Petruk (Edmonton, Alberta)

Immunogene therapy is a novel cancer treatment strategy based on vaccination with irradiated autologous tumor cells transduced with immunostimulatory genes. To characterize such cells prior to clinical applications, we studied a human glioma 
cell line (D54MG) and early passage human glioma (Ed147.BT, Ed149.BT) or melanoma (Ed141.MEL) cultures after therapeutic gene transfer.

Granulocyte-macrophage colony-stimulating factor (GMCSF), interleukin-12 (ILI2), and B7-2 genes were retrovirally transferred to tumor cells. Gene expression before and after radiation (20 $000 \mathrm{rad)}$ was assessed by ELISA (GM-CSF, IL12) and flow cytometry (B7-2). Wild type and gene-transduced tumor growth rates were determined by serial cell counts with a hemocytometer.

GM-CSF expression was high $(9.795-44.061$ pre- and 6.280 - $76.920 \mathrm{ng} / 106$ cells/day post-radiation) in GM-CSF-transduced culture. IL 12 expression was somewhat lower (1.300 14.660 pre- and $1.600-10.500 \mathrm{ng} / 106$ cells/day post-irradiation). B7-2 expression in B7-2-transduced cells was high (1 - 2 $\log$ increase in fluorescence) and unaffected by radiation. Growth rates were similar in wild type and gene-transduced Ed141.MEL, Ed147.BT, and Ed149.BT. However, IL12-transduced D54MG grew significantly slower than wild type.

GM-CSF, IL12, and B7-2 genes can be efficiently transferred to human glioma and melanoma cell cultures using our retroviral vectors. A radiation dose high enough to prevent further cell division does not significantly alter therapeutic gene expression. Growth rate is not altered by therapeutic gene expression in early passage cultures but was decreased by IL 12 in an immortalized cell line (D54MG). These results suggest that creating vaccines with autologous genetically-modified tumor cells is feasible.

\section{B-03}

\section{Expression of Growth Factor Receptors in Glioblastoma Multiforme Cell Lines and Tumor Specimens Results in Ras Activation and Ras-Dependent Tumor Proliferation}

Feldkamp, M.M., Lau, N., Guha, A. (Toronto, Ontario)

Glioblastoma multiforme (GBM) express high levels of growth factor receptors, particularly the epidermal and platelet derived growth factor receptors (EGF-R and PDGF-R). We have proposed that these receptors activate the Ras pathway in these cells, resulting in Ras-dependent proliferation.

EGF-R and PDGF-R status were evaluated in five established astrocytoma cell lines (U87, U118, U138, U343, and U373). Levels of activated Ras ${ }^{-G T P}$ were measured in these cell lines using a P-32 loading assay. Twenty operative GBM specimens were evaluated for levels of activated Ras ${ }^{\bullet} \mathrm{GTP}$ using a novel luciferase-based assay. The relevance of Ras activation in cell lines was evaluated using genetic (dominant negative Ras-N17) and pharmacological means (treatment with the farnesyl transferase inhibitor L-739,749). Activation of downstream mitogenic pathways (MAPK) was evaluated using a myelin basic protein (MBP) kinase assay.

The mean level of Ras ${ }^{\circ}$ GTP in the operative tumor specimens was $1.62 \pm 0.62$ femtomoles per $\mu \mathrm{g}$ of DNA, compared to levels of only 0.08 and $0.04 \mathrm{fmol} / \mu \mathrm{g}$ DNA in two non-neoplastic head injury specimens used as controls. Levels of Ras ${ }^{\circ}$ GTP in the five astrocytoma cell lines were similar to levels in $\mathrm{v}$-H-Rastransformed murine fibroblasts (RT8 cells), with approximately
$30 \%$ of Ras being in the activated (GTP-bound) form in these cells. Non-transformed human astrocytes also demonstrated lower levels of Ras.GTP $(11.2 \pm 1.1 \%$ of Ras in GTP-bound form). When Ras activity was inhibited using Ras-N17, proliferation and colony formation in soft agar were reduced to less than $50 \%$ of control cells, correlating with $50 \%$ reductions in Ras $\cdot$ GTP levels and $50 \%$ reductions in MAPK activity. Pharmacological Ras inhibition in U87 cells using the farnesyl transferase inhibitor (FTI) L-739,749 resulted in a $48 \%$ reduction in proliferation $(\mathrm{p}=0.0063$ by paired $\mathrm{t}$-test $)$ even at low doses $(10 \mu \mathrm{M})$ over short periods (12 days treatment).

These experiments confirm the relevance of Ras-mediated signaling in the molecular pathogenesis of GBMs. Activation of the Ras pathway is critical in the proliferation of these tumors, as evidenced by genetic manipulation (using Ras-NI7) as well as pharmacological inhibition (with L-739,749). While oncogenic mutations of Ras are not present in astrocytomas, the overexpression of surface receptors results in functionallyimportant activation of the Ras pathway, making such tumors potentially amenable to novel pharmacological agents which specifically target the Ras pathway.

\section{B-04}

\section{Photodynamic Therapy [Pdt] Of Supratentorial Gliomas}

Paul Muller, Brian Wilson (Toronto, Ontario)

We have treated 84 patients with supratentorial gliomas with PDT in a phase II setting. These patients received $2 \mathrm{mg} / \mathrm{kg}$ Photofin iv. 12-36 hours prior to surgical resection of their tumor or tumor cyst drainage.

There were 64 recurrent patients who had failed previous surgery and radiotherapy. The energy density range was $8-150$ $\mathrm{J} / \mathrm{cm}^{2}$ and the delivered light energy range was $440-7200 \mathrm{~J}$ (median=1700J). The median survival times in weeks for recurrent glioblastoma (37 patients), malignant astrocytoma ( 16 patients), malignant mixed astrocytoma-oligodendroglioma (7 patients) and ependymoma (4 patients) were $31,50,>64$ and $>261$ weeks, respectively. The thirteen patients with recurrent GBM who received $\geq 50 \mathrm{~J} / \mathrm{cm}^{2}$ had a median survival of 51 weeks. The eight patients with recurrent GBM who received $>60 \mathrm{~J} / \mathrm{cm}^{2}$ had a median survival of 58 weeks.

There were 20 patients with newly diagnosed malignant supratentorial gliomas. The energy density range was $15-110$ $\mathrm{J} / \mathrm{cm}^{2}\left[\right.$ median $\left.=32 \mathrm{~J} / \mathrm{cm}^{2}\right]$ and the delivered light energy range was $570-4050 \mathrm{~J}$ [median=1260J]. All but two had post-operative radiation therapy [5000 cGy in 5 weeks]. The median survival of 11 patients with newly diagnosed glioblastoma was 37 weeks with a 1 and 2 year survival of $35 \%$ and $0 \%$, respectively and the median survival for 9 patients with malignant astrocytoma was 48 weeks with a 1 and 2 year survival of $44 \%$ and $33 \%$, respectively. Six patients with a Karnofsky score of $>70$ who received a light dose of $>1260 \mathrm{~J}$ [mean energy density $=62$ $\pm 20 \mathrm{sem} \mathrm{J} / \mathrm{cm}^{2}$ ] had a median survival of 92 weeks with a 1 and 2 year survival of $83 \%$ and $33 \%$, respectively.

PDT is safe in patients with either newly diagnosed or recurrent supratentorial malignant gliomas. There appears to be 
prolongation of survival in selected patients when an adequate light dose is used. A randomized controlled phase III clinical trial of brain tumor PDT has been approved and funded by NIH-NCI.

\section{B-05}

The Alberta Neuroendoscopy Data Base: Preliminary Experience with 105 Patients

M. Hamilton, S.T. Myles (Calgary, Alberta), K. Aronyk (Edmonton, Alberta)

Diagnostic and interventional neuroendoscopy is rapidly becoming an important part of modern neurosurgery. The two neurosurgical centers in Alberta have initiated a data base to track the outcomes of patients who undergo a neuroendoscopic procedure. This review of the first 105 patients is based upon prospectively accumulated data for all patients who have undergone a neuroendoscopic procedure (excluding simple shunt placement) since initiation of this technique at each center.

Since 1994, a total of 105 patients have undergone 134 neuroendoscopic procedures during 111 separate surgical sessions. There were 58 male and 47 female patients. The age distribution included 6 newborns, 24 patients age 1 week to 2 years, 29 patients age 2 to 16 years, and 46 patients greater than 16 years. Primary diagnosis was aqueductal stenosis in 28 , arachnoid/ependymal cyst in 32 , brain tumor in 29 , colloid cyst in 5 , and other $(n=7)$. The type of procedure include 57 third ventriculostomies, 24 tumor biopsies or resection, 36 cyst fenestrations, 10 septostomies, 4 colloid cyst resections, and 3 others. Ten patients underwent successful removal of their VP shunt system and many of these procedures either eliminated the need for a shunt or the need for a second shunt. One hundred patients survive with a range of 1 month to 4 years. Five patients have died, 4 as a result of tumor progression and one from sudden death (nonhydrocephalus related). Complications included 3 IVH and 2 patients with a CSF leak. All 5 patients responded to standard care with no long term sequella. Thirteen patients failed primary treatment and required shunt insertion or replacement.

This type of ongoing data base is important to establish the indications and risks of neuroendoscopy. We hope to expand the data base to other parts of Canada and the United States.

\section{B-06}

\section{Unresectable Meningiomas: Role of Hydroxyurea Therapy}

F. Gentili, W. Mason, P. Gullane (Toronto, Ontario)

Despite the use of modern skull base approaches certain meningiomas arising from the skull base cannot be totally resected. We report on a series of 30 patients with unresectable meningiomas treated between 1982 and 1996. The majority of lesions involved extensive areas of the skull base, cavernous sinus, and petroclival region. The age and sex distribution was similar to those with lesions considered resectable. Histology revealed no frankly malignant lesions. Management strategies have included observation alone, surgery, hormonal manipulation and radiotherapy.
Ten patients with evidence of tumour progression on MRI received hydroxyurea $(20 \mathrm{mg} / \mathrm{kg} / \mathrm{day})$. All patients on hydroxyurea have stabilized clinically and radiologically (median follow-up 6 mos, range 3-7 months). No evidence of tumour regression has been observed. Hydroxyurea has been well tolerated. Only 3 patients required dose reduction for alleviation of myelosuppression. Severe toxicity has not been observed.

The average follow-up for the entire series has been 8 years (1-13 years). All patients are alive with residual disease. Functional status based on Karnofsky rating revealed $85 \%$ to be fully functional. $90 \%$ of patients have stable disease with no evidence of progression. In summary, a multi-modality approach including surgery, hormonal manipulation, radiation and chemotherapy with hydroxyurea can result in prolonged functional survival in this group of patients.

\section{B-07}

\section{"Malignant" Transformation Of Low Grade Astrocytomas}

PJ Muller, WS Tucker, R Moulton, M Cusimano, J Bilbao (Toronto, Ontario)

The "malignant" transformation of low grade astrocytic tumors [astrocytoma grade 2] to high grade astrocytic tumors [malignant astrocytoma or glioblastoma multiforme] is a well established phenomenon and has been shown to be an important feature in high grade tumor prognostication in that high grade tumors which have arisen from low grade tumors have a better prognosis that those high grade tumors diagnosed de novo.

However, much is to be learned about the rate and time course of these transformations from low to high grade tumors. We have analyzed a cohort of adults with low-grade astrocytomas. There were 95 males and 78 females; the mean age \pm S.D. of the group was $40.7 \pm 14.7$ years.

Of the 181 patients 48 [27\%] progressed under observation and came to reoperation. At the first reoperation 18/48 [38\%] had an astrocytoma grade 2, 14/48 [29\%] had an astrocytoma grade $3,11 / 48$ [23\%] had an astrocytoma grade 4 and 2/48 [4\%] had a mixed oligo-astocytoma. Thus, of those that came to the first reoperation $52 \%$ had a high grade tumor. The mean time to reoperation was $41 \pm 32$ months with a median of 37 months.

Of the 18 patients whose diagnosis at first reoperation was progression of low-grade astrocytoma, 7 went on to a second reoperation at which 6 of the 7 had either an MA or GB.

\section{B-09}

The Farnesyl Transferase Inhibitor L-744,832 Inhibits the Proliferation of Astrocytoma Cells, with Preferential Inhibition of Cells Expressing a Constitutively-activated Truncated EGF Receptor Common in Glioblastoma Multiforme

Feldkamp, M.M., Lau, N., Guha, A. (Toronto, Ontario)

Thirty percent of human cancers express oncogenic Ras mutations, resulting in constitutive activation of downstream 
Ras-dependent pathways. Oncogenic Ras mutations have not been identified in astrocytoma cell lines or tissues. However, we have previously demonstrated that the overexpression of growth factor receptors in these tumors results in functionally important Ras pathway activation. We sought to further investigate the functional role of Ras pathway activation in these tumors through pharmacological inhibition using the farnesyl transferase inhibitor (FTI) L-744,832.

Six astrocytoma cell lines were evaluated: U87, U118, U138, U343, U373, and U118:p140 EGF-R (expressing the common constitutively-activated truncated epidermal growth factor receptor pl $40^{\mathrm{EGF}-\mathrm{R}}$ ). Cells were treated with $0.01-100 \mu \mathrm{M} 744,832$. LD50 doses were estimated using non-linear logistic regression. Vascular endothelial growth factor (VEGF) secretion was measured using an ELISA assay.

All six cell lines were growth-inhibited by L-744,832 and were unaffected by vehicle alone. LD50 doses were estimated as follows: U138 $5.26 \mu \mathrm{M}, \mathrm{U} 373 \mathrm{I3.89} \mu \mathrm{M}, \mathrm{U} 8714.88 \mu \mathrm{M}$, U343 $17.38 \mu \mathrm{M}$, U118 11.21 $\mu \mathrm{M}$. U118:p140 EGF-R were growth inhibited at lower doses, with an LD50 of $4.36 \mu \mathrm{M}$, while U118 cells expressing the empty vector pcDNA3 had a LD50 similar to parental U118 cells. As the $95 \%$ confidence intervals for U118 and U118:p 140 ${ }^{\mathrm{EGF}-\mathrm{R}}$ cells do not overlap, these data demonstrate a statistically significant difference in drug sensitivity of cells expressing p $140^{\mathrm{EGF}-\mathrm{R}}$. L-744,832 reduced VEGF secretion by cells, even when the lower number of cells in drug-treated wells was accounted for. The effect of L-744,832 on VEGF secretion was most pronounced under hypoxic conditions.

While astrocytomas do not express oncogenic Ras mutations, overexpression of growth factor receptors results in functional Ras pathway upregulation. We have demonstrated that L744,832 inhibits proliferation as well as angiogenesis in astrocytoma cell lines, and offers hope that FTIs may hold promise in future clinical trials involving patients with GBMs. The subset of GBMs expressing the truncated receptor $\mathrm{pl} 40^{\mathrm{EGF}-\mathrm{R}}$ may be particularly sensitive to growth-inhibition by FTIs.

\section{B-10}

\section{Marked Inhibition of Tumor Growth in a Malignant Glioma Tumor Model by a Novel Synthetic Matrix Metalloproteinase Inhibitor AG3340}

A Price, NB Edwards, VW Yong, QS Zhong, P Brasher, D Edwards, R Johnson, D Morris, ME Wilcox, K Applet/D Shalinsky, P Forsyth (Calgary, Alberta)

Background: Matrix metalloproteinases (MMPs) have been implicated in the growth of gliomas by facilitating tumor invasion and angiogenesis. Synthetic MMP inhibitors have activity in a number of tumors in pre-clinical models but their usefulness in gliomas has never been demonstrated.

Objectives: To determine the effect of AG3340 on tumor growth of the human glioma cell line U87 in a murine model.

Methods: Tumors were grown in the left hind limb of SCIDnod mice and allowed to grow to a diameter of $0.5 \mathrm{~cm}$. when they were divided into two groups: 1) vehicle control (VC) and 2) AG3340. Both were injected with VC or AG3340 daily (100 $\mathrm{mg} / \mathrm{kg}$, intraperitoneal, Agouron Pharm.). Animals were sacrificed at a predetermined time and tumors, liver and lungs were excised.

Results: In vivo inhibition of tumor growth was profound and long lasting. Histological examination suggests AG3340 treated tumors have less proliferating cells and are less invasive.

Conclusions: These data suggest a profound inhibition of glioma tumor growth in this model. The mechanism of growth inhibition is unknown but may be partly mediated through blocking proliferation. Further studies to determine its mechanism of action, effectiveness in intracerebral glioma models and synergistic actions with radiotherapy are underway.

\section{B-11}

\section{False Negative Initial CT Scans in Patients with Malignant} Brain Tumors

\section{T. Jeerakathil, A. Shuaib, F. Kherani (Edmonton, Alberta)}

Background: Neurologists order plain head CTs in patients with headache and other symptoms to rule out serious intracranial pathology. With normal CT scan, the possibility of an intracranial cause is dismissed with reasonable certainty. Often, no follow-up scans are performed.

Methods: Three cases are presented in which initial CT scans revealed no apparent cause for the patients' symptoms. Imaging performed within months of the initial scans revealed malignant brain tumors.

Results: A 54-year-old female experienced severe, intermittent, headaches starting in early 1997.

A CT head performed in May 1997 was normal. In October she developed nausea, confusion, and unsteadiness. She was found to have a left hemiparesis, left hemisensory loss. CT head revealed a large right temporoparietal mass lesion with vasogenic edema and subfalcine herniation. Subsequent CT head with contrist revealed irregular ring enhancement. Brain biopsy revealed glioblastoma multiforme (GBM). A 52-year-old male presented with intermittent confusion. Neurological exam revealed speech arrest but little else. Initial CT scan and a subsequent scan were normal. MRI scan two months later revealed multiple small lesions thought to be infarcts. Patient was anticoagulated but continued to have symptoms. Repeat MRI months later revealed mass lesion with multiple areas of ring enhancement. Brain biopsy revealed GBM. A 66year-old female presented with confusion. She was admitted to a medical ward and found to have a serum sodium of 125 , and a potassium of 2.3. Her electrolyte abnormalities were corrected. CT head was normal and her confusion improved. Over the next two months she developed mild confusion, falls, unsteadiness, and speech difficulty. Over a period of two days she developed left facial droop, left hemiparesis, and generalized seizures. CT head revealed a right frontotemporal hypodensity with mass effect. vasogenic edema and subfalcine herniation. Enhanced CT scan revealed ring enhancement likely a GBM.

Conclusions: These case presentations suggest that a single CT scan is not reliable in ruling out serious intracranial pathology in all cases. Serial imaging may be required, especially with continued symptoms, and definitely with any clinical worsening. 


\section{EPILEPSY}

\section{$\mathrm{C01}$}

\section{Comparison of Dynorphin and Calbindin Immunoreactivity in Mesial Temporal Sclerosis}

PV Gould, R Desbiens, F Cicchetti, A Parent (Québec, Québec)

One of the characteristic changes described in temporal lobe epilepsy is the sprouting of mossy fibers which project from the dentate granule cells onto hilar interneurons and CA3 pyramidal cells. We have examined a series of twelve hippocampal resections from patients with mesial temporal sclerosis (MTS) who underwent surgery for intractable epilepsy in the period 1996-1997. The degree of mossy fiber sprouting was assessed using two different immunohistochemical markers, the calcium binding protein calbindin-28K (CB) and the peptide dynorphin A (dynA).

As expected both markers were positive in dentate granule cells and their projections: however a greater number of mossy fiber projections showed dynA immunoreactivity. Occasional hilar interneurons and dentate granule molecular layer neurons were immunoreactive for $C B$ in three cases but only one case showed dynA immunoreactivity in hilar interneurons. This dynA immunoreactivity occurred in a microscopic neuronal heterotopia and was never observed in autopsy controls. In four cases with near total loss of hilar interneurons there was a redistribution of dynA immunoreactivity with increased staining of the dentate granule molecular layer in comparison to cases with partial preservation of hilar interneurons. Although CB immunoreactivity was generally weaker in the cases with loss of hilar interneurons, the distribution of staining remained similar to that in less severely affected cases. Evaluation of a larger series is in progress to determine whether these patterns of immunoreactivity can be correlated with the clinical evolution of these patients.

\section{C-02}

\section{Peripheral Visual Field Constriction in Patients on Vigabatrin}

\section{A.Guberman, R. Mclachlan, D. Zackon (Ottawa, Ontario)}

Vigabatrin, a GABA-transaminase inhibitor which elevates brain and retinal GABA, is widely used for partial epilepsy and infantile spasms. Concentric visual field constriction has been reported recently in 12 European patients. The mechanism of retinal toxicity, its incidence, specificity for vigabatrin and reversibility are unknown. We have studied 6 cases of bilateral visual field constriction on vigabatrin at 2 Canadian centres.

There were 5 males and 1 female aged 33-55. Mean duration on vigabatrin was 49.5 months (18 months-6.5 years) and mean daily dose was $3.1 \mathrm{~g}$ (1.5-4 g). Three patients were asymptomatic , 3 noted peripheral visual loss , 4 had abnormal confrontation testing . Visual acuity was unaffected; mild optic disc pallor occurred in 3 cases. All patients showed significant concentric visual field constriction on static (Humphrey) perimetry. Two patients showed progression of field defects on the drug.

This type of visual field constriction is very unusual in any other situation aside from long-term vigabatrin treatment. Patients should be warned to report visual symptoms, confrontation fields should be done in asymptomatic patients every 6 months, formal static fields should be done in symptomatic patients every 3 months. The decision to continue vigabatrin in affected patients should be made according to risk/benefit considerations.

\section{C-03}

\section{Piracetam in the Treatment of Cortical Myoclonus}

M Fedi, D D'Agostino, F Dubeau, D Reutens, E Andermann, F Andermann (Montreal, Quebec)

Background: In patients with myoclonic epilepsy the myoclonus is often very disabling and difficult to treat. It has been suggested that piracetam is an effective antimyoclonic agent. The aim of this study is to verify the clinical effect of piracetam in patients with intractable myoclonus.

Methods and Results: We retrospectively studied nine patients ( 6 men, 3 women) with cortical myoclonus due to progressive myoclonic epilepsy in eight and Lance Adams syndrome in one. Most had severe, highly disabling action and spontaneous myoclonus with inadequate pharmacological control. The degree of disability and the frequency of myoclonus were evaluated by reviewing the medical records, examination and analysing the videos when available. The total daily dose of piracetam, given in 2 or 3 doses, ranged from 50 to $300 \mathrm{mg} / \mathrm{kg}$. We compared the data six months before and after beginning treatment with piracetam. Marked reduction of myoclonus was observed in four patients, moderate reduction in four, and slight reduction in one. None complained of side effects.

Conclusion: Polytherapy with piracetam in combination with clonazepam and valproate is an effective symptomatic treatment of cortical myoclonus. Further trials in postantoxic myoclonus and in various forms of epileptic myoclonus are indicated.

\section{C-04}

Autosomal Dominant Partial Epilepsy with Variable Foci: A New Syndrome in French Canadians

E Andermann, L Xiong, M Labuda, M Pandolfo, F Dubeau, F Andermann (Montreal, Quebec), S Berkovic (Melbourne, Australia), R Desbiens, S Verret, G Patry, P Langevin (Quebec City, Quebec)

Background: In recent years, a number of single-gene epilepsy syndromes have been described, including four new autosomal dominant partial epilepsy syndromes. We describe two large French Canadian families presenting with a novel form of partial epilepsy with variable foci.

Method: The probands were ascertained at the Montreal Neurological Hospital and initially diagnosed to have familial frontal lobe epilepsy. The family members were investigated 
during three field trips to the Quebec City region. Detailed epilepsy histories, neurological examinations, medical records, and blood samples for genetic studies, were obtained on all available family members. Routine and sleep EEG examinations on family members are being carried out.

Results: Over 30 affected individuals were ascertained in the two families. They had either frontal or temporal lobe epilepsy, mainly nocturnal but frequently diurnal. Many family members reported a history of nightmares, sleepwalking and déja vu. The two families originated from the same small town northwest of Quebec City and had common surnames, suggesting founder effect. Preliminary linkage analysis appears to exclude candidate loci on chromosomes $20 \mathrm{q}, 15 \mathrm{q}, 10 \mathrm{q}, 8 \mathrm{q}$, and $19 \mathrm{q}$.

Conclusion: A new autosomal dominant partial epilepsy syndrome is described in two French Canadian kindreds with possible founder effect. Genetic studies have excluded a number of known loci for epilepsy genes. A genome screen employing fluorescent based automatic technology is underway.

\section{C-05}

\section{Clinical and Genetic Studies in Familial Temporal Lobe Epilepsy}

M.D. D'Agostino, E. Andermann, M. Labuda, M. Pandolfo, F. Andermann, I. Lopes-Cendes, F. Cendes (Montreal, Quebec), S.F. Berkovic (Melbourne, Australia), B. Abou Khalil (Nashville,Tennesse), M. Javidan (Edmonton, Alberta)

Background: Most partial epilepsies represent complex phenotypes with polygenic inheritance. Recently a group of idiopathic partial epilepsies with single gene inheritance has been identified, including familial temporal lobe epilepsy ( FTLE). Several candidate loci for linkage studies in FTLE have been identified. Our aims are to identify families with FTLE, to define the clinical spectrum of the syndrome, and to clarify the genetic basis of FTLE.

Methods: Families where two or more members had TLE have been ascertained from five centers in three continents. Previous medical records, EEGs and MRI scans of affected individuals were reviewed and repeated if necessary. Clinical histories, EEGs and blood samples for DNA studies were obtained from relatives whenever possible. Preliminary genetic linkage studies for known epileptic loci have been initiated.

Results: We identified 25 families with FTLE. In 11 families, individuals were seen in more than one generation. 18 families had benign FTLE with an overrepresentation of deja vu and migraine. While most patients with FTLE show a benign course, others require surgical intervention, indicating possible genetic heterogeneity. In one large family, preliminary genetic studies suggested linkage to markers on chromosome 10q.

Conclusions: FTLE is not a rare disorder, and may have a heterogeneous clinical course. Pedigree analysis in our families suggests autosomal dominant inheritance with reduced penetrance.

\section{Clinical, Genetic and Neuroimaging Features in Three Male Patients with Subcortical Band Heterotopia}

M.D.D'Agostino, E. Andermann, F. Andermann, F. Dubeau, M. Fedi, A. Bastos, (Montreal, Quebec); F. Booth (Winnipeg, Manitoba)

Background: Subcortical band heterotopia ( $\mathrm{SBH})$ is a neuronal migration disorder, usually sporadic, that occurs predominantly in females. We report three males with SBH to highlight the relationship between the extent of the band and the severity of the seizures these patients present, and to clarify the genetic basis of SBH.

Methods: The patients were ascertained in our center. Detailed information regarding family history of epilepsy, abnormal prenatal events, psychomotor development, and age of onset of seizures was available. Patients underwent neurologic examination, neuropsychologic testing and prolonged videoEEG monitoring. In addition to high resolution MRI studies, three dimensional surface rendering was obtained using curvilinear reconstruction of the MRIs: images were displayed at 6 $\mathrm{mm}$ from the surface. Chromosome studies, fluorescence in situ hybridization (FISH) analysis of the lissencephaly region on chromosome 17p13.3, and mutation analysis of the XLIS gene were performed in each patient.

Result: Patient $I$ had a thin band confined to the parietaloccipital regions; he had complex partial seizures well controlled by medications, normal neurological examination and intelligence. Patient 2 had an intermediate and diffuse band, mild pachygyria, enlarged ventricles, and Lennox-Gastaut syndrome (LGs) with severe cognitive dysfunction. He underwent callosotomy. Patient 3 had a diffuse and thick band, agyria, enlarged ventricles, thin corpus callosum and atrophic cerebellum. He had had West syndrome, evolved into LGs, had several dysmorphic features, and was profoundly mentally retarded. The three patients had normal male karyotypes. FISH analysis results are pending.

Conclusions: There is a direct correlation between the extent of the band and the severity of the epileptic syndrome these patients present. Further genetic investigations are needed to clarify the etiology of the disease.

\section{C-07}

\section{Complications in Functional Hemispherectomy: Observations in 63 Patients}

JG Villemure (Lausanne, Switzerland), JP Farmer, C Mercier, A Turmel, JL Montes (Montreal, Quebec)

Functional hemispherectomy consists of anatomical subtotal removal of the hemisphere but complete disconnection. We report and discuss the complications observed in 63 cases of functional hemispherectomy for control of seizures, at a median follow-up of 7 years.

Three deaths were related to the operations. The causes were: 1) severe oedema of the disconnected hemisphere on the third post-operative day, 2) a hemorragic infarct involving brainstem structures, 3) a cardiac arrest occurring 6 hours after an uneventfull recovery from surgery; in this latter case, the autopsy did 
not confirm the exact cause of death. One patient developed an abcess in the disconnected hemisphere, which was successfully treated medically. Four (4) patients developed early post-operative hydrocephalus requiring a CSF shunt. One patient required reoperation to extend posteriorly the basal frontal disconnection. Seizure control is as follows: $77 \%$ seizure-free, $17 \%$ improved by at least $80 \%$ and $6 \%$ unchanged.

Functional hemispherectomy is an effective method of hemispherectomy for control of refractory seizures. Since it provides the same results toward seizure control, but has the lowest complication rate (from published data) as compared to other methods of hemispherectomy, it appears to be the technique of choice.

\section{C.08}

Localization of Supplementary Motor Area by fMRI Using a Complex Motor Task: Validation with Intraoperative Stimulation

J.D. Atkinson, M. Cyr, R. Hoge, G.B. Pike, A.F. Sadikot (Montreal, Quebec)

Background: The supplementary motor area (SMA) is known to be involved in the preparatory phase of complex movements. Motor tasks that are complex and involve temporal or spatial decision making should, thus, produce activation patterns on functional imaging studies. We present here a reliable method for SMA activation by functional magnetic resonance imaging (fMRI) which has been validated with intraoperative stimulation.

Methods: A complex motor task consisting of self selected random joystick movements paced by a $1 \mathrm{~Hz}$ auditory stimulus was applied to a group of healthy volunteers $(n=6)$ and one patient with a right frontal tumour. fMRI studies were performed using a Siemens Vision 1.5T scanner and an echo planar imaging (EPI) sequence producing blood oxygen level dependent (BOLD) contrast. Patient data were taken to the operating room and compared with frameless stereotaxy based stimulation localization.

Results: The random direction self selection task produced clearly increased SMA activation in all subjects in comparision with a unidirectional task. Activation of the left SMA only was seen in the patient with the right frontal lesion despite testing the left hand. Intraoperative stimulation of both primary motor areas and SMA produced excellent concordance with the FMRI results.

Conclusions: SMA activation can be reliably produced using a complex motor task such as the one described here. SMA and primary motor cortex localization using fMRI corresponds well to the results of intraoperative stimulation.

\section{C-10}

Clinical, Genetic and Neuroimaging features in Three Male Patients with Subcortical Band Heterotopia

MD D'Agostino, E Andermann, F Andermann, F Dubeau, M Fedi, A Bastos (Montreal, Quebec); F Booth (Winnipeg, Manitoba)

Background: Subcortical band heterotopia (SBH) is a neuronal migration disorder, usually sporadic, that occurs predomi- nantly in females. We report three males with SBH to highlight the relationship between the extent of the band and the severity of the seizures these patients present, and to clarify the genetic basis of SBH.

Methods: The patients were ascertained in our center. Detailed information regarding family history of epilepsy, abnormal prenatal events, psychomotor development, and age of onset of seizures was available. Patients underwent neurologic examination, neuropsychologic testing and prolonged videoEEG monitoring. In addition to high resolution MRI studies, three dimentional surface rendering was obtained using curvilinear reconstruction of the MRIs: images were displayed at $6 \mathrm{~mm}$ from the surface. Chromosome studies, fluorescence in situ hybridization (FISH) analysis of the lissencephaly region on chromosom 17p13.3, and mutation analysis of the XLIS gene were performed in each patient.

Result: Patient $I$ had a thin band confined to the parietaloccipital regions; he had complex partial seizures well controlled by medications, normal neurological examination and intelligence. Patient 2 had an intermediate and diffuse band, mild pachygyra, enlarged ventricles, and Lennox-Gastaut syndrome (LGs) with severe cognitive dysfunction. He underwent callosotomy. Patient 3 had a diffuse and thick band, agyria, enlarged ventricles, thin corpus callosum and atrophic cerebellum. He had West syndrome, evolved into LGs, had several dysmorphic features, and was profoundly mentally retarded. The three patients had normal male karyotypes. FISH analysis results are pending.

Conclusions: There is a direct correlation between the extent of the band and the severity of the epileptic syndrome these patients present. Further genetic investigations are needed to clarify the etiology of the disease.

\section{C-11}

\section{Mesial Temporal Sclerosis in Hemifacial Atrophy}

M Sundaram, A Bowles, J Fratkin, J Corbett, A Leis (Jackson, Mississippi) K Digre (Salt Lake City, Utah)

Background: Mesial temporal sclerosis (MTS) is a common finding in temporal lobe epilepsy. Pathogenesis of MTS is controversial but childhood febrile seizures are thought to trigger progressive hippocampal changes which eventually result in chronic epilepsy. We, for the first time, describe 2 patients with hemifacial atrophy and MTS.

Case Reports: Mr. D C, 47 years old, developed progressive atrophy of the right side of the face during childhood. Complex partial seizures started when he was 22 and have remained intractable in spite of various anticonvulsants. He denied any febrile seizures. Telemetry confirmed right mid/anterior temporal focus. MRI showed right amygdala and hippocampal atrophy. He underwent surgery and pathology will be presented.

Miss C M, 28 years old, developed seizures at the age of 24 without previous febrile seizures. Examination showed facial atrophy on the right and ictal EEG confirmed right temporal focus. Neuroimaging revealed right mesial temporal atrophy and signal abnormalities in the right mesial frontal as well as mesial temporal and parahippocampal gyrus. Seizures still remain intractable. 
Conclusions: MTS may be responsible for seizures in hemifacial atrophy and may also precede seizures in some of these patients. This association suggests that the pathogenesis of MTS may be more heterogenous than currently held.

\section{SPINAL CORD}

\section{D-01}

\section{The Epidemiology Of Spinal Injuries From A Regional Trauma Unit}

\section{V.S.S.V. Prasad, M.L. Schwartz (Toronto, Ontario)}

Background: The epidemiological profile of spinal injuries is known to vary with time, place and origin of data. The objective of this presentation was to analyze the demographics of spinal injury in a multitrauma population and to identify the predisposing factors of severe spinal cord lesions.

Methods: The computerized data base (1987-96), collected prospectively, was reviewed and compared to the data provided by the Ontario Trauma Registry (OTR), wherever applicable.

Results: Spinal injuries (SI) were identified in 1197 patients accounting for $24 \%$ of the trauma population at the regional trauma unit. Cervical spine was the commonest level injured followed by the thoracic spine. Young men predominated $(95 \%$ industrial and motor cycle crash(MCC), $87 \%$ of recreational injuries). In females, the lumbar spine was the commonest level. At the cervical spine $\mathrm{C} 2$ was the commonest level $(28.5 \%)$; mostly with SI(73\%), followed by C5 (22\%) with SCI in $50 \%$. The majority of the young had thoracolumbar SI (70\% were $<40$ $\mathrm{yr}$ ). With increasing age, the pedestrian injuries, CSI(especially at $\mathrm{C} 2, \mathrm{Cl} ; \mathrm{p}=0.04)$ and female gender were often noted. Motor vehicle crash (MVC) was the commonest cause, followed by falls and pedestrian trauma. The majority of $\mathrm{SCl}$ involved the cervical spine; $\mathrm{MVC}$ (driver) was responsible for $44 \%$ of these and $28 \%$ at the thoracic spine. At the lumbar spine falls and MVC(rear passenger), $24 \%$ each resulted in SCI. $29 \%$ of MCC, $26 \%$ of recreational injuries and $24 \%$ of $\mathrm{MVC}$ (rear passenger) presented with SCl; but only $18 \%$ of $\mathrm{MVC}$ (driver) had SCI. In contrast to the OTR data, where $43 \%$ of SCI was caused by falls and $39 \%$ by MVC, our multitrauma population had SCI due to MVC and falls in $57 \%$ and $12 \%$ respectively $(\mathrm{p}<0.0001)$.

Conclusions: Complete SCI was more frequent in the young, compared to those $>50 \mathrm{yr}(\mathrm{p}=0.03)$, at the cervical spine than lumbar spine $(\mathrm{p}=0.008)$, at $\mathrm{C} 5+\mathrm{C} 4$ compared to $\mathrm{Cl}+2$ $(p<0.00001)$ and caused by MCC more than MVC $(p=0.03)$.

\section{D-02}

The Optimal Radiological Method to Assess Spinal Canal Compromise and Cord Compression in Patients with Cervical Spinal Cord Injury: Part 2: A Multi-center Cooperative Study

M Fehlings, SC Rao, C Tator, G Skaf (Toronto, Ontario)

In anticipation of a prospective, randomized trial of decom- pression in cervical spinal cord injury (STASCIS), we conducted a multi-center, retrospective study of CT and MRI assessments of canal compromise and cord compression. The study included 71 patients $(55 \mathrm{M}, 16 \mathrm{~F}$; mean age $39.7+18.7$ years) with acute cervical cord injuries (SCI) who were examined by $\mathrm{CT}$ and T1/T2 weighted MRI. Causes of SCI included motor vehicle accidents $(n=36)$, falls $(n=20)$, water-related injuries $(n=8)$, sports $(n=5)$, assault $(n=1)$ and farm accident $(n=1)$. There was good agreement between two independent observers for all observations $(R=0.763$ to $0.972 ; p<1 \times 10-10)$. There was a strong correlation of canal compromise or cord compression measurements between axial and mid-sagittal $C T(R=0.720$, $\mathrm{p}=0.000344$ ), and between axial and mid-sagittal T2 MRIs $(R=0.806, p=0.0000104)$. Cord compression was significantly greater when assessed by T2-weighted MRI than when assessed by $\mathrm{Tl}$-weighted MRI $(p=0.02)$. Spinal canal compromise by CT showed a significant correlation with spinal cord compression as assessed by both $\mathrm{Tl}(\mathrm{R}=0.610, \mathrm{p}=1.69 \times 10-8)$ and $\mathrm{T} 2$-weighted MRI $(R=0.511, p=5.36 \times 10-6)$. In conclusion, in patients with cervical SCl, the mid-sagittal T1 and T2-weighted MRI provides an objective, quantifiable and reliable assessment of spinal cord compression which cannot be adequately assessed by CT alone.

\section{D-03}

Post-Traumatic Syringomyelia: Predisposing Factors And Prognostic Indicators

V.S.S.V. Prasad, D.W.Rowed, N.Bharatwal (North York, Ontario)

Background: Post-traumatic cavitation of the spinal cord has been recognized for over a century and progressive ascending myelopathy associated with post-traumatic syrinx(PTS) for over 30 years. Prognostic indicators for development of PTS have not been well defined.

Methods: 47 consecutive PTS patients treated at the University of Toronto neurosurgical units between 1980 and 1997 were analyzed retrospectively to discover prognostic indicators for the development of PTS and to assess outcome.

Results: There were 36 males and 11 females with a mean age of 40.7 years. 27 patients had cervical spinal cord injury (CSCI) and 20 had thoracolumbar injuries(TLSCI). Neurological deficit was complete in $33 / 47$ PTS patients (70\%) compared with $47 \%$ of concurrent spinal cord injuries in general. Patients with complete injuries were therefore significantly more likely to develop PTS $(P=0.006)$. Onset was earlier with CSCI $(P=0.09)$, with complete neurological deficit, with fracture dislocations $(\mathrm{P}=0.04)$ and with younger age $(\mathrm{P}=0.007)$ and in cases where spinal fusion was performed $(\mathrm{P}=0.001)$. 16/23 patients $(70 \%)$ who were subjected to syringosubarachnoid shunts (SSS) improved compared with $7 / 14(50 \%)$ managed by syringopleural (SPIS) or syringoperitoneal (SPS) shunts. This may represent a trend in favour of improved outcome with SSS $(\mathrm{P}=0.3)$. Following shunting 14/20 TLSCI improved compared with 9/27 CSCI ( $P=0.02)$. Ordinal logistic regression modelling failed to provide any independent covariate relationship with outcome. 
Conclusions: PTS occurs more frequently following complete SCI. The onset is earlier with complete injuries, with $\mathrm{CSCI}$, with fracture dislocation, with younger age, and with spinal fusion. Overall results of management were good: $77 \%$ improved or remained neurologically stable. PTS associated with TLSCI had better outcome following shunting than did CSCI. Shunts are effective in managing PTS with a possible trend to better results with SSS.

\section{D-04}

\section{Motoneuron Survival and Axon Regeneration Following Insertion of a Peripheral Nerve Graft into the Spinal Cord of Adult Rats with C5 \& C6 Root Avulsion}

\section{T.J. Zwimpfer, C. Tarazi, J. Liu (Vancouver, British Columbia)}

It has been demonstrated in animals, that following spinal nerve root avulsion, injured motoneurons (MNs) can extend axons into ventral roots or peripheral nerve (PN) grafts immediately inserted into the spinal cord, with functional re-innervation of denervated muscles. Delayed re-implantation in human cases of root avulsion has been reported but the effect of such a delay on axon regeneration has not been studied in animals.

Following avulsion of the left C5 \& C6 ventral and dorsal roots in adult rats, one end of a $3.5 \mathrm{~cm}$ segment of common peroneal nerve was inserted 1 to $2 \mathrm{~mm}$ into the ventrolateral aspect of the spinal cord at C5 \& C6. Animals were divided into five groups based on the interval between root avulsion and PN graft insertion: Immediate, 1 week or 1, 2 and 6 months. Two months after insertion, a fluorescent axonal tracer, Rhodamine-Dextran amine (RDA) was applied to the free end of the graft. RDAlabeled neurons were present in 43 of 44 animals examined, the majority in the ipsilateral ventral horn. Using counts of RDAlabeled neurons in the immediate insertion group ( $\mathrm{n}=11$ animals; mean of 761 neurons $=100 \%$ ) as a reference for comparison, the number of RDA-labeled neurons in the 1 week group was $82 \%(\mathrm{n}=10$ animals), $44 \%$ at 1 month $(n=9)$ and $38 \%$ at both 2 months $(n=9)$ and 6 months $(n=5)$.

To allow identification of $\mathrm{C} 5$ \& $\mathrm{C} 6 \mathrm{MNs}$, additional studies were carried out in which fluorogold $(\mathrm{FG})$ was injected into the C5 \& C6 spinal nerves several days prior to root avulsion followed by PN graft insertion into the cord either immediately, 10 days or 1 month after root avulsion. RDA was applied to the end of the graft 2 months later. Based on counts of FG, RDA or double-labeled neurons, a 1 month delay in PN graft insertion resulted in a statistically significant decrease in: 1) the number of surviving C5 \& C6 MNs and 2) the proportion of surviving C5 \& C6 MNs that innervated the PN graft. These results indicate that implantation of PN grafts in human root avulsion injuries would need to be done in the first few weeks after injury. In addition, strategies to improve the extent of $\mathrm{MN}$ axon regeneration into PN grafts implanted into the spinal cord after root avulsion should focus on both improving survival of injured $\mathrm{MNs}$ and promoting axon regeneration of surviving MNs. Continuous intrathecal infusion of BDNF or GDNF is currently being studied in this rat model.

\section{D-05}

Vascular Endothelial Growth Factor (VEGF) Expression and Angiogenesis in Peripheral Nerve Lesions

Lilyana Angelov, Ian MacKenzie, Abhijit Guha (Toronto, Ontario)

To our knowledge, no study exists where the vascularity of peripheral nerve lesions and its relation to growth potential has been determined in a systematic fashion. This study evaluates the vascularity and expression of vascular endothelial growth factor (VEGF) one of the most important inducers of angiogenesis, vital for the continued growth of many tumours, across a spectrum of NF-1 and non-NF-1 peripheral nerve lesions.

37 specimens were evaluated from the following subgroups: i) normal nerve, ii) traumatic neuroma, iii) dermal-NF-1 neurofibroma, iv) plexiform-NF-1 neurofibroma, v) plexiform-nonNF-1 neurofibroma, vi) schwannoma, vii) NF-1 neurogenic sarcomas and viii) non-NF-1 neurogenic sarcoma. Vascularity was determined by immunohistochemistry (anti-Factor VIII antibody), and counts derived by averaging the number of vessels/four high powered scan areas (V/HPS) using computerized image analysis. IHC for VEGF protein was determined on adjacent sections and expressed as the proportion of positive staining/total scanned area .

In the study, normal nerves had 14 V/HPS, benign peripheral nerve lesions had between 17-35 V/HPS, with a slightly greater vascularity in traumatic neurofibromas. In contrast, the neurogenic sarcomas were highly vascularized with $90-131$ V/HPS. VEGF positivity paralleled the vascularity: normal nerves 3\%; benign lesions 6-17\%; while neurogenic sarcomas (NF-1 and non-NF-1) ranged between $43-50 \%$ resulting in a correlation of $0.82(\mathrm{p}<.0001)$ in these specimens.

Hence, increased vascularity is related to the proliferative capacity of peripheral nerve lesions (i.e. normal nerve<benign tumours<neurogenic sarcomas) and this difference in vascularity correlates to VEGF expression ( $>>0001$ ). Future strategies include modulation of VEGF expression and activity in these human peripheral nerve tumours with potential therapeutic implications.

\section{D-06}

Role of Group I Metabotropic Glutamate Receptors in Traumatic Spinal Cord White Matter Injury

MG Fehlings, S. Agrawal, E. Theriault (Toronto, Ontario)

Metabotropic glutamate receptors (mGluRs) participate in glutamate neural transmission but their role in the pathophysiology of spinal cord injury (SCI) has not been explored. Accordingly, we examined the role of Group I mGluRs in mediating SCI using an in vitro model. A dorsal column segment was isolated from the spinal cord of adult rats, maintained in vitro, and injured by compression for $15 \mathrm{sec}$ with a clip having a $2 \mathrm{~g}$ closing force. Under control conditions after $\mathrm{SCI}$, the compound action potential (CAP) amplitude was reduced to $69.1 \pm 5.4 \%$ of baseline. Blockade of Group I mGluR receptors with MCPG 
or $4 \mathrm{CPG}$ resulted in improved recovery of CAP amplitude. The Group I agonist DHPG and Group I/II agonist trans-ACPD exacerbated the post-traumatic reduction of CAP amplitude. The phospholipase C inhibitor U-73122 improved recovery of CAP amplitude after SCI and also blocked the deleterious effects of DHPG on post-traumatic recovery of CAP amplitude. Western blotting and immunocytochemistry demonstrated the presence of mGluR $\mid \alpha$-immunopositive periaxonal astrocytes and the absence of mGluR5 in spinal cord white matter.

In summary, these studies demonstrate that activation of Group I mGluR receptors after SCI exacerbates posttraumatic axonal injury through a phospholipase $\mathrm{C}$ dependent mechanism. The presence of mGluR $1 \alpha$ labelling on periaxonal astrocytes suggests a role for these cells in the pathophysiology of SCI.

\section{E. GENERAL NEUROSURGERY}

\section{E-01}

\section{Microvascular Decompression for Hemifacial Spasm}

AM Kaufmann (Calgary, Alberta)

The only potentially definitive treatment for hemifacial spasm (HFS) is microvascular decompression (MVD). The efficacy and safety of this procedure has evolved over 30 years, since first described by Gardner and subsequently refined by Jannetta.

A series of over $110 \mathrm{MVD}$ procedures were performed by the author between July 1996 and December 1997 at two university centers. This included 13 patients with HFS, aged 23 to 68 years. The duration of typical HFS was 7 months to 18 years (mean 5 years). One third had received multiple botulinum toxin injections pre-operatively.

Following MVD, 11 of 13 patients had no further HFS (85\% complete response at 3 to 16 months follow up). One 23-yearold woman with an 18-year history of HFS had occasional mild spasms at 1-year follow up, and was satisfied with the results. Another patient with an 8 year history of severe HFS and repeated botulinum toxin injections had an inadequate MVD response at 4 months and was scheduled for re-exploration. No serious morbidity occurred in the series. One post-operative CSF leak was treated with 4 days of lumbar drainage. No patient developed new deafness, although one woman with tic convulsif had further decline of pre-existing hearing loss following MVD.

MVD is a relatively safe and very effective treatment of HFS, and should be considered early in the course of this progressive and potentially disabling disease.

\section{E-02}

\section{A Neuroanatomical Data Guide. A New Surgical Approach for the Caudalis DREZ Operation}

M. Abdulhak (London, Ontario), A. El-Nagger, J. OvelmenLevitt, B.S. Nashold, Jr. (Durham, North Carolina)

Background: Anaesthesia dolorosa and post-herpetic pain of the face are significantly improved after the caudalis DREZ operation which is based on the thermal destruction of the nucleus at the cervicomedullary junction using a special electrode. It has been important to know exact dimensions and relationships of adjacent nuclear groups as well as fiber tracts in the cervicomedullary junction so that these lesions can be effective without compromising additional sensory and motor functions. Among important structures adjacent to the nucleus caudalis at different levels are the pyramidal tract, the spinocerebellar tracts, the spinothalamic tract and the dorsal columns.

Methods/Results: The neuroanatomy of the caudalis nucleus and its relationships were studied at multiple levels throughout 25 $\mathrm{mm}$ from $5 \mathrm{~mm}$ above to $20 \mathrm{~mm}$ below the obex. Fresh, followed by fixed, and then finally the histologically prepared sections of the same normal human brainstems were studied and measured. The marginal cell layer (lamina I) was $.5-1.0 \mathrm{~mm}$ from the dorsolateral surface of the spinal cord at $\mathrm{C} 2$ and $\mathrm{Cl}$; while at the obex and $5 \mathrm{~mm}$ rostral to the obex, these dimensions were $2.0-2.8 \mathrm{~mm}$ and $2.5-3.0 \mathrm{~mm}$, respectively. The nucleus was $1.5 \mathrm{~mm}$ in diameter at $\mathrm{C} 2,2.0 \mathrm{~mm}$ at $\mathrm{Cl}, 2.5 \mathrm{~mm}$ at the obex, while $5 \mathrm{~mm}$ above the obex it was $2.0 \mathrm{~mm}$. Adjacent structures which may be at risk during lesion production were found to be the pyramidal tract at $\mathrm{Cl}$ and $\mathrm{C} 2$, the afferents to and the external cuneate nucleus at and above the obex, the rubrospinal tract at all levels, the spinothalamic and spinocerebellar tracts at and above the obex. New electrode design and lesion parameters will be facilitated by this study.

Conclusion: The size of the nucleus caudalis changes both in configuration and in size depending in the rostrocaudal level. The proximity of the nucleus caudalis to the medulla surface also varies. The results of this anatomical study have provided a basis for the construction of two new electrodes each designed to be used at a certain position along the rostrocaudal length of the nucleus caudalis.

\section{E-03}

\section{A Biomechanical Evaluation of Occipito-Cervical Instrumentation: Screw versus Wire Fixation}

R. John Hurlbert, Neil R. Crawford, Won G. Choi, Curtis A. Dickman (Calgary, Alberta)

Objectives: The purpose of this study was to compare cable techniques of occipito-cervical fixation to two types of screw fixation. We hypothesized that screw fixation would provide superior immobilization compared to cable methods.

Methods: Ten cadaveric specimens were prepared for biomechanical analyses using standard techniques. Angular and linear displacement data were recorded from the occiput to $\mathrm{C} 6$ with infra-red optical sensors after conditioning runs. Specimens were re-tested after fatiguing.

Six methods of fixation were analyzed: Steinman pin with and without $\mathrm{Cl}$ incorporation; $\mathrm{CD}$ Horseshoe with and without $\mathrm{Cl}$ incorporation, Mayfield Loop with $\mathrm{C} 1 / 2$ transarticular screw fixation; and a custom designed occipito-cervical transarticular screw/plate system. Sublaminar techniques were extended to include $\mathrm{C} 3$ in the fusion construct whereas transarticular techniques incorporated the occiput, $\mathrm{Cl}$, and $\mathrm{C} 2$ only. 
Results: All methods of fixation provided significant immobilization in all specimens compared to the unconstrained destabilized state. Despite incorporation of an extra vertebral segment, sublaminar techniques performed more poorly as a function of applied load compared to screw fixation. Following fatiguing, these differences were more pronounced. The sublaminar techniques failed most prominently in flexion and extension and in axial rotation. Increased angular displacement was associated with loosening of the sublaminar cables through bone wear observed grossly.

Conclusions: Occipito-cervical fixation can be accomplished making use of screw techniques. Such methods decrease the number of vertebral segments necessarily incorporated into the fusion construct. Biomechanical testing demonstrates superior immobilization with screw fixation compared to sublaminar methods especially after fatigue.

\section{E-04}

An Evaluation of Multilevel Cervical Laminectomy in the Treatment of Cervical Spondylotic Myelopathy: A Study of Clinical Outcome in 44 Patients

SD Christie, J Spears, V Mehta, RO Holness (Halifax, Nova Scotia)

Background: Cervical spondylosis is a common disorder and $5-10 \%$ of patients clinically progress to symptomatic cervical spondylotic myelopathy (CSM) ultimately yielding to irreversible neurologic deficits. Surgical decompression, via anterior or posterior approaches, can often halt the progression of the myelopathy and sometimes improve neurological function. Although the anterior approaches have been reported superior to laminectomy in various studies, we propose that laminectomy is a safe and effective procedure for many patients.

Methods: A retrospective review of 44 patients undergoing multilevel cervical laminectomy for CSM at a tertiary care center was performed. All cases between the years 1990 to 1997 were reviewed. Radiologic evaluation included a combination of plain $\mathrm{x}$-rays, computerized tomography and/or magnetic resonance imaging. Clinical assessments were obtained via hospital charts, consultant's notes, letters from primary care physicians and personal contact via telephone. Outcomes, ambulatory and subjective, were classified as improved, deteriorated or unchanged. Ambulatory outcome was assessed using the Nurick Grading Scale (0-5). Subjective outcome was based on physician's assessment and patient satisfaction.

Results: Of the 44 patients reviewed 29 (65.9\%) were male. The mean age was 66.4 years (range $38-85$ years). The mean length of follow-up was 2.5 years (range 3 weeks to 8 years). In terms of ambulation $21(47.7 \%)$ patients improved their Nurick grade. Another $20(45.5 \%)$ were unchanged and $3(6.8 \%)$ were found to have deteriorated. Subjectively, 31 $(70.5 \%)$ improved post-operatively, whereas, $10(22.7 \%)$ were felt to have remained the same and $3(6.8 \%)$ were felt to be worse.

Conclusions: Cervical laminectomy for CSM is an efficacious procedure that, when indicated, can halt progression or improve myelopathic symptoms in more than $90 \%$ of patients.

\section{E-05}

Surgical Treatment Outcome in Patients with Cervical Spondylotic Myelopathy and Radiculopathy

\section{FJ Espinosa, K Amjadi (Kingston, Ontario)}

The long-term surgical treatment outcome of 64 patients with documented spondylotic cervical myelopathy and/or radiculopathy who were admitted at Kingston General Hospital between April 1986 and December 1995 was reviewed retrospectively. Thirty eight patients presented with myelopathy, while 26 patients presented with radiculopathy. There were 22 women and 42 men with ages ranging between 27 and 82 years. Fiftyfour patients were available for long-term follow-up. The longterm follow-ups ranged from 18 months to 11 years evaluated according to the Nurick Scale, and through telephone interviews using a standardized questionnaire.

There was no surgical mortality in this series and morbidity rate was $4.7 \%$. Early post-operatively $76.3 \%$ of the myelopathy group, and $84.6 \%$ of the radiculopathy group improved from surgery. However, upon long-term follow-up, $41.6 \%$ of the myelopathy group, and $62 \%$ of the radiculopathy remained improved. In myelopathy patients, developmentally narrow spinal canal, duration of symptoms before surgery of greater than one year, and the age of the patient greater than 55 years at the time of surgery, at the time of surgery, were found to be related to poor surgical outcome. For radiculopathy patients history of trauma, and the need for repeat surgery were related to poor surgical outcome.

\section{F. MOVEMENT DISORDERS}

\section{F-01}

\section{Biochemical Basis of Chronic Levodopa Complications in Parkinson's Disease}

A.H. Rajput, M.E. Fenton, H. Sitte, C. Pifl, O. Hornykiewicz (Saskatoon, Saskatchewan)

Background: Adverse effects (AE) wearing off (WO), dyskinesia (Dys), on-off $(\mathrm{OO})$, and freezing $(\mathrm{Fz})$ are reported in more than $80 \%$ of Parkinson's disease (PD) cases treated with levodopa (LD) for more than 6 years. Increasing LD dose controls WO, but aggravates Dys indicating that their pathophysiology is dissimilar. Striatal dopamine (DA) loss is the main abnormality in PD and LD corrects that. Difference in DA metabolism may explain AE patterns.

Objective: To study DA metabolism in PD cases with different $\mathrm{AE}$.

Methods: 7 frozen brains from PD patients and 5 control brains were analysed for DA and homovanillic acid (HVA).

Results: Significant reduction of DA levels and elevated DA/HVA ratio in the striatum, accumbens, external pallidum, internal pallidum and the subthalamic nucleus were detected. Higher ratio indicated more pronounced DA loss. In 4 patients 2 only WO and 2 only Dys - striatal DA levels were comparable, but HVA/DA ratios were much higher in the 2 WO $(23.3,20.8)$ compared to the 2 Dys $(15.5,12.2)$. 
Conclusion: Higher HVA/DA ratio indicates that DA is metabolised more rapidly in WO than in Dys. Our data suggest that one possible explanation for different $\mathrm{AE}$ is the rate of $\mathrm{DA}$ metabolism.

F-02

Correlation Between The Number of Substantia Nigra Cells and Dyskinesia, Wearing Off, and On Off in Parkinson's Disease

M.E. Fenton, R. Macaulay, A.H. Rajput (Saskatoon, Saskatchewan)

Background: Levodopa (LD) remains the best treatment for idiopathic Parkinson's disease (PD), but most patients develop adverse effects (AE) - dyskinesia (DK), wearing off (WO), and/or on off $(\mathrm{OO})$ - during treatment. WO is believed to result from the progressive loss of substantia nigra (SN) dopaminergic neurons leading to decreased capacity to store dopamine between doses and buffer changes in plasma LD concentrations due to intermittent dosing. The pathophysiology of DK is yet to be determined.

Method: SN counts were done blinded to LD complications in autopsy verified PD cases followed by one neurologist.

Results: Counts were done in 21 cases - 8 DK-only, 1 WOonly, $3 \mathrm{DK}+\mathrm{WO}, 1 \mathrm{WO}+\mathrm{OO}, 4 \mathrm{DK}+\mathrm{WO}+\mathrm{OO}$, and 4 with no LD complications. The mean number of SN cells in DK-only was $212, \mathrm{DK}+\mathrm{WO} 219, \mathrm{DK}+\mathrm{WO}+\mathrm{OO} 189$, and in cases with no complications was 229 . Statistical comparison revealed no significant difference among these groups.

Conclusions: Although there was a trend toward lower number of $\mathrm{SN}$ neurons in those with all types of $\mathrm{AE}$ compared to those with no $A E$, there was no significant difference among the subgroups. The pathophysiology of these phenomena cannot be explained based on neuronal loss alone.

\section{F-05}

Localization of Glutamate Receptors on Interneurons in Human Striatum: Possible Clues to Huntington's Disease Pathology

F Cicchetti, A Parent (Quebec, Quebec), TG Beach (Sun City, Arizona)

We recently reported the existence of a new class of interneurons characterized by their immunoreactivity for the calciumbinding protein calretinin (CR) in the human striatum. This class is composed of numerous medium-sized bipolar neurons and a smaller number of large multipolar neurons. Observations in the striatum of Huntington's disease (HD) patients revealed the selective sparing of the medium, but not of all large, CR+ striatal neurons in HD. Since glutamate receptor-mediated excitotoxicity has been implicated in the specific loss of striatal projection neurons, a central feature of the movement disorder HD, we have undertaken the present immunohistochemical study to examine the expression of different AMPA/KA glutamate receptor subunits on the $C R$ immunoreactive interneurons.
Preliminary results show that a significant proportion of the large-sized CR+ neurons display the GluR1 and GluR2/3 subunits compared to the GluR4 subunit that was only expressed on a small number of the latter neuronal population. A small porportion of medium-sized neurons showed immunoreactivity for GluR I whereas the majority expressed GluR2/3 and GluR4 subunits. The GluR5/6/7 subunit showed the most colocalization in both population of $\mathrm{CR}+$ interneurons. Since the GluR2 subunit has the ability to "shut down" $\mathrm{Ca} 2+$ influx through AMPA receptor channels, thus protecting cells from excitotoxicity, its specific expression among CR+ could be of interest. The study of metabotropic glutamate receptors also needs further attention since some antagonists of these receptors have been demonstrated to be powerful therapeutic agents. Their localization could unravel potential target sites in the control of motor symptoms. We believe that these anatomical data will help better understand the processes involved in the selective sparing of the medium-sized $\mathrm{CR}+$ striatal interneurons in HD.

\section{F-06}

\section{Estimating Survival in Dementia Using Prevalent Cases}

Asgharian M, Rouah F, Wolfson C, Wolfson D (Montreal, Quebec)

Background: Most studies of survival in dementia are based on follow-up of subjects ascertained in cross-sectional surveys. It is well known that in cross-sectional surveys, one is more likely to select into the study sample those individuals with long disease duration. This phenomenon is known as length-biased sampling and estimation of survival without taking this into account will likely result in biased estimates.

Methods: Using 5-year follow-up data on demented subjects ascertained in the first phase of the Canadian Study of Health and Aging (CSHA-1, 1991-1992), we examined this issue. The unadjusted estimated survival function based on the sample of 944 prevalent was compared to that obtained using a method that adjusts for length-biased sampling. The adjustment is easily carried out using standard statistical software.

Results: The unadjusted method resulted in an important overestimation of survival. For example, the unadjusted estimate of 7 year survival post-onset was approximately $60 \%$ as compared to $30 \%$ using the adjusted method.

Conclusions: Our results illustrate the importance of taking into account length-biased sampling when estimating survival. This presentation will discuss the results and suggest methods for assessing the impact of covariates on survival using prevalent cases.

\section{F-07}

Standard Neuropsychological Tests Do Not Predict Development of Alzheimer's Disease in Individuals with "Age-associated Cognitive Decline"

H. Chertkow, H. Bergman, C. Wolfson, L. Babins, N. Kelner (Montreal, Quebec) 
Background: When elderly individuals complain of mild memory loss, and demonstrate objective impairment in memory on age-normed tests, yet do not meet criteria for dementia, the term "age-associated cognitive decline" (hence AACD) has been applied. Neuropsychological tests alone or in combination have been proposed as possible measures to predict which of these individuals will progress to dementia.

Methods: 92 elderly individuals (mean age 74) meeting criteria for AACD, were enrolled in a longitudinal study of predictors. Among many measures taken, a full battery of age-and education standardized neuropsychological tests was administered.

Results: After mean 33 months follow-up, 42 of the cohort now met criteria for dementia (probable Alzheimer's Disease). Those progressing were somewhat older and had somewhat longer follow-up (mean 36 vs. 31 months). Those subjects progressing were somewhat more impaired at initial assessment in block design, delayed verbal and non-verbal memory, and picture naming. None of these scores alone or in combination, however, was robust enough to accurately predict progression.

Conclusions: Individuals meeting criteria for AACD have a high risk (46\%) of developing dementia over three year followup. Standard neuropsychological testing at presentation is unable to predict which individuals will deteriorate and who will remain stable.

\section{F-08}

Can Clinical Data Predict Progression to Alzheimer's Disease in Patients with Mild Memory Impairment?

\section{Fellows, H Chertkow, H Bergman (Montreal, Quebec)}

Background: The early diagnosis of Alzheimer's disease (AD) is becoming ever more important, particularly with the advent of new treatments for this condition.

Methods: Patients were drawn from a population presenting to a tertiary care centre memory clinic. Seventy patients meetings criteria for age-associated cognitive decline (AACD) (i.e. subjective and objective memory impairment but not meeting the criteria for dementia) were followed prospectively for a minimum of 2 years. Information obtained by history and physical examination on the initial visit was compared in those who eventually progressed to AD with those who did not progress.

Results: Twenty-six patients progressed to AD. These patients were slightly older at presentation than those who did not progress $(77 \pm 5$ vs $71 \pm 7$ yrs; mean $\pm S D)$. Both groups were similar in terms of baseline level of function, education and Mini-Mental Status Examination scores.

There were no significant differences between the 2 groups in the frequency of risk factors for atherosclerosis, smoking or alcohol use, family history of dementia, symptoms of depression, or subjective memory complaints. Recent functional decline or family concerns that the patient's memory was failing were not more common amongst those who developed AD. There was also no difference in the frequency of abnormalities on neurologic examination.

Conclusion: Information obtained by history and physical examination alone is not sufficient to predict progression to $A D$ in patients who meet the criteria for AACD on initial presentation.

\section{F-09}

\section{Does Atrophy of the Temporal Lobes Predict Decline to Dementia?}

\section{S. Murtha, T. Fitch, R. DelCarpio, H. Bergman, H. Chertkow} (Montreal, Quebec)

Background: Volumetric changes in the temporal lobe have been consistently found in patients with dementia of the Alzheimer's Type (DAT) when compared to age matched control subjects. We asked whether an increase in the temporal horn of the lateral ventricle volumes (THV) combined with a decrease in hippocampal volumes (HV) would also be found in a subgroup of patients diagnosed with aging associated cognitive decline (AACD). Patients in this group do not meet the criteria for probable DAT but have been found to be at a higher risk for subsequent development of DAT when followed longitudinally. Being able to predict those AACD patients at greater risk would have implications for prophylactic treatment, when such therapeutic modalities become available.

Methods: Magnetic resonance imaging (MRI) scans were performed on $23 \mathrm{AACD}$ subjects, $25 \mathrm{DAT}$, and 25 control subjects. In order to normalize the brain scans for size, orientation and position, they were transformed into stereotaxic (Talairach) space. An investigator blind to subject group measured the left and right temporal horns of the lateral ventricles, as well as the left and right hippocampal volumes. AACD subjects were followed annually for clinical reevaluation. At longitudinal followup (>1 year) 9 had progressed to meet clinical criteria for DAT (AACD-P); 14 had not progressed (AACD-NP).

Results: The ANOVAs between the 4 groups indicated that on average the DAT and the AACD-P group had enlarged bilateral THV and reduced $\mathrm{HV}$ as compared to the control subjects whereas the AACD-P group did not have THV or HV significantly different from the control group.

Conclusions: Analysis of volumetric changes in the temporal lobe showed the predicted changes in the AACD subjects who progressed. Therefore volumetric analysis of this area could act as a potential marker for early diagnosis of DAT.

\section{F-10}

\section{Parkinson's Disease and Comorbid Disorders}

\section{A Nataraj, AH Rajput (Saskatoon, Saskatchewan)}

Background: There has been controversy over whether there is decreased frequency of cerebrovascular disease (CVD) in Parkinson's Disease (PD). Reported decreased frequency of hypertension and smoking in PD and a theory that decreased dopamine may ameliorate ischemic cell damage to neurons provides rationale for this study.

Methods: All PD and essential tremor (ET) patients seen by 
one neurologist in Saskatoon are entered into a database. From this, 490 random PD patients and 290 age and sex matched ET patients were chosen. 490 age and sex matched controls were obtained from the records of two Saskatchewan family physicians. Records were assessed for various comorbid conditions. Symptomatic stroke and transient ischemic attacks (TIA) were included in CVD.

Results: PD had less current smokers than ET and controls. There were similar frequency of CVD and TIA. Clinically symptomatic stroke was less frequent in PD compared to controls (Pearson chisquare $=0.28$ ).

Conclusion: While CVD was not decreased in PD, clinically symptomatic stroke was less in PD. While it appears that decreased smoking plays a role, the idea that decreased dopamine and other neurotransmitters that may cause excitotoxic cell death cannot be discounted.

\section{F-11}

\section{Different Diagnostic Prevalence of Lewy Body Dementia According to 2 Sets of Criteria}

G Inglis, H Chertkow, M. Panisset, H. Bergman (Montreal, Quebec)

Background: Lewy Body dementia is now the second most prevalent dementing illness on neuro pathological examination. Clinically, this entity is now more recognized. To help clinicians in their diagnosis, several sets of criteria for LBD have been proposed.

Objective: To assess the prevalence of LBD using the 2 most common sets of criteria (the McKeith Criteria and the Consensus Criteria) in patients with a possible diagnosis of LBD.

Setting: 2 memory clinics of the Montreal region: the Jewish General Hospital and the Douglas Hospital.

Participants: Patients attending these memory clinic with possible diagnosis of LBD or Parkinson's disease with secondary dementia.

Measurements: Using the McKeith and Consensus Criteria, we reviewed the charts of patients with a diagnosis of possible LBD or Parkinson's disease with secondary dementia. We applied the 2 sets of criteria and assessed the difference in prevalence.

Results: 18 charts were reviewed. Using the McKeith Criteria, 6 patients met the criteria for LBD. 8 patients had a diagnosis of probable LBD with the Consensus Criteria and among these were the 6 patients who also met the McKeith Criteria. 9 patients had possible LBD according to the Consensus Criteria without fulfilling the McKeith Criteria. 1 patient did not meet any sets of criteria. Fluctuation of cognition is an obligatory finding according to the McKeith Criteria. In the Consensus set, progressive cognitive decline is mandatory, with fluctuating cognition being 1 of 3 prerequisites. It has been determined in the literature that fluctuating cognition is difficult to assess, which largely explains the difference in the prevalence of LBD between the 2 sets of criteria.

Conclusion: Using the Consensus Criteria we observe an increased prevalence of probable and possible LBD. Since fluctuating cognition is difficult to assess, we recommend using the Consensus Criteria.

\section{G. GENERAL NEUROLOGY}

\section{G-01}

\section{Evidence for Altered Glutamate Transport in Brain in Acute Liver Failure}

A Michalak, K Knecht, RF Butterworth (Montreal, Quebec)

An accumulating body of evidence suggests that modifications of glutamatergic function are implicated in the central nervous system complications of acute liver failure (ALF) (Michalak et al., Hepatol., 1995). It has been proposed that reduced uptake of neuronally-released glutamate contributes to the pathogenesis of the brain edema and hepatic encephalography in ALF. In order to address this issue, densities of binding sites for the glutamate transporter ligand [3H] D-aspartate were measured using quantitative autoradiography in the brains of rats with ALF resulting from portacaval anastomosis followed $48 \mathrm{~h}$ later by hepatic artery ligation. Densities of $\left[{ }^{3} \mathrm{H}\right] \mathrm{D}$-aspartate binding sites were selectively reduced by $20-65 \%(p<0.01)$ in frontal cortex, anterior cingulate, olfactory bulb \& tubercle, thalamus, hypothalamus and cerebellum at coma stage of encephalopathy in rats with ALF compared to control groups of animals. In order to further evaluate glutamate transporters, gene expression of recently cloned and sequenced astrocytic glutamate transporter GLT-1 was studied in brain preparations from rats at coma stage of ischemic liver failure and appropriate sham-operated controls. GLT-1 gene expression was studied using a cDNA probe and reverse transcriptase-polymerase chain reaction (RTPCR). Expression of GLT-1 RT-PCR product, normalized to glyceraldehyde-3-phosphate dehydrogenase was decreased in frontal cortex by $25 \%(\mathrm{p}<0.05)$ and cerebellum by $55 \%(\mathrm{p}<$ 0.01 ) of rats at coma stage of acute liver failure. Furthermore, decreased GLT-1 gene expression in frontal cortex coincided with a significant increase in extracellular concentrations of glutamate, measured using in vivo cerebral microdialysis.

Our study suggests: the decreased brain glutamate transport in acute liver failure is due to decreased expression of the glutamate transporter GLT-1; astrocytic reuptake of neuronallyreleased glutamate is reduced, resulting from GLT-1 transporter deficit; comprised neuron-astrocytic trafficking could contribute to the glutamatergic synaptic dysfunction and be responsible for the central nervous system complications characteristic of hepatic encephalopathy in acute liver failure. (Funded by MRC Canada).

\section{G-02}

Genetic Aspects of Charcot-Marie-Tooth Neuropathy in the French-Canadian Population: A Preliminary Study

N. Dupré, J. Puymirat, J.-P. Bouchard, D. Brunet (Quebec City, 
Quebec), M. Vanasse (Montreal, Quebec), B. Lemieux (Sherbrooke, Quebec)

Background: The objectives of this study are to evaluate the epidemiology of Charcot-Marie-Tooth (CMT) neuropathy in the French-Canadian population, using both clinical and molecular diagnosis.

Methods: Patients under study are recruited in neuromuscular clinics in the Province of Quebec. Diagnoses are based on clinical and electrophysiologic data, and confirmed by molecular analysis (search for duplication or deletion of $1.5 \mathrm{mb}$ segment on chromosome 17 by Southern Blot analysis, and identification of point mutations on the Po, PMP22, and connexin 32 genes by Single Strength Conformation Polymorphism).

Results: Sixty-five (65) families have been evaluated clinically. Thirty-seven (37) families were recruited out of the adult population, of which $30(81 \%)$ were of demyelinating types of CMT, and 7 (19\%) were of axonal types. Twenty-eight (28) families were from the pediatric population, of which $24(86 \%)$ were of demyelinating types, and 4 (13\%) of axonal types. Of the families for which molecular analysis was available, we identified two of X-linked dominant CMT (CMTX) type, with confirmed point mutations on the connexin 32 gene at Arg 142Trp, and Ser26Trp (previously unpublished).

Conclusion: We found similar proportions of axonal and demyelinating types of CMT as described in other populations, and report equivalent repartition of these types in both the adult and pediatric populations. Also, we report a previously unpublished mutation in the CMTX type.

\section{G-03}

Incidence of Concussion in High School Football Players of Northern Ohio and Pennsylvania

\author{
W. Langburt, N. Akhtar, K. O'Neill, B. Cohen (Toronto, \\ Ontario)
}

Introduction: In North America, football is the sport that accounts for most concussions. Gerberich et al. surveyed 3,063 high school football players in Minnesota in 1977. They found that $19 / 100$ players reported at least one concussion characterized by loss of consciousness or awareness.' These results have not been confirmed in subsequent studies. We hypothesized that the incidence is significantly higher than $19 \%$.

Methods: A survey was devised by the authors. Cantu guidelines $^{2}$ for concussion were used to define grades of head injury. The surveys were distributed by the athletic boards to 450 high school football players of districts in Ohio and Pennsylvania after the 1996/97 season. The surveys were anonymous. Athletes were requested to provide information about the number of head injuries sustained in the prior season, medical care sought, residual symptoms, and length of time required to stop play.

Results: 450 surveys were distributed, and 234 high school football players responded $(52 \%)$. The incidence of concussion in football players was $47 \%$ vs. a pre-determined rate of $19 \%$, representing a statistically significant difference $(p<.001)$. Multiple concussions were experienced by $35 \%$. Grade I concussion accounted for $87.8 \%$, grade II $9.9 \%$, grade III $2.4 \%$. Only 12 athletes were required to stop play for one or more games.

Conclusions: The incidence of high school football players sustaining a concussion is much higher than previously described. The majority of these are mild concussions. The effects of multiple, repeated low grade concussions concussions are not known and further research in this area is needed.

'SG Gerberich et al.Am Jnl Public Health.73:1370-75, 1983

${ }^{2}$ Cantu RC.Guidelines for return to contact sports after a cerebral concussion. Phys Sports Med 1986;14:75-83.

\section{G-04}

\section{Isolated Third Nerve Palsy Related to Cocaine Abuse}

D.K. Nguyen, A. Robillard (Montreal, Quebec)

Background: Use of cocaine has been associated with seizures, ischemic strokes, subarachnoid hemorrhages, and intraparenchymal hemorrhages. The most common clinical presentations are seizures, headaches, transient loss of consciousness, and focal neurologic symptoms or signs. Isolated third nerve palsy has, to our knowledge, never been associated with cocaine use.

Methods: A detailed report of the clinical presentation is given and results of paraclinical investigations are reviewed. We then discuss the etiology and possible pathophysiology of this clinical picture.

Results: Three days after having abused of cocaine, a 22year-old man experienced diplopia, photophobia, and right-sided headaches. Neurological examination was normal except for a right third nerve palsy. Extensive laboratory investigations were negative. Cerebrospinal fluid analysis did not reveal any abnormalitiies. Cerebral CT-scan and MRI were normal. Four-vessel catheter cerebral angiography and angioMRI were normal and did not demonstrate any aneurysm, vasospasm nor vasculitis.

Conclusion: Our patient represents to our knowledge the first case of isolated third nerve palsy due to cocaine use. An infarction at the midbrain level remains the most probable hypothesis. Mechanisms such as vasospasm, induction of thrombosis or vasculitis have been suggested previously. Cocaine use should be considered in the differential diagnosis of isolated third nerve palsy.

\section{G-05}

Increasing Incidence of Amyotrophic Lateral Sclerosis in British Columbia

Dean Johnston, Andrew Eisen (Vancouver, British Columbia)

Background: Neurologists with an interest in ALS have suspected that the incidence of this disorder in increasing. Three previous studies from USA, France and Sweden have confirmed this suspicion. A single ALS clinic serves the approximately 3.1 million population of British Columbia making it uniquely suited to a population-based study of ALS. 
Methods: A data base of all patients seen at the ALS clinic has been maintained since 1980 . We selected patients seen during the decade between 1986 to 1996 who met the El Escorial criteria for definite ALS to determine if there was an increasing incidence during this period.

Results: The number of cases seen during the decade was 530. It is estimated this exceeds $80 \%$ of all patients in the province who develop ALS. The point prevalence at December 3 Ist 1996 was 7.87 per 100,000 (245 cases) and the average age-adjusted annual incidence was 1.70 per 100,000 which steadily increased from 0.91 in 1986 to 2.15 in 1996 ( $p<0.001$ ).

Comment: The incidence of ALS has significantly increased in B.C. over the period 1986-1996. Possible reasons for this include longer survival of an aging population, better ascertainment, and the effect that drug trials have on attracting patients to the clinic. These factors did not change significantly between 1980 and 1985 and cannot account entirely for the subsequent increasing incidence. It is likely that environmental factor(s) in a genetically predisposed population is/are important in the etiopathogenesis of ALS.

\section{G-06}

\section{A Search for CSF Immunoglobulin Binding to Human Myelin Basic Protein in Non-multiple Sclerosis Patients}

K.G. Makus, D.T. Warren, I. Catz, K.G. Warren (Edmonton, Alberta)

Background: Autoantibodies to human myelin basic protein (h-MBP) are readily detected by a solid phase radioimmunoassay in both free and bound forms in CSF of patients with acute relapsing or chronic progressive phases of multiple sclerosis (MS). The major purpose of this report was to determine whether CSF immunoglobulin binding to human MBP could be detected in CSF of non-MS patients by the same method.

Method: From 1978 until $1998 \mathrm{CSF}$ from a total of 1720 non-MS patients was analyzed for the presence of immunoglobulin binding to human MBP.

Results: In this presentation the entire non-MS population (1720 patients) will be illustrated. In 1682 of these patients results were negative. In the remaining 38 patients or $2.2 \%$ of the total non-MS population studied, results were positive. The non-MS patients with positive results consisted of 14 patients with possible predisseminated MS. In the organic disease group there were 5 patients with inflammatory disease, 5 siblings with familial spastic paraparesis, 3 patients with hypertension and lacunar state, 2 with spastic paraparesis, 2 spinal cord tumors, 1 with cervical spondylosis, 1 with trauma, 1 with motor neuron disease, I with leukodystrophy, I with spinal stenosis, I with berry aneurysm and 1 with Wernicke-Korsakoff syndrome. The CSF immunoglobulin profiles of these 38 patients will be presented in detail.

Conclusion: CSF IgG binding to hMBP occurs in $1-2 \%$ of a non MS population compared to $90-95 \%$ of MS patients with active disease.

\section{G-08}

Human IFN- $\beta$ : The Biochemical Basis for the Difference in Biological Activity and Immunogenicity

SE Goelz, L Runkel, W Meier, A Whitty, M Karpusas, K Kimball, M Brickelmaier, C Muldowney, W Jones, B Pepinsky (Cambridge, Massachusetts)

Of the three recombinant IFN- $\beta$ s shown to be effective for treatment of multiple sclerosis, AVONEX ${ }^{(s)}$ (Biogen) and Rebif ${ }^{(w)}$ (Ares-Serono) are both IFN- $\beta-1$ a and are glycosylated with the natural sequence. IFN- $\beta-1 b$ (Betaseron ${ }^{\star}$, Berlex) is non-glycosylated, lacks the $\mathrm{N}$-terminal methionine and has a cys ${ }_{17} \rightarrow$ ser mutation. The reported specific activities, based on different assays and/or standards, differ: AVONEX ${ }^{(*)}, 200 \mathrm{MIU} / \mathrm{mg}$; Rebif $^{(4)}, 300 \mathrm{MlU} / \mathrm{mg}$; Betaseron ${ }^{(2)}, 32 \mathrm{MIU} / \mathrm{mg}$. As reported in MS trials, the immunogenicity of the IFN- $\beta$ s also differ. The incidences of neutralizing antibody formation are: Betaseron ${ }^{(*)}$ (SC,qod), 30-40\%; AVONEX ${ }^{(*)}$ (IM Ix/wk), 6\%; Rebif(w) (SC $3 \mathrm{x} / \mathrm{wk}), 23.8 \%(22 \mathrm{mcg})$ and $12.5 \%(44 \mathrm{mcg})$.

To accurately determine the relative potencies and immunogenicity of these products, comparative analyses were performed. In vitro side-by-side assays have specific activities of: $200 \mathrm{MIU} / \mathrm{mg}$ for AVONEX ${ }^{(1)}, 20 \mathrm{MIU} / \mathrm{mg}$ for Betaseron ${ }^{(1)}$ and $140 \mathrm{MIU} / \mathrm{mg}$ for Rebif ${ }^{\otimes}$. Of the known structural differences between AVONEX ${ }^{\oplus}$ and Betaseron ${ }^{\oplus}$, only the absence of glycosylation from IFN- $\beta$ was shown to affect in vitro activity. Results will be presented that show an increase in aggregation and sensitivity to thermal denaturation with deglycosylated IFN$\beta$. This tendency to aggregate may contribute to the 5-7 fold higher immunogenicity of Betaseron.

Conclusion: AVONEX ${ }^{\otimes}$ has the highest specific activity and lowest immunogenicity of the three commercially available recombinant IFN- $\beta$ s approved for the treatment of MS.

\section{G-09}

\section{Tnf Neutralization Induces An Increase In Relapses In} Patients With Multiple Sclerosis

The Lenercept Multiple Sclerosis Study Group \& The UBC MS/MRI Analysis Group, presented by D.W. Paty (Vancouver, British Columbia)

Broad, if circumstantial evidence from clinical laboratory and animal studies suggest that the pro-inflammatory cytokine TNF has a role in MS pathology. One hundred and sixty seven patients with RR and SP MS received Lenercept (sTNFR-IgG p55) at doses of 10,50 , and $100 \mathrm{mg}$ or placebo each month as part of a 48-week phase II study to test the safety and efficacy of a TNF neutralizing therapeutic strategy. Magnetic resonance imaging was performed at 8 time points, i.e., at screening, baseline, and every 4 weeks thereafter through week 24 . Analysis of the MRI results (uniquely newly active lesions, percent of active scans and burden of disease) revealed no statistically significant differences between the treatment groups. However, there was a trend, in most measures, for increased activity at the higher doses. In addition, the number of patients with exacerbations 
and the total number of exacerbations were greater in the active treatment groups. The time to first relapse was shorter in actively treated patients. The change in the EDSS score after 24 and 48 weeks of treatment did not differ among treatment groups. Transient episodes of flushing and dyspnea occurred after the injection of Lenercept at doses of 50 and $100 \mathrm{mg}$. An increase in the occurrence of headaches was noted at all doses.

Relapses by Treatment Group through Study Week 48

Lenercept

\begin{tabular}{lllll}
\hline $\begin{array}{l}\text { Group } \\
(\mathrm{n})\end{array}$ & $\begin{array}{l}\text { Placebo } \\
(43)\end{array}$ & $\begin{array}{l}10 \mathrm{mg} \\
(44)\end{array}$ & $\begin{array}{l}50 \mathrm{mg} \\
(40)\end{array}$ & $\begin{array}{l}100 \mathrm{mg} \\
(40)\end{array}$ \\
\hline $\begin{array}{l}\text { \# of pt with } \\
\text { relapses }\end{array}$ & 22 & 26 & 32 & 32 \\
\hline
\end{tabular}

Total \# of relapses ${ }^{2}$

38

57

49

${ }^{1}$ chi-sq $\mathrm{p}=0.007$

${ }^{2}$ Kruskal-Wallis Test $\mathrm{p}=0.009$

\section{G-10}

Treatment of Multiple Sclerosis-Related Fatigue with Aspirin

D.M. Wingerchuk, E.E. Benarroch, M. Rodriguez, (Rochester, Minnesota)

Background: Fatigue is the most common symptom of multiple sclerosis (MS). Available therapies have limited efficacy and do not provide insight into the pathophysiologic mechanisms of MS-related fatigue.

Methods: Eight MS patients with refractory fatigue were empirically treated with $1300 \mathrm{mg} / \mathrm{d}$ of aspirin because an apparent beneficial effect was noted in several prior patients utilizing moderate to high doses (975-1950 mg/d) of aspirin for other reasons.

Results: Seven of the eight patients reported significant improvement with a mean latency to maximal effect of 3 weeks. Follow-up ranged from 2 to 11 months with sustained effect in all improved patients. No significant adverse effects were reported.

Conclusions: Moderate doses of aspirin may ameliorate fatigue for prolonged periods in selected MS patients. Based upon these uncontrolled clinical observations, we propose a model of fatigue which integrates the effects of cytokines produced in MS lesions, the immunomodulatory effects of eicosanoids, and the neuroendrocrine axis. This hypothesis will be presented in further detail. A randomized, double-blind, placebo-controlled trial of aspirin for MS-related fatigue is in progress.

\section{G-11}

Treatment with Intravenous Immunoglobulin in Acute Disseminated Encephalomyelitis: Subsequent Recovery in Two Patients
D.J. Sahlas, S. Miller, M. Veilleux, G. Francis (Montreal, Quebec)

Background: Acute disseminated encephalomyelitis (ADEM) is an inflammatory, autoimmune, demyelinating disease of the CNS. Neurological deficits typically appear following viral illness or vaccination. ACTH, high-dose pulse steroids, and plasmapheresis have each been used as treatment with varying results. The use of intravenous immunoglobulin (IVIg) has not been previously investigated.

Methods: Patient 1 is a 24 year old female who developed spastic weakness and cognitive impairment 5-6 weeks following a febrile illness. Clinical deterioration continued despite treatment with high-dose pulse steroids. A 5 day course of IVIg $30 \mathrm{~g}$ daily administered 4 weeks following symptom onset was associated with clinical improvement. Patient 2 is a 38 year old female who presented with increased reflexes and cognitive impairment following a suspected viral illness. She also continued to worsen despite high-dose pulse steroids but showed improvement after IVIg. MRI in both patients showed multifocal white matter lesions.

Results: In both cases, treatment with IVIg was associated with a halt in the progression of the cognitive and neurological deficits and subsequent clinical improvement. Follow-up 6 months later revealed a continual improvement with no recurrence of symptoms.

Conclusions: IVIg should be further studied as a potential therapy in a controlled trial for patients with ADEM.

\section{H. CEREBROVASCULAR}

\section{H-01}

Cerebral Aneurysm Surgery Based on Preoperative CT Angiography

\section{G.B. Anderson, J.M. Findlay (Edmonton, Alberta)}

Objectives: To study the practice of cerebral aneurysm surgery for acute subarachnoid hemorrhage (SAH) based wholly on preoperative $\mathrm{CT}$ angiography.

Methods: In an ongoing prospective study, 90 consecutive patients with suspected intracranial aneurysms underwent CT angiography (CTA), digital subtraction angiography (DSA), or both, after an initial plain CT of the head. Based on plain CT and CTA the decision to proceed to DSA or directly to surgery was made.

Results: A total of 90 consecutive patients were studied. In 9 SAH patients both the CTA and following DSA were negative for aneurysm. Of 60 patients who underwent CTA before surgery, $28(50 \%)$ also underwent preoperative DSA. The decision to proceed to DSA following CTA was influenced by the location of the aneurysm (acomm a. and pcomm a. most commonly proceeded to DSA), but most importantly the quality of the CTA images. The sensitivity and specificity for aneurysm detection by CTA, as compared to DSA, in this group was $76 \%$ and $100 \%$, respectively. Eleven aneurysms (4 in one patient, 3 in two others) missed by CTA were $<4 \mathrm{~mm}$ in size. In $30(50 \%)$ 
patients aneurysm surgery was based on plain CT and CTA alone. The sensitivity and specificity for CTA in this group, as compared to postoperative DSA was $81 \%$ and $100 \%$, respectively. Eight aneurysms were not detected on the preoperative CTA ( 3 in one patient) all were $<4 \mathrm{~mm}$.

Conclusions: Aneurysm surgery based wholly on preoperative plain CT and CTA was possible in nearly $50 \%$ of patients in this series thus far, however, small aneurysms $(<4 \mathrm{~mm})$, especially of the juxtasellar ICA are at risk for being undetected by CTA. Nevertheless, the distinct advantage of CTA is of rapidly obtained images (an extra 10-15 min following plain CT), which when clearly demonstrating the ruptured aneurysm, can facilitate safe surgery without the time and dangers associated with DSA.

\section{H-02}

Endovascular Treatment of Intracranial Aneurysms Using Guglielmi Detachable Coils: The Value of Computed Tomographic Angiography

D. Tampieri, G. Mohr, M. Angle, R. Pokrupa, A. Sadikot, L. Jacques, R. Leblanc, A. Olivier, D. Melançon (Montreal, Quebec)

Background: The aim of this presentation is to report our experience with endovascular treatment of intracranial aneurysms at the Montreal Neurological Hospital (MNH) during the past three years. The role of CTA in tailoring the procedure of choice (endovascular versus surgical) will also be addressed.

Methods: Since August 1995, 38 endovascular procedures were performed on 36 patients at the MNH for endovascular treatment of intracranial aneurysms. Out of 38 procedures, 36 aneurysms were treatable. In one case, catheterization of the aneurysm was unsuccessful, and in the other, at the time of the angiogram the aneurysm was not found suitable for GDC. These procedures are performed under general anaesthesia and full heparinization.

Results: We obtained complete occlusion of the aneurysm in $69 \%$ of the cases immediately. In $22 \%$ of the cases, we had more than $90 \%$ of the aneurysm sac and in 3 cases the occlusion was only $70 \%$ of the sac. We had no recanalization. Complications were separated by procedure and disease-related causes. There were $3(7 \%)$ cases ( 2 transitory and one permanent $(2 \%)$ ) of procedure-related complications and no mortality. Final patient outcome was $15 \%$ morbidity and $13 \%$ mortality including all grades keeping in mind that $18 / 38$ patients in our series were $\mathrm{SAH}$ grade 3 and above.

Conclusions: Endovascular treatment with GDC plays a very important role in the current management of intracranial aneurysms. CTA may help in the treatment planning.

\section{H-03}

Transcranial Doppler in the Evaluation of Intracranial Aneurysms: Preliminary Study

S. Fontaine, D. Tampieri, D. Melançon, F. Cattin, R. Leblanc (Montreal, Quebec)
Learning Objective: To assess the value of transcranial doppler (TCD) in the evaluation of intracranial aneurysms in view of endovascular versus surgical treatment.

Methods: Six patients harbouring one intracranial aneurysm underwent colour code TCD, power TCD, and in 3 cases, TCD with injection of contrast (Levovist, Berlex, $13 \mathrm{ml}$ of $300 \mathrm{mg}$ microparticles $/ \mathrm{ml}$ ) was performed. All patients underwent spiral CTA and DSA prior to treatment and the modalities were compared.

Results: TCD demonstrated aneurysms in all patients in our series. In one patient, the follow-up study clearly demonstrated the residual aneurysm (basilary tip) around hyperechoic GDC coils which were well visualized, causing no artifacts. Doppler evaluation of surrounding vessels is feasible. In 2 patients Levovist made for better assessment of the aneurysm and adjacent arteries. An 8mm aneurysm of the ACA was seen only after contrast enhancement.

Conclusions: It is possible to study aneurysms of the circle of Willis with TCD especially if contrast is used. The correlation with DSA and CTA was good. The clinical impact has yet to be confirmed although the follow-up of endovascular treatment is promising.

\section{H-04}

The Value of Transcranial Doppler Ultrasonography in the Diagnosis of Cerebral Vasospasm following Aneurysmal Subarachnoid Hemorrhage

Y. Y. Vora, J. M. Findlay, M. Suarez-Almazor, D. E. Steinke (Edmonton, Alberta)

Background: The role of transcranial Doppler (TCD) in the diagnosis and management of vasospasm following subarachnoid hemorrhage is controversial. The predictive value and correlation of TCD with angiographic vasospasm (AV) is presented.

Methods: A retrospective review of all patients admitted between Jan 1994 and Jun 1997 with aneurysmal SAH and who had a postoperative angiogram during the vasospasm risk period (days 4-21), was conducted. Time-averaged middle cerebral artery velocities were compared with AV (on the follow-up angiogram) judged by a neuroradiologist as being none $/ \mathrm{mild} / \mathrm{moderate} / \mathrm{severe}$. The neuroradiologist was unaware of the TCD results. Prophylactic hyperdynamic therapy (HT) was frequently used.

Results: Among 102 patients, the Spearman correlation coefficient between the highest velocity pre-angiogram and $A V$ was $0.51(p<0.001)$. Using the velocity on angiogram day, it was $0.55(\mathrm{p}<0.001)$. The highest pre-angiogram velocity, in those with moderate-severe AV was on average 196 vs. $122 \mathrm{~cm} / \mathrm{s}$ in those with none-mild AV ( $p<.001)$.

Using $120 \mathrm{~cm} / \mathrm{s}$ as the TCD definition of vasospasm, the correlation with none-mild vs. moderate-severe $\mathrm{AV}$ was poor (Kappa 0.15 ), sensitivity $94 \%$, specificity $54 \%$, positive predictive (PPV) and negative predictive (NPV) values $16 \%$ and $99 \%$ respectively. Using $160 \mathrm{~cm} / \mathrm{s}$, Kappa value was 0.40 , sensitivity $75 \%$, specificity $87 \%$, PPV $34 \%$, NPV $97 \%$. The highest FV 
during $\mathrm{HT}$, was on average $150 \mathrm{vs.} 129 \mathrm{~cm} / \mathrm{s}$ during no HT $(\mathrm{p}<.001)$. HT use was not correlated with the presence of moderate-severe $\mathrm{AV}(\mathrm{p}=0.12)$.

Conclusions: Routine use of HT may elevate absolute FVs thereby serving as a confounder. Consequently despite moderate overall correlation between TCD and $\mathrm{AV}$, the positive predictive value of absolute FVs for the purposes of clinical decision making in the individual patient can be poor.

\section{H-05}

\section{Intra-Operative Doppler Ultrasonography during Cerebral Aneurysm Surgery}

AM Kaufmann (Calgary, Alberta), JR Balzer (Pittsburgh, Pennsylvania)

Avoidance of arterial compromise during cerebral aneurysm clip obliteration is essential, and has traditionally been assessed by visual inspection, neuro-physiological monitoring, and intraoperative angiography. A recent experience with intra-operative Doppler ultrasonography to determine arterial patency is presented.

We reviewed a consecutive series of 107 cerebral aneurysms surgically treated between June 1994 and December 1997 (AMK). A $20 \mathrm{MHz}$ Doppler probe was used in 48 cases to insonate 176 associated cerebral arteries, before and after each clip placement. Vascular patency was ultimately achieved in all vessels following permanent aneurysm clip obliteration. However, in 6 cases (12\%) clip adjustments were made in reply to flow insufficiencies that were insonated despite the visual appearance of adequate initial clip placement. Furthermore, in five cases $(10 \%)$ mechanically induced vasospasm was treated when Doppler signals were reduced from pre-clip measurements. No patients demonstrated neuro-physiological, clinical or radiographic evidence of infarction related to the vascular territories of the insonated vessels. Intra- or post-operative angiography was also performed in 8 cases, and confirmed vascular patency and complete aneurysm obliteration.

Intra-operative Doppler ultrasonography provides a confident means of determining arterial patency before and after clipping of cerebral aneurysms. This rapid, non-invasive and cost-effective technique may facilitate optimal clip placement, as concerns regarding potential compromise of associated vessels may be immediately and directly assessed.

\section{H-06}

\section{Prognosis Factors In Poor-Grade Patients With Aneurysmal Rupture}

Bojanowski M.W., Demers J., Tétreault M.-H. (Montréal, Québec)

Background and purpose: While the management after an aneurysmal rupture in good-grade patient is relatively standardized, the approach for poor-grade (Hunt \& Hess grade IV-V) patient is more controversial. This is mainly due to the fact that there is no accurate outcome predictor available for this group associated with a worse prognosis. We reviewed our experience to define which factors are reliable to predict outcome in these patients.

Material and Methods: We retrospectively analyzed a series of all 75 poor-grade patients admitted by the senior author (M.W.B.) from 1990 to 1997. Patients' charts, CT scan and angiographic studies were reviewed. Aggressive patient management was uniformed including early surgery, vasospasm therapy, rigorous control of ICP as well as physiological parameters, in the intensive care unit. The outcome was assessed according to the Glasgow outcome scale.

Results: In this series of 75 patients, 43 over 48 grade IV patients were operated while 15 over 27 grade V patients were admitted in the operating room. Surgery was withheld in patients with absence of brain stem function or with extensive brain injury by an intracerebral hematoma. All unoperated patients eventually died. The outcome was favourable (G.O.S. 1 and 2) in 21/43 grade IV patients (49\%) and in $4 / 15$ grade $V$ $(27 \%)$ operated patients. Solely, gender, age or neurological status could not predict the outcome, neither could the presence of hydrocephalus. However, intracerebral hematoma $(p=0.01)$, ischemic lesions on the CTscan $(\mathrm{p}=0.03)$ and intractable ICP $(\mathrm{p}=0.04)$ was statistically related to a poor prognosis.

Conclusions: Clinical status on admission including age and neurological condition can not predict the outcome in poorgrade patients. Treated rapidly, hydrocephalus does not worsen the prognosis. We recommend aggressive medical and surgical approach for these patients unless there is no brain function or extensive brain injury by an intracerebral hematoma. Ischemic lesions and intractable ICP are however significant determinants in the post-operative period.

\section{H-07}

Surgery of Symptomatic Arteriovenous Malformations: A Decision Analysis

\section{R Leblanc (Montreal, Quebec)}

Introduction: Despite a greater awareness of cerebral arteriovenous malformations (AVM) since the advent of high resolution computed tomography (CT) scanning and magnetic resonance imaging (MRI), the management of cerebral AVMs remains empirical. We have used decision analysis to place management of AVMs on a more analytical basis.

Methods: The decision analysis model of Levey et al ( $\mathrm{N}$ Engl J Med 1983) that we previously applied to familial cerebral aneurysms (Leblanc et al, Neurosurg 1994) and to unruptured cerebral aneurysms (Leblanc \& Worsley, Can J Neurol Sci, 1995) was used to determine the benefit in years of survival free of sequelae resulting from the surgery of symptomatic cerebral AVMs over natural history. Based on the review by Heros and Tu (Neurology 1987) we took 3\% as the annual risk of rupture (r) $56.8 \%$ as the risk of death or disability with rupture (M) and $15.2 \%$ as the risk of surgery (S). Benefit was calculated from the equation $\mathrm{L}\left\{\left[1-(1-r)^{\mathrm{L}}\right] \mathrm{M} / 2-\mathrm{S}\right\}$ for life expectancy (L) corresponding to each quinquennial age group from age 15 to 85 years (Life Insurance Fact Book, 1990). Sensitivity analysis was 
performed to take into account various values for " $r$ ", "M", and " $\mathrm{S}$ " specific for each grade fo the AVM according to the grading system of Spetzler and Martin (J Neurosurg 1986).

Results: A gain of at least one year of survival free of neurological sequelae is achieved by surgery compared to natural history for patients whose life expectancy is approximately 33.5 years corresponding to approximately age 53 for women and 48 for men. Greater benefit is achieved with increased life expectancy (younger age) and with lower surgical risk (lower AVM grade).

Conclusion: Surgery of symptomatic cerebral AVMs achieves an increased survival over natural history of at least one year free of neurological sequelae in patients whose life expectancy is $\mathbf{3 3 . 5}$ years or more, using our baseline assumptions. Using the above equation the corresponding life expectancy producing this benefit can be calculated to account for the specific risk associated with various AVM grades in specific patients.

\section{H-08}

Partial Therapy for Brain Arteriovenous Malformations: Impact on Natural History of Hemorrhage

PJ Porter, K terBrugge, RA Willinsky, WJ Montanera, M Schwartz, M Tymianski, MC Wallace (Toronto, Ontario)

Background: The natural history of brain arteriovenous malformations (BAVMs) is not known. It is presumed that any residual portion of the BAVM seen after therapy imparts a continued risk of hemorrhage. This study was undertaken to determine the incidence of hemorrhage in a prospective cohort of patients with partial BAVM therapy to compare with untreated lesions.

Methods: Consecutive patients with a BAVM were followed prospectively between 1989-1996. Data were collected on type and timing of hemorrhage. Angiography was used to determine cure. Kaplan-Meir estimates (95\% confidence intervals CI) were calculated for hemorrhage in patients with partial therapy and observation. Multivariate analysis was used to adjust for differences in location, grade and angioarchitectural features.

Results: There were 390 patients with a mean age of 36.4 years. Spetzler grade was I-III in $304(78 \%)$ and IV-V in 86 (22\%) patients. Follow-up was obtained in $97 \%$ of patients. Treatment modalities included: no therapy $82(21 \%)$, surgery 65 (17\%), embolization $76(19 \%)$, radiosurgery $69(18 \%)$ and combination of the above $98(25 \%)$. Hemorrhage occurred in 14 patients with untreated BAVM's over 366 patient years of observation, compared to 24 events after partial therapy during 670 patient years. Kaplan-Meir estimates of bleeding were $17.4 \%$ (95\% CI 7.3-27.5\%) and $16.7 \%$ (95\% CI 9.0-24.3\%). No significant difference in hemorrhage risk was found using the Cox model adjusted for confounding BAVM features (relative risk $0.91, \mathrm{p}=0.83$ ).

Conclusion: In this large prospective series, there was no indication that partial treatment reduced the risk of bleeding compared to untreated lesions, considering the adjustment for confounding variables. The average annual risk of hemorrhage from a BAVM remained at $3.4 \%$.

\section{H-09}

\section{Impact of Therapy for Brain Arteriovenous Malformations on Seizure Frequency}

AJ Farragos, K terBrugge, RA Willinsky, WJ Montanera, M Schwartz, M Tymianski, MC Wallace (Toronto, Ontario)

Background: Control of seizures post treatment is a significant concern for patients considering therapy of brain arteriovenous malformations (BAVMs). The purpose of this study was to review seizure control in a series of BAVMs to determine outcome after therapy or no treatment at all.

Methods: Patients with seizures and a BAVM followed prospectively in our clinic with a minimum of 2 years follow-up were selected $(n=145 / 390)$. Information was obtained through clinic visits or by telephone survey. Seizure control was recorded by Engel classification.

Results: Treatment for the BAVMs included surgery (30), embolization (40), radiotherapy (30) and conservative therapy (45). Follow-up $(133 / 145,92 \%)$ was $4.3,6.8,4.5$ and 5.8 years respectively. Seizures were sporadic in $127 / 145(88 \%)$. After therapy, $32 / 80(40 \%)$ patients with sporadic seizures were cured, and $42 / 80(53 \%)$ were unchanged. Seizures increased in $6 / 80$ (7\%). No therapy of the BAVM left $29 / 37$ (78\%) seizure frequency unchanged.With significant pre-therapy seizures, 10/12 (83\%) patients were seizure free or improved after therapy . All treatment modalities improved seizure control.

Conclusion: In patients with BAVMs, seizures are usually sporadic in frequency. The treatment by any modality results in cessation or no change in sporadic seizures in the majority of patients. When pre-therapy seizure frequency is significant, post therapy seizure control is excellent.

\section{H-10}

Flow-Related Aneurysms Associated with Arteriovenous Malformations - Effect of Clinical Course and Natural History

GJ Redekop, K TerBrugge, R Willinsky, W Montanera (Toronto, Ontario)

Background: Flow-related aneurysms associated with arteriovenous malformations(AVMs) arise proximally in relation to the Circle of Willis or distally on feeding arteries. We propose an anatomical classification of AVM-associated aneurysms and document the effect of AVM therapy on their natural history.

Methods: Clinical charts and radiologic studies of patients with AVMs and associated aneurysms in the University of Toronto AVM Database were reviewed.

Results: Flow-related aneurysms were identified in 71 (11.3\%) of 630 patients with AVMs. The presentation was hemorrhage from the aneurysm in $12(17 \%)$ and hemorrhage from the AVM in $15(21 \%)$. Seventeen patients underwent angiography after AVM cure (mean 2 years). Of 23 proximal aneurysms, $18(78.3 \%$ ) were unchanged, 4 (17.4\%) were smaller, and $1(4.3 \%)$ disappeared, while 4 of 5 distal aneurysms regressed completely and 1 was unchanged. Sixteen patients underwent angiography after partial AVM treatment (mean 
3.8 years). In cases with less than $50 \%$ reduction of the AVM, no aneurysms regressed, while 2 enlarged and bled. In cases with greater than 50\% reduction of the AVM, 2 of 3 distal aneurysms disappeared, while 5 proximal aneurysms were unchanged.

Conclusions: Flow-related aneurysms present with hemorrhage almost as frequently as their associated AVMs. If the AVM is treated first, distal aneurysms usually disappear with substantial or complete nidus elimination, while proximal aneurysms rarely change even with curative AVM therapy.

\section{H-11}

\section{Non Aneurysmal Subarachnoid Hemorrhage}

Adada B., Bojanowski, M.W. (Montréal, Québec)

Background and purpose: Fifteen percent (15\%) of patients with subarachnoid heamorrhage (SAH) have normal angiograms. They fare better than patients with demonstrated aneurysms. In patients with a normal angiogram and accumulation of blood in the cisterns around the midbrain (perimesencephalic non aneurysmal SAH) outcome is thought to be better than in patients with normal angiograms and blood in another distribution. We wanted to ascertain this concept through our own experience.

Material and Methods: Patients for this study were selected from a consecutive series of patients with SAH evaluated between January first 1990 and March thirty first 1997. All patients with a SAH confirmed by a CT scan or a spinal tap and a normal angiogram were included in our study. The Hunt and Hess clinical grade, the Fisher radiological grade upon admission, as well as the development of any complications were noted.

Results: Six hundred and sixty eight (668) patients were evaluated for SAH. 55 patients had normal angiograms and were the object of this study. Among those, thirty four (34) had a perimesencephalic distribution, twenty one (21) an aneurysmal distribution. Of the patients with perimesencephalic distribution, four (4) had hydrocephalus necessitating a shunt, two (2) presented a motor deficit, one (1) had seizures and two (2) died. Patients with a motor deficit and those who died were all grade III of Fisher upon presentation. In the group of patients presenting with an aneurysmal distribution of bleeding, one (1) had seizures, one (1) had hydrocephaleus, one (1) had a motor deficit and one (1) died during hospitalisation.

Conclusions: Patients with perimensencephalic bleeding seem to develop as much complication as patients with other paterns of hemorrhage. This seems particularly true for patients with grade III of Fisher. Thus we recommend agressive medical management and vasospasm prevention for this group of patients.

\section{NEUROPHYSIOLOGY}

\section{I-01}

Electromyography Related Anxiety and Pain: A Prospective Study

MMS Jan, TJ Benstead (Halifax, Nova Scotia)
Electromyography (EMG) is a useful but sometimes painful test. Many patients worry excessively about its painful nature.

We studied anxiety levels of EMG patients to assess contributing factors . Patients completed a structured questionnaire including the State-Trait Anxiety Inventory prior to testing. Seriously ill patients were excluded.

Seventy-nine patients (ages 19-72 years - mean $43 \pm 12.6$, female:male - 2:1) were studied. Needle EMG was performed on $39(49 \%)$. Thirty-five (44\%) had high anxiety levels. High anxiety was more likely if the patient was worried about the test $(\mathrm{p}<0.001)$, or other concerns excluding the diagnosis $(\mathrm{p}<0.001)$, or was taking an anti-psychotic or anxiolytic drug $(\mathrm{P}=0.008)$. Knowledge of the testing procedure, did not affect anxiety levels.

Those who believed the test would be painless were 2.7 times more likely to have normal anxiety levels $(95 \% \mathrm{CI} 1-8)$. Only 7 (9\%) patients felt that the test was worse than expected, most of whom had technical difficulties during nerve conduction studies $(\mathrm{P}=0.007)$.

Anxiety levels in EMG patients correlate with worry about the test or other concerns held by the patient and were not affected by prior understanding of the test. The experience of most patients in the EMG lab is better than anticipated.

\section{I-02}

\section{Slowly Progressive Adult Onset Motor Neuron Disease Presenting as a Bi-brachial Amyotrophy}

M. Strong, B. Watson, N. Tasdemir, J.D. Brown (London, Ontario)

ALS is a progressive neurodegenerative disorder characterized by weakness and wasting throughout the bulbar and cervical to lumbosacral regions, with signs of corticospinal involvement. Survivorship depends upon the regions affected and is age dependent with average survivorship of 5 years. Rare instances of long-term survivors have been reported, predominately in the younger males.

We describe 6 cases ( 5 male, 1 female) with a mean age of symptom onset of 45.5 years (range, 32 to 53 years) presenting initially with asymmetrical bi-brachial weakness and wasting, pyramidal tract signs and fasciculations. Cranial and lower limb motor examinations were normal. Needle electromyography revealed fibrillation potentials, positive sharp waves and large amplitude polyphasic motor units in a bi-brachial distribution. Fasciculations were seen in both the upper and/or lower extremities in 4 cases. To date, all cases have survived on average 5.5 years, with persistent marked bi-brachial amyotrophy and predominately lower limb spasticity.

Regionally accentuated forms of motor neuron disease have been recognized but are usually restricted throughout the first three decades of life. Our cases illustrate a later, more benign form of motor neuron disease with clinical and electrophysiological results not typically diagnostic for ALS or anterior cervical cord syndrome in the adult population. 


\section{I-03}

Continuous EEG Monitoring In A Patient With Massive Carbamazepine Overdose

D. A. DeRubeis, G. B. Young (London, Ontario)

We report a case of a 32 year old woman with generalized nocturnal tonic-clonic seizures who took an intentional overdose (OD) of carbamazepine (CBZ). At presentation to a peripheral hospital serum CPZ was $360 \mu \mathrm{mol} / \mathrm{L}$. After an asystolic cardiac arrest of unknown duration, she was transferred to our hospital.

Continuous EEG (CEEG) monitoring was instituted from the time of presentation.

Examination revealed a deeply unconscious patient with multifocal stimulus sensitive myoclonus. Pupils were fixed and dilated with absent corneal and oculovestibular reflexes.

Initial CEEG recordings showed a burst-suppression pattern with bursts containing abundant epileptiform activity. She was treated with phenytoin, diazepam, midazolam, and valproic acid. The EEG evolved to continuous polymorphic delta wave activity. Hemoperfusion was undertaken with a decline in serum CBZ levels to $150 \mu \mathrm{mol} / \mathrm{L}$. Unfortunately she developed ARDS and hypoxemia followed by a decrease in EEG voltage and died.

Postmortem examination revealed no evidence of neuronal ischemia and well preserved cortical and subcortical structures.

This case is instructive because it: 1) demonstrates that massive CBZ OD causes paradoxical seizures and cortical hyperexcitability and may mimic brain death, 2) illustrates the value of CEEG monitoring in following the course and treatment in an $O D$ and 3 ) is the first report of a massive CBZ OD with CEEG monitoring and postmortem examination.

\section{I-04}

\section{Seizures in the Elderly}

\section{A. Holt-Seitz, E. Wirrell (Saskatoon, Saskatchewan)}

Purpose: To determine the etiology and natural history of new-onset seizures in elderly patients.

Methods: EEG records for the north-central region of Saskatchewan, between 01/94 and 12/95 were reviewed to identify all adults aged 60 years or older, with new-onset seizures. Information on demographics, seizure type, etiology, EEG and neuroimaging studies, anti-epileptic treatment and course of epilepsy was obtained by review of medical records and interview with the patient and/or family member. Seizure etiology and cause of death were determined for deceased patients.

Results: Of 88 eligible subjects, 61 (69\%) were contacted for follow-up, $19(22 \%)$ were deceased, 4 (5\%) each refused participation or were lost to follow-up. Fifty three of $61(87 \%)$ patients presented with partial or secondarily generalized seizures. Epileptiform discharge was seen in 25/61 (41\%) initial EEG recordings. CT scans were abnormal in 34/61 (54\%) cases. Fifty four $(89 \%)$ patients were treated with anti-epileptic medication and seizure control was usually successful. Predictors for ongoing seizures were more than 3 seizures at presentation, epilepti- form activity on initial EEG and discontinuation of anti-epileptic medication for lack of efficacy.

Seizures were idiopathic in $36 / 80(45 \%)$, and due to stroke in $17 / 80(21 \%)$.

Conclusion: Prognosis of new-onset seizures in elderly patients is favorable as long as seizures are not symptomatic of a life-threatening disorder.

\section{I-05}

Antebrachial Cutaneous Nerve: An Anatomical Study and Its Clinical Implications

L. Jacques, R.L. Tiel, D. Kline, J. Park (Montreal, Quebec and New Orleans, Louisiana)

Introduction: Understanding the anatomy of the medial and the lateral antebrachial cutaneous nerve (ABCNs) is important for various reasons. Firstly, the ability to correctly interpret the clinical examination, secondly, its role as a source of graft material for nerve operations, and finally, their exposure for injuries in the distal arm and forearm.

Method: 10 cadaver specimens provided 20 upper extremities for dissection. Supra and infraclavicular and distal arm surgical dissections were used to reveal the $\mathrm{ABCN}$ s from $\mathrm{C} 8-\mathrm{T} 1$ roots to their identifiable terminations in the distal forearm.

Results: The origin of the nerves from the brachial plexus occurs usually from the medial cord but individual variations were noted and origin from either the $\mathrm{T}-1$ root or lower trunk were also encountered. Recognizable branching patterns were associated both with the relationship of the ABCNs to the antecubital vein and at the epicondyle level. A bifurcation of the nerve was found in the distal arm. The caliber of the nerves varied between 3.5 to $4.0 \mathrm{~mm}$ prior the bifurcation and 1.0 to 2.0 $\mathrm{mm}$ after division. No discernable relationship of the $A B C N s$ to the biceps tendon was appreciated.

Discussion and Conclusions: Our results demonstrate the variability of $\mathrm{ABCNs}$ origin and document recognizable branching patterns of the nerve. Surgical planning will be assisted by understanding the branching patterns of the nerves. Improper venipuncture site selection may be avoided and the proper administration of local anestheetic blocks will also be facilitated. Our study offers no support to compression of the $A B C N s$ by the biceps tendon at the elbow vs recognizable entrapments.

\section{I-06}

Usefulness of Electrodiagnostic Studies in Early Diagnosis of Botulism

Y. Al-Said, M. Veilleux (Montreal, Quebec)

Typically, 12-36 hours following ingestion of botulinum toxin, there is cranial nerve involvement and subsequently, weakness of respiratory and limb muscles. Abdominal pain, vomiting, paralytic ileus, dry mouth and dilated pupils are also common features.

Between 1990 and 1996, 4 cases of botulism were admitted 
to the Montreal General Hospital and all presented with symptoms of acute limb weakness. Nerve conduction studies and repetitive nerve stimulation were performed in all of them and single fibre EMG in I patient. Serology for botulinum toxin in 3 patients was positive for type $\mathrm{E}$ botulinum toxin.

Nerve conduction studies (NCS) revealed markedly reduced motor action potential (MAP) amplitudes in the arms and legs but normal sensory nerve action potential amplitudes. On $2-\mathrm{Hz}$ repetitive stimulation of the ulnar nerve at rest, there was MAP amplitude decrement in 3 patients and no change in 1 . However, immediately following a 60 -second exercise, there was increment better seen during and after a $20-\mathrm{Hz}$ repetitive nerve stimulation. Single fibre EMG in 1 patient showed increased jitter and blocking.

Electrophysiological testing is an important tool for early diagnosis of botulism and can help to differentiate botulism from other causes of acute neuromuscular weakness.

\section{$\mathbf{I - 0 7}$}

Assessment of the Usefulness of the Fused Words Dichotic Listening and Tachistoscopic Tests for Language Lateralisation: Sensitivities, Specificities and Likelihood Ratios

J.T. Butler, M.C.S. Harnadek, S. Wiebe, W.T. Blume (London, Ontario)

Aims: To assess the usefulness of Dichotic Listening and Tachistoscopic tests for language lateralisation.

Methods: All patients undergoing bilateral Sodium Amytal, Fused Words Dichotic Listening and Tachistoscopic testing since 1991 as a prelude to epilepsy surgery were included. Amytal testing was performed to assess the risks to memory. Language representation by Amytal testing was categorised as left, right or bilateral. Dichotic Listening MISER Scores and Tachistoscopic Scores were each divided into four categories. Sensitivities, specificities and likelihood ratios were derived for each of the four categories.

Results: 30 of 49 patients undergoing both Amytal and Dichotic Listening testing had scores on the latter that permitted designation of either left hemisphere language representation (specificity $=99 \%$; likelihood ratio $=18$ ) or atypical language representation $($ specificity $=97 \%$; likelihood ratio $=21$ ). 47 patients had both Amytal and Tachistocopic testing. Scores on the latter did not assign language lateralisation reliably (likelihood ratio $<3$ in all categories).

Conclusion: The Fused Words Dichotic Listening test is a very useful test for language lateralisation while Tachistoscopic testing is not.

\section{I-08}

The Effect of Reversible Inactivation of Motor and Somatosensory Cortex on Independent Finger Movements, and Precision Grip Force Control in Monkeys

A. M. Smith, T. Brochier, M-J. Boudreau, M. Paré (Montréal, Québec)

Methods: Monkeys were trained to use a precision grip to lift and hold an object in a fixed position for 2.0 seconds. The computer controlled object measured both the grip and lifting forces and vertical position. The movements were recorded on video tape. An initial mapping of the receptive fields, and responses to microstimulation of the contralateral hand delimited the extent of the thumb and index finger representation in the somatosensory and motor cortex. Cortical inactivations were achieved with two $1.0 \mu \mathrm{l}$ injections of the GABA agonist, muscimol $(5 \mathrm{mg} / \mathrm{ml})$, into the thumb and index regions of either the pre- or postcentral gyrus.

Results: Although inactivations of both regions produced profound deficits in hand dexterity, they were readily distinguishable from each other. Motor cortex injections produced weakness in grip strength but no paralysis which appeared to be mainly a loss of independent finger movements reminiscent of the effects of bulbar pyramidotomy reported by Lawrence and Kuypers (Brain 91:1-14, 1968). The monkeys were unable to lift and maintain a $30 \mathrm{~g}$ weight for 2 seconds and the grip force peaks and rates of application were reduced. The effects seemed strongest on the intrinsic hand muscles, although a marked effect on finger extension was also seen. Even under visual control the monkeys could not use independent finger movements to extract pieces of food from small wells. Inactivation of somatosensory cortex also produced a lift and hold deficit however the force profiles were quite different. Although the placement of the fingers about the object was often grossly abnormal, there was no evidence of weakness and in some cases, the peak grip force and grip force rate significantly increased. The rate of force application was frequently discontinuous as if the animals were probing the contact surface for the appropriate sensory feedback. In spite of adequate grip force and successful lifting, the object was invariably dropped before the required time had elapsed. Using tactile cues alone the monkeys were unable to grasp small bits of food, although independent finger movements were clearly present. With additional visual feedback, the performance was somewhat improved, but still appeared clumsy when compared to the other hand. Funded by MRC of Canada.

\section{I-09}

\section{Nonlinear Dynamics of Intracranial EEG in Epilepsia Partialis Continua}

Richard Wennberg, Jose L. Perez Velazquez, Andres Lozano, Peter L. Carlen (Toronto, Ontario)

Background: A patient with a 37 year history of epilepsia partialis continua (EPC) of the right hand and secondarily generalized seizures was investigated with intracranial EEG. The complexity of the "interictal" (EPC) state was compared with the preictal and ictal dynamics of secondary generalization.

Methods: A 64 electrode subdural grid recorded from the left frontoparietal convexity. EEG time series complexity was analyzed by means of time delay maps and estimation of correlation dimension (CD) for electrodes overlying and adjacent to the epileptogenic area using INSITE software.

Results: Continous spiking between $2-4 \mathrm{~Hz}$ was recorded over the primary sensory hand region during the interictal and 
immediately preictal EPC state. A regional ictal onset as low amplitude fast activity marked the transition to secondary generalization. For the involved electrodes, $C D$ progressively dropped from $3.9 \pm 0.2$ to $2.8 \pm 0.1$ to $2.1 \pm 0.1$ in the interictal, preictal and ictal states respectively. Uninvolved electrodes $2 \mathrm{~cm}$. away showed a much higher complexity ( $C D>6.4)$ which did not change in the preictal state.

Conclusions: EPC can be described as a spatially restricted complex system of relatively low dimensionality. The intermittent transition from EPC to secondarily generalized seizure can be predicted prior to ictal onset by a localized decrease in the complexity of the system.

\section{I-10}

Posterior Hemi-hemispherectomy: A Valid Surgical Option in Certain Difficult Cases of Medically Intractable Epilepsy

O Vernet, JP Farmer, F Andermann, JG Villemure (Lausanne, Switzerland and Montreal, Quebec)

Introduction: For a limited number of patients with medically intractable epilepsy secondary to extensive posterior cerebral lesions and where hemispherectomy is contra-indicated, there still remains the option to perform a multilobar resection (or disconnection). We report our experience with posterior hemihemispherectomy (or temporo-parieto-occipital excision).

Patients: 7 patients $(2 \mathrm{~F} / 5 \mathrm{M})$ with intractable epilepsy were assessed with serial neurological examinations, EEG's, CT and/or MRI. Seizure onset ranged from 1 to 22 years of age (mean \pm SD: $8 \pm 8$ years). These patients exhibited extensive radiological lesions and electrical abnormalities involving the temporo-parieto-occipital region. They all presented homonymous hemianopsia and variable degrees of hemiparesis.

Results: At a mean age of 26 years (range: 17 to 42 years), every patient underwent a temporo-parieto-occipital resection or disconnection. Pathological diagnosis was Sturge-Weber in 1 case and sequellae of ischemic insult in 6 cases. Neither postoperative morbidity/mortality nor aggravation of the neurological deficits was deplored. In terms of epilepsy control, at a mean follow-up of 5 years: 5 patients were seizure-free and 2 had a $80 \%$ improvement rate of their seizure frequency.

Conclusions: Posterior hemi-hemispherectomy is a valid surgical option for cases of medically intractable epilepsy secondary to extensive unilateral multilobar lesions sparing the anterior hemisphere. This surgical technique carries an acceptable complication rate and provides excellent results in terms of epilepsy control.

\section{I-11}

Pathophysiological Mechanisms Of Limbic Seizure Evolution

L.F. Quesney, A. Olivier (Montreal, Quebec), F. Arruda (Riberrâo Preto, Brazil), R. Wennberg (Toronto, Ontario)

Goals: The pathophysiology of initial seizure propagation was investigated in 18 patients with temporal lobe epilepsy investigated with stereotactic depth electrodes.

Methods: All patients had three depth electrodes inserted orthogonally through the second temporal gyrus aimed at the amygdala (AM) anterior hippocampus (H) and parahippocampal gyrus $(\mathrm{PH})$, respectively. Chronic epidural electrodes along $\mathrm{TI}$ were available in most patients. The EEG onset and evolution was studied by visual inspection in 389 mesial temporal lobe seizures. Focal onsets were recorded from two or fewer contacts of one electrode and regional onsets from two or more contacts of one or more adjacent electrodes.

Results: 1) Size of the ictal generator: focal seizure onset ( $129 \mathrm{Sz}=33 \%$ ) involving $\mathrm{AM}, \mathrm{H}$ or PH gyrus was associated with a predominant ipsilateral intra-limbic seizure spread $(+/$ $70 \%)$. Regional mesial temporal lobe seizures $(260 \mathrm{Sz}=67 \%$ ) propagated mainly to ipsilateral temporal neocortex $(68 \%)$ or to contralateral limbic structures (30\%).

2) Modulation of seizure spread: regional seizures were most likely to spread if $\mathrm{PH}$ was involved at onset. Only $28 \%$ of $\mathrm{H}$ focal seizures spread beyond this structure.

Conclusion: Focal limbic seizures undergo a preferential intra-limbic propagation. Regional limbic seizures show a widespread seizure propagation suggesting that the availability of propagation pathways is proportional to the size of the ictal generator.

According to our findings two moderators of limbic seizure spread are proposed: the hippocampus exerts an inhibitory role for seizure spread whereas the parahippocampal gyrus facilitates extra-limbic propagation.

\section{J. FUNCTIONAL NEUROSURGERY}

\section{$\mathrm{J}-01$}

Exposure of Dopaminergic Cells to DGNF Prior to Transplantation Enhances Graft Survival and Sensorimotor Behavioural Recovery

\section{Mehta (Halifax, Nova Scotia)}

Although currently there are a number of medical and surgical treatment options for Parkinson's disease (PD), none of these are curative. The success of fetal transplantation in both the parkinsonian rodent and primate model has led to clinical trials in parkinsonian patients. In the parkinsonian rodent model, fetal grafting has improved rotational bias, however graft derived benefit for more complex sensorimotor deficits like forelimb akinesia has been limited. Demonstration of improvement in these tests is relevant as neural grafting attempts to clinically ameliorate motor symptoms.

We postulated that "priming" of fetal dopaminergic neurons in a hibernation media rich in glial derived neurotrophic factor (GDNF) prior to transplantation into rats with unilateral 6hydroxydopamine lesions may improve complex sensorimotor activity. Animals that received 400,000 cells exposed to GDNF demonstrated significant improvement of contralateral forelimb function and had improvement of rotational bias faster than animals that received cells not exposed to GDNF. Increasing the 
number of implanted cells to 800,000 also exposed to GDNF did not result in any significant increase in functional recovery.

As neural grafting procedures into the nervous system evolve and genetically engineered cells replace fetal tissue, crucial questions about cell number and trophic regulation will need to be addressed. This study demonstrates that the grafting of 400,000 cells exposed to GDNF prior to transplantation has a beneficial effect in the restoration of complex sensorimotor behaviour.

\section{J-02}

\section{An MRI-based Computerized System for the Surgical Treatment of Movement Disorders}

A.F. Sadikot, P. St-Jean, D. Clonda, R. Comeau, R. Kesrai, M. Panisset, G. Bertrand, T.M. Peters (Montreal, Quebec)

Introduction: Stereotactic functional neurosurgery requires excellent 3-dimensional (3-D) appreciation of relationships of stereotactic tools and deep brain structures. We have developed a computerized method that allows 3-D visualization of stereotactic atlases, tools and proposed lesions in the patient's stereotactic MRI space.

Methods: The cryogenic slice atlas of Shaltenbrand and Wahren was digitized in the horizontal plane and critical structures were identified. A volumetric atlas of the basal ganglia and thalamus was then created, using a spline-based deformation method. Stereotactic tools were modeled into the system, including a retractable loop-shaped leukotome, straight electrodes and curved electrodes. Virtual lesions generated by the leukotome were also modeled into the stereotactic space, allowing avoidance of critical structures such as the internal capsule and optic tract.

Results: The system was used for peri-operative interactive image-guidance with the OBT stereotactic instrument in 17 patients undergoing surgery for movement disorders. Non-linear deformation of the atlas was performed in order to integrate with the patient's stereotactic MRI. On physiological stimulation, close concordance $(<2 \mathrm{~mm})$ was generally found between anatomically modeled structures such as the internal capsule and sensory thalamus, and the position of the stimulator probe. Furthermore, postoperative MRI scans showed close spatial correlation between virtual lesions and actual lesions.

Conclusion: Computerized programs developed for frameless stereotaxis and non-linear registration of brain atlases to MRI scans can be applied in a novel manner to functional stereotactic neurosurgery. Interactive imaging methods allow reduction in exploratory trajectories and help prevent lesion impingement on critical structures, thus improving surgical results.

\section{J-03}

Validation of an Interactive Image Guidance System for Functional Neurosurgery

A.F. Sadikot, R.M. Comeau, B. Davey, M. Panisset, G. Bertrand, T.M. Peters (Montreal, Quebec)

Introduction: Functional neurosurgery for movement disor- ders requires excellent target accuracy. Appreciation of complex three-dimensional target sites would be enhanced by a system that reformats images in arbitrary oblique planes during preoperative planning and intraoperative stimulation or recording. We present a system for interactive image-guided planning, stimulation and lesioning based on co-registered CT, MRI or ventriculogram stereotactic data-sets.

Methods: We studied 20 patients who had stereotactic MRI, $\mathrm{CT}$ and ventriculography for thalamotomy(12) or medial pallidotomy(8) using the OBT instrument. Targets were determined with reference to the AC-PC line (Schaltenbrand-Warren Atlas) and verified in multiple planes on the MRI. Intraoperatively, stereotactic positions and trajectories of the stimulator, stimulation sites and leukotome were represented on the patient's own MRI allowing multiplanar verification in relation to critical structures.

Results: Comparison of MRI and ventriculogram-based target points revealed mean targeting errors of: anterior-posterior $1.7 \mathrm{~mm}(\mathrm{sd}=1.1)$; axial $1.6 \mathrm{~mm}(\mathrm{sd}=1.3)$; lateral $0.8 \mathrm{~mm}(\mathrm{sd}=1.1)$; mean error in 3D space $2.7 \mathrm{~mm}(\mathrm{sd}=1.0)$; mean AC-PC distance error $1.6 \mathrm{~mm}(\mathrm{sd}=1.4)$. All targets were adequate on post-operative MRI and no neurological complications were encountered.

Conclusions: Interactive image-guided stereotactic planning is valuable for surgical treatment of movement disorders. As MRI distortion and resolution limitations are overcome, MRIbased planning will emerge as a robust method for functional neurosurgery.

\section{J-04}

MRI \& PET Guidance In Surgery Of Epilepsy \& Brain Tumours Using The Viewing Wand System

A. Olivier, A.F. Sadikot, M. Cyr, R. Comeau, W. Boling, R Bittar, D. Reutens, D. Klein, B. Milner, T. Peters (Montreal, Quebec)

Over a period of five years we have carried out more than 500 procedures using the Allegro Viewing Wand System in the surgical treatment of epilepsy and intrinsic brain tumours.

In epilepsy, the frameless stereotactic MRI guidance has been applied to a variety of procedures such as transcortical selective amygdalohippocampectomy, callosotomy, resection of cortical dysplasia and implantation of intracranial depth and surface electrodes.

The main impact of this image guided surgery has been a reconsideration of the concept of cerebral localization. The crisp and accurate reconstruction of gyri and sulci has provided a quick and easy mean of identifying the topography of the central area and specifically of the central sulcus. For most cases of extratemporal surgery we now use routinely PET regional blood flow activation with $\mathrm{H} 215 \mathrm{O}$ and /or functional MRI for identification of sensori-motor zones of the hand, foot and tongue. For procedures on the dominant hemisphere we have relied more and more on speech activation studies which revealed with constancy speech activation centres to passive listening, word repetition and synonym generation. Speech activation have now been carried out for surgical planning in over 55 cases and sen- 
sorimotor activation over 50 patients. Comparative studies with functional MRI are ongoing.

The experience gained in the field of epilepsy has been readily applied to the resection of intrinsic brain tumours and other lesions located in the vicinity of speech centres and other high functional areas.

Examples will be provided to illustrate the rapid evolution of functional image guidance in neurosurgery.

\section{$\mathrm{J}-\mathbf{0 5}$}

\section{Effect of Ionizing Radiation on a Kindled Amygdalar Focus}

\section{K. Elisevich, K. Jenrow, A. Ratkewicz (Detroit, Michigan)}

Backgound: Empiric clinical evidence and experimental studies have shown ionizing radiation to alter focal epileptogenicity and to have an anti-epileptic effect in some situations. To characterize this influence, ionizing radiation was applied to an amygdala-kindled focus in the rat.

Methods: The right and left basolateral amygdala and right frontal cortex were implanted with concentric bipolar electrodes and rats were then kindled by electrostimulation at an af terdischarge threshold of the left amygdala through a series of 4 or a minimum of 13 stage 5 seizures. Seizure thresholds were determined before and at weekly intervals after irradiation. Beam collimation of a $60 \mathrm{Co}$ source $(1.25 \mathrm{MeV})$ or $\mathrm{x}$-ray (18 MV; peak photon energy) resulted in a $90 \%$ isodose line irradiation within a $2.5 \mathrm{~mm}$ radius of the electrode axis. Cohorts received a 10,18 or 25 Gy dose with controls remaining unirradiated.

Results: No significant changes in seizure thresholds were observed during a six month postirradiation period. Seizure character however was altered and frequently manifested as random double convulsive episodes upon stimulation in those animals undergoing greater numbers of stage 5 events with higher radiation doses applied. These were characterized by an initial stimulation-induced convulsion (80-200s), postictal quiescence (60-90s) and a second (spontaneous) ictus (15-150s). Running fits manifested only in the irradiated groups moreso at 25 Gy doses and appeared identical to those seen in other models. These were induced commonly with the initial threshold stimulation and also appeared randomly during the period of study.

Conclusions: Exposure to ionizing radiation to a maximum of $25 \mathrm{~Gy}$ does not affect the seizure threshold but does enhance excitability in a dose-dependent fashion (18 and $25 \mathrm{~Gy}$ ) after seizure initiation. Persistence of this excitability suggests that an alteration of the extracellular milieu has occurred possibly coupled with specific cell susceptibilities and changes in certain local circuit mechanisms.

\section{J-06}

\section{Motor Hand Activation Is Localized In Paul Broca's Pli De Passage Moyen}

W. Boling, A. Olivier, R.G. Bittar, D. Reutens (Montreal, Quebec)

Background: The ability to localize hand motor function on the surface of the brain would be an important tool for neurosurgeons in surgical planning.

Methods: All of the positron emission tomography (PET) activation studies for hand motor function performed at the Montreal Neurological Institute in the last three years were reviewed in patients without a mass or abnormality seen on the MRI. Each patient performed a self-paced hand opening and closing motor task. Using a computer assisted 3D reconstruction of the surface of each hemisphere studied, the hand motor area was identified in relation to cortical surface landmarks.

Results: The region of PET hand motor activation can be identified reliably on the surface of the brain by utilizing anatomical relationships to nearby structures. After identification of the precentral sulcus, the superior and middle frontal gyrus can be seen to abut the precentral gyrus at a right angle. A bend or genu in the precentral gyrus also is constantly seen between the superior and the middle frontal gyrus which points posteriorly (posteriorly convex). The location of hand motor activation is within the central sulcus at the apex of this posteriorly pointing genu.

Conclusion: The apex of the posteriorly pointing genu of the precentral gyrus leads to a deep cortical fold connecting the pre and postcentral gyrus. This deep fold was first described by Paul Broca as the pli de passage fronto-pariétal moyen and here lies the location of motor hand function. The correct location of PET hand motor activation could be reliably identified on the surface of the brain using these anatomical landmarks by physicians blinded to the PET activation results.

\section{K. GENERAL NEUROSURGERY}

\section{K-01}

Combined Extra-Intradural Approach For Ophthalmic Segment Aneurysms Of The Internal Carotid Artery

J. Demers, F. deSoultrait, M.W. Bojanowski (Montréal, Québec)

Background and purpose: Ophthalmic segment aneurysms are amongst the most difficult anterior circulation aneurysms to treat surgically. Reasons for this include problematic proximal control, intimate relationship of the optic nerve and difficult visualization of the proximal aneurysm neck. These are real skullbase aneurysms. The difficulty is emphasized by the fact that a lot of these aneurysms are now treated by endovascular methods. Skull-base surgery has facilitated the approach of ophthalmic segment aneurysms, particularly with exposition of the anterior portion of the cavernous sinus. We reviewed our experience with this approach in order to evaluate its safety and its indications.

Method: We used the combined extra-intradural approach for all surgically treated ophthalmic segment aneurysms since 1995 at our institution. A retrospective study of the cases was done.

Results: There were 2 male and 11 female patients $(n=13)$ with ages ranging from 21 to 67 years. A total of 14 aneurysms were treated (carotid-ophthalmic: 12, superior hypophyseal: 2). 5 patients had multiple aneurysms. Presentation included SAH $(n=6)$ and loss of visual acuity $(n=2) ; 6$ were discovered incidentally. In 5 cases endovascular treatment was tried and failed (large neck, 
GDC coil procidence). Fronto-temporal craniotomy was followed by the extradural and intradural steps in all patients. Postoperative angiogram showed complete aneurysm exclusion in 10/13 patients and minimal residual neck $(<1 \mathrm{~mm})$ in 3/13 patients. Evolution was favorable in all cases. One patient had partial third nerve palsy at short term follow-up. There was no mortality.

Conclusion: Combined extra-intradural approach of ophthalmic segment aneurysms permits better anatomical visualization and comprehension of the aneurysm and its neck. It minimizes optic nerve trauma by unroofing the optic canal. This approach allows safe obliteration of the aneurysm neck, including for cases in which the aneurysm presented with hemorrhage.

\section{K-02}

Endovascular Management of Basilar Bifurcation Aneurysms - Anatomical and Clinical Results

G Redekop, R Willinsky, K TerBrugge, W Montanera (Toronto, Ontario)

Background: Endovascular treatment with Guglielmi detachable coils (GDCs) is analternative technique for aneurysm obliteration when anatomical or patient factors preclude safe microsurgical clipping. We report our experience with 40 patients in whom GDC embolization of basilar bifurcation aneurysms was attempted.

Methods: All patients underwent superselective angiography and attempted embolization with GDCs. Angiographic and clinical results were prospectively recorded.

Results: Twenty-eight aneurysms presented with subarachnoid hemorrhage (SAH), 2 were symptomatic and 10 were incidental. Coils were placed in $30(75 \%)$ patients, while in 10 $(25 \%)$ coils could not be safely placed because of unfavorable anatomy. There were no permanent complications in seven patients who underwent embolization of incidental aneurysms. Of 23 patients in whom coils were placed after SAH, 20 were Grade 1 to 3 and 3 were grade 4 or 5 at the time of treatment. At last follow-up (mean 12.5 months) twenty-one $(91 \%)$ had a good outcome. Twenty patients underwent angiography at least 6 months after treatment (mean, 11 months). Complete occlusion was demonstrated in $13(57 \%)$, while $7(30 \%)$ had small neck remnants and $3(13 \%)$ had aneurysm regrowth requiring further intervention. No aneurysm bled after treatment.

Conclusions: Endovascular treatment of basilar bifurcationaneurysms is a safe and effective means of preventing aneurysm rebleeding. Long-term follow-up is required to determine the outcome of partially and completely coiled aneurysms.

\section{K-03}

Radiosurgery for Cavernous Angiomas of the Brainstem: Long-term Follow-up

A.F. Sadikot, M.A. Alonso-Vanegas, L. Souhami, A. Olivier (Montreal, Quebec)

Background: The surgical management of angiographically occult vascular malformations of the brainstem remains controversial. In our initial radiosurgery experience $(1987-1990)$ we treated ten patients with brainstem cavernous angiomas using the linear accelerator-based McGill dynamic radiosurgery technique.

Methods: Patients presented with brainstem hemorrhage, had lesions documented on MRI, and were judged to carry significant potential for surgical morbidity. The lesions ranged in maximum diameter from $6 \mathrm{~mm}$ to $25 \mathrm{~mm}$ (mean: $13.4 \mathrm{~mm}$ ). The maximum central dose ranged from 25 to 55 Gray (mean: 44 $\mathrm{Gy}$ ), and the minimum dose delivered to the margin of the cavernous angioma ranged from 5 to $20 \mathrm{Gray}$ (mean: $10 \mathrm{~Gy}$ ). Patients were followed at regular intervals, including clinical and MRI follow-up at 6 years after treatment.

Results: Most lesions were remarkably unchanged on longterm follow-up MRI, although cases of volume reduction of up to $20 \%$ were noted. In no case was there morbidity from radiation-related edema or necrosis. Furthermore, there have thus far been no instances of hemorrhage.

Conclusions: Our series provides evidence for possible protection from hemorrhage. However, in view of the low hemorrhage rate cavernous angiomas, a larger series of patients with long-term follow-up is required prior to drawing definitive conclusions. Other series suggest relatively high morbidity from radiation necrosis and edema after radiosurgery of brainstem cavernous angiomas. In our series, we emphasized attention to the 3 dimensional relationship of the lesion and surrounding brainstem, multiaxial dose planning, and a conservative marginal dose. Selected cases of compact inoperable cavernous angiomas of the brainstem can be treated with radiosurgery with minimum long-term radiation-related morbidity.

\section{K-04}

The Role of Nuclear Medicine Shuntograms in the Evaluation of Patients with VP Shunt Malfunction

BS Jhawar, DS Steven, R Sahjpaul, RF Del Maestro (London, Ontario)

Background: Acute VP shunt malfunction in children with congenital hydrocephalus is a common problem that is often difficult to diagnose. Work-up usually centres around clinical examination and CT imaging. Although Nuclear Medicine Shuntograms (NMS) are used in some centres, their role in the management of such patients remains uncertain.

Methods: Two reviewers retrospectively analyzed the charts of 75 children (age $<16 \mathrm{yrs}$ ) who presented to the emergency department with signs and symptoms consistent with shunt malfunction. Fifty-four patients met the following entry criteria: VP shunt (inserted $<1$ yr), Pudenz or Hakim type, simple shunts (i.e. no Y-connectors, no multiple shunts) and shuntogram within 24 hours of presentation. Gold standard comparisons were based upon operative findings, clinical course (6 wks) and CT scans, blinded to the initial shuntogram result.

Results: Preliminary data revealed the following test parameters and 95\% confidence intervals: Proximal end (sensitivity $=$ $.071(0.59,0.83)$, specificity $=0.93(0.87,1.0)$, positive-predictive value $=0.90(0.81,0.98)$, negative-predictive value $=0.80$ $(0.69,0.91)$, Distal end (sensitivity $=0.83(0.73,0.93)$, specifici$\mathrm{ty}=.079(0.68,0.90)$, positive-predictive value $=0.83(0.73$, $0.93)$, negative-predictive value $=0.79(0.68,0.90))$. 
Conclusions: Shuntograms have the ability to potentially localize the site of obstruction. This information can lead to a tailored operative procedure therefore minimizing costs potential morbidity. This study supports the excellent safety profile of this test and its ability to provide clinically useful information.

\section{K-05}

\section{Change in Ventricular Size and Effect of Ventricular} Catheter Placement on Shunted Hydrocephalus

SK Tuli, B O'Hayon, JM Drake (Toronto, Ontario), JRW Kestle (Vancouver, British Columbia)

Background: The pediatric cererbrospinal fluid (CSF) shunt valve design compared a standard valve to siphon-reducing valve (PS Medical Delta) and a flow limiting valve (Cordis OrbisSigma) and found no difference in CSF shunt failure (over all $40 \%$ at one year). However, the valves might be expected to have different effects on ventricular size, and the catheter position or environment to effect shunt failure. We therefore analyzed the ventricular size, as well as ventricular catheter environment in these patients.

Method: Ventricular size was measured at up to six different time intervals, using a modified Evans ratio (with incorporation of the frontal and occipital dimensions) in 344 patients. Ventricular catheter location was defined as occipital, frontal, atrial, or third ventricular and the tip as surrounded by brain parenchyma, touching brain or surrounded by CSF. Repeat measures ANOVA for unbalanced data was used to analyze ventricular size. A Cox model (with incorporation of time dependent covariates) was used to evaluate the contribution of etiology, shunt, ventricular size, ventricular catheter location and site amongst the 144 shunt malfunctions.

Results: Ventricular volume decreased in an exponential fashion over time and was coincident and parallel for the three valves $(p=0.8)$. Frontal and occipital ventricular catheter tip location had reduced Hazard Ratios (HR= $0.65,0.47$ respectively $\mathrm{p}=0.04$ ) as did ventricular catheters surrounded by CSF, or touching the brain ( $H R=0.21,0.34$ respectively $(p=0.0001)$. Larger ventricular size was predictive of subsequent malfunction (HR 2.27, $\mathrm{p}=0.027$ ). Myelominingocele (HR 1.81, $\mathrm{p}=0.002$ ) was also a contributor of shunt malfunction. Shunt valve design was not significant.

Conclusion: Ventricular catheter tip location and site in addition to etiology of hydrocephalus and ventricular size are significant contributors for CSF shunt malfunction. Decline of ventricular size over time is not affected by shunt valve design.

\section{CHILD NEUROLOGY}

\section{L-01}

\section{Very Prolonged Neonatal Seizures Do Not Cause Brain Damage}

E. Wirrell, E. Armstrong, J.Y. Yager (Saskatoon, Saskatchewan)
Background: Whether or not neonatal seizures result in neurologic injury is controversial. To clarify this issue a well defined model of prolonged status epilepticus (SE) was developed.

Methods: 10 day old rat pups, equivalent to a $36-40$ week gestation infant, were anesthetized with halothane, and silver wire electrodes stereotactically placed within the CAI region of the hippocampus of one hemisphere, and in the cortical motor strip of the opposite hemisphere. Electrocortical discharges and EKG were monitored. Following recovery for $2 \mathrm{hr}$, each pup was given a sc injection of $3 \mathrm{mg} / \mathrm{kg}$ of kainic acid (KA), followed by a $2 \mathrm{mg} / \mathrm{kg} / \mathrm{hr}$ sc continuous infusion for $3 \mathrm{hrs}$. Separate groups of rat pups, were allowed to recover to $14 \& 30$ postnatal days for neuropathologic assessment. In other groups, rat pups were sacrificed at times $0,1,2,3,4,5$, and 6 hrs post-injection for evaluation of blood glucose, lactate, and betahydroxybutyrate (B-OH).

Results: Overall mortality rate in seizuring animals was $21 \%$. All rat pups receiving KA injections displayed clinical seizures characterized by scratching, salvation, circling, shaking, and tonic posturing. Electrographically, all rat pups had continuous high voltage polyspike and sharp wave activity lasting a mean of $282 \mathrm{~min}$ (range 240-327 min). In surviving animals heart rate was unaffected through the duration of the seizure. At I hr postinjection, blood lactate rose dramatically to $5 \mathrm{X}$ control values, and subsequently declined to near normal. Blood glucose concentrations remained normal during the $1 \mathrm{st}$ hr post-injection. During the $2 \mathrm{nd} \mathrm{hr}$, glucose concentrations significantly declined from control of 5.03 to $2.34 \mathrm{mmol} / \mathrm{L}$. Glucose remained at this level until SE subsided, at which point it rapidly returned to normal. B-OH rose gradually over the period of status, and in correlation with a reduction of both glucose and lactate concentrations. With the discontinuation of seizures, B-OH returned to normal. Neuropathologic assessment with $\mathrm{H} \& \mathrm{E}$ staining revealed no brain damage in either the neocortex or brainstem structures.

Conclusions: Continuous electrographic and clinical seizure activity for $4 \mathrm{hrs} 42 \mathrm{~min}$ in the newborn rat does not cause brain damage. The newborn brain, while highly sensitive to seizure activity, is clearly resistant to its sequelae, possibly due to the immature brains ability to utilize alternate substrates as metabolic fuels. A re-examination as to the need for treatment of neonatal seizures seems reasonable.

\section{L-02}

Can Children With Benign Rolandic Epilepsy Be Managed With An Anticonvulsant At Bedtime Only?

\section{Robert Munn (Toronto, Ontario)}

Many children with benign rolandic epilepsy (BREC) do not require treatment. Some children with diurnal or frequent seizures may require an anticonvulsant. The purpose of this study was to determine if children with BREC and nocturnal seizures only can be managed with a single h.s. dose of medication.

Seventy-two children with BREC were evaluated between Jan. 96 and Dec. 97 . In 14 children my intention had been to treat at h.s. only. Three children were switched to BID dosing 
because of either diurnal seizures (2) or multiple nocturnal seizures unresponsive to single h.s. dosing (1). One child was on no medication at parents request.

Eight children (mean age epilepsy onset 6 years) were seizure free on carbamazepine (Tegretol chewtab - 4, CR formulation ${ }^{\circledR}$ - 4) with a dose range of $1.0-6.6 \mathrm{mg} / \mathrm{kg} /$ day (mean 4.3 $\mathrm{mg} / \mathrm{kg} /$ day). Pretreatment seizure frequency in these children was $2-5 /$ week in 3 and $1-4 /$ month in 5 . Another child showed significant improvement on $5.7 \mathrm{mg} / \mathrm{kg} / \mathrm{day}$ of carbamazepine. One child had minimal improvement with clobazam $14 \mathrm{mg} / \mathrm{kg} / \mathrm{day}$.

In conclusion, some children with BREC can be successfully managed on low dose h.s. only carbamazepine.

\section{L-03}

\section{Topiramate in Intractable Epilepsy - A Cautionary Note}

J.M. Dooley, P.R. Camfield, E. Smith (Halifax, Nova Scotia) P. Langevin (Laval, Quebec)

Background: We present our first 44 patients treated with Topiramate, a new antiepileptic drug (AED) which was introduced to Canada in 1997.

Results: All of the patients had intractable epilepsy. Six were on 1 other AED, 23 were on 2 other AEDs and 14 were on 3 or more concommitant AEDs. One patient had failed all other available AEDs and was on Topiramate monotherapy. The mean age at treatment initiation was 175.7 months $(\mathrm{SD}=81.3$ months, range 39-372 months). Of those who tolerated Topiramate, seizure reduction of $>90 \%$ was achieved in 3 patients $(7 \%), \geq$ $50 \%$ in 9 patients $(20.5 \%)$ and $<50 \%$ in a further 8 patients $(18 \%)$. A deterioration in seizure control was seen in 8 patients (18\%).

Adverse effects, sufficient to require topiramate discontinuation, were experienced by 23 patients $(52 \%)$. Sixteen families $(36 \%)$ complained of unacceptable cognitive dulling. All adverse effects resolved upon topiramate discontinuation. For those who discontinued therapy the mean duration of therapy was 2.5 months (range 0.25-8 months). The rate of dose escalation in those without adverse effects to Topiramate (mean 0.55 $\mathrm{mg} / \mathrm{Kg} /$ week, range $0.22-1.38 \mathrm{mg} / \mathrm{Kg} /$ week) was similar to that of patients who discontinued because of adverse effects (mean $0.52 \mathrm{mg} / \mathrm{Kg} /$ week, range $0.22-1.08 \mathrm{mg} / \mathrm{Kg} /$ week). Seventeen (39\%) patients have remained on topiramate.

Conclusions: Although Topiramate produced $a \geq 50 \%$ reduction in seizure frequency in $27 \%$ of this group of patients with intractable epilepsy, caution is required as cognitive impairment may limit its usefullness. The rate of dose increase was not correlated with the incidence of adverse effects.

\section{L-04}

\section{Surgery for catastrophic epilepsy (C.E.) in infants}

A. Turmel, L. Carmant, D.G. Human, A. Lortie, Y. Robitaille, R. Lambert, J.G. Villemure (Montreal, Quebec)

By definition the term catastrophic epilepsy (C.E.) is reserved for infants with severe seizures of early onset. Severity is judged by the frequency, intractability of the seizures and possible severe developmental delay.

Three infants (6 to 23 months) were treated in our pediatric epilepsy program at Sainte-Justine Hospital. All had C.E (one had early onset status epilepticus). All had developmental delays. Investigation consisted of scalp video EEG, MRI, SPECT studies, one had PET and another had invasive monitoring with subdural grids. Preoperatively $2 / 3$ were localized by scalp EEG, MRI, SPECT and PET, $1 / 3$ was localized by subdural grids only. Operatively $2 / 3$ had focal resections (frontal, occipitotemporal), $1 / 3$ had two procedures, one focal resection (occipital) and a functional hemispherotomy.

Results: $3 / 3$ are seizure free postoperatively (follow-up 12 to 18 months), $2 / 3$ have had significant developmental catch up one is severely developmentally delayed. $3 / 3$ had histological diagnoses of cortical dysplasias ranging from diffuse microdysgenesis to focal dyplasias with giant balloon cells.

Aggressive investigation and treatment of early onset C.E. is worthwhile. Longer follow-up is needed to evaluate future developmental milestones. These cases should be referred to specialized pediatric epilepsy centers.

\section{L-05}

\section{Benign Occipital Lobe Epilepsy with onset during Adolescence}

A Ogunyemi (St. John's, Newfoundland)

Background: In the last 10 years, the electroclinical features of the syndromes of childhood-onset occipital lobe epilepsy have been well delineated. The electroclinical characteristics and prognosis of a syndrome of adolescent-onset occipital lobe epilepsy are less familiar.

Methods: Among patients attending the seizure clinic at the Health Sciences Centre, St. John's, Newfoundland, we identified 4 patients diagnosed with occipital lobe epilepsy who have similar clinical and EEG findings.

Results: The four patients, all University students, were female. Seizures began between age 15 years and 17 years. The seizure manifestation consisted of generalized convulsion preceded by the sensation of twitching of the eyes, occurring within 30 minutes of arousal from sleep. Nocturnal seizures, visual hallucinations, nausea and vomiting were denied. Two patients suffered interictal attacks of headache. They were all seizure-free on low-dose antiepileptic monotherapy.

Interictal EEG in all of them showed: normal background rhythms and trains of bilateral rhythmic activities lasting 2 to 5 seconds in the posterior head regions, upon eye closure. Ictal EEG in one patient revealed train of bilateral occipital rhythmic activity for 5 to 6 seconds, succeeded by generalized attenuation and subsequent tonic-clonic movements.

Conclusion: Benign adolescent-onset occipital lobe epilepsy is characterized by (i) normal neurological findings, (ii) generalized convulsions during arousal from sleep and (iii) EEG showing transient trains of rhythmic activities in the posterior head regions upon eye closure. 
L-06

Post-Stroke Seizures in Childhood: Experience from the Canadian Pediatric Ischemic Stroke Registry

\section{B. Meaney, R. Curtis, G.A. deVeber (Toronto, Ontario)}

Background: Seizures following cerebral infarction are common. Little is known about incidence, risk factors and outcome of post-stroke seizures in childhood.

Methods: Children with arterial ischemic stroke $(\mathrm{AIS})(\mathrm{n}=243)$ and sinovenous thrombosis $(\mathrm{SVT})(\mathrm{n}=78)$ have been followed prospectively at 16 tertiary pediatric centers in Canada since January 1992. Data were collected regarding clinical and radiographic features of the stroke as well as number, type and timing of seizures.

Results: Seizures occurred in $36 \%$ of AIS and $50 \%$ of SVT patients. Onset of seizures was usually within 24 hours of stroke onset (AIS 89\%, SVT 95\%). Age less than 2 years was a risk factor for seizures in AIS (relative risk $=2.7, \mathrm{p}<0.001$ ) and SVT (relative risk $=2.2, p=0.001$ ). AIS involving large vessel distribution including anterior and middle cerebral arteries carried a 1.7 times greater risk of seizures compared to small vessel or vertebrobasilar territory infarcts $(p=0.002)$. Hemorrhage was associated with more seizures in SVT (relative risk $=1.6$, $\mathrm{p}<0.05$ ) but not AIS patients. Seizures at presentation tended to predict abnormal neurologic outcome in SVT patients (relative risk $=1.5$ ) but the trend was not statistically significant $(\mathrm{p}=0.15)$. Recurrent seizures ( $>4$ weeks post-infarct) were seen in $10 \%$ of AIS and $20 \%$ of SVT patients.

Conclusions: Seizures frequently complicate AIS and SVT in childhood, and may predict a worsened neurologic prognosis. Patients less than 2 years of age with SVT or with AIS involving large vessels are at highest risk of early seizures and should be considered for prophylactic administration of anticonvulsant medication. (Funded by Bloorview Children's Hospital Foundation)

\section{L-07}

\section{Sinus of Valsalva Aneurysm: A Rare Cause of Embolic} Stroke in Childhood

\section{D.Johnston, M.Sargent, A. Hill (Vancouver, British Columbia)}

Background: Aneurysms of the Sinus of Valsalva in the aortic root may be congenital, associated with Marfan's syndrome, infections of the aortic ring or post-aortic root replacement. Such aneurysms may be visualized by transthoracic echocardiography. Their natural history includes progressive enlargement and eventual rupture. There have been 2 reported cases of embolic stroke in adults associated with isolated aneurysms of the Sinus of Valsalva. However, it has not been reported in childhood.

Methods: We report embolic stroke associated with aneurysm of the Sinus of Valsalva in a 7 year old Caucasian girl.

Results: This girl presented with sudden collapse followed by dense right hemiplegia, global aphasia and decreased level of consciousness. Computed tomography demonstrated hyperdense left middle cerebral artery (MCA). Diffusion MRI scan demonstrated increased signal in the left hemisphere involving extensive territory of the MCA. Magnetic resonance angiography demonstrated proximal occlusion of the left MCA, normal carotid vessels and no evidence of other intracranial vessel abnormality. Echocardiogram revealed a saccular aneurysm of the right Sinus of Valsalva with mildly abnormal aortic valve motion.

Conclusions: Stroke is uncommon in childhood. Causes of embolic stroke in children are often elusive despite extensive investigation. In most instances, such investigation should include close examination of peri-aortic and supra-aortic regions by transthoracic echocardiography for the possible, albeit rare occurrence of a congenital Sinus of Valsalva aneurysm which may be a source of embolus and require treatment with anticoagulation and corrective surgery.

\section{L-08}

\section{Clinical Heterogeneity in a Kindred With Familial Hemiplegic Migraine with Cerebellar Atrophy}

\section{Keegan, N. Lowry (Saskatoon, Saskatchewan)}

Familial hemiplegic migraine (FHM) is a relatively rare entity which is sometimes associated with nystagmus and cerebellar atrophy. The gene for FHM with cerebellar atrophy has been mapped to chromosome 19p13 and codes for the $\mu-1$ subunit of a brain-specific $\mathrm{P} / \mathrm{Q}$-type calcium channel. This gene has also been implicated in episodic ataxia type 2 and spinocerebellar ataxia type 6.

The propositus is a twelve year old girl who presented with hemiplegic and vertebro-basilar migraines. The latter were associated with recurrent episodes of horizontal and vertical diplopia. When asymptomatic she had bilateral horizontal and upbeating gaze-evoked nystagmus. She had saccadic smooth pursuit eye movements and she failed to suppress the vestibulo-ocular reflex. Magnetic resonance imaging showed marked atrophy of the cerebellar vermis. She had a strong family history of hemiplegic migraine. The pedigree showed an autosomal dominant inheritance pattern.

The other affected family members showed variable age of onset, severity of hemiplegic episodes and age at resolution of symptoms. The hemiplegic symptoms ranged from minimal to severe symptoms that required days of hospitalization. The severity of symptoms did not correlate with the age at onset or age at resolution. In the eldest living affected relative (age 91), the episodes had resolved and there were no permanent sequelae. This may demonstrate the relatively benign outcome of this disorder even with repeated severe hemiplegic events. This kindred demonstrates the clinical heterogeneity of hemiplegic migraine with cerebellar atrophy and shows the eventual resolution of symptoms at various ages with lack of permanent disability despite repeated hemiplegic events. 


\section{L-09}

\section{Herpes Simplex Encephalitis in Childhood}

H Kolski, EL Ford-Jones, M Petric, S Richardson, S Nelson, D MacGregor (Toronto, Ontario)

Background: Herpes simplex virus (HSV) is the most common cause of sporadic fatal encephalitis in the Western World, with approximately $30 \%$ of cases occurring in children. We established a prospective registry to identify the incidence of HSV and other agents in acute encephalitis and analyze affected patients' clinical courses and outcomes.

Methods: Patients with HSV encephalitis were identified based on: 1) positive serology (4 fold rise between acute \& convalescent titres) and 2) CSF PCR.

Results: Five of 76 (6.6\%) children with encephalitis (Jan 94Dec 96) were diagnosed with HSV infection. All 5 patients had positive antibody responses and 3 of 5 had confirmatory CSF findings by PCR ( 2 with HSV 1 and 1 with HSV 2). There were 4 males and 1 female, ages 2-33 months. Presenting clinical features included fever (5), partial seizures (5), focal neurological signs (2), decreased level of consciousness (1). All cases demonstrated a CSF pleocytosis (16-64 WBC/uL), as well as abnormal EEG and neuroimaging findings (often multifocal). Despite recommended treatment with acyclovir, all 5 patients demonstrated significant neurological impairment at discharge including combinations of homonymous hemianopsia (3), oromotor dysfunction (2), altered muscle tone (2), hemiplegia (1) and breakthrough seizures (1). One patient was readmitted 11 days following discharge with an apparent relapse.

Conclusions: The spectrum of HSV encephalitis in paediatric patients includes multifocal involvement of the brain, in contrast to the temporal lobe predilection commonly seen in adults. HSV encephalitis continues to cause substantial morbidity in children despite rapid diagnostic methods (PCR) and availability of acyclovir.

\section{L-10}

\section{Leptomeningeal Spread of Primary Brain Tumors, an Unusual Complication in Children}

D. J. Buckley, L. F. Jardine, J. P. Hand, C. Pushpanathan, F. Maroun (St. John's, Newfoundland)

Objectives: To report two boys who developed carcinomatous meningitis secondary to primary brain tumors.

Background: Leptomeningeal spread of primary brain tumors is uncommon in the pediatric age group. Recognition of this possibility and diagnosis is not easy. Even with the use of mod- ern technology it often remains a clinical diagnosis with confirmation at post-mortem.

Results: A six-year-old boy presented with a three-month history of headache and vomiting. Examination revealed focal neurological signs. Imaging confirmed a large intraventricular mass lesion, which was resected. This recurred and spread despite repeat surgery, chemotherapy and radiotherapy. He developed back pain and cranial nerve palsies.

The second case is an eleven-year-old boy who presented with partial complex seizures. Investigations revealed a calcified mass in his right temporal lobe. This was resected and defined as a low-grade glioma. The tumor regrew and repeat resection revealed a high-grade glioma. He received chemotherapy and radiotherapy. Some months later he developed back pain.

Conclusions: Carcinomatous meningitis invariably leads to a fatal outcome. Early diagnosis and management is difficult. It is important to be aware of this complication of CNS malignancies even in the pediatric age group.

\section{L-11}

\section{Canadian Pediatric Neurologists Involvement in Ukraine}

V. Martyniuk (Kiev, Ukraine), E. Gauk (Edmonton, Alberta)

In 1992, the Canadian Government, through the Canadian International Development Agency (CIDA) funded an aid program called Medical Project OSIVTA, a teaching project which focused on the management of diseases of mothers and children who had been affected by the Chernobyl disaster. In the six years that followed 98 Canadian medical educators from every medical school in Canada volunteered to spend two or more weeks in Ukraine for a total of 173 teaching missions. Of this group 13 were pediatric neurologists who went on 21 teaching missions and lectured at 10 major medical centers in Ukraine. In the same period of time, 73 Ukrainian maternal and child health specialists trained in Canada, each for a period of four months. Of these five were pediatric neurologists who now work in four major medical centers in Ukraine. The impact of the Canadian contribution has been observed in the following areas: 1) Use of investigations and medications formerly not available in Ukraine. 2) Decreased used of unproven therapies and medications. 3) Decreased hospitalization of children for minor neurological ailments. 4) Encouraged the development of neurological co-workers such as speech therapists and physiotherapists. 5) Increased immunization of children which were earlier denied immunization for a host of suspected neurological problems. 6) Improved communication with "Western" medicine after a prolonged period of isolation. Medical Project OSVITA continues with University of Alberta and donor support. 


\section{Poster Presentations}

\section{O. HISTORY}

\section{0-01}

\section{Medical Books in the Library of Leonardo da Vinci}

\section{RF Del Maestro (London, Ontario)}

Background: In 1967 two Leonardo notebooks now known as Madrid Codex I and II were found in the Bibliotheca Nactional of Madrid. On Folio 2 verso and 3 recto of Codex II is a document written from right to left in Leonardo's typical mirror script listing the books present in Leonardo's Library. One hundred and sixteen items are listed which outline the wide range of Leonardo's interests. The purpose of this presentation is to outline the influence of the medical books present in Leonardo's personal library and the medical books that he is known to have consulted on his anatomical and physiological thought related to the central nervous system (CNS).

Methods: Leonardo's notebooks were carefully scrutinized to find the medical and health related books which influenced his investigations of the CNS.

Results: Fourteen of the 116 items listed by Leonardo in Codex II relate directly to the medical world in which he lived. Leonardo also refers to Galen's "De Utilita" (On the Usefulness of the Parts of the Body) and frequently refers to Avicenna. Leonardo derived CNS anatomical information from Mondino De'Luzzi's "Anothomia" published in Johannes De Ketham's "Fassciculu medicine - latino", Alexander Beneditti's Anatomia ("Libro di notomia in casa al Munistero"), and Guy de Chauliac's Surgery ("Guidone in cerusia")

Information related to ocular anatomy and function was obtained from John Pecham "prospettiva commune" while Albertus Magnus ("filosofia d'Alberto Magno") was basic to his understanding of the function of the ventricular system. His concepts of brain function were also influenced by Greek and Roman authors distilled through the writings of Pliny, Livy and Avicenna. Interestingly, Leonardo owned a book on the anatomy of the horse, a number of dietary or health related books and a book on the medical assessment of urine.

Conclusions: Leonardo's understanding of the CNS was heavily influenced by the medical books present in his personal library or known to him and these were the basis on which he formulated his anatomical and physiological studies of the CNS.

\section{0-02}

\section{Dr. H.H. Hepburn and the Development of Cervical Spine} Traction at the University of Alberta

\section{I.F. Parney, P.B.R. Allan, K.C. Petruk (Edmonton, Alberta)}

Cranial traction to reduce cervical spine fractures has been credited primarily to Dr. W.G. Crutchfield and Dr. C.C. Cole of the Medical College of Virginia. In 1933, they reported insertion of extension tongs into the calivarium of a 23 -year-old woman with a traumatic C2-C3 fracture. Unaware of Crutchfield's work, Dr. K.G. McKenzie of the University of Toronto also reported application of ordinary ice tongs to the skull for cervical traction in 1935. However, cervical traction skull tongs developed by Dr. H.H. Hepburn of the University of Alberta may predate both reports.

Dr. Hepburn was born in Manitoba in 1885. He received medical training at McGill University prior to being appointed as a surgeon in the Royal Army Medical Corps during the first world war. In 1920, he set up practice at the University of Alberta as Edmonton's first neurosurgeon. At some point between 1920 and 1930, Dr. Hepburn constructed and began routine application of the world's first pair of cervical traction skull tongs.

Unfortunately, Dr. Hepburn never published a report concerning the development of cervical spine traction. However, Crutchfield's 1933 report describes the traction device as the "Edmonton tongs." This strongly suggests an awareness of Hepburn's work. Although the tongs have been exhibited at the Smithsonian Institute, Dr. Hepburn's contributions have not been widely acknowledged. Dr. Hepburn was an unsung pioneer in cervical spine traction and deserves wider recognition.

\section{0-03}

\section{The Origin of the Drake Fenestrated Aneurysm Clip}

CG Drake, RF Del Maestro (London, Ontario)

Background: Early in 1969 two patients with large basilar bifurcation aneurysms were admitted to the neurosurgical unit at Victoria Hospital in London, Ontario. At that time to clip an upward projecting bifurcation aneurysm it was essential to separate the PI segment of the posterior cerebral artery from any adherence to the side of the aneurysm neck and the perforators to place the clip. In both cases the ipsilateral PI segment was densely adherent to the transparently thin neck and waist of the aneurysms and could not be dissected free. The aneurysms were packed, the patients post-operative cases were uneventful but both patients subsequently rehemorrhaged and died.

In September 1969 another similar case presented to the service. In brooding over the problem, C.G.D. came to the realization that PI need not be dissected free if an opening could be made at the take off of the clip blades from their handle wherein Pl could be accommodated while the blade lengths closed over the neck of the aneurysm. A number of clips were ruined trying to fashion a suitable aperture. C.G.D.called Dr. Mayfield in Cincinnati on a Friday and his engineer, George Kees, created a small aperture at the origin of the clip blades based on careful aneurysm measurements. Dr. Mayfield and Mr. Kees spent the weekend working on the problem and three beautifully fenestrated clips arrived by express mail. The patient's aneurysm was obliterated completely with PI intact within the fenestration.

It was soon discovered that many other intracranial structures could be safely preserved within the fenestration. A few years later, C.G.D.was able to clip a giant aneurysm using the fenestrated clips in tandem, largely solving the problem of many giant aneurysms whose necks were soft enough to be collapsed. 


\section{0-04}

The Contribution of W.D. Stevenson to the Development of Neurosurgery in Atlantic Canada

\section{K. Mukhida, I. Mendez (Halifax, Nova Scotia)}

The establishment of a neurosurgical department in Halifax in January 1948 was the first such service in Atlantic Canada. Its development occurred at both a receptive place and time. The Victoria General Hospital was in a period of growth and medical specialization and departmentalization, in part inspired by the Flexner Report of 1910. Atlantic Canadians were also increasingly looking to specialists and hospitals for medical care.

Although this social environment encouraged the establishment of surgical specialty services, the development of neurosurgery in Halifax, as in other parts of Canada, was closely linked to the efforts of individual neurosurgeons. It was only in 1924 that the first neurosurgical service was created in Canada; K.G. McKenzie was the country's first neurosurgeon, and his program in Toronto became one of the chief training centres in the country. After training with McKenzie, W.D. Stevenson was recruited to establish the first neurosurgical service in Atlantic Canada. From the outset and over his twenty-six years as Department Head at the Victoria General Hospital and Dalhousie University, Stevenson worked to maintain the department's commitment to clinical practice, medical education, and research. He immediately assumed the role general surgeons previously had in the management of patients with central nervous system injuries, and assumed responsibility for cases that had previously been referred to the Montreal Neurological Institute and the Lahey Clinic in Boston. Although Stevenson single-handedly ran the service for several years after its inception, by the time of his retirement he had helped it to grow to include five attending staff surgeons and perform over two thousand procedures each year. The history of the development of neurosurgery in Atlantic Canada can therefore be viewed in a historiography that maintains the importance of Stevenson's contributions to the development of the service by placing them in the context of the social environment of Atlantic Canada.

\section{O-05}

\section{Pediatric Neurosciences at the University of Western Ontario}

R.F. Del Maestro (London, Ontario)

Background: When the University of Western Ontario was established in 1881, pediatric patients were under the care of the Division of Obstetrics and Diseases of Women and Children. Pediatrics was separated from medicine in 1920 and Dr. W.J. Tillman was named the first Professor of Pediatrics. Dr. W.L. Denny, the first physician in London, limiting his practice only to pediatrics, began his practice in 1920. War Memorial Children's Hospital opened in 1922 and up until its closure in 1983, was the focus for pediatrics in Southwestern Ontario. During this early period pediatric patients with neurological problems requiring specialized surgical or medical care were frequently transferred to other centres. In November of $1951 \mathrm{Dr}$. Charles Drake returned to the University of Western Ontario, initiating a new era for both adult and pediatric neurosurgery patients in Southwestern Ontario. In 1961, Dr. George Hinton opened his practice focussed on neurological diseases in childhood. Dr. Loren Amacher joined the Division of Neurosurgery in 1970 devoting the majority of his practice to pediatric neurosurgery. These individuals contributed significantly to the development of Pediatric Neurosciences at the University of Western Ontario. The objective of this presentation is to outline the evolution of Pediatric Neurosciences at the University of Western Ontario focussing on pediatric neurosurgery and neurology from 1951 to the present.

\section{0-06}

Edward William Archibald (1872-1945): Father of Canadian Neurosurgery

\section{A. Turmel (Montréal, Québec)}

In March 1901, James Bell, a Montreal surgeon under the guidance and clinical skills of J. Stewart ("neurologist") at the Royal Victoria Hospital (RVH) proceeded to resect a subcortical glioma from the frontal lobe of a patient. This would be the first successful attempt at "cerebral localisation based" neurosurgery in Canada. E. Archibald who was responsible for surgical pathology did the histological diagnosis.

Soon after this ground breaking event, Archibald demonstrated increased interest in the surgery of the CNS and was sent by Bell (1906) to Queen's Square to study under Horsley and Gowers. On his return (1907) he practiced interventions on the N.S., published his results in local journals, but most of all he published a monograph of 385 pages. "Surgical affections and wounds of the head" in Bryant and Buck's "American Practice of Surgery". This same year 1908 H. Cushing would also publish his first book "Surgery of the head" in Keen's "Surgery: its principles and practice".

After Bell's death, Archibald assumed all neurosurgical activities at the RVH. He would become the first Canadian member of the first North American Neurosurgical Society "The Society of Neurological Surgeons" in 1920. Side by side with H. Cushing, C. Frazier, C. Elsberg and many others.

At the same time $\mathrm{K}$. McKenzie who would become the first carreer neurosurgeon in Canada had not even begun his training with $\mathrm{H}$. Cushing.

During the twenties, Archibald's interest waned from neurosurgery towards thoracic surgery but his 20 years of active interest in the surgery of the CNS did not cease and he was reponsible for the coming of Wilder Penfield to Montreal and in doing so assured the continued neurosurgical activities at the RVH (1927) and then MNI (1934).

In fact E.W. Archibald deserves to be recognized as the father of Canadian Neurosurgery. 
0-07

\section{Fedor Krause: Pioneer Seizure Surgeon}

\section{R Leblanc (Montreal, Quebec)}

Fedor Krause systematically performed cortical resection for treatment of focal epilepsy and, based on the results of cortical stimulation, produced an accurate functional map of the human motor strip. He estimated the incidence of epilepsy in Prussia at $1 / 1,000$ and advocated early surgery when medical management had proved ineffective. He operated under chloroform anesthesia, which was allowed to wear off for cortical stimulation that he used for mapping the motor strip and identifying the epileptic focus, the "primary spasming centre," before resecting it. Results of stimulation were recorded by three observers for later analysis. Histologic examination of resected specimens, performed by Brodman, confirmed the precentral origin of motor responses and identified the leptomeningeal-cortical scars that Penfield would term the meningocerebral cicatrix. Krause operated on 55 patients (1893-1912) and obtained follow-up data on 33 by detailed mailed questionnaires. There were four "cures" (seizure-free at 5 years), 8 "marked improvements," and 3 "very good results; 11 patients were "unchanged," 3 were "aggravated," and 4 died of status epilepticus. Krause helped establish the structure-function relationship of the human precentral gyrus and demonstrated that resection of a cortical epileptic area is feasible for treatment of medically intractable seizures.

\section{O-08}

\section{Penfield's Contribution to Cerebrovascular Physiology - An Epistemological Study}

\section{R Leblanc (Montreal, Quebec)}

Penfield's early study of brain wounds (Penfield, 1924; RioHortega and Penfield, 1927) and examination of tissue resected in the treatment of post-traumatic epilepsy (Foerster \& Penfield, 1930) focused attention on the incorporation of leptomeningeal and intracerebral vessels within cerebral scars. This led Penfield to hypothesize a local intracerebral vasomotor reflex producing epileptogenesis and post-ictal ischemia and atrophy from recurrent vasoconstriction $(1930,1932)$. The investigation of this hypothesis led to the identification of intracerebral vascular nerves (Penfield, 1932); to the discovery of an intracranial parasympathetic vasodilator system originating in the medulla oblongata (Chorobski and Penfield, 1932); to a study of the effects of sympathectomy on experimental epilepsy (Gage, 1931); to an extensive study of the innervation of intracranial blood vessels and dural sinuses (McNaughton, 1938); to the first study of middle cerebral artery occlusion in monkeys; and to the first study, by Echlin (1938), demonstrating that local prolonged vasoconstriction - he termed it "vasospasm" - produces histological ischemic changes in the brain. Later studies from Penfield's laboratory would stress the importance of cerebral vasospasm and attempt its pharmacological reversal (with Lends and Rasmussen, 1960, 1961). His interest in the circulation of the epileptic brain stimulated study of the microcirculation in man in various disease states, most notably cerebral arteriovenous malformations. These observations demonstrate that Penfield's scientific method relied on novel observations permitted by new technological advances (silver and gold stains) leading to the formation of hypotheses subsequently investigated in the laboratory, in turn producing new knowledge expanding his area of interest; and provide epistemological insight into the scientific method as applied to the study of the nervous system in the early part of the 20 th century.

\section{0-09}

\section{Cortical Resection for Epilepsy Before Penfield and Jasper}

\section{R. Leblanc (Montreal, Quebec)}

Cortical resection for the treatment of medically intractable epilepsy is a subject of renewed interest. The first such operation was performed in 1886 by Sir Victor Horsley who removed a cerebral scar and its associated brain tissue to treat a case of post-traumatic Jacksonian epilepsy under the watchful eye of Hughlings Jackson. This type of operation was then abandoned because of difficulties in localizing the motor strip by means of external landmarks to guide cortical resection. It was taken up again by Fedor Krause who operated on 54 patients with Jacksonian seizures between 1893 and 1910. Krause identified the motor strip by faradic stimulation of the brain and resected the cortical area corresponding to the site of origin of the seizure, which he called the "primary spasmic centre." Using the data obtained from cortical stimulation during the course of these operations, he produced one of the first functional maps of the human motor strip. Otfrid Foerster in 1924, operating on German World War I veterans with posttraumatic epilepsy, was the first to use forced hyperventilation to bring on a seizure and in 1925 he was the first to use ventriculography to preoperatively identify and localize the area of cerebral atrophy produced by a cerebral cicatrix. Confirmation of the epileptogenicity of the proposed area of resection was achieved intraoperatively under local anesthesia by reproducing the clinical seizure using either direct traction on the meningocerebral cicatrix or faradic stimulation of the suspected epileptogenic area. In 1934 Foerster would be the first to localize a seizure focus by intraoperative electrocorticography. Penfield's study of Foerster's surgical specimens in 1928 led the two men to formulate the first testable hypothesis of the etiology of post-traumatic epilepsy, which held that the seizures were produced by a focal "vascular reflex" resulting from traction on arteries enmeshed within a contracting meningocerebral cicatrix. Penfield's investigation on this hypothesis led to the first demonstration of the innervation of cerebral blood vessels in 1932 and produced the first study of cerebral vasospasm by Echlin in 1938.

\subsection{0}

The "Cushing ritual" and the Origins of Wilder Penfield's Surgical Technique

Mark C. Preul, William Feindel (Montreal, Quebec) 
Wilder Penfield left two great legacies: the development of successful surgical treatment of epilepsy and the establishment with his colleagues of the Montreal Neurological Institute as a world-renowned medical center, "dedicated to relief of pain and suffering and to the study of neurology."

Penfield's intellectual approach to the nervous system was derived from his studies with Sherrington, Holmes, Cajal, and Hortega. His eclectic surgical style emerged from his familiarity with the operating techniques of Halsted, Dandy, Horsley, Sargent, Cushing, Frazier, Whipple, Leriche, and Foerster. Penfield's debt to these teachers is documented in his memoirs and in an unpublished report on European neurosurgery which he sent to the Rockefeller Foundation in 1928. That Harvey Cushing's surgical ritual (which stemmed from the painstaking operative methods of Halsted) played a paramount role in the origins of Penfield's surgical technique is revealed by a set of notes and drawings by Penfield during repeated visits in the 1920s to Cushing's clinic at the Peter Bent Brigham Hospital.

\section{0-11}

\section{No Man Alone: Wilder Penfield and the Origin of the Canadian Neurological Society}

W Feindel (Montreal, Quebec)

Background: Wilder Penfield, one of the founders of the Canadian Neurological Society (CNS), was helped by many others in planning a preliminary Foundation Meeting on June the 22nd, 1948 in Toronto and the inaugural meeting that took place in Montreal, May 20-21, 1949.

Method: Letters, minutes and portraits concerning the origin of the CNS have been extracted from the Wilder Penfield Archive and the Neuro Archives of McGill University.

Observations: The program of the meeting, planned by Donald McEacherny, established from the outset the interdisciplinary character of the CNS from a symposium on lesions about the foramen magnum to a report on Elliott's brain irrigating solution and a guest lecture by Sydney Sunderland on nerve regeneration. The bilingual aspect of the Society was reflected by program sites at the Montreal Neurological Institute and l'Université de Montréal, with a special lecture by Hans Selye, and by the annual dinner organized by Jean Saucier at Cercle Universitaire; Kenneth McKenzie was toastmaster and Wilder Penfield gave a scholarly presidential talk on "Neurology in Canada and the Osler Centennial".

Conclusion: The inaugural meeting of the Canadian Neurological Society successfully achieved its goal to draw together for the future the many disciplines relating to the practice, teaching and science of neurology.

\section{$0-12$}

Neurosurgeon as Innovator: William V. Cone (1897-1959)

Mark C. Preul, Joseph Stratford, Gilles Bertrand, William Feindel (Montreal, Quebec)
Few neurosurgeons have possessed the prolific ingenuity of William Cone. In 1934, he and Wilder Penfield were cofounders of the Montreal Neurological Institute where, until 1959, he was neurosurgeon-in-chief and neuro-pathologist. Because he did not find writing easy, many of his technical inventions and refinements remained unpublished. His numerous innovations included the extensive use of twist-drill technique for biopsy, drainage for subdural hematoma and cerebral abscess, and ventriculography. In the mid-1940s, he developed power tools driven by nitrogen that led to the modern, universally used air-driven tool systems. He had a special interest in the treatment of spinal dysfunction, for which he invented the Cone-Barton skull-traction tongs and the Cone spinal operating table; and he devised operative procedures for vertebral fracture-dislocation and craniospinal anomalies. For the maintenance of muscle tone in the paralyzed bladder, he constructed a tidal drainage system. $\mathrm{He}$ introduced and popularized ventriculo-peritoneal shunting techniques and carried out some of the earliest experimental trials to treat brain infections with antibiotics. He designed his own set of surgical suction devices, bone rongeurs, and a personal suction "air-conditioning" system for each surgeon. He had a keen interest in intracranial tumors and demonstrated on monkeys how subdural mass lesions caused pupillary dilatation. His work for the military during World War II on effects of altitude on brain pressure remained classified for many years. The first clipping and excision of an intracranial aneurysm is attributed to Cone by Dandy. Although Penfield was known as "the Chief," Cone was referred to as "the Boss." His accomplishments, inventions, and his example as a teacher are part of neurosurgery's legacy.

\section{0-13}

Arthur Roland Elvidge (1898-1985): Contributions to the Diagnosis of Brain Tumors and Cerebrovascular Disease

Mark C. Preul, Joseph Stratford, Gilles Bertrand, T. Dagi, William Feindel (Montreal, Quebec)

The contributions of Arthur Elvidge (1898-1985), Wilder Penfield's first neurosurgical recruit, to the development of neurosurgery have been relatively neglected. His work in brain tumors extended the previous work of Percival Bailey and Harvey Cushing. He published rigorous correlations of clinical and histological information and formulated a revised, modern nosology for neuroepithelial tumors, including a modern histological definition of a glioblastoma multiforme. Well ahead of his time, he held that a glioblastoma was not strictly localized, and was the first to comment that the tumor frequently showed "satellitosis." He was the first neurosurgeon in North America to use angiography for the radiographic diagnosis of cerebrovascular disease. Having studied with Egas Moniz, he was the first to detail the use of angiography for demonstrating cerebrovascular disorders specifically, believing that it would make possible routine surgery of the intracranial blood vessels. Seeking to visualize all phases of the angiogram, he was the impetus behind the design of one of the first semi-automatic film changers. Elvidge, independently along with Moniz, made the first observations on 
thrombosis of the carotid vessels. Elvidge elucidated the significance of embolic stroke and commented on the ischemic sequelae of subarachnoid hemorrhage. Beyond neurosurgery, he co-discovered the mode of transmission of poliomyelitis. Elvidge's soft spoken dry wit and candor, mastery of the understatement, love of exotic travel, and consummate dedication to neurosurgery made him a favorite of patients, neurosurgery residents, nurses and other staff. His accomplishments and example as a teacher and physician have become part of neurosurgery's advancing legacy.

\section{GENERAL NEUROLOGY}

\section{1-01}

\section{Combined Fludrocortisone and Midodrine in Treating Orthostatic Hypotension}

JD Stewart, A Al-Asmi (Montreal, Quebec)

Objective: To determine, in treating orthostatic hypotension $(\mathrm{OH})$, whether combined therapy with fludrocortisone (FC) and midodrine (MD) is more effective than $\mathrm{FC}$ alone.

Background: $\mathrm{FC}$ and MD are often effective in treating $\mathrm{OH}$, but is a combination even more effective?

Design/Methods: Retrospective review of patients treated for moderate-severe $\mathrm{OH}$. $\mathrm{OH}$ control was judged bad, fair, good, or excellent based on symptoms and activities of daily living.

Results: Fifteen patients had symptomatic $\mathrm{OH}$ caused by progressive autonomic failure (7), multiple system atrophy (5), diabetes (3). All used non-pharmacologic treatments for $\mathrm{OH}$. FC was usually chosen as the starting medication. Thirteen patients were treated initially with increasing doses of FC; five of these achieved good or excellent control. Eight remained symptomatic and increasing doses of MD were added; six achieved goodexcellent $\mathrm{OH}$ control. Two patients were treated initially with increasing doses of $\mathrm{MD}$ alone, then $\mathrm{FC}$ alone, then required both to achieve fair control (1) and excellent (1).

Conclusion: FC alone in appropriate doses is adequate therapy for moderate to severe $\mathrm{OH}$ in a minority of patients. The combination of $\mathrm{FC}$ and MD is very effective in patients not responding to $\mathrm{FC}$ alone.

\section{$1-02$}

Isolated Trigeminal Neuropathy Due to Trigeminal Nerve Root Hemorrhage

S Almeida, C Chalk, J Minuk, R Del Carpio, M Guerin, M Levental (Montreal, Quebec)

Background: Isolated trigeminal neuropathy may be traumatic, inflammatory, or idopathic. We report an unusual case caused by a trigeminal nerve root hemorrhage.

Methods: A 62-year-old man felt unwell when walking into his house, abruptly lost consciousness, and fell to the floor. Several minutes later, he was responsive, and was taken to hospital. He had a left scalp hematoma and EKG changes of acute myocardial ischemia. Intravenous streptokinase was given. Left facial numbness developed soon after admission, with loss of col and pinprick sensation throughout the left trigeminal distribution, especially over the cheek, and an absent left corneal reflex. Otherwise the neurological examination was normal. Nine months later, he was unchanged.

Results: Cranial CT showed hemorrhage in the cisternal portion of the left trigeminal nerve. MR imaging 2 and 8 months later showed hemosiderin along the nerve sheath but no other abnormalities.

Conclusion: Isolated trigeminal neuropathy due to hemorrhage into trigeminal nerve tumours or from lateral pontine vascular malformations has been described, but our patient had no underlying structural lesion. We assume the lesion was caused either by direct trauma to the trigeminal nerve root at the time of the fall, or was a localized hemorrhagic complication of streptokinase.

\section{$1-03$}

\section{Leptomeningeal Adenocarcinomatosis Presenting As Bilateral Central Scotomata}

J. Politsky, A. Silas, J. Flint, J. DeNegri, P. Bratty (Vancouver, British Columbia)

The clinical presentation of a 36 year old male with leptomeningeal and lymphangitic carcinomatosis secondary to moderate to well-differentiated primary lung adenocarcinoma is reported.

This patient presented to hospital with a two month history of progressive, bilateral central visual loss, which was mistaken for optic neuritis. One month prior to admission, cerebrospinal fluid (CSF) analysis failed to reveal malignant cells. Concurrent chest $x$-ray, head CT and MRI exams were normal. Following these investigations, the patient developed facial weakness, hearing loss, and imbalance. Repeated cytologic CSF analysis after admission was also negative.

The diagnostic quartet of HA typical of raised intracranial pressure, blindness, absent or sluggish pupillary responses, and normal optic discs, indicative of leptomeningeal optic nerve involvement, did not aid in this patient's diagnosis. Leptomeningeal carcinomatosis presenting with bilateral central scotomata is exceedingly uncommon.

This case had a number of other atypical features, as well, including the patient's young age, the early meningeal seeding, and the presence of malignant cells only on the third lumbar puncture.

This case serves to expand the literature with regard to the clinical presentation of carcinomatous leptomeningeal invasion. A review of the clinical, laboratory, and radiologic diagnosis and pathophysiology of this entity is discussed.

\section{$1-04$}

Nocardia asteroides Cerebral Abscesses in NonImmunocompetent Hosts: A Report of Three Cases and Review of Medical and Surgical Management

Ian G. Fleetwood, John M. Embil, Ian B. Ross (Winnipeg, Manitoba)

Background: Nocardia asteroides, an aerobic actinomycete 
common in soil, is a rare cause of primary or metastatic cerebral abscess. The overall incidence of cerebral nocardiosis is increasing with increasing rates of immunosuppression.

Methods: We report three cases of Nocardia asteroides brain abscesses in non-immunocompromised hosts, two of whom presented with single intracranial lesions and no evidence of extraneural disease. Both were admitted to hospital with presumptive diagnoses of high grade gliomas. The third patient had multiple cerebral lesions and pulmonary involvement.

Results: The management of all three cases is discussed, specifically medical and surgical aspects.

Conclusions: Nocardial brain abscesses are often diagnosed late due to a low index of suspicion and clinical presentations common to other diagnoses. Blood cultures are often negative. They have mortality rates greater than three times that of abscesses due to other causes. Although trimethoprim-sulfamethoxazole is considered the antimicrobial of choice, rifampicin, imipenem, minocycline, cefotaxime and amikacin have been used effectively, either alone or in combination with each other. There are reports of Nocardia asteroides being resistant to all these agents. We advocate open or stereotactic biopsy early in the management of these patients in order to facilitate prompt specific antimicrobial coverage.

\subsection{5}

\section{Central Nervous System Complications of Septic Cavernous} Sinus Thrombosis

\section{T.W.J. Watson, M. Omojola (Riyadh, Saudi Arabia)}

Background: Septic cavernous sinus thrombosis (SCST) is a recognized complication of infections of mid-facial and periorbital areas and sinusitis. SCST is associated with CNS complications that require early recognition and aggressive management.

Method: Case presentation illustrating a combination of complications of SCST with literature review of diagnosis, investigation and management of SCST and its complications.

Results: A 38-year-old female presented with fever, proptosis, complete VI N palsy, and partial III $\mathrm{N}$ palsy accompanied by sudden onset of $R$ hemiparesis and aphasia following 14 days of topical antibiotics for a purulent $\mathrm{L}$ conjunctivitis. CT and $M R I$ revealed multiple enhancing lesions in the $L$ frontal and temporal lobes with enhancing subdural fluid collection. Angiography showed aneurysm of the cavernous portion of the $\mathrm{L}$ internal carotid (IC) and irregular tapering occlusion of the $\mathrm{L}$ middle cerebral artery. Drainage of empyema and L temporal abscess yielded culture growth of Strep viridans milleri. Repeat angiogram revealed enlargement of the aneurysm managed with balloon occlusion of the proximal IC. Treatment with IV ceftriaxone, metronidazol and decadron resulted in nearly complete resolution of aphasia with mild residual weakness in the $R$ upper extremity at 6 month follow up.

Conclusions: In the modern era of antibiotic use SCST has become less common but remains a potentially fatal complication of sinusitis and infections of the mid-facial and periorbital areas. The pathophysiology relates to the valveless system of extra to intracranial venous drainage, and the potential for direct and/or arterial extension of infection. This case report and review of the literature emphasize the major potential complications of SCST and suggest guidelines for early diagnosis and management.

\section{1-06}

\section{Cervical Myelopathy Associated With Fetal Alcohol} Syndrome

G. Blevins, E. Wirrell, K. Tong, C. Voll (Saskatoon, Saskatchewan)

Introduction: Cervical vertebral anomalies resembling Klippel-Feil syndrome have been reported in fetal alcohol syndrome (FAS). To our knowledge, association with spinal cord compression has not been described. We report a 39 year old male with FAS and a progressive cervical myelopathy.

Case Report: A 39 year old mildly mentally handicapped male, who had been diagnosed with FAS in childhood, presented with a twelve month history of progressive difficulty walking and stiffness of all four extremities. On examination he had clear features of FAS including microcephaly, short stature, short palpebral fissures, long philtrum and a thin vermillion border. His neck was short with limited lateral rotation. Neurological examination showed spasticity in all extremities with hyperreflexia and bilaterally upgoing toes. MRI of the cervical spine showed a Klippel-Feil variant with basilar invagination, severe craniocervical junction canal narrowing and a syrinx which extended from $\mathrm{C}_{1}$ to $\mathrm{C}_{2-3}$.

Conclusion: Patients with FAS and cervical vertebral anomalies are at risk of developing cervical myelopathy and should be monitored for this complication.

\section{1-07}

\section{A 26-Year-Old Woman with Focal Varicella Zoster Encephalitis}

G. Blevins, A. Kirk, K.Tong, D. George (Saskatoon, Saskatchewan)

Background: Focal encephalitis due to varicella zoster virus (VZV) has been described involving structures such as the optic tract or trigeminal nucleus. VZV can result in vasculitis and infarction, especially in the pediatric or immunosuppressed population. Unlike herpes simplex virus (HSV), focal encephalitis involving the temporal lobe has not been described, to our knowledge. We present the case of a patient with focal temporal lobe VZV encephalitis.

Methods: A 26-year-old woman presented with a three-week history of 'stiff neck' and three spells of olfactory auras. The third episode of olfactory aura was followed by a secondarily generalized seizure. There was a history of childhood chickenpox but no recent vesicular skin lesions. Examination did not reveal focal neurological signs. Initial CT scan demonstrated a low-density lesion in the left frontotemporal lobe with focal meningeal enhancement. MRI confirmed the lesion, notably 
sparing the putamen. The patient subsequently underwent left temporal tip lobectomy. Polymerase chain reaction for HSV on tissue was negative. Pathological examination revealed lymphocytic meningoencephalitis with slight focal necrosis, reactive astrogliosis and perivascular lymphocytic inflammation. Immunostaining for HSV I, HSV II and cytomegalovirus were all negative. VZV was isolated from tissue submitted for viral culture.

Results: The patient was treated with intravenous acyclovir. Following completion of two weeks of therapy, the patient was found to remain asymptomatic. Follow-up CT scan showed radiological improvement.

Conclusions: We demonstrate a case of focal VZV encephalitis involving the temporal lobe in an immunocompetent patient. Although HSV is well known to produce such an encephalitis, VZV has not been previously described with this presentation. We suggest that VZV infection should be considered in the setting of suspected focal encephalitis where investigations are negative for HSV infection.

\section{$1-08$}

\section{Stomatologic Complication of Giant Cell (Temporal) Arteritis}

D. Pelletier, M. Thibault, R. Morency, J-P. Bouchard (Québec City, Québec)

Background: Giant cell arteritis (GCA) is a vasculitic disorder well known for its diversity of neurologic and non-neurologic clinical presentations. Tongue necrosis has been described as a GCA complication and is well documented. We report a patient who had buccal ulcers as an early manifestation of GCA symptoms without tongue involvement.

Case history: A 73-year-old woman with medical history of heart disease, osteoporosis and acute glaucoma resulting in left ocular blindness years ago presented painful and bilateral ulceration in her mouth two weeks before neurologic consultation. She had never suffered from mouth ulceration in her life. Gradually over weeks, she clearly experienced a new left frontal headache, jaw claudication, scalp tenderness, anorexia, and fatigue without polymyalgic symptoms. Sedimentation rate (ESR) was $79 \mathrm{~mm} / \mathrm{hr}$. Examination revealed pulseless and thickened left temporal artery. Three large mouth ulcers were present, one on either side of her maxillar tuberosities and a third one at the angle of the left mandible. The patient was toothless. Neurological exam was within normal except for cecity of her left eye. She was given oral corticosteroids and complete healing of ulcerations appeared only when ESR was normalized along with systemic symptoms.

Investigation revealed inflammatory anemia and positive bilateral giant cells arteritis at temporal biopsy. A biopsy of two oral ulcers at the tuberosities revealed an extensive acute and chronic necrotizing vasculitis without fibrinoid necrosis.

Conclusion: Not recognizing some of the rare neurological and non-neurologocal clinical manifestations of GCA can delay the diagnosis and treatment of that disorder and cause additional morbidity.

\subsection{9}

\section{Case Report: Fulminant CNS Vasculitis in SLE}

A Hanson, D Howse, S Ludwin, R Smith, P Ford (Kingston, Ontario)

Frank CNS vasculitis is rare in SLE. Neurological deficits are usually attributed to a vasculopathy or antiphospholipid mediated thrombosis. We report an unusual case of autopsy proven CNS vasculitis in a patient with SLE.

This 46-year-old female with SLE developed an aggressive neurological illness characterized by multiple hemorrhagic infarcts in the CNS. She presented initially with acute paraplegia. Seizures and a deteriorating level of consciousness ensued over the next 7 days. This was ultimately fatal.

MRI of her spinal cord showed hematoma extending from her cord at T10. Serial CT head scans showed bilateral PCA infarcts, hemorrhage in the corpus callosum and subarachnoid blood. Cerebral angiography showed extensive segmental areas of changes in luminal diameter classic for vasculitis. Despite high dose corticosteroids and cyclophosphamide, she went on to develop bilateral ACA, subcortical and cerebellar infarcts.

Autopsy showed extensive infarction with resolving hematoma in the spinal cord, bilateral cerebral infarcts and resolving hematoma in the corpus callosum. Microscopic examination of the main cerebral arteries showed extensive focal scarring of the media, adventitia and elastica suggestive of healing vasculitis. Similar vessels were seen in the spinal subdural space. This is a rare and unusual presentation of fulminant CNS vasculitis in SLE.

\section{1-10}

\section{A Database for Clinical Research in Multiple Sclerosis}

\section{Duquette P, Lebrun-Crête H (Montreal, Quebec)}

Canada is a country with a high prevalence of multiple sclerosis (MS). Canadian scientists are at the forefront of research in MS. To further enhance its research effort, the Canadian Network of MS Clinics will seek funding for the establishment of a common database that will pool data from close to 30,000 patients followed in individual clinics across the nation. We will take advantage of the experience acquired by similar groups (stroke, Alzheimer, epilepsy). The information gathered with the database will be used, on one hand, to describe the condition and the needs of the MS population. On the other hand, it will be used in both global and more focused epidemiological studies. Given the magnitude of the data, we should be in a good position to bring definitive answers to pressing questions. In this way, the Network hopes to continue its contribution to the quest for the cause and the cure of MS, as well as the improvement of the care of the patients. The structure and goals of the database will be described. The main published studies of the Network will be summarized. 


\section{$1-13$}

A Pilot Study of the Effect of Weekly Intramuscular (IM) 6.0 MIU Interferon beta-1a (IFNB-1a) on Monthly Gadolinium-enhanced (Gd+) Brain MRI Activity in Patients with Relapsing Multiple Sclerosis (MS)

Waubant E, Sloan R, Vermathen M, Rooney W, Andersson PB, Stewart T, Chan A, Hietpas J, R., Weiner M, Goodkin D (San Francisco, California)

Objective: Compare the number and volume of new $\mathrm{T} 1 \mathrm{~W}$, $\mathrm{T} 2 \mathrm{~W}$ and $\mathrm{Gd}+$ lesions on monthly MRI before and after initiating weekly IM 6.0 MIU IFNB-la.

Background: IFNB-lb subcutaneous every other day and IFNB-I a subcutaneous 3 times/week reduce monthly $\mathrm{Gd}+$ activity in patients with relapsing MS. The effect of weekly IFNB-la 6.0 MIU IM on monthly Gd+, TIW and T2W MRI activity has not been rigorously evaluated.

Design/Methods: 7 female and 2 male patients who participated in a natural history study of MRI activity and had 6 monthly scans with 0.5 new $\mathrm{Gd}+$ lesions/month started weekly IFNB-Ia 6.0 MIU IM. Monthly scans were continued for 6 additional months and mean MRI activity for each patient was compared during pre and on-treatment phases. 8 patients had relapsing remitting MS and 1 had secondary progressive MS with relapses.

Results: The mean number of new pre-treatment lesions/patient was: 1) 6.0 , 2) $1.3,3$ ) 3.2 , 4) $2.7,5) 4.7,6$ ) 0.6 , 7) 0.7, 8) 1.0, 9) 1.2. Data on-treatment and rate of $\mathrm{T} 1 \mathrm{~W}$ lesions rising from new $\mathrm{Gd}+$ or $\mathrm{T} 2 \mathrm{~W}$ lesions pre and on-treatment will be presented.

Conclusion: This study will provide information regarding the effect of IFNB-1a IM 6.0 MIU on monthly new Gd+, T1W and T2W MRI lesions in patients with relapsing MS.

\section{1-14}

\section{Charting by Exception: A Pilot Project on Neurosciences}

L Kelloway, V Edmonds (Mississauga, Ontario)

\section{CHILD NEUROLOGY}

\section{2-01}

Congenital Focal Alopecia as an Early Marker for Syndromes of Brain Dysgenesis

\section{E.J. Donner, D.L. MacGregor (Toronto, Ontario)}

Background: Neurocutaneous disorders present with variable combinations of skin, hair, ocular, vascular and central nervous system abnormalities. Congenital focal alopecia may be present in these syndromes and represent an easily identified marker of brain dysgenesis.

Methods: We have reviewed three cases presenting to our pediatric neurology clinic with neurocutaneous syndromes accompanied by congenital focal alopecia.

Results: Patient 1 presented with focal seizures, mild hemipare- sis, and developmental delay. Magnetic resonance imaging (MRI) revealed an arachnoid cyst and pachygyria underlying an area of congenital focal alopecia. Patient 2 presented with mild hemiparesis, global developmental delay, hyperpigmentation and congenital patchy alopecia. MRI showed ventriculomegaly. Hemiparesis contralateral to the alopecia was suggestive of a microscopic migration defect. Patient 3 presented with focal seizures, a linear sebaceous nevus and congenital patchy alopecia. MRI revealed hemimegalencephaly ipsilateral to the cutaneous findings.

Conclusions: The neurocutaneous syndromes may represent a spectrum of disordered embryogenesis. Postulated mechanisms include early mesodermal defects which result in secondary ectodermal defects. Congenital focal alopecia may be a marker for brain dysgenesis associated with such disorders as encephalocraniocutaneous lipomatosis, linear sebaceous nevus syndrome and incontinentia pigmenti syndrome.

\section{2-02}

Clinical Manifestations of Cerebellar Vermal HypoplasiaJoubert Variant

S. Lindsay, D. Chan, S. Chuang, D. L. MacGregor (Toronto, Ontario)

Background: Joubert syndrome (JS) is an autosomal recessive disorder characterized by a partial or complete absence of the cerebellar vermis and ataxia, episodic tachypnea/apnea in infancy, developmental delay and abnormal eye movements. Some patients have retinal dystrophy, dysmorphic features, renal and liver disease. The objectives of the review were to evaluate the association of vermal hypoplasia with JS and other developmental disorders without the full clinical picture of JS as well as re-evaluate the classification of cerebellar dysgenesis.

Methods: Ten patients diagnosed with clinical JS or with radiological evidence of vermal hypoplasia (excluding DandyWalker malformation) were reviewed.

Results: We found that patients with neuroimaging studies with pathology similar to that found in JS had different clinical phenotypes. One child had normal neurological function. The principal features in our series were developmental delay $(n=8)$, hypotonia $(n=5)$, abnormal ocular movements $(n=6)$, and respiratory abnormalities $(n=3)$. One child had hepatic fibrosis and four had renal disease (polycystic and tubulointerstitial disease). Two patients had been labelled JS clinically but did not fit the radiologic criteria as the inferior vermis was present though hypoplastic. These findings suggest there may be JS variants.

Conclusions: 1) There is significant variability in the manifestations of cerebellar vermal hypoplasia and associated neurodevelopmental disorders. 2) The classification of cerebellar malformations should include JS variants.

\section{2-03}

Nocturnal Paroxysmal Dystonia (NPD) in Children: A Partial Epilepsy Syndrome with Frontal Lobe Seizures or Sleep Disorder

D.L. MacGregor, W. J. Logan (Toronto, Ontario) 
Background: NPD is described as complex motor attacks occurring abruptly during sleep. There can be dystonic posturing, tonic limb and trunk movement, automatisms and vocalizations. Short duration and more prolonged events are described. EEG, has been reported in some patients to demonstrate epileptic activity from the mesial frontal regions. There is good response to Carbamazepine or Clonazepam.

Methods/Results: A 13 year old boy presented for review of his long standing epilepsy having had no seizures reported for 2 years maintained with good therapeutic levels on Carbamazepine and Primidone. Onset had been at 11 weeks of age with intractable seizures from 6 months of age and a significant episode of status epilepticus at 9 months. Neurological examination was normal except for cognitive delay. At 10 years of age, his parents began describing "night startles" - having fallen asleep, he would sit upright, appear panicked (later describing that he felt "scared"). EEG showed excessive slowing with bifrontal independent epileptiform discharges, more active on the right.

Overnight sleep study showed 2 arousals from slow wave sleep during which he displayed dystonic posturing, choreoathetoid movement of the right arm and head rotation lasting 1 minute with ending in confused wakefulness. EEG with expanded frontal temporal montage did not detect sleep related seizure activity. Addition of Clonazepam to his treatment regime resulted in a marked reduction in the frequency and intensity of these nocturnal events.

Conclusion: The occurrence of the nocturnal events described in this child indicated intractable epilepsy even with a non-epileptic EEG. NPD has been considered either a sleep disorder or nocturnal epileptic seizures of frontal lobe origin. Recognition of these events as epileptic will allow appropriate diagnosis and therapy.

\section{2-04}

\section{Mycoplasma Pneumonia Encephalopathy in the Absence of Abnormal CSF Findings}

\section{Keegan, N. Lowry, J.Y. Yager (Saskatoon, Saskatchewan)}

Mycoplasma pneumoniae encephalitis is a recognized cause of reversible coma in children. The fact that this entity can occur in the presence of a normal cerebrospinal fluid examination is less well appreciated. We report 2 children who presented with coma who had normal lab investigations including CSF with evidence of acute Mycoplasma pneumoniae infection.

The first case is a 10 year old girl who presented with a 2 week history of non-productive cough, low grade fever and headache. This was followed by a 2 day history of poor attentiveness. She then had a secondarily generalized seizure, became comatose, and was admitted to hospital. Two CSF examinations were normal. Electroencephalograms (EEG) showed generalized polymorphic slow waves at 1 hertz (hz) frequency. She remained comatose for 3 days but subsequently recovered rapidly. Serology was strongly positive for Mycoplasma pneumoniae.

The second case is a 10 year old boy who had a 2 week prodrome of lethargy, anorexia, vomiting and mild cough. He then had a generalized tonic-clonic seizure and became comatose. CSF was normal. EEG showed generalized slowing at $1 \mathrm{hz}$.
Cold agglutinin test was positive as were IgM Mycoplasma pneumoniae antibodies. He improved rapidly over 3 days.

These illustrated cases exemplify Mycoplasma pneumoniae as a cause of coma in the setting of a normal CSF. The finding suggests the ability of this organism to induce an encephalopathic process via a toxin, rather than through direct invasion of the CNS. Furthermore, the finding of a normal CSF may herald a more favorable prognosis.

\section{2-05}

\section{Thoracic Outlet Syndrome in Childhood}

\section{E.H.Roland, R.D.Keyes (Vancouver, British Columbia)}

Background: Thoracic outlet syndrome refers to a spectrum of neuromuscular abnormalities involving the upper limbs which are related to compression of the lower brachial plexus usually by a cervical rib, elongated transverse process of lower cervical vertebrae or fibrous bands. Although this syndrome has been reported in adults, the clinical and electrophysiological features and outcome in children have not been well described.

Methods: We describe the clinical features, diagnostic procedures, treatment and outcome in 4 children with thoracic outlet syndrome.

Results: The patients included 3 girls and 1 boy. Symptoms included progressive numbness and paresthesiae of hands (3), unilateral pain in shoulder and hand (2), ipsilateral small hand with muscular atrophy (2). Symptoms were right-sided in (2 patients), left-sided (1), bilateral (1). Onset of symptoms was insidious, recognized after 9 years of age (range: 9-14 yrs). On examination, hyperabduction and Adson's maneuvres resulted in decreased pulse in 2 patients. Electromyography/nerve conduction studies demonstrated post-ganglionic abnormality involving $\mathrm{C}_{8}-\mathrm{T}_{1}$ fibres with no evidence of carpal tunnel syndrome or ulnar neuropathy. All patients had normal plain $\mathrm{X}$-rays as well as MRI of the cervical spine and thoracic outlet. Doppler studies of vessels in the upper extremities with hyperabduction maneuvres demonstrated asymmetric waveform amplitudes without significant arterial compression. Two patients had improvement of symptoms and neurophysiological studies following physiotherapy. One patient had transaxillary surgical resection of the first rib with resolution of symptoms. One patient declined therapy and symptoms persisted.

Conclusions: Thoracic outlet syndrome is a rare cause of progressive neuromuscular problems involving the upper limbs in childhood. Accurate diagnosis and management are especially problematic in this age group.

\section{2-06}

Bilateral Middle Cerebral Artery Infarction in the Newborn

EH Roland, K Poskitt, BA Lupton, A Hill (Vancouver, British Columbia)

Background: Perinatal focal arterial cerebral infarction in the newborn is most commonly unilateral and involves the territory of a middle cerebral artery. We describe the clinical features and 
short-term outcome in 4 newborns who had bilateral middle cerebral artery infarction.

Methods: Four newborns had bilateral middle cerebral artery infarction diagnosed by fetal ultrasound or computed tomography. All had neurological follow-up for 2-4 years.

Results: Affected infants (2 female, 2 male) had gestational ages ranging from $24-42$ weeks. In 3 cases, bilateral middle cerebral artery infarction occurred during the intrauterine period, 1 case had normal serial postnatal cranial ultrasound scans until 10 weeks of age. Prenatal complications included prematurity (3), twin pregnancy (2) with death of a co-twin (1), maternal drug use (1), alloimmune thrombocytopenia (1). Two infants had other congenital anomalies: syndactyly, absent pellucidum, optic nerve hypoplasia. Postnatal complications included E.coli meningitis and candida sepsis (1), progressive hydrocephalus (1). Studies for hypercoaguable conditions were negative. All newborns were lethargic and had feeding problems; none had seizures. Neurological sequelae included major cerebral palsy (2), microcephaly (2), epilepsy (2), mild developmental delay (2).

Conclusions: Bilateral middle cerebral artery infarction is an uncommon occurrence in the newborn which may relate to numerous prenatal and postnatal etiologies. The stage of maturation of the immature brain may be a major determinant for the bilateral distribution of injury. Short-term neurological outcome is variable.

\subsection{7}

Unusual Metabolic and Renal Abnormalities Associated with Merosin Negative Congenital Muscular Dystrophy

E.H. Roland, D.K. Hewes, L. Clarke, R.J. Adderly, A.F. Cogswell (British Columbia)

Background: Congenital Muscular Dystrophy (CMD) is diagnosed clinically on the basis of weakness from birth and histological abnormalities consistant with muscular dystrophy. Several phenotypes may be identified based on central nervous system involvement and the presence or absence of laminins (merosin). Laminins are extracellular matrix proteins in basement membranes of skeletal muscle, Schwann cells of peripheral nerve and kidney.

Methods: We describe unusual metabolic and renal derangements in a child with merosin negative CMD.

Results: This boy is wheelchair bound and has normal cognitive function. At 15 months, he had one seizure, persistent ketosis and required ventilation for several days because of pneumonia. Computed tomography demonstrated extensive decreased attenuation in cerebral white matter. At 5 years, he developed pneumonia with acute respiratory failure which required ventilation. He had encephalopathy, transient hypoglycemia and persistant ketosis, as well as transient hyponatremia, despite appropriate fluid management, consistant with renal tubular wasting of sodium. Total and free carnitine levels were very low (14.0 and $3.9 \mathrm{umol} / \mathrm{L}$; Reference ranges: $30-69$ and $16-60 \mathrm{umol} / \mathrm{L})$. He receives ongoing, continuous $\mathrm{G}$-tube feeds, carnitine supplementation and noninvasive BiPAP ventilation.
Conclusions: This boy developed unusual metabolic derangements associated with merosin negative CMD which included ketotic hypoglycemia, carnitine deficiency and renal tubular dysfunction. The ketotic hypoglycemia presumably reflects impaired tolerance of fasting in a catabolic state due to low glycogen storage in reduced muscle mass. Furthermore, merosin deficiency in kidneys may predispose children with CMD to renal tubular dysfunction.

\section{2-08}

A Clinical Improvement Project in Neurology Clinic: Measuring Outcomes of a Change in Care Delivery to Children with Epilepsy

J.R. Boyd, D.L. MacGregor, L.J. MacMillan, E.J. McCabe, W. Schoonheyt, B.L. Zimnowodzki (Toronto, Ontario)

Background: Attendance at a clinical improvement workshop provided a neurology multidisciplinary team with practical methods for carrying out a small change project. A clinical improvement test change was carried out to reduce routine therapeutic drug monitoring in children with epilepsy attending an ambulatory clinic at the Hospital for Sick Children.

Methods: Outcome measures relating to function, cost, clinical outcomes, and satisfaction were identified using a "Clinical Value Compass". The current process of care delivery was analyzed, and redesigned to eliminate routine AED testing and incorporate individualized assessment of the need for AED levels. A planned test change was implemented over one month, and outcome measures were evaluated for evidence of improvement.

Results: The change in practice resulted in a reduction of bloodwork by $50 \%$. Consequently, lab costs, phlebotomy time, children's anxiety and discomfort associated with bloodwork, family waiting time, and follow-up time were reduced; and client satisfaction, and involvement in decision-making were increased. All drug levels drawn were therapeutic, and no adverse clinical outcomes on children who did not have AED levels performed were reported.

Conclusions: The quality and value of health care for children with epilepsy was measurably improved by a clinical improvement change project. Multidisciplinary collaboration, and the use of a model for linking outcome measures with process improvement facilitated timely, measurable improvements to care delivery in the Neurology Clinic.

\section{2-09}

Male Rett Syndrome Variant: Application of The Diagnostic Criteria

MMS Jan, JM Dooley, K Gordon (Halifax, Nova Scotia)

Background: Classic Rett syndrome (RS) has been described in females only. Although an $\mathrm{X}$ chromosome origin is probable, it has not been substantiated. It is possible therefore that RS could occur in males. 
Method: Case report of a male with RS and review of all the reported cases in the literature. We compare our case to the other cases, and examine the applicability of the classic RS diagnostic criteria to this variant.

Results: To date, nine cases of male RS have been reported. We describe a further male who met 7 of 9 necessary criteria and 6 of 8 supportive criteria as defined by the RS Diagnostic Criteria Work Group. When we apply these criteria to the other 9 reported cases, many necessary inclusion criteria were not met despite the absence of exclusion criteria. The supportive criteria were even more variable and limited in many cases.

Conclusions: Males with RS appear to represent a heterogeneous phenotype, with clinical features that may meet many but not all of the necessary diagnostic criteria of classic RS. Less restrictive criteria are needed to include this variant which should be considered when evaluating males with idiopathic developmental regression, autistic features, and loss of hand function.

\section{2-10}

Facioscapulohumeral Dystrophy: Autosomal Recessive Facio-scapulohumeral Dystrophy with Longstanding Unilateral Scapular Winging: a New Syndrome?

P.Jacob, P. Humphreys, M. Speevak, M. McGill (Ottawa, Ontario)

Facioscapulohumeral muscular dystrophy predominantly affects the shoulder girdle and the facial muscles. In some cases, the pattern of weakness involves the lower extremities as well. The pattern of inheritance is autosomal dominant but sporadic cases have been encountered. In $80 \%$ of patients with AD-FSH the gene responsible for the condition has been localised, by linkage studies, to chromosome $4 \mathrm{q} 35$.

We report two cases of apparently sporadic FSH, presenting with longstanding unilateral scapular winging (years) and significant asymmetrical evolution early on. Both occurred in females with onset of scapular winging early in the second decade. Detailed physical examinations, family histories, biochemical and neurophysiological studies as well as muscle biopsy (in one patient) confirmed the diagnosis of muscular dystrophy with clinical features of the facioscapulohumeral type. DNA analysis of patients and parents are in progress. Formal evaluation of both sets of parents showed them to be unaffected, suggesting an autosomal recessive pattern of inheritance or a spontaneous mutation.

The long duration of apparently non-progressive unilateral scapular winging in the present cases as well as the lack of family history suggests that they may represent a unique presentation of muscular dystrophy distinct from AD-FSH.

The diagnosis of FSH-type muscular dystrophy should be entertained in any patient with unexplained scapular winging even if unilateral in nature.

\section{2-11}

\section{Pachygyria Associated With Childhood-Onset Epileptic Spasms}

E. Wirrell, K. Tong (Saskatoon, Saskatchewan)

Introduction: Epileptic spasms are a rare seizure type which occur in clusters. They resemble infantile spasms but are seen in older children. Previous cases have either been reported in association with Lennox-Gastaut syndrome or have shown definite focal clinical and EEG features. Response to anti-epileptic medication has been poor.

We report a case of symmetrical epileptic spasms occurring in a child who did not have Lennox Gastaut syndrome. A significant reduction in seizure frequency and severity was noted with clobazam monotherapy.

Case Report: A 13 year old boy, with moderate global developmental delay, developed symmetrical epileptic spasms at age 5 years, which increased both in frequency and severity over time. He had a history of three generalized tonic clonic seizures but had never had atonic or nocturnal tonic seizures. Neurological exam was normal except for a prominent startle response to auditory stimuli.

Metabolic workup was unremarkable but MRI showed bilaterally symmetric pachygyria affecting predominantly frontal and parietal lobes.

After failing therapy with valproic acid, he was successfully treated with clobazam, with a marked reduction in seizure frequency.

Conclusion: Pachygyria can cause symmetrical epileptic spasms, and a favorable response to clobazam may be seen.

\section{2-12}

\section{Lorazepam for home treatment of seizures in children}

\section{J. Buckley, S. J. Penney (St. John's, Newfoundland)}

Objectives: To determine if families found this a useful treatment for their child's seizures.

Methods: Lorazepam is available to be administered sublingually, rectally or parenterally for the short-term treatment of seizures. It is a routine procedure in our department to teach parents the use of this medication to control seizures in the home.

Results: Our protocol will be outlined. A review of over thirty-five families who have been taught this method is detailed.

Conclusions: We have found lorazepam a satisfactory treatment for the management of seizures in the home. It has been effective in terminating seizures therefore making it a cost-effective therapeutic option in saving health care resources.

\section{2-13}

\section{Familial Bilateral Occipital Calcification}

DR McLean, A Al-Semari, ME Fiol (Riyadh, Saudi Arabia)

Background: The syndrome of bilateral occipital calcification and epilepsy (BOCE) has been recognized for the past 10 years. 
The etiology remains obscure but half the patients have coeliac disease and folic acid deficiency has been implicated. We report the second familial occurrence of this syndrome.

Case Report: The patient is a 19-year-old Saudi man who developed seizures at age 8 . He has refractory focal and secondarily generalized seizures with progressive intellectual deterioration. His EEG shows generalized spike-wave and multi-focal sharp wave activity. CT and MRI imaging demonstrated bioccipital cortical and subcortical floccular calcification. Small bowel biopsy was normal. His 34-year-old sister has recurring monthly seizures despite medication but is intellectually normal. Her brain CT showed similar but less extensive bi-occipital calcification. There are 6 full siblings and 15 half siblings (different mothers) who are intellectually normal without seizures.

Conclusions: These two siblings demonstrate the spectrum of clinical manifestations in this syndrome. The finding of familial BOCE raises the possibility of a genetic etiology for this syndrome.

\section{$2-14$}

\section{Can Canadian Pediatric Neurologists Develop a Uniform} Countrywide Anticonvulsant Handout

\section{J. Buckley, S. J. Penney (St. John's, Newfoundland)}

Objectives: Canadian Pediatric Neurologists were surveyed to compare the written information that was given to parents when an anticonvulsant medication was prescribed.

Methods: As an example written information on valproic acid a commonly prescribed medication for seizure control will be compared and contrasted.

Results: Differences between centers were identified and will be outlined.

Conclusions: A consensus among Canadian Pediatric Neurologists on what information regarding anticonvulsant medication should be given to parents could lead the way in setting national guidelines which are physician rather than industry driven.

\section{$2-15$}

\section{Neurocytoma: a Not So Benign Tumor}

D. J. Buckley, L. F. Jardine, J. P. Hand, C. Pushpanathan, F. Maroun (St. John's, Newfoundland)

Objective: To report the case of a boy who developed a neurocytoma that was resistant to treatment.

Background: Neurocytomas are recently defined tumors pathologically that classically develop in the lateral ventricles of young people. They are generally benign.

Results: The case of a six year old boy will be presented describing his clinical course leading to his death over a year from diagnosis.

Conclusions: Although this is a very rare brain tumor it is becoming clear that it may not have such a benign outcome as previously thought.

\section{NEUROPHYSIOLOGY}

\section{3-01}

A Unique Case Of Systemic Sclerosis, Inclusion Body Myositis, And Acute Transverse Myelopathy

J. Politsky, A. Silas, I. Mackenzie, C. Patterson, R. Mosewich, V. Sweeney, B. Thiessen (Vancouver, British Columbia)

Background: Systemic sclerosis (SS) is a multi-systemic connective tissue disorder (CTD) characterized by fibroblast activation, excessive collagen production, and obliterative microvascular lesions. Neurologic involvement, usually restricted to the peripheral nervous system, is uncommon. Very rarely, acute transverse myelopathy (ATM) has been described in patients with a CTD. In these cases, diagnostic imaging studies were normal.

An overlap syndrome refers to the coexistence of more than one CTD. The coexistence of SS and inclusion body myositis (IBM) is not a recognized overlap syndrome. The combination of SS, IBM, and ATM, has never been described.

Results: A 39 year old left-handed male, diagnosed 9 years earlier with SS and polymyositis presented to our hospital with a two month history of ascending numbness to the axilla. Investigations confirmed the diagnosis of the CREST variant of SS, IBM with the characteristic pathologic features, and ATM associated with a high signal lesion in the mid-cervical cord on proton density and $\mathrm{T} 2$-weighted magnetic resonance imaging.

Conclusions: The coexistence of SS and IBM may represent a unique overlap syndrome. The presence of ATM in a patient with SS and IBM, which has never been described, may be a helpful clue in the underlying pathogenesis of these conditions.

\section{3-02}

\section{A Case of Guillain-Barre Syndrome and Hodgkin's Disease}

Sian D. Spacey, Lisa Bateman, David Kincaid, Jeff Beckman (Vancouver, British Columbia)

Objective: To present a case of Guillain-Barre Syndrome (GBS) in the setting of Hodgkin's Disease.

Background: GBS is an acute symmetric predominantly motor polyneuropathy typically of cryptogenic origin. GBS is accepted to be an immunologically mediated disease caused by delayed hypersensitivity to the peripheral nervous system myelin or a component thereof. GBS has been associated with campylobacter jejuni infection, viral syndromes, cancers and a variety of other medical conditions. There are a few reports in the literature of an association between GBS and Hodgkin's Disease. We present here a case of GBS in a woman in whom Hodgkin's lymphoma was diagnosed 4 weeks after the onset of the polyneuropathy.

Design: Case report and review of the literature.

Results: A 39 year old woman presented with an ascending symmetrical motor polyneuropathy over a 1 week duration. Clinically the patient had grade 3 motor weakness in her lower limbs and grade $4+$ weakness in her upper limbs and only minimal 
vibratory sensory loss. CSF analysis demonstrated an elevated protein of $1139 \mathrm{mg} / \mathrm{L}$. Eleven courses of plasmapheresis produced little clinical improvement and she was further treated with IVIG 2 $\mathrm{gm} / \mathrm{kg}$ over 4 days. Her condition continued to deteriorate, she was intubated and investigated for a possible alternative diagnosis. CT scans demonstrated a $2.5 \mathrm{~cm}$ splenic mass and paratracheal lymph nodes which, when biopsied,were diagnostic of Hodgkin's lymphoma. EMG and nerve conduction studies demonstrated a predominantly demyelinating polyneuropathy compatible with GBS.

Conclusion: We present a case of GBS in a setting of newly diagnosed Hodgkin's Disease. While causation cannot be implied, a similar association has been reported previously in patients with established Hodgkin's Disease.

\section{3-03}

\section{Segmental Zoster Paresis: A Case Report with Unusual} Electrophysiologic Features

\section{Grant, A. Purdy, T. Benstead (Halifax, Nova Scotia)}

Background: Neurogenic weakness follows herpes zoster infection in $3-5 \%$ of cases. Described electrophysiologic findings include low amplitude or absent sensory nerve action potentials (SNAPs) and compound muscle action potentials (CMAPs), and fibrillation potentials or positive sharp waves in involved myotomes. Complex repetitive discharges (CRDs) and myokymia have not been reported.

Methods: A 73 year old woman presented with proximal left upper extremity pain followed by a vesicular rash involving the C5-C7 dermatomes. Weakness of C5-C7 greater than C8-T1 innervated muscles began one week after the rash. Median and ulnar motor and antidromic sensory nerve conduction studies and concentric needle EMG were performed 7 weeks after onset of weakness.

Results: Left median CMAP and SNAP amplitudes were reduced; the left ulnar CMAP amplitude was borderline. Median motor conduction velocity was slow, without dispersion or block. Needle examination revealed abundant CRDs and less prominent myokymic discharges in C5-C7 limb muscles and cervical paraspinals. Poor activation of long duration, complex motor unit potentials and fibrillation potentials were present in a similar distribution. Infrequent fasciculation potentials were present in a C8-T1 distribution.

Conclusions: CRDs and myokymic discharges may accompany evidence of motor and sensory axon loss in segmental zoster paresis. CRDs are an unusual finding in subacute/early chronic radiculopathies. The presence of myokymia suggests hyperexcitability of motor axons; paraspinal involvement implies a proximal site, probably at the level of spinal nerve or ventral root.

\section{3-04}

Ataxia with Isolated Vitamin E Deficiency (AVED) in a 7year-old Girl. Case History with Videotape

\author{
R.L.L. Smith, J.T.R. Clarke, J. Vajsar (Toronto, Ontario) \\ Background: Ataxia with vitamin E deficiency (AVED) is
}

isolated to $8 \mathrm{q} 13$ and is caused by a mutation in the alpha tocopherol transport protein gene which affects vitamin $E$ incorporation into liposomes. It presents with a phenotype very like Friedreich ataxia. Serum vitamin E concentration may be reduced in both proband and carriers.

Method: A single case report with videotaped examination.

Results: An 7-year-old only child from a non-consanguineous German family presented with worsening gait ataxia and areflexia and a presumptive diagnosis of peripheral neuropathy. NCVs were consistent with an axonal sensory neuropathy. MRI showed a low conus initially suggestive of tethering. Clinical findings included esotropia, cerebellar dysarthria, mild muscle wasting with generalized hypotonia and $4 / 5$ distal weakness. She had absent DTRs with normal plantar responses and reduced vibration perception in the lower limbs. She had prominent axial, appendicular and gait ataxia. There was no clinical malabsorption. FRDA DNA studies showed no repeat expansions. Serum vitamin $\mathrm{E}$ was markedly reduced $(1.7 \mathrm{micromol} / \mathrm{L}$ : NORMAL $>12.0$ micromol/L) and she commenced supplementation in November 1997. AVED DNA studies are pending.

Conclusions: AVED is rare, but must be considered in autosomal recessive/sporadic chronic ataxia particularly with FRDA phenotype and negative DNA studies.

VIDEOTAPE: Demonstrates the clinical findings described in the text with prominent gait ataxia.

\section{3-05}

Dorsal Ulnar Cutaneous Nerve Injury Following an In-line Skating Accident

\section{Poulin, M Veilleux (Montreal, Quebec)}

Neuropathy of the dorsal ulnar cutaneous branch is rarely described. The dorsal cutaneous branch of the ulnar nerve arises above the wrist and supplies portion of the dorsum of the hand and the 4 th and 5 th fingers. We report the case of a 26 year old woman referred to the EMG Laboratory for pain and numbness over the dorsum of the right hand extending to the 3rd, 4th and 5th digits, following a fall while in-line skating. The patient did not use wrist guards. She noticed a bruise over the dorsum of the right wrist after the fall but $\mathrm{X}$-rays of the wrist were normal. When seen in the EMG Laboratory two months later, neurological examination revealed hypoesthesia in the distribution described above. Nerve conduction studies revealed a moderately reduced right dorsal ulnar cutaneous nerve action potential amplitude as compared to the left side, and concentric needle examination of the first dorsal interosseous and abductor digiti minimi muscles was normal. Electrophysiological findings were compatible with an isolated damage to the right dorsal ulnar cutaneous nerve. Injury to this nerve could have been prevented by the use of wrist guards.

\section{3-06}

\section{Polyneuropathy in Moderate Alcohol Consumers}

ML. D'Amour, S. Brissette, J. Lavoie, RF. Butterworth (Montreal, Quebec) 
Background: The adverse health effect caused by the moderate consumption of alcohol is not well described in the literature. We know that severe consumption of alcohol can cause several diseases, including peripheral neuropathy. We decided to look for signs of peripheral neuropathy in moderate drinkers.

Methods: We were able to recruit 30 moderate drinkers, taking 2-6 drinks/day (60-90 gms alcohol/day) and 46 severe drinkers, consuming more than 6 drinks/day ( $>100$ gms alcohol/day) with no other causes of polyneuropathy. Twenty-six normal subjects of the same age groups served as controls. Nerve conduction velocities were evaluated in 7 nerves for each subject. Since vitamin deficiencies, and more particularly thiamine deficiency, are considered a possible etiology of alcoholic polyneuropathy, we looked at the thiamine status of these patients, measuring the effect of thiamin diphosphate on transketolase levels.

Results: Following ANOVA analysis and a Bonferroni test, statistical differences were found between the groups either in the median and ulnar motor nerves or in the median, ulnar and sural sensory nerves. Severe drinkers had greater nerve involvement than moderate drinkers, although the latter were also affected. When evaluating the thiamine status, abnormal TDP values were found in some subjects from both the moderate and severe groups. A statistical difference was found upon a student $t$-test for the ulnar, motor and sensory nerves of these subjects as compared to the other subjects of the 3 groups who had a normal TDP values.

Conclusion: Moderate alcohol drinkers (2-6 drinks/day) show some electromyographic signs of peripheral neuropathy. A few of them have a poor thiamine status and there is a possible relationship between nerve conduction slowing and thiamine deficiency.

\section{3-07}

\section{Median Neuropathy Complicating Fractures of the Distal Radius: A Prospective Study}

\section{BR Guppy, R Grondin, PR Bourque (Ottawa, Ontario)}

Background: An association between trauma and carpal tunnel syndrome (CTS) has been known, but the frequency of CTS following wrist fracture is uncertain. In this prospective study, wrist fracture patients were assessed clinically and electrophysiologically for a 3 month period after fracture for the development of CTS.

Method: 58 patients (male-22\%; female-78\%; mean age $=52$ yrs.) with an uncomplicated fracture of the distal radius were recruited from the hospital fracture clinic. Dominant hand fracture occurred in 38\% of cases. Concurrent blind and independent clinical and nerve conductions assessments were performed immediately after cast removal and at 3 months.

Results: At cast removal, the incidence of clinical CTS was $12.1 \%$ while no cases of CTS were found in the unfractured wrist. Nerve conduction studies found CTS in $25.9 \%$ of fractured wrists and $20.7 \%$ of unfractured wrists. CTS was found in $15.4 \%$ of fractured wrists and $0 \%$ of non-fractured wrists at 3 months clinically but on nerve conductions studies, $15.4 \%$ of fractured wrists and $17.9 \%$ of non-fractured wrists had CTS. Ten percent of cases had bilateral CTS. About $5 \%$ of patients developed clinical CTS between the initial and the 3 month assessment while $7.8 \%$ of the patients had clinical CTS resolve during this same period. About $20.5 \%$ of cases had electrodiagnostic CTS resolve at 3 months while 5\% of patients developed CTS in this same period.

Conclusions: The frequency of clinically silent electrodiagnostic CTS was surprisingly high at baseline, but the presence of wrist fracture may have produced symptoms leading to the identification of the neuropathy. Severe wrist pain and paresthesia appear to predict the development of CTS. Clinical CTS may occur in up to $12 \%$ of cases of distal radius fracture initially, and in $17 \%$ of cases at 3 months.

\subsection{8}

\section{Thoracic Outlet Syndrome: A Reality?}

\section{Jacques, J. Cifelli (Montreal, Quebec)}

Objective and Importance: Thoracic outlet syndrome (TOS) is an exclusion diagnosis and due to this fact it is difficult to assess. Investigational methods are of limited value and clinical examination is the key to success. The aim of this presentation is to present a TOS case and underline different treatments as well as different surgical approaches to this problem.

Clinical Presentation: 43-year-old female patient presented with past surgical history of transaxillary resection of the 1st rib on August 21, 1992. On September 30th, 1997 she underwent fibrous band resection via supra and infravicular approach. She has 7 year history of pain between the scapula, posterior aspect of the left arm, medial aspect of the forearm and the last two fingers. On physical examination, ulnar distribution motor and sensory deficits were present. Investigation shows $\mathrm{C} 7$ transverse process as well as 1st rib remnant.

Intervention: She underwent brachial plexus exploration through a subscapular posterior approach on October 24, 1997. At one month follow-up she was pain-free and had no functional limitations.

Conclusion: True TOS does occur. Thorough exclusionary workup remains the key to the dignosis. Structural abnormalities as well as electrical concomitants are well demonstrated. Operative procedures for TOS which do not get close to the spine may fail. Most abnormalities involve neural elements which require direct inspection and neurolysis..

\section{3-09}

\section{Quantitative Sensory Testing in Syringomyelia}

\section{S. F. Wilson, J. D. Stewart, G. Bertrand (Montreal, Quebec)}

Objective: To evaluate the usefulness and correlation of quantitative sensory testing (QST) in patients with syringomyelia.

Background Information: Suspended sensory loss is an important sign in syringomyelia, but clinical sensory testing for temperature is often difficult and uncertain. We evaluated the utility of a QST device in four patients with syringomyelia. 
Methods: The patients had radiologically proven syringomyelia. QST for warm and cool was performed using a Somedic thermal testing device. Two to six QST examinations were completed on the patients, and in three patients performed pre and post-operatively. The results were compared with the clinical examination.

Results: All patients had clear abnormalities on QST. The clinical examination showed similar defects. All showed improvement in QST over time and this correlated with a decrease in syrinx size on MRI

Conclusions: 1. QST was clearly abnormal in these patients who had clinical, thermal and pinprick abnormalities. 2. QST was more sensitive to loss of warm than cold sensation. 3. QST has the advantage of testing a range of temperatures and thus has the potential to detect subtle abnormalities. 4. The clinical examination has the advantage of quickly finding sensory levels.

\section{3-10}

Prevalence of Psychiatric Manifestations in Post-Operative Temporal Lobectomy Epileptic Patients

JB Richards, MA Lee (Calgary, Alberta)

Background: Since the inception of surgical intervention for intractable psychomotor seizures in epileptic patients, there has been the suggestion in the literature that post-operative temporal lobectomy (TLX) patients may experience a concomitant onset of psychiatric illness. The incidence of post-TLX psychiatric manifestation has been reported to be between 3.8 and $35.7 \%$, with a mean of $7.6 \%$. Psychiatric illnesses that have been reported include psychosis, depression and suicide. The purpose of this study has been to describe the incidence of psychiatric referral and diagnosis in post-TLX patients at the Foothills Hospital.

Methods: The objectives of this study have been met by a retrospective review of patients who have received TLX at the Foothills Hospital over the past ten years. The use of psychiatric medical charts has allowed us to discern the psychiatric diagnosis of each referred patient and further, to discover if there is a prevalence of any one psychiatric illness.

Results and Conclusions: The results of this study indicate that there are important and frequent post-operative psychiatric manifestations of TLX. In addition we can conclude that both pre and post-operative psychiatric evaluations of TLX patients are important in ensuring their psychiatric stability.

\section{3-11}

\section{Morphology Of Depth-Recorded Mesial Temporal Seizure Onsets}

Richard Wennberg (Toronto, Ontario), L. Felipe Quesney, André Olivier (Montreal, Quebec), Francisco Arruda (Riberrâo Preto, Brazil)

Background: The electrographic morphology of mesial temporal seizure onsets was examined in 18 patients with temporal lobe epilepsy.
Methods: Three bilateral depth electrodes, inserted stereotactically through the second temporal gyrus, recorded from amygdala (AM), anterior hippocampus $(\mathrm{H})$ and parahippocampal gyrus $(\mathrm{PH})$. Ictal onset patterns for all focal ( $\leq 2$ contacts of one electrode) and regional ( $>2$ contacts of one or more adjacent electrodes) onset seizures were classified into four groups: rhythmic limbic spiking $<2 \mathrm{~Hz}$ (RLS), spike-and-wave activity $>2 \mathrm{~Hz}(\mathrm{~S} / \mathrm{W})$, rhythmic sharp activity $<13 \mathrm{~Hz}(\mathrm{RS})$ and rhythmic polyspike activity $>13 \mathrm{~Hz}$ (RPS).

Results: Of 321 total seizures onsets were: $50 \%$ RPS; $35 \%$ RS; $11 \%$ RLS; $4 \%$ S/W. Focal onsets more often showed RLS than regional onsets $(22 \%$ vs. $5 \%$ ) while RPS was more common in regional than focal onsets $(59 \%$ vs. $37 \% ; p<0.001)$. Nine patients had $\geq 2$ different patterns of ictal onset. Focal $A M$ and $H$ onsets did not differ but RLS was not recorded in focal PH seizures.

Conclusions: There is no ictal electrographic pattern specific for a given limbic localization. RLS can be recorded in focal AM onsets and is thus not dependent on intrahippocampal pathoanatomy. RPS is more common in regional than focal onsets.

\section{3-12}

\section{Somatosensory Potentials in Juvenile Myoclonic Epilepsy}

DR McLean, B Stigsby, S Bohlega (Riyadh, Saudi Arabia)

Background: Salas-Puig et al reported "giant" somatosensory potentials (SEP) in $14 \%$ of patients with juvenile myoclonic epilepsy (JME). As giant potentials in JME is not our experience and is not reported elsewhere, we attempted to verify the observation.

Method: The SEP were analyzed prospectively in twenty four JME patients seen between 1995 and 1997. The SEP was obtained using standard stimulating and recording techniques. The latencies of N19 \& P22 (median), N35 \& P37 (tibial) and the peak to peak amplitude of N19/P22, P22/N30 and N35/P37, $\mathrm{P} 37 / \mathrm{N} 45$ were analyzed and compared with controls using the Student's t-test.

Results: Of 24 patients, only one had an abnormally high N19/P22 and P22/N30 amplitude. There was no difference in the mean amplitude between JME patients and controls. The absolute latency of the various waves were normal. The single patient with a high amplitude SEP had no unique identifiable clinical or EEG characteristic except that she was on carbamazepine and the others received sodium valproate. She had no clinical features of progressive myoclonic epilepsy.

Conclusion: We were able to confirm a high SEP in JME but the frequency was lower than previously reported ( $4 \%$ vs $14 \%$ ).

\section{3-14}

\section{Correlative Studies Of Subdural Electroencephalography}

Warren T. Blume, Giannina Holloway (London, Ontario)

Aims: Value of scalp recorded seizures and subdural interictal 
spikes in predicting origin of subdural recorded temporal lobe seizures was sought.

Methods: Seven-contact lines were inserted to cover inferior mesial and lateral temporal regions. Neocortical convexity lines were placed in all 26 patients.

Results: Most seizures of 25/26 patients arose from the side of ultimate lobectomy. Of 25 patients with adequate mesial and lateral coverage, seizure origins were: ipsilateral mesial temporal (22 patients), ipsilateral lateral temporal ( 2 patients), ipsilateral mesial and contralateral lateral temporal (1). 221 (78\%) of 285 total clinical seizures arose from the ipsilateral mesial temporal region, $11(4 \%)$ arose from the contralateral mesial temporal area, $26(9 \%)$ from the ipsilateral lateral temporal region, and $9(3 \%)$ from the contralateral lateral temporal region. Among 15 patients with both scalp and subdural-recorded seizures, those of the scalp arose ipsilaterally in $11(73 \%)$. Most active cortical spikes appeared in the same gyrus as most seizures in $19(73 \%)$ of 26 patients, appeared in a different gyrus of the same lobe in $5(19 \%)$, and occurred contralaterally in $2(8 \%)$.

Conclusions: Among patients with inconclusive scalp EEG data we conclude: 1) lateralisation of scalp recorded seizures cannot be ignored in this assessment, 2) most subdural seizures arise from the mesial temporal region, and 3) most active interictal subdural spikes correlate with seizure origin.

\section{3-15}

\section{Propagation of Frontal Lobe Seizures: A Scalp EEG Study}

Warren T. Blume, Veena Kander (London, Ontario)

Aims: As semiology of frontal lobe seizures depends heavily upon propagation of ictal discharges, its patterns were sought.

Methods: Forty-eight seizures of 35 patients were visually subdivided into phases defined as distinct ictal morphologies.

Results: Surprisingly, no propagation occurred during the initial phase transition (Phase I to II) in 28/48 (58\%); ipsilateral propagation occurred in 12/48 (25\%); secondary bisynchrony appeared in $5 / 48(10 \%)$; homotopic spread characterised $2(4 \%)$ of seizures; spread was restricted to the frontal sagittal region in $1(2 \%)$.

Conclusions: The infrequency of homotopic and generalised initial spread of frontal seizures (15\%) suggests that early clinical features likely reflect side of seizure origin.

\section{3-16}

Ear Clicking in Symptomatic Palatal Tremor: Its Origin and Treatment with Botulinum Toxin A

A-L Lafontaine, W. Murphy, O. Suchowersky, R. Lee (Calgary, Alberta)

Palatal tremor can be classified into two distinct entities: essential palatal tremor (EPT) and symptomatic palatal tremor (SPT). EPT has no known etiology, whereas SPT has clinical and radiological evidence of underlying brainstem or cerebellar pathology. It has been reported that ear clicking is exclusive to EPT. The origin of ear clicking remains controversial.
Botulinum toxin (Botox) has been reported to successfully treat ear clicking associated with palatal tremor in a total of 7 patients to date.

A 42 year old female developed sudden onset of ear clicking, associated with involuntary movements of the palate in October 1996. An MRI revealed a left cerebellar cavernoma. The clicking sound was recorded by a microphone placed in the ear. Needle EMG was performed in both the tensor veli palatini and the levator veli palatini. The tensor veli palatini was found to be inactive, while there were bursts of activity arising from the levator veli palatini that were synchronized to the ear clicks. $15 \mathrm{U}$ of Botox was injected in the activated levator veli palatini.

These findings suggest that ear clicking cannot reliably distinguish EPT from SPT. We also provide further evidence that ear clicking can arise from activation of the levator veli palatini. The response to the treatment with Botox is currently being assessed.

\section{3-17}

\section{Propriospinal Myoclonus Following Dorsal Pontine Hemorrhage}

S. Furtado, A-L Lafontaine, R. Ranawaya, D. Patry, A.M. Buchan (Calgary, Alberta)

This 38 year old man sustained a hypertensive dorsal pontine hemorrhage. Months later, he began to show brief, rapid extension movements of his back, with hip flexion and knee extension, occurring several times per minute. He can neither predict, nor prevent the movements, which occur in all positions; they are irregular, spontaneous, and not stimulus sensitive.

MR brain showed dorsal pons hypodensity, consistent with old pontine hemorrhage. MR cord was attempted, but not completed. Spontaneous EMG activity showed position-independent lower rectus abdominus activation prior to upper rectus abdominus, cervical sternocleidomastoid, quadriceps, or paraspinal activation. Thus, EMG showed the abnormal movements are essentially flexor in nature; the appearance of axial extension is likely a reflex action. Routine EEG was normal. Myoclonus was not associated with cerebral activity.

The movement disorder in this patient may represent propriospinal myoclonus, given the EMG activation pattern and the normal EEG. This is a type of spinal myoclonus where the abnormal neural activity may arise in the mid-thoracic cord. The movements typically consist of axial flexion movements that are brief, irregular, spontaneous, and symmetric. EMG findings in propriospinal myoclonus are similar to those found here. Pathology, when present, has typically involved the cervical cord, not the brainstem, thus making this patient unusual in the presumed location of pathology.

\section{3-18}

L-Carnitine Supplementation In Hyperammonemic Adults Taking Valproic Acid

D. A. DeRubeis, G. B. Young, T. Rupar (London, Ontario)

The benefits of carnitine supplementation in children with 
valproic acid (VPA) induced hyperammonemia are documented in the literature. We report the metabolic and clinical effects of carnitine supplementation in two mentally subnormal epileptic adults taking VPA, who had low plasma carnitine, reduced free carnitine / acyl carnitine ratios and elevated ammonia levels.

Each patient served as his / her own control and received $3 \mathrm{~g}$ of carnitine per day for 6 weeks.

Plasma total carnitine, free carnitine, acyl carnitine, ammonia and VPA levels were measured prior to commencing carnitine, 3 weeks after reaching the target dose of $3 \mathrm{~g} /$ day and 6 weeks after discontinuing carnitine. Care givers provided logs of sleep time. Objective timed motor tasks and videotaped gait analysis were performed at each visit.

Carnitine supplementation was associated with decrease in sleep time, improvement in gait and performance in timed motor tasks. There was no change in seizure frequency or VPA levels. Ammonia levels were decreased. Plasma total carnitine, free carnitine and acyl carnitine / free carnitine ratios were increased.

Withdrawal of carnitine supplementation was associated with an increase in sleep time, deterioration of gait and slowing of motor tasks. Metabolic parameters returned to pre-carnitine supplementation values.

A large study is required to determine whether carnitine supplementation in hyperammonemic adults consistently improves clinical and biochemical parameters.

\section{3-19}

Chronic Periodic Lateralized Epileptiform Discharges (PLEDs) During Sleep Following Ventral Lateral Thalamotomy: Insights into the Anatomical Circuitry of PLEDs

\section{DW Gross, LF Quesney, AF Sadikot (Montreal, Quebec)}

Background: PLEDs have been recognized for 33 years; however, little is known about the underlying mechanism causing periodic discharges. The following case provides an opportunity to study PLEDs in a patient with precisely localized subcortical grey matter lesions.

Methods: Routine EEGs and overnight polysomnography were performed on the study patient. Standard 10-20 electrode positions were used, as well as EOG and chin EMG for polysomnography.

Results: The study patient was a 39 year old woman with right hemi-dystonia. She had left ventral-lateral (VL) thalamotomies in 1989 and 1991, left pallidotomy in 1992, and left centromedian thalamic stimulator implantation in 1997. One month after CM nucleus stimulatory implantation, the patient had awake EEGs and polysomnography. EEG during wakefulness and REM sleep was normal. With synchronized sleep (stages IIIV) left hemisphere PLEDs at $1-2 \mathrm{~Hz}$ were seen with fronto-centro-temporal predominance. PLEDs disappeared with arousal. Sleep spindles were seen in bilaterally. EEG with sleep in 1991 showed similar results. There was no history of seizures, before or after surgery.

Conclusions: PLEDs during synchronized sleep in a patient with thalamic lesions suggest that periodic phenomenon can be generated by an abnormal response of the thalamocortical neurons to rhythmic burst firing by the reticular thalamic nucleus.

\section{Reflex Epilepsy Induced by the Tower of London Task}

D.W. Gross, F. Andermann, F. Dubeau, G.M. Remillard (Montreal, Quebec)

Background: Seizures induced by cognitive tasks are well recognized. Activation of interictal epileptic abnormalities, and the sensitivity of patients to various tasks is variable. Controversy exists as to whether reflex epilepsy activated by intellectual activities represents focal or generalized epilepsy. Previous reports have demonstrated the importance of parietal tasks in seizure activation.

Methods: A 21 year old man with a history of generalized convulsions induced by intellectual tasks was investigated with EEG/ video telemetry, neuroimaging and a wide range of neuropsychological tests.

Results: MRI was normal. Interictal EEG showed symmetrical bifrontal epileptic discharges which were not activated by tasks. Two generalized seizures were recorded. One occurred while the patient was playing cards and one while doing the Tower of London task (TOL). Ictal EEG showed bifrontal polyspike discharges at seizure onset. On full doses of medication the TOL did not induce a seizure.

Conclusions: Interictal and ictal EEG suggested a generalized epileptic disorder. The activating task in this patient resembles epilepsy induced by "Soroban" calculation (Yamamoto et al). Spatial planning (a frontal lobe function) is central to the TOL. Along with card playing (parietal), frontal lobe activation (TOL) induced seizures in this patient.

\section{3-21}

Perinatal Acute Total Hypoxic-Ischaemic Encephalopathy (ATHIE): Clinical, Neuroradiological and Pathological Features

S.D. Levin, C. Illiffe, D.H. Lee, D.A. Ramsay (London, Ontario)

Background: Most cases of perinatal HIE are considered to be due to prolonged partial hypoxia-ischaemia. ATHIE is described rarely.

Objective: To describe the clinical, neuroradiological and pathological findings of perinatal ATHIE.

Method: Patients with perinatal HIE with features atypical for PPHIE (little brain-swelling, little multi-organ involvement, persistent brainstem abnormalities) were reviewed.

Results: 7 infants fulfilled these criteria. 4 died 3 of whom had autopsies. The patients had the following common characteristics: normal pregnancy, gestation 38-4I weeks, mean birth weight $4.43 \mathrm{~kg}$., complicated deliveries ( 3 with cord prolapse, I with shoulder dystocia and 1 with late foetal heart rate decelerations) with obstetric intervention ( 3 emergency caesarian sections, 3 forceps). Apgar scores were $0-1$ at 1 minute, $0-4$ at 5 
minutes, 0-4 at 10 minutes and 2-5 at 15 minutes. No patients had evidence of raised intracranial pressure and all survivors had persistent brainstem abnormalities. Neuro-imaging (CT and/or MRI) showed bilateral thalamic and/or basal ganglia infarction. All survivors showed a similar pattern of dystonic bilateral hemiparesis and relatively poor head growth. Pathological findings demonstrated brainstem, basal ganglia or hippocampal infarction or ulegyria.

Conclusions: ATHIE is a relatively uncommon syndrome of perinatal HIE but may represent an important subgroup of unavoidable obstetric disasters.

\section{ONCOLOGY}

\section{4-01}

\section{Alterations in the rap1 Signaling Pathway are Common in Gliomas but Not in Other Nervous System Tumors}

D. H. Gutmann (St. Louis, Missouri), J. E. DeClue (Bethesda, Maryland), A. Guha (Toronto, Ontario)

Several inherited predisposition to cancer syndromes are associated with the development of nervous system tumors. Tuberous sclerosis complex (TSC) is an autosomal dominant disorder in which affected individuals develop astrocytomas. One of the genes responsible for this disorder is TSC2 located on chromosome $16 \mathrm{p}$, encoding a $180 \mathrm{kDa}$ protein (tuberin) that functions in part as a negative regulator of rap1. Previous studies from our laboratory demonstrated that $30-50 \%$ of sporadic astrocytomas have reduced or absent TSC2 expression. In addition to loss of tuberin in sporadic astrocytomas, aberrant rapl mediated signaling may also be a result of overexpression of rapl. In this study, we test the hypothesis that alterations in the rapl signaling pathway are frequently observed in certain subsets of gliomas, compared to other tumors of the nervous system. Analysis of sporadic malignant astrocytomas and ependymomas demonstrated either increased rapl or reduced/absent tuberin protein expression in 50-60\% of different cohorts of these gliomas, compared to $30-33 \%$ of sporadic neuroblastomas, schwannomas and meningiomas and none of eight oligodendrocyte tumors. These results suggest that alterations in the rap 1 signaling pathway are important in the development of certain sporadic human gliomas.

\section{4-02}

\section{Platelet Derived Growth Factor Receptor Activation in} Human Meningioma Specimens

S. Shamah, J. Alberta, P. McLBlack, R. Carroll, C. Stiles (Boston, Massachusetts), A. Guha (Toronto, Ontario)

The $\beta$ receptor subunit of platelet-derived growth factor (PDGF) and its corresponding ligand (PDGF-BB) are coordinately expressed in fresh surgical isolates of human meningioma. These observations imply that PDGF autocrine loops are engaged in human meningioma and suggest that activated PDGF $\beta$ receptors might contribute to the pathology of this common brain neoplasm. However, the study of PDGF autocrine loops in human meningiomas has been slowed by the scarcity of meningioma cell culture model systems. Furthermore, in meningioma tumor tissue the activation state of PDGF receptors is difficult to assess with conventional reagents because the tumor is intermixed with normal stroma. In fact, there is no evidence that PDGF receptors within the tumor are activated by ligand. In this study we examined the activation status of the PDGF $\beta$ receptor in surgical meningioma specimens.

We made a synthetic tyrosine phosphopeptide to raise an antibody that reports the phosphorylation state of tyrosine 751 in the human PDGF $\beta$ receptor. Phosphorylated tyrosine 751 is a recognition site for phosphatidylinositol 3'kinase (PI3 kinase), a cytoplasmic effector of PDGF induced mitogenesis, chemotaxis, and membrane ruffling.

The activation specific antibody only recognized the tyrosine-751 binding site specifically on the PDGF $\beta$ receptor. Immunoblotting and immunostaining analyses with the activation specific antibody show that the PDGF $\beta$ receptor is constitutively phosphorylated at tyrosine 751 within multiple fresh surgical isolates of human fibroblastic and transitional meningiomas. These findings are consistent with a role for activated PDGF $\beta$ receptors, and more specifically the PI3 kinase signalling pathway in the proliferation of human meningiomas.

Activation specific antibodies, such as the one developed for the PDGF $\beta$ receptor, allows a molecular classification of tumors including those in the nervous system. They give us information not only regarding which growth factor receptors are activated, but also which intracellular signalling pathway(s) are potentially contributing towards tumor growth. As potential biological therapies targetted towards specific signalling molecules are developed, molecular classification with these kinds of reagents will be important to tailor these therapies to individual tumors.

\section{4-03}

A Prospective Trial of Awake Craniotomy as the Routine Surgical Approach to Supratentorial Intra-axial Tumours

\section{M.D. Taylor, M. Bernstein (Toronto, Ontario)}

Background: In the past, awake craniotomy has been used sporadically to permit brain mapping for epilepsy surgery and for excision of supratentorial mass lesions in areas of eloquent cortex. We propose that awake craniotomy is an excellent approach for most supratentorial intra-axial tumours.

Methods: A prospective trial was undertaken of 200 patients with supratentorial intra-axial tumours operated on under local anesthetic by a single neurosurgeon at a single institution. Clinical presentation, comorbid conditions, tumour location and histology, complications, and resource utilization were recorded.

Results: There were 121 gliomas, 63 metastases, and 16 miscellaneous lesions. Complications were seen in $33 / 200$ patients (16.5\%) with 2 deaths ( $1 \%$ mortality rate). New post-operative neurological deficits were seen in $13 \%$ of patients but were permanent in only $4.5 \%$. Of the most recent 50 patients treated, an 
ICU bed was used in $3(6 \%)$ and the median total hospital stay was I night.

Conclusions: Awake craniotomy is an excellent alternative to craniotomy under general anesthesia for supratentorial intraaxial tumours as it allows brain mapping, avoids general anesthesia, has a low medical and neurological complication rate, and minimizes resource utilization.

\section{4-04}

\section{Pleomorphic Xanthoastrocytoma}

PJ Muller, J Bilbao (Toronto, Ontario)

Pleomorphic xanthoastrocytoma is an astrocytic tumor variant that has been identified in the last two decades. Russell and Rubinstein' note the importance of identifying this tumor since there is a disparity between the ominous looking histology and a usually favorable postoperative course. They note that this tumor has been variously referred to as a superficially situated anaplastic pilocytic astrocytoma, giant cell glioblastoma, atypical fibroxanthoma or xanthosarcoma. These tumors have tended to be found in young patients [second decade most frequent], have an equal gender distribution, a predilection for the temporal lobe, a tendency to present with a long history of seizures and a very favorable clinical behavior post operatively. However, they may recur with or without a malignant degeneration.

We are presenting two patients, aged 54 and 67 , both male with an occipital and a frontal pleomorphic xanthoastrocytoma, respectively. Both underwent aggressive but subtotal tumor resection; the older also had post-operative radiation therapy. Both have had no deterioration in their neurologic state to date.

Although pleomorphic xanthoastrocytomas generally occur in a young age group they may occur in middle age and older patients.

1. Russell D and Rubinstein L: Pathology of Tumours of the Nervous System. Williams and Wilkins, Baltimore, 5th edition, 1989 pp 120-121

\section{4-05}

Primitive Neuroectodermal Tumour in a Patient with Naevoid Basal Cell Carcinoma Syndrome: Case Report

\section{PD McNeely, WJ Howes, DB Clarke (Halifax, Nova Scotia)}

Background: Naevoid basal cell carcinoma (NBCC) syndrome, also known as Gorlin's syndrome, is an autosomal dominant disorder which has been localized to chromosome $9 \mathrm{q}$. The hallmarks of this disorder include multiple basal cell carcinomas, jaw cysts, palmar or plantar pits, calcification of the falx cerebri, and rib abnormalities. Patients who have this syndrome are also at increased risk of developing primitive neuro-ectodermal tumours (PNET) of the cerebellum.

Methods: A review of the literature was performed. The patient's history, physical examination, and investigations are described.

Results: A five year old boy with NBCC syndrome presented with a two week history of intermittent vomiting, headache, and ataxia. Family history was positive for NBCC syndrome in two first degree relatives. Physical examination was remarkable for macrocrania, hypertelorism, papilloedema, and unsteady gait. Magnetic resonance imaging (MRI) revealed a large fourth ventricular tumour, and metastases to the spinal cord and pineal gland. Pathology confirmed the diagnosis of PNET.

Conclusions: This case illustrates the association between NBCC syndrome and PNET. Given the significantly increased risk of PNET in patients who have this syndrome, current recommendations are careful neurological examination every 6 months and yearly intracranial imaging for paediatric patients who have a first degree relative with NBCC syndrome.

\section{4-06}

Preoperative Chemotherapy for Choroid Plexus Carcinoma: A Case Report

\section{S.P. McDonald, K.E. Aronyk, (Edmonton, Alberta)}

Background: Choroid plexus carcinoma is a rare tumor of childhood and is associated with a poor prognosis for long-term survival. Gross total resection offers the greatest chance for prolonged survival or cure. Efforts at complete surgical resection are complicated by the highly vascular nature of the tumor and its usually large size. Preoperative chemotherapy following POG protocol 9233 results in considerable reduction in tumor size and vascularity making a second stage operation safer and more successful.

Method: A 29-month old boy presented to our institution with decreased level of consciousness and left hemiparesis. Imaging studies demonstrated a large, lobulated mass in the right lateral ventricle. Attempt at surgical resection was aborted intraoperatively due to extensive blood loss. A biopsy was consistent with a choroid plexus carcinoma.

Results: The patient underwent six successive courses of chemotherapy following POG protocol 9233 (vincristine $0.065 \mathrm{mg} / \mathrm{kg} / \mathrm{dose}$; cyclophosphamide $65 \mathrm{mg} / \mathrm{kg} / \mathrm{dose}$; VP-16 $6.5 \mathrm{mg} / \mathrm{kg} / \mathrm{dose}$; cis-platinum $4.0-5.0 \mathrm{mg} / \mathrm{kg} / \mathrm{dose}$ ). Follow-up MRI studies confirmed significant reduction in tumor volume making a second stage operative procedure possible.

Conclusions: Preoperative chemotherapy for choroid plexus carcinoma is effective in reduction of tumor volume and vascularity making a second stage operation safer and more successful.

\section{4-07}

Management Outcome of Benign and Aggressive Fibromatoses of the Head and Neck

F. DeMonte (Houston, Texas), M.J. Perez-Cruet (San Antonio, Texas), R.S. Weber (Philadelphia, Pennsylvania)

Background: Fibromatoses are uncommon, non-metastasizing fibrous tumors of unknown pathogenesis. A number of 
characteristics including their local invasiveness, recurrence after surgical excision, and occurrence at multiple sites makes these tumors difficult to treat effectively. Superficial fibromatoses tend to run a benign course while deep fibromatoses (desmoids) have a more rapid growth and aggressive behavior.

Methods: A retrospective analysis of the pathology, treatment, and outcome of patients having either benign or aggressive fibromatoses was conducted. A search of our institutional database identified 36 patients with benign (fibroma or fibromyxoma) and 54 patients with aggressive (demoids or fibrosarcoma grade 1) fibromatoses.

Results: Of the 36 patients with benign lesions (14 males, 22 females; median age 49 years, range $1-79$ years), 30 had surgical resection, 5 were not treated and 1 was given chemotherapy. Thirty-four patients remain alive with a mean follow-up of 3.7 years. The 2 deaths were not related to tumor recurrence. The 54 patients with aggressive tumors ( 30 males, 24 females; median age 34 years, range 1-88 years) underwent many different treatments including surgery (23 patients), radiotherapy (3), chemotherapy (3) and combination of surgery and radiotherapy (14), surgery and chemotherapy (7), and surgery, radiotherapy and chemotherapy (1). Three patients were not treated. Fortythree patients are alive after a mean follow-up of 8.3 years. Five of the eleven patients $(45 \%)$ who died were not treated or treated with only a single modality.

Conclusions: Benign fibromatoses are effectively managed with surgery alone. Multimodality therapy has improved the outcome of patients with aggressive tumors.

\section{4-08}

\section{Primary Tumors of the Sphenoid Sinus}

\section{F. DeMonte, G.L. Clayman, L. Ginsberg (Houston, Texas)}

Background: Neoplasms of the paranasal sinuses account for $0.2-0.8 \%$ of all cancers and for $2-3 \%$ of head and neck cancers. Primary involvement of the sphenoid sinus occurs in only $1-2 \%$. Tumors at this site commonly present with cranial neuropathies and require multidisciplinary treatment.

Methods: Over a 21 year period 27 patients with primary sphenoid sinus tumors were evaluated. Twenty-four subsequently received treatment for their neoplasm. The records of all patients were reviewed for the following parameters: age, sex, and race; presenting symptoms and signs; radiographic features and sites of tumor extension; pathology; prior treatments; surgical approaches and extent of resection; adjuvant therapy; complications and patient outcome.

Results: A malignant pathology was encountered in 26 of these 27 patients. Pituitary tumors were excluded. The most common pathologies were SCC (9), adenoid cystic carcinoma (4), chondrosarcoma (3), and neuroendocrine carcinoma (3). Treatments included surgery (1), surgery and radiotherapy (XRT) (6), surgery and chemotherapy (3), surgery, XRT, and chemotherapy (4), chemotherapy (5), chemotherapy and XRT (3) and XRT (2). At last follow-up 14 patients were still alive $(56 \%)$ while 11 have died $(44 \%)$.

Conclusion: Nearly all of these patients had malignant tumors of the sphenoid sinus. Treatment consisted of aggressive multimodality therapy. Use of skull base surgical techniques allowed for aggressive resection of some of these tumors. At last follow-up the majority of patients are alive. The patients' presenting symptoms and signs, operative management, and use of adjunctive therapies will be discussed.

\section{4-09}

\section{Outcome Following Orbital Entry During Cranial Base Surgery}

F. DeMonte, D.L. Callender, J. R. Patrinely (Houston, Texas)

Background: Controversy exists regarding the need for and extent of orbital reconstruction necessary following orbital entry.

Methods: To clarify this issue a review of 71 patients operated upon between 11/92 and 3/97 in whom orbital entry was required was undertaken.

Results: 71 patients underwent 72 operations. 14 orbitectomies were performed and are excluded. As a sequela of the 57 operations in 56 patients 13 complications occurred. Of these 13 adverse outcomes 3 were transient complaints of diplopia. Persistent morbidity occurred in 10 patients although only 2 required/desired revision surgery. In all cases of enophthalmos/dystopia $2 / 3$ or more of the inferior wall (floor) was resected, usually in combination with $2 / 3$ or more of the lateral wall. Some form of orbital reconstruction was performed in 20/57 operations.

Conclusions: Patients undergoing inferior orbital wall resection, especially in combination with resection of the lateral wall are at increased risk of enophthalmos/dystopia. Primary reconstruction is recommended. In most patients elaborate reconstructive efforts are not necessary. The need for orbital resection/dissection should not be a deterrent to complete tumor removal.

\section{4-10}

\section{Deliberate Transsphenoidal Two-Staged Removal of Large And Giant Pituitary Adenomas}

\section{Lacroix, G. Mohr, J. Hardy (Montreal, Quebec)}

Introduction: Most pituitary macroadenomas with large and giant midline symmetrical suprasellar extension (SSE) can be successfully removed via the transsphenoidal approach. In some cases however, it is impossible to obtain the detachment of the SSE from the diaphragma sellae and further manipulation may be deleterious to optic structures, carotid arteries or hypothalamus. Thus a subtotal removal is carried out at first approach. The second session is planned deliberately as a gradual downward progression of the SSE into the sella on sequential postoperative imaging studies is observed. In such cases, a second operation is done via the same transsphenoidal route, thus avoiding a transcranial procedure.

Method: Since we first described this technique in 1982, we have encountered 25 cases in which a two staged removal was carried out. The group was constituted of 5 women and 20 men 
with a mean age of 46 years ( 22 to 77 years). Patients were followed-up for 1 to 14 years (mean 6.3 years).

Results: Two-staged surgery was deliberately planned in 22 cases, with a median interval of 3.8 months ( 1 to 18 months) between the two procedures. In three cases however, post-op hemorrhage forced us to intervene acutely and the residual SSE became mobile and could be removed. In 17/25 cases, complete removal of the SSE was attained after the second surgery. A craniotomy had to be performed in 3 cases to complete the resection. A complete course of radiation therapy (5000 rads) was given to 10 patients. The complications were: 2 panhypopituitarism, 5 fistula, 2 meningitis and 1 abscess. There were 2 recurrences.

Discussion: We recommend a planned two-staged transsphenoidal removal of large and giant SSE: besides reducing the risk of damage to the optic structures, the gradual descent of the large SSE may also decrease the risk of secondary hypothalamic prolapse into the emptied sella turcica and avoid the intrasellar herniation of the optic nerves.

\section{4-11}

\section{A Randomized Study of Palliative Radiation Therapy for Older Patients with Glioblastoma Multiforme}

D. Fulton, W. Roa, E. Bruera, J. Hanson (Edmonton, Alberta), G. Bauman, B. Fisher (London, Ontario), A. Chan, P. Forsyth, J. MacKinnon, G. Pelletier (Calgary, Alberta)

Background: Glioblastoma multiforme comprises the majority of primary brain tumors among the elderly. However, the prognosis of this group of patients is notoriously poor. For patients with a life expectancy of less than six months, a radical course of radiotherapy lasting six weeks accounts for a disproportionate amount of their estimated survival time. Alternative radiotherapy schedules might yield equivalent palliative benefits, less toxicity, shorter overall treatment times and less consumption of limited radiotherapy resources.

Methods: In this study, 28 patients older than 65 years were randomized following surgery to either standard radiotherapy ( 6000 cGy in 30 fractions over 6 weeks) or short course radiotherapy ( $4000 \mathrm{cGy}$ in 15 fractions over 3 weeks).

Results: The median survival for all patients was 26.1 weeks, 28.6 weeks for standard radiotherapy and 23.9 weeks for short course radiotherapy (NS, $\mathrm{p}=0.4$ ). Patients were seen one month after radiotherapy and every three months thereafter. There were no significant differences in Karnofsky Performance Status or quality of life as measured by the Spitzer Quality of Life scale between the two groups during follow-up.

Conclusions: We conclude that, so far, there are no disadvantages to treating this group of patients with a short course of radiotherapy and that advantages include a shorter radiotherapy treatment time for the patients and a lower cost of treatment.

\section{4-12}

\section{Human Glioma Fas / Fas-Ligand Expression}

I.F. Parney, J.F. Elliott, M.A. Farr-Jones, K.C. Petruk (Edmonton, Alberta)

Fas (CD95) and its ligand (FasL) are critical in cell-mediated immune responses. Naïve $T$ cells express Fas, rendering them sensitive to Fas-ligand. Activated cytotoxic $T$ cells up regulate FasL which induces apoptosis in target cells by binding Fas. Therefore, Fas/FasL expression is important in glioma immunobiology. We assessed Fas/FasL in six human glioma cell lines (D54MG, U251, U373, Ed147.BT, Ed149.BT, Ed186.BT).

Cell surface Fas/FasL expression was assessed by flow cytometry. A FasL bioassay was developed by transducing murine lymphoma cells (JFE-114.1) with human Fas, rendering them FasL sensitive. Secreted FasL activity was assessed by recording JFE-1 14.1 metabolic activity (MTT assay) after $24 \mathrm{~h}$ incubation in glioma-conditioned media. Cell surface FasL activity was assayed using two-color flow cytometry (propidium iodide, anti-AnnexinV) to assess JFE-114.1 apoptosis after 30 min coincubation with glioma cells.

Flow cytometry staining was positive for Fas and negative for FasL in all cell lines. JFE-1 14.1 metabolic activity was not affected by incubation in glioma-conditioned media, suggesting FasL was not secreted. However, four cell lines (U251, U373, Ed149.BT, Ed186.BT) caused a significant (10.4 - 15.0\%) increase in apoptotic JFE-1 14.1 cells after 30 min coincubation, suggesting membrane-bound FasL activity in these cells.

Fas expression suggests that gliomas may be sensitive to FasL-mediated killing. However, membrane-bound FasL activity in 4/7 cell lines despite negative FasL flow cytometry is puzzling. It suggests "clandestine" glioma expression of membranebound FasL that can be quickly up regulated when cells come into contact with infiltrating FasL-sensitive cells. This may be another means to avoid immune surveillance.

\section{4-13}

B7.2 and GM-CSF but not IL12-Transduced Human Glioma Cells Synergize with Wild Type to Stimulate Autologous Antitumor Lymphocytes In Vitro

I.F. Parney, K. Kane, M.A. Farr-Jones, L.-J. Chang, K.C. Petruk (Edmonton, Alberta)

Cancer immunogene therapy is based on vaccination with radiated, autologous tumor cells transduced with immunostimulatory genes. To determine an optimal glioma immunogenetic strategy, we examined lymphocytes stimulated with autologous human glioma cells transduced with B7-2, GM-CSF, and/or IL12.

A human glioma-derived cell culture (Ed 147.BT) was transduced with B7-2, GM-CSF, and/or IL 12 using retroviral vectors. Autologous peripheral blood mononuclear cells (PBMC) were cocultured ( 8 days $\times 2$ ) with radiated (20 000 rad) gene-transduced tumor alone or combined radiated gene-transduced and wild type tumor. PBMC proliferation was determined by serial 
cell counts. PBMC phenotype was assessed by flow cytometry for CD4, CD8, and CD16. Antitumor cytotoxicity was determined by $51 \mathrm{Cr}$ release assay.

PBMC cell numbers all decreased during primary stimulation. Tumor cells expressing B7-2 or GM-CSF alone consistently caused secondary proliferation. Cells expressing more than one gene caused secondary proliferation when cultured without wild type cells. Tumors expressing more than one gene consistently increased PBMC percentages of CD8+ (cytotoxic T) and CD16+ (natural killer) lymphocytes. Interestingly, antitumor cytotoxicity only exceeded that of PBMC stimulated with wild type tumor alone when PBMC were stimulated with both wild type tumor and B7-2/GM-CSF- (but not IL12) transduced tumor.

PBMC proliferation and phenotype is altered as expected by exposure to therapeutic genes. However, gene-transduced tumor cells alone do not stimulate greater PBMC antitumor cytotoxicity than wild type tumor. Only B7-2/GM-CSF-transduced cells combined with wild type produced significantly increased cytotoxicity. This may reflect selection of tumor subclones with different antigenic spectra during retrovirus-mediated gene transfer.

\section{4-14}

Primary Low Grade Central Nervous System Lymphoma (PCNSL) in a Child - A Case Report

DA Steven, RF Del Maestro, DA Ramsay, DR Macdonald, K Rizkalla (London, Ontario)

Background: One to $2 \%$ of primary brain tumors are lymphomas (PCNSL) of which approximately $6 \%$ of reported cases occur in children. The majority of PCNSL are of intermediate or high grade(Working Formulation). A case of PCNSL is described that is unusual for its occurrence in a child and its "low grade" histological appearance.

Case Report: An otherwise healthy 4 year old girl presented with focal seizures following a mild head injury. CT and MRI examinations revealed a large, irregularly enhancing right parietal mass. A craniotomy with gross total resection of the neoplasm was carried out after two weeks of treatment with anticonvulsants and dexamethasone. Extensive investigations failed to reveal any evidence of extraneural lymphoma or immune suppression. The child is well 4 months after surgery with no radiological evidence of recurrence. No radiotherapy or chemotherapy was given.

Laboratory Observation: The sections showed a perivascular and intervascular cellular brain infiltrate of monomorphic lymphocytes characterised by minimal cytoplasm and small round nuclei with undulating margins and indistinct nucleoli. The atypical lymphocytic infiltrate was immunopositive for the B cell marker, CD20. No evidence of Epstein-Barr infection was found by EBV-LMP immunostain or in situ hybridization. Flow cytometry showed a diploid cell population composed of $70 \%$ B cells (70\% mature CD 19 and CD 20 positive cells) and $14 \% \mathrm{~T}$ cells with polyclonal $B$ and $T$ subsets. Gene rearrangement studies for Ig heavy chain, TCR-( and $\mathrm{Bcl}-2$ genes were negative.
Conclusion: Although the histological appearance of the tumour was essentially diagnostic of low grade lymphoma, atypical and inexplicable features included the low grade of the neoplasm, the lack of steroid-induced lympholysis and the failure to detect monoclonality by flow cytometry. Lymphomatoid granulomatosis, plasmacytoid granuloma, "inflammatory pseudotumor", Langerhans-cell histiocytosis, a non-specific parainfectious inflammatory disorder and a reaction to an underlying neoplasm formed part of the differential but were excluded, largely on histological grounds and confirmatory immunohistochemical reactions. The prognosis is uncertain. The plan is to follow the child closely by MRI scan and defer treatment until recurrence.

\section{4-15}

Familial Adenomatous Polyposis and Benign Intracranial Tumors: A New Variant of Gardner's Syndrome

\section{R Leblanc (Montreal, Quebec)}

Introduction: Familial adenomatous polyposis (FAP) is associated with malignant tumors of the central nervous system (Turcot's syndrome) and with cranial and facial osteomas (Gardner's syndrome). We report the occurrence of benign intracranial tumours in two individuals from the same family with Gardner's syndrome comprising a new, distinct clinicalpathological entity.

Patients and Methods: A 57 year old woman (the propositus), her sister, and two of her nieces were diagnosed with Gardner's syndrome. The propositus complained of vertigo from what proved to be a cerebello-pontine angle epidermoid cyst. Her children and other individuals in her family with Gardner's syndrome were examined and underwent enhanced, high-resolution CT scanning or enhanced MR imaging. One of her nieces with Gardner's syndrome, aged 39 years, was found to have a left frontal meningioma. Other members of the family seen to date do not have intracranial lesions.

Discussion: Familial adenomatous polyposis, Gardner's syndrome and Turcot's syndrome with medulloblastomas are linked to a mutation of the adenomatous polyposis coli (APC) gene, while Turcot's syndrome with glioblastoma multiforme is associated with mutation of a mismatch-repair gene. The demonstration that Gardner's syndrome can be associated with benign intracranial neoplasms (epidermoid cysts and meningiomas) in contradistinction to Turcot's syndrome which is associated with malignant intracranial neoplasm (glioblastoma multiforme and medulloblastoma) constitutes a new, extracolonic manifestation of FAP. That the lesions are benign adds to the incentive for elective CT or MRI screening of patients with FAP and Gardner's syndrome as these can be treated even more successfully than the malignant lesions associated with Turcot's syndrome. The molecular characterization of our patients should reveal if benign intracranial lesions represent a pleiotropic manifestation of the APC mutations associated with Gardner's syndrome or if other genes are implicated. 


\section{4-16}

\section{Multifocal Adult Medullablastoma Presenting as Chiari Malformation}

\section{P Ellis, R Kerr, J Rossitor, R Smith (Kingston, Ontario)}

We present the case of a 26 year old male with multifocal medulloblastoma and Chiari type I malformation. He presented with a 3 week exacerbation of a long history of headache in the frontal region and subocciput, which was worsened by valsalva and ameliorated by upright sitting position and applied pressure to the subocciput. The patient complained of morning nausea and vomiting and was noted to have a mild Parinaud's syndrome. MRI demonstrated lesions that were low intensity on T1, high on T2 and minimally enhancing with gadolinium. These were located in the right external capsule and in left cerebellar tonsil. Both cerebellar tonsils were herniated $8 \mathrm{~mm}$ below the foramen magnum. The patient was treated with dexamethasone then underwent a posterior fossa decompression, open excisional biopsy of the left cerebellar tonsil, and duraplasty. Pathology confirmed a diagnosis of medulloblastoma. The patient underwent radiation therapy to the entire neuraxis and chemotherapy with vincristine and cisplatinum.

Medulloblastoma in adults is remarkably uncommon when presenting in a multifocal fashion both in both the supra and infratentorial regions. This patient is also notable by presenting with symptoms suggestive of isolated adult Chiari malformation, but exacerbated by raised intra-cranial pressure from neoplasia.

\subsection{7}

Treatment of Trigeminal Plexiform Neurofibroma in the Very Young Patient With Ketotifen

\section{A. Price, J Wolfe, S.T. Myles, M Hamilton (Calgary, Alberta)}

Plexiform neurofibromas of the trigeminal nerve are an uncommon but well recognized occurrence in patients with neurofibromatosis 1 (NF-1). Further, the management of these lesions is typically difficult and seldom curative, especially in the very young patient where surgery and radiation therapy can bring about significant and unique problems. Traditional chemotherapy has no demonstrated efficacy in these tumors, A small experience has been reported the dermatology literature concerning the successful use of ketotifen for patients with neurofibromas. Neurofibromas have large numbers of mast cells, and these may contribute to neurofibroma growth. Ketotifen is an oral agent that blocks mast cell secretion, and is hypothesized to interfere with neurofibroma growth. This agent has few reported side effects.

We report the preliminary experience concerning treatment of 2 children with NF-I using ketotifen. The first child was age 10 months at first presentation with a rapidly growing large right trigeminal plexiform neurofibroma. She was treated with ketotifen $0.5 \mathrm{mg}$ bid for a total of 12 months starting at age 12 months. Tumor growth slowed dramatically for the first 9 months then started to increase. Surgery was performed at $21 / 2$ years. The second child presented at age $31 / 2$ years with an expanding large trigeminal plexiform neurofibroma. He was treated with ketotifen $1.0 \mathrm{mg}$ bid and has had no tumor growth for over 16 months. Neither child suffered any side effects from ketotifen.

Ketotifen is a potentially useful therapy for plexiform neurofibroma. While not definitive treatment, it is relatively low risk, can offer symptom relief (from rapidly growing tumors), and in the very young patient allow postponement of other more invasive therapeutic options, such as surgery.

\section{4-18}

The Geographic Distribution of Glioblastoma Multiforme in Nova Scotia

B. A. Williams, R. Dewar, S. Kirby, I. Mendez (Halifax, Nova Scotia)

Background: Although the overall rate of brain tumor occurrence in Nova Scotia is known, no studies are available that will provide information on the incidence of glioblastoma multiforme (GBM). Furthermore, the geographical distribution of GBM in Nova Scotia is unknown. The aim of this study was to analyze the incidence and regional distribution of GBM within the Province. The population of Nova Scotia is relatively stable with a low rate of migration and immigration. Neurosurgical services in the Province are concentrated in only one tertiarycare centre which serves the whole population.

Methods: A systematic chart review of all patients with histopathological diagnosis of GBM occurring in Nova Scotia between 1991 and 1995 was reviewed. Data were collected from the regional Cancer Centre as well as from the QElI Health Sciences Centre-the only neurosurgical centre in the Province.

Results: The overall rate of GBM in Nova Scotia during 1991-1995 was 2.14 cases per 100,000 per year with most cases occurring between ages 60 and 69 . Kings County had a two fold increase in the rate of GBM when compared to the Nova Scotian average. Pictou County had a rate which was three fold greater. The Central health region had the lowest rate followed by Western, Eastern and Northern health regions. The rate of the Cape Breton Island when compared to mainland Nova Scotia was higher, but this difference was not statistically significant.

Conclusions: Although the overall incidence of GBM in Nova Scotia is comparable to other regions of Canada, the geographical distribution of GBM varies within the different regions of the Province with some counties having significantly higher rates than others. This observation warrants further investigation into the possible causes behind these increased rates and may be important in understanding the risk factors in the development of GBM.

\section{4-19}

Therapeutic Options in Giant and Invasive Pituitary Adenomas

G. Mohr, D. Sirhan, B. Sidler, T. Kader, S. Frenkiel, G. Shenouda (Montreal, Quebec)

Rationale: Pituitary macroadenomas with giant intracranial 
extensions and/or invasive characteristics are usually growing in an infiltrating fashion and encasing vascular structures. Transsphenoidal surgery often results in partial debulking and their transcranial removal is rendered difficult due to the fibrous and hemorrhagic nature of the tumors.

Material and Methods: During the past six years we have encountered 10 cases with giant extensions of Type $D$ and $E$ of the Hardy-Vezina Classification, including 4 non-secreting adenomas, 5 prolactinomas and 1 mixed GH-PRL adenoma with acromegaly but normal growth hormone levels. Two of these patients had mostly giant intrasphenoidal extensions and were treated by transsphenoidal surgery(T.S.S.) followed by radiation (RoRx). Three patients had deliberately staged T.S.S. procedures and four patients required either primary or secondary craniotomies with only partial removal. One patient with asymptomatic invasive macroprolactinoma was treated with Bromocriptine (BRCPT) alone.

Results: In patients with prolactinomas, complete response with primary BRCPT was obtained in 1 patient. Partial responses after one or two-staged T.S.S. plus BRCPT were obtained in two cases and no response to BRCPT after partial transcranial debulking occurred in 1 patient. In non-secreting adenomas with voluminous suprasellar extensions types $D$ and $E$, partial debulking after two-staged T.S.S. in two cases resulted in effective relief of visual symptoms. Partial transcranial debulking followed by radiotherapy resulted in satisfactory stabilization in 1 patient and partial transcranial debulking followed by T.S.S. also produces stabilization in 1 case. Five out of nine patients required radiation therapy so far.

\section{4-20}

\section{The Influence Of Marimastat On The Invasive And Mitotic Activity Of Cerebral Tumors}

R.F. Del Maestro, V.R. Amberger, W. McDonald, A.G.R. Del Maestro (London, Ontario)

Background: Marimastat has been developed as a matrix metalloproteinase inhibitor with the expectation that tumor cell invasion would be inhibited by this compound. This compound is presently being assessed in a Phase III Trial in patients with glioblastoma and gliosarcoma. These experiments were carried out to assess the influence of Marimastat on the mitotic and invasive activity of a number of brain tumor cell lines, spheroids and human tumor explants in implantation culture.

Methods: C6 astrocytoma and U251 (human glioblastoma derived cell line) were assessed in monolayer culture. Invasive activity was studied using spheroid and human explant in three dimensional collagen type I matrices. Cell doubling times and invasive rates were assessed at clinically relevant concentrations. (1 $\mu \mathrm{M}-1 \mathrm{mM})$.

Results: Marimastat at $\mathrm{I}$ to $100 \mu \mathrm{M}$ concentrations did not have a statistically significant influence on cell doubling times but $1 \mathrm{mM}$ concentration significantly increased doubling times. The addition of Marimastat to the media or incorporated into the collagen type I gel resulted in similar results. Marimastat at concentrations of 10 and $100 \mu \mathrm{M}$ resulted in dose dependent decreases in cell invasion in the spheroid implantation model used. Human glioblastoma multiforme cell migration in explant culture demonstrated two distinct patterns. In one group a dose dependent inhibition of cell invasion was seen while in a second group no inhibition was observed.

Conclusions: Marimastat decreases cell invasion in the spheroid implantation assay of $\mathrm{C} 6$ and $\mathrm{U} 251$ cell lines at concentrations that do not significantly influence doubling times. The two patterns of response of human glioblastoma multiforme explants invasion were found suggesting that these tumors may not all respond to Marimastat and treatment may need to be individualized.

\section{4-21}

\section{Malignant Meningiomas Transformed from Originally} Benign Meningiomas

DS Li, RA Moumdjian, MW Bojanowski, JP Bahary (Montreal, QC)

Malignant (atypical and anaplastic) meningiomas are rare brain tumours and even rarer are those transformed from originally benign meningiomas. As far as we know, the first case of such malignant transformation was reported in 1988 by Mori $\mathrm{H}$, et al. and there were only two other single-case reports thereafter. The mechanism involved has hardly been investigated. The authors reported another two newly found such cases with a brief review of the literature.

Conclusion: Malignant meningiomas transformed from a benign origin may probably have been underestimated. Routine use of immuno-histochemical technique such as MBI-I or Ki-67 and a piece of suspected invaded surrounding brain tissue should be included whenever possible in the histological examination. This can be helpful in monitoring the malignant transformation of meningioma, as well as in predicting the biological behavior and recurrence of meningiomas.

\section{4-22}

The Role of Electrocochleography and Auditory Brainstem Response Potentials in Hearing Preservation Acoustic Neuroma Surgery

SR Browning, G Mohr, JJ Dufour, JM Rappaport, A Zeitouni, C Provencal, Y Hernandez, S Surkis, S Druker (Montreal, Quebec)

Background and Objective: Between 1995 and 1998, 70 patients with vestibular schwannomas underwent surgery at the Sir Mortimer B. Davis Jewish General Hospital in Montreal. Auditory brainstem responses (ABRs) and electrocochleography (ECoG) responses were recorded in 35 of these patients in the context of hearing preservation success.

Patients and Methods: In addition to standard audiometric testing, these patients underwent perioperative and intraoperative ABR recordings between 80 and $102 \mathrm{~dB}$ HL alternating and/or rarefaction click stimulations. The classification of ABRs is based primarily on the presence and on the latencies of Waves I, III, and V, with a special emphasis on Wave V. 
Discussion: In patients with vestibular schwannomas who demonstrate preoperative functional and audiometric hearing, a severely deteriorated ABR is suggestive of severe auditory nerve compression by the tumour. The data from this patient review as well as prior experience with intraoperative $A B R$ and ECOG monitoring is presented as are other perspectives in the literature regarding these modalities as predictors of hearing present in cases of vestibular schwannomas.

Conclusions: Despite certain limitations, ABR is one tool in determining preoperatively the likelihood of hearing preservation in patients undergoing vestibular schwannoma surgery.

\section{4-23}

Intraforaminal Lesions Presenting As Nerve Sheath Tumors: 3 Case Presentations

L. Jacques, G. Mohr (Montreal, Quebec), D. Kline, S. Albrecht (New Orleans, Louisiana)

Objective and Importance: Intradural extramedullary intraforminal lesions are most of the time diagnosed as nerve sheath tumors including meningioma and/or schwannoma. They can extend to the brachial plexus and/or intraspinally. We would like to present three cases and illustrate the importance of early surgical exploration considering the diagnosis and ultimately their treatment.

Clinical Presentation: The first patient is a 44 year old male who presented with pain and numbness in the left C5-C6 distribution. The second patient is a 69 year old female with left C6$\mathrm{C} 7$ weakness and pain. The third is a 50 year old female patient with myelopathic sign and $\mathrm{C5}, \mathrm{C} 6$ and $\mathrm{C} 7$ radiculopathy.

Intervention: Two patients underwent a brachial plexus exploration via infra and supraclavicular approaches. One of these two patients required a previous laminectomy for intraspinal resection of the tumor. One patient underwent a laminectomy and C6-C7 facetectomy for intraspinal and intraforaminal resection.

Pathological Findings: Low grade well-differentiated chondrosarcoma, hemangioblastoma and low-grade leiomyosarcoma were diagnosed in these patients.

Conclusion: These tumors are rare and often difficult to diagnose. For diagnostic purposes as well as for optimal treatment, early exploration is mandatory. Adjuvant therapy in indicated cases may be of value.

\section{SPINAL CORD}

\section{5-01}

Solid and Cell Suspension Fetal Spinal Cord Grafts as a Treatment Strategy for Spinal Cord Injury in the Transected Adult Rat

\section{Altas, M. Hong, I. Mendez (Halifax, Nova Scotia)}

Background: Although intraspinal transplants of fetal spinal cord (FSC) tissue have been shown to prevent axonal degenera- tion and support axonal regrowth following spinal cord injury in animal models, the degree of axonal regrowth is limited. In order to promote axonal regeneration, we have examined a novel technique of double grafting FSC tissue to anatomically reconstruct the damaged spinal cord in an animal model of spinal cord injury.

Methods: In eight adult female, Wistar rats with complete spinal cord transections at the T8 level, a bridge graft of solid FSC tissue (E14) was placed into the transected space. In four of these rats FSC cell suspension grafts were placed into the spinal cord rostral and caudal to the transection site. Animals with transection only served as control. At 8 weeks post transplant, animals received injections of the anterograde tracer, cholera toxin $\mathrm{B}$ (CTB), into the sensorimotor cortex. Animals were sacrificed and spinal cord sections were processed for CTB and serotonin (5-HT) immunohistochemistry to identify both descending corticospinal and serotonergic fibers.

Results: In double grafted and bridge only animals, FSC grafts survived and integration with the host spinal cord was observed. In these grafted animals 5-HT positive fibers were seen extending into the bridge graft. In double grafted animals, 5-HT fibers were also seen extending up to distances of $1.3 \mathrm{~mm}$ into the distal spinal cord. In addition, double grafted animals showed a significant reduction in corticospinal tract retraction compared to controls $(\mathrm{P}<0.05)$.

Conclusions: This study demonstrates that a combination of spinal cord bridge grafts with double cell suspension transplants may rescue transected axons and promote axonal regrowth to the distal spinal cord.

\section{5-02}

Functional Recovery and Axonal Regeneration After Transplantation of Cultured Human Teratocarcinoma Neurons (hNT) in the Hemisected Cervical Cord Rodent Model of Spinal Cord Injury

\section{B. Addas, M. Altas, M. Hong, I. Mendez (Halifax, Nova Scotia)}

Background: Previous studies have shown that regeneration of the spinal cord following injury can be achieved using grafts of fetal spinal cord, schwann cells, or peripheral nerve. Although these findings are promising, functional recovery and axonal regeneration is quite limited. Therefore, the objective of the present study was to examine the ability of grafts of cultured neurons derived from a human teratocarcinoma cell line (hNT) to restore both functional and anatomical deficits in the hemisected cervical cord model of spinal cord injury in the rat.

Methods: Female Wistar rats were trained prior to surgery and skilled forepaw function was assessed using the "staircase" and "stepping" tests. Following training and pre-op assessments, all rats underwent cervical laminectomy (C3-C4 level) to expose the cord and a hemisection was performed. Rats were randomized into three groups: 7 animals received grafts of hNT cells within, above and below the transections site (double grafts); 7 animals received grafts of cells within the transection site (bridge) and 7 animals were transected only (control). Skilled forepaw function was assessed over a 12-week period and animals were then sacrificed. Spinal cords were examined 
histologically using both cholera toxin B (CTB) labeling and serotonin (5-HT) immunohistochemistry to assess axonal regeneration.

Results: There was an apparent improvement in skilled forepaw function in animals receiving grafts of hNT cells. In double grafted animals, fibers could be seen transversing through the transection site. In contrast, in bridge only grafted animals, only fibers could be seen penetrating but not transversing the transection site. No fibers could be detected in transection only animals.

Conclusions: The findings suggest that grafts of cultured human neurons using a double grafting strategy can achieve functional recovery and restoration of axonal integrity of the transected cord.

\section{5-03}

\section{Bio-Resorbable Plates and Screws in Pediatric Neurosurgery}

\section{Hamilton, D. McPhalen (Calgary, Alberta)}

Metal plates have been used in cranial surgery in adult and pediatric patients to provide rigid fixation. There are, however, problems that can occur with retained hardware and rigid fixation in pediatric patients, including transdural migration of hardware and restriction of craniofacial growth. We report our initial experience with bio-resorbable plates and screws in pediatric neurosurgery. These plates typically undergo complete resorption 9-12 months after insertion.

Since October 1997, we have performed 11 neurosurgical procedures on 11 children utilizing bio-resorbable microplates and screws. Patients ranged in age from 6 months to 12 years at the time of operation. Surgical indications included cranial vault remodeling for craniosynostosis $(n=6)$, craniotomy for tumor $(n=3)$, craniotomy for AVF $(n=1)$, and craniotomy for trauma $(n=1)$. Follow-up ranges from 1 week to 4 months. The surgical results obtained with the plates was felt to be satisfactory in all 11 patients. No complications have occurred.

Bio-resorbable plates and screws provide an excellent alternative to metal plates in the pediatric neurosurgical patient. Long term follow-up of this patient cohort is essential to ensure efficacy and safety.

\section{5-04}

\section{Lumbar Meningocoele with Accessory Appendicular Body} Parts

P Ellis, R Kerr, J Rossitor, D Soboleski, R Smith, E Sauerbrei, G Murphy (Kingston, Ontario)

This is a case of a premature male, who presented with a meningocele defect at the mid-lumbar area with a finger-like appendage. Imaging identified spinal abnormalities including dysraphism, diplomyelia and diastematomyelia, and confirmed the presence of bony components of the digit within the defect. No other systemic or intracranial abnormalities were identified in the child, who was neurologically intact. He was re-admitted to hospital for surgery at the age of $31 / 2$ months. Pre-operative imaging confirmed that the size of the appendage, including the "digital bones" had increased, and it had developed a perfectly formed nail de novo. Imaging also indicated the bony structures within the meningocele sac were consistent with a scapula and clavicle, which appeared to have grown commensurate with the patient's age. The meningocoele was repaired and all accessory tissue removed.

Imaging and pathology on the resected structures was consistent with meningocele as well as scapula and clavicle and a well formed digit. Mature fat, muscle, peripheral nerve, astroglial tissue, epidermal and endodermal components were identified. Cytogenetics was unremarkable. Incomplete Siamese twin, mature teratoma or accessory limb as part of a meningocele defect are within the differential diagnosis, and are all rare.

\section{$5-05$}

Mucopolysaccharidoses and Spinal Cord Compression: Case Study and Review of the Literature with Implications of Bone Marrow Transplantation

\section{E Kachur, RF Del Maestro (London, Ontario)}

Background: The mucopolysaccharidoses (MPS) are a group of genetically transmitted disorders caused by deficiency of the lysosomal enzymes required for degradation of glycosaminoglycans. Hurler Syndrome (HS) is one of the seven known conditions. Lack of the enzyme results in accumulation of sulphated glycoaminoglycans in tissues. Serious clinical manifestations are seen including spinal cord compression from thickening of soft tissues and vertebral abnormalities. Neurosurgical procedures and bone marrow transplantation (BMT) have improved the quality of life in these patients.

Case Study/Results: The patient is a female with HS who underwent BMT at two years of age. She presented at eight years of age with a progressive cervical myelopathy. MRI revealed soft tissue compression of the spinal cord in the upper cervical region. A suboccipital craniectomy and $\mathrm{Cl}$ through $\mathrm{C5}$ laminectomy and decompression with duraplasty was performed. A biopsy of the compressive soft tissue and lamina was consistent with MPS. Post-operative follow up showed substantial improvement in her upper extremity functioning.

Review of the literature revealed 16 cases of MPS and spinal cord compression. Only two cases of HS were found. Eight males and eight females, average age 27.1 years (4-55), 12/16 presented with spastic tetraparesis, $2 / 16$ lower extremity involvement, 1 with arm and leg, 1 with upper limbs involved only. 10/16 had documented surgical procedures of which a decompressive laminectomy occured in $9 / 10$ cases. $8 / 10$ had good - excellent improvement, 1 had mild benefit, I died on induction. 12/16 had compresion via inflitration of soft tissues such as dura and $4 / 16$ had subluxation or gibbus deformity. BMT showed potential systemic benefits for patients with HS except in the area of musculoskeletal development.

Conclusion: MPS can induce a "metabolic myelopathy". Decompressive procedures have shown significant improvement in the quality of life. BMT may allow more patients with HS to 
survive long enough to undergo neurosurgical treatment with more frequency in the future.

\section{5-06}

\section{Initial Experience with the Codman Anterior Cervical Locking Plate}

Taro Kaibara, R. John Hurlbert (Calgary, Alberta)

Introduction: Anterior approaches for cervical spine decompression and stabilization have become a routine part of neurosurgical practice. Recently, a new titanium plating system has become available that provides major advantages over earlier systems. Initial experience with this system is presented.

Methods: From June of 1996 to July of 1997, fifty-nine patients underwent anterior cervical decompression and reconstruction supplemented with plate fixation. Anterior locking plates were applied under fluoroscopic control. Most patients were placed in a hard external orthosis for 12 weeks post-operatively $(n=50)$. Two patients were treated with halo immobilization while seven had no post-operative bracing.

Results: Post-operatively, two patients experienced C5 weakness (bilateral in one), while another suffered a right lower trunk plexus deficit. One patient experienced severe dysphagia and dysphonia requiring a temporary gastrostomy tube. A superficial wound infection occurred in one patient who later presented with lumbar osteomyelitis requiring further surgery. Six patients demonstrated post-operative hoarseness, lasting greater than 6 months in 1 . One patient died from his neoplastic process. There have been no instances of hardware failure or screw backout. After a minimum of 6 months follow-up, evidence of radiographic fusion has occurred in all 56 patients receiving bone graft.

Conclusions: Initial experience with this anterior cervical locking plate suggests it to be a valuable asset to the neurosurgeon who manages disorders of the cervical spine. Features of variable angle screw penetration, unicortical bone purchase, and superior thread size provide this product with distinct advantages compared to previous generations of cervical plates. Short-term results prove this system to be both safe and effective for the augmentation of fusion constructs in the anterior cervical spine.

\subsection{7}

The Optimal Radiological Method to Assess Spinal Canal Compromise and Cord Compression in Patients with Cervical Spinal Cord Injury: Part 1: Evidence-based Analysis

\section{S Rao, M Fehlings (Toronto, Ontario)}

The goal of this study was to identify objective, published radiological criteria to assess canal compromise and cord compression in patients with acute cervical spinal cord injury (SCI). A literature search from 1966 to 1997 was performed by MEDLINE and 37 references were included for an evidencebased analysis. Lateral radiographs were the most frequent modality described to assess spinal canal size (23 references).
T1- and T2-weighted MRIs were used to evaluate spinal cord size in 7 and 4 references, respectively. The AP diameter was the most frequent measure of cervical spinal canal size (22 references). The transverse area was the most commonly used tool to assess cervical spinal cord size ( 8 references). 26 studies correlated measurements of canal and/or cord size with neurological deficits. A small AP sagittal canal diameter was found to be associated with increased neurological deficits or symptoms in 12 studies but not in 4 references. Reliability of the radiological measurements was assessed in only 7 (19\%) studies. We conclude that there no published radiological assessments of canal compromise or cord compression in patients with acute cervical SCI which are objective, quantifiable and reliable. There is a need for such outcome parameters to objectively assess the role of decompression in acute cervical SCI.

\section{5-08}

Diffuse Idiopathic Skeletal Hyperostosis Presenting as Thoracic Myelopathy Secondary to Ossification of Spinal Ligaments

B. Sherman, T.W.J. Watson, M. Omojola (Riyadh, Saudi Arabia)

Background: Neurological complications of diffuse idiopathic skeletal hyperostosis (DISH) are uncommon with dysphagia being the most frequent neurological presentation. Spinal cord compression is rare.

Method: We describe 2 cases of DISH with spinal cord compression secondary to ossification of posterior longitudinal ligament (OPLL) and ossification of ligamentum flavum (OLF).

Results: A 56-year-old female presented with progressive myelopathy precipitated by a fall. Neurological exam showed spastic paraparetic gait with $4 / 5$ weakness of all groups in both lowers, bilateral extensor plantars, lower limb hypereflexia and spinal sensory level at T7. Radiological investigations demonstrated spinal and extraspinal findings characteristic of DISH with severe cord compression at T3-4 as a result of hypertrophic OLF and OPLL. Four level laminectomy and removal of ossified ligamentum flavum resulted in good clinical recovery with only mild residual paraparesis and eventual return to full time work as a nurse. Review of spinal radiographs over the last 3 yrs. revealed one additional case of DISH with significant cord compression from pronounced OLF.

Conclusions: DISH is a relatively common disorder in patients over 50 yrs. of age. Spinal cord compression from hypertrophic OPLL and OLF is a rare but treatable cause of myelopathy in this disorder. The diagnosis is suggested by the finding on plain spine xrays of flowing ossification of the anterior longitudinal ligament and best confirmed with multilevel MRI and CT imaging of the spine. The literature on this disorder is reviewed with emphasis on the radiological features, diagnosis and surgical management. 
5-109

\section{Primary Spondylolysis of the Axis: Case Report}

\section{E. Massicotte, I.B. Ross, M. Reed (Winnipeg, Manitoba)}

Background: The etiology of pedicular defects of the second vertebra (C2) is uncertain. The aim of this case report is to examine possible etiologies.

Method: A three month old boy came to the attention of the neurosurgical service after a lateral $x$-ray of his cervical spine was taken after an unwitnessed fall. No neurological deficits or evidence of physical trauma were noted. Imaging studies performed included: CT scan, MRI and flexion/extension x-rays.

Results: The bilateral pedicular defect of the axis seemed corticated. The dynamic studies demonstrated a significant degree of subluxation and angulation of $\mathrm{C} 2$ on $\mathrm{C} 3$. The magnetic resonance image ruled out any spinal cord pathology.

Conclusion: The lack of significant evidence of trauma on physical examination coupled with the corticated edges of the defect suggested a non-traumatic etiology.

As the location of the lesions did not correspond to lines of fusion between ossification centres, a developmental variant seemed unlikely.

A congenital etiology for this type of spondylolysis is supported by the imaging of this case and four other reported cases. ${ }^{1}$

The accurate identification of the etiology for these lesions has important repercussions on patient management and therefore deserves attention.

1. Currarino, G. (1989) Pediatr. Radiol. 19:535-538.

\section{5-10}

\section{Functional Magnetic Resonance Imaging (fMRI) of Spinal} Cord Injury

ET Kiriakopoulos, AP Crawley, MG Fehlings, CH Tator, DJ Mikulis (Toronto, Ontario)

Purpose: To demonstrate the utility of functional magnetic resonance imaging ( $\mathrm{fMRI}$ ) in mapping motor and sensory cortical activation in patients with chronic spinal cord injury.

Methods: We studied patients $(n=5)$ with previous cervical spinal cord injury and some partial preservation of either motor or sensory neurologic function. All imaging was completed on a 1.5 Tesla GE Systems MR scanner. A gradient echo pulse sequence was used with an echo time of $40 \mathrm{msec}$. A spiral trajectory through $\mathrm{K}$ space with four acquisitions through 6 slices, each $7 \mathrm{~mm}$ in thickness with no interleaving was completed. A statistical analysis of resting and task images was done using the Stimulate software program (Univ. of Minnesota). Activated pixels were mapped onto high resolution $\mathrm{Tl}$ weighted MR images.

Results: fMRI was able to consistently demonstrate activation of motor, premotor and sensory cortical regions. A difference in the number of pixels activated and the degree of ipsilateral and contralateral hemispheric activation was observed and related to the degree of preservation of neurologic function.

Conclusions: fMRI can provide insight into the changes which occur at the cortical level in patients with spinal cord injury. The patterns of activation observed on fMRI maps may assist in elucidating the plasticity of the adult brain in its execution of motor and sensory tasks following spinal cord injury.

\section{CRANIAL AND PERIPHERAL NERVE}

\section{6-01}

Analysis Of Upper And Lower Extremity Peripheral Nerve Injuries In A Multi-Trama Population

V. Prasad, J. Noble, C.A. Munro, R. Midha (North York, Ontario)

Objective: To determine the prevalence, etiology, severity and associated injuries in multi-trauma patients sustaining peripheral nerve injuries at a regional trauma centre.

Methods: Patients with injuries to the radial, median, ulnar, sciatic, femoral, peroneal, or tibial nerves were identified using a prospectively collected database and a detailed chart review was undertaken.

Results: From a population of 5777 patients treated between January 1, 1986 and November 30 1996, 162 patients were identified as having a nerve injury of interest ( $2.8 \%$ prevalence). These 162 patients sustained a total of 200 peripheral nerve injuries, 121 of which were in the upper extremity. The mean age was 34.6 and $83 \%$ of patients were male. The mean injury severity score was 23.1 . Motor vehicles accidents predominated (46\%) as the cause of injury. The most frequently injured nerve was the radial nerve and in the lower limb, the peroneal nerve was most commonly injured. Diagnosis of a peripheral nerve injury was made within 4 days of admission in $78 \%$ of the cases. Surgery was required to treat $54 \%$ of patients. Head injuries were the most common associated injury, occurring in $60 \%$ of patients.

Discussion: Data presented will aid in identification and treatment of peripheral nerve injuries.

\section{6-02}

The Calcium Chelator BAPTA-AM decreases neurite regeneration after neurite transection in an in vitro model

\section{GKT Chu, CH Tator (Toronto, Ontario)}

Background: It has been hypothesized that the rise in intracellular calcium in the neuron after neurite transection is important for regeneration (Rehder V. et al, 1992, Neurosci, Ambron R.T. and Walters E.T., 1996, Molecular Neurobiology). This hypothesis was tested by attenuation of the calcium rise in neurons after neurite transection with the calcium chelator BAPTA-AM.

Methods: Superior cervical ganglion neurons were obtained from post natal day one Sprague Dawley rats then cultured on $35 \mathrm{~mm}$ plastic culture dishes coated with collagen. The dishes were scratched with a pinrake to produce tracks along which the 
neurites extended in a linear fashion. The neurons were allowed to grow for 10 to 14 days and then their neurites were transected at least $2000 \mathrm{~mm}$ away from the cell bodies with a motor driven rubber impactor. Regeneration was assessed by counting the number of new branches per neurite at 2, 6, and 24 hours after injury. Regeneration was compared between injured neurons preloaded with BAPTA-AM and injured nonloaded neurons.

Results and Conclusions: At 2 hours after injury there was minimal regrowth of either the BAPTA-AM loaded or nonloaded neurons. However, at 6 hours, the nonloaded neurons had an average of 1.6 new branches per neurite $(n=183)$ while the BAPTA-AM loaded neurons had an average of 0.4 new branches per neurite $(n=85)$, a significant difference $(p<0.0001)$. At 24 hours, the branching of both groups became too numerous to count accurately. These results suggest that the calcium ion plays a role in the regeneration of neurites after transection and that its chelation by BAPTA-AM may delay neurite regeneration.

\section{6-03}

The Stimulus for the Intracellular Calcium Rise in the Soma of Superior Cervical Ganglion (SCG) Neurons After Neurite Transection is Extracellular

\section{GKT Chu, CH Tator (Toronto, Ontario)}

Background: After neurite transection in non-mammalian neurons, it has been shown that calcium levels may rise up to $1 \mathrm{mM}$ at the tip of the cut neurite (Ziv N.E. and Spira M.E., 1995, J Neurophys.). However, post transection calcium rise in mammalian neurons is not well characterized.

Methods: SCG neurons were obtained from post natal day one Sprague Dawley rats. The neurons were then cultured on plastic culture dishes coated with collagen. The neurons were allowed to grow for 10 to 14 days and then were loaded with the fluorescent calcium indicator Fluo-3 AM. The neurites were transected at least $2000 \mathrm{~mm}$ away from cell bodies with a motor driven rubber impactor. Pre and post injury images of calcium fluorescence were obtained with an upright laser scanning confocal microscope. The injuries were performed in normal calcium ( $\left.1.8 \mathrm{mM}\left[\mathrm{Ca}^{2+}\right] \mathrm{e}\right)$ salt solution or in zero calcium salt solution buffered with $3 \mathrm{mM}$ EGTA.

Results and Conclusions: The fractional change $(\Delta \mathrm{f} / \mathrm{fo})$ in neuronal cell body calcium fluorescence after neurite transection in the normal $\left[\mathrm{Ca}^{2+}\right] \mathrm{e}$ was found to be $0.057(\mathrm{n}=487)$ in contrast, the $\Delta f / f o$ for neurons injured in a zero $\left[\mathrm{Ca}^{2+}\right] \mathrm{e}$ was found to be $0.01(n=208)$. This difference was significant $(p<0.0001)$. These results imply that after neurite transection, there is a rise in intracellular calcium at the cell body of neurons and that extracellular calcium is required for this rise. These results do not rule out a calcium induced calcium release mechanism from internal stores that may be triggered once calcium enters the cell from the external environment.
6-04

\section{Microvascular Decompression for Trigeminal Neuralgia}

\section{AM Kaufmann (Calgary, Alberta)}

It is well established that vascular compression may cause trigeminal neuralgia (TN). However, there is considerable debate regarding the prevalence and definition of significant vascular compression upon the involved nerve.

A series of 84 MVD procedures for TN were performed by the author between July 1996 and December 1997, at two university centers. This included 62 patients with medically intractable typical TN, 11 with atypical TN, and 11 with persistent or recurrent TN after MVD. The nature of vascular compression was recorded, including assessment of contact, indentation, or distortion of the nerve by the vessel.

The superior cerebellar artery (SCA) was involved in $77 \%$ of cases typical TN and caused indentation or distortion in $50 \%$. Exclusively venous or small arterial contact with the trigeminal nerve was seen in $22 \%$ with typical TN and $55 \%$ with atypical TN.

Repeat MVD for persistent TN was performed in 4 patients first treated at other centers; all were found to have indentation or distortion of the trigeminal root entry zone by the SCA. Four patients with persistent TN after locally performed MVD were found to have no further compression at early re-exploration. However, three patients with recurrent TN after 1,3 , and 8 years were found to have new arterial indentation upon the nerve at reexploration.

No major morbidity was encountered in this series. No patients developed new hearing loss, and only one patient suffered a transient post-operative CSF leak.

Vascular compression was seen in all these patients, while indentation or distortion of the nerve was more common in typical versus atypical TN. Findings at re-exploration following failed MVD depended upon the thoroughness of the primary surgical procedure. One-year follow up results will be presented, including relationship to the $\mathrm{TN}$ symptoms, duration, and nature of MVC.

\section{6-05}

Potential Hearing Loss due to Cochlear Nerve Vascular Compression

AM Kaufmann (Calgary, Alberta) JB Wahlig, JR Balzer, TJ Lovely, PJ Jannetta (Pittsburgh, Pennsylvania)

Intra-operative loss of auditory evoked potential (AEP) waveforms is an event that strongly correlates with post-operative hearing loss. While there are several potential mechanisms of injury to the cochlear nerve, acute vascular compression has not been previously described. We report two such cases and describe the successful measures to decompress the cochlear nerve and restore hearing.

Intra-operative AEP were monitored in a consecutive series of over 300 microvascular decompressions performed in a recent twelve-month period ( $\mathrm{PJJ}, \mathrm{TJL}$, and $\mathrm{AMK}$ ). In two 
patients undergoing treatment for trigeminal neuralgia, AEP waveforms suddenly disappeared completely during closure of dura. The cerebello-pontine angle was immediately re-explored and there was no evidence of hemorrhage or cerebellar swelling. The cochlear nerve and brainstem were inspected, and prominent vascular compression was identified in both patients. The offending arteries were mobilized. Decompression resulted in successful and immediate restoration of AEP. Both patients recovered without hearing loss.

These cases illustrate that vascular compression upon the cochlear nerve may disrupt function, and is reversible with microvascular decompression. Awareness of this event and recognition of AEP changes alert the neurosurgeon to a potential reversible cause of hearing loss during posterior fossa surgery.

\section{6-06}

\section{Impact of Selective Dorsal Rhizotomies on Urodynamic Function in Children with Cerebral Palsy}

J-P Farmer, O.Vernet, A-M Houle, J-L Montes (Montreal, Quebec)

To evaluate bladder function in cerebral palsy, we performed preoperative urodynamic studies (UDS) on 27 patients (15 boys, 12 girls, mean age 5 years) undergoing selective dorsal rhizotomies for spasticity. None exhibited preoperative urinary symptoms. Only 5 of the 27 studies were considered normal. All others demonstrated low bladder volumes (total, 20 below, and 30 below bladder capacity) and high full resting pressure compared with age-matched normal controls. To date, after a mean follow up of 1 year, 8 patients have been re-evaluated postoperatively. They all demonstrate a significantly increased total, 20 below and 30 below bladder capacity. Full resting pressure tends towards lower values, without reaching statistical significance. We conclude that cerebral palsy patients have clinically silent neurogenic bladders and that rhizotomy, just as it improves tone in the lower extremities, tends to improve bladder capacity, hopefully, to the point of reducing the risk of upper urinary tract deterioration.

\section{BASIC NEUROSCIENCES}

\section{7-01}

Transient Focal Cerebral Ischemia Did Not Induce Apoptotic Neuronal Injury in Rats

\section{H. Li, P. Sun, F. Colbourne, A.M. Buchan (Calgary, Alberta)}

Introduction: Many reports from several laboratories have supported a pathway of neuronal death by an apoptotic mechanism in animal models of cerebral ischemia. z-VAD.FMK and zDEVD.FMK are peptide inhibitors of the caspase family and are neuroprotective in animal ischemic models. z-VAD.FMK and zDEVD.FMK were tested in a transient focal ischemic model.

Methods: Adult, spontaneously hypertensive rats were subjected to 90 minutes of focal cerebral ischemia by common carotid artery and right middle cerebral artery occlusion, fol- lowed by reperfusion for 22.5 hours. z-VAD.FMK (320 ng), zDEVD.FMK (320 ng), and 3\% DMSO as a control were injected intraventricularly at 60 minutes of ischemia. Rectal temperature of the rats was maintained at $37.5 \pm 0.5 \infty \mathrm{C}$ during the surgery and post-ischemic period. The volume of cortical infarction was measured.

\begin{tabular}{|c|c|c|}
\hline Results: & Group & Cortical Infarction $\left(\mathrm{mm}^{3} \pm\right.$ SD) \\
\hline & Control & $68 \pm 29$ \\
\hline & z-VAD.FMK & $97 \pm 23$ \\
\hline & z-DEVD.FMK & $75 \pm 18$ \\
\hline
\end{tabular}

Conclusion: The histological results suggest that apoptosis does not substantially contribute to infarction following focal ischemia.

\section{7-02}

No Evidence of Apoptosis Following Mild to Severe Episodes of 4-VO Ischemia in Rats

\section{F. Colbourne, H. Li, A.M. Buchan (Calgary, Alberta)}

Introduction: Forebrain ischemia leads to a delayed (24-72 hr) loss of hippocampal CAl neurons (DND). While the ultrastructural features are necrotic, recent biochemical studies implicate apoptosis. In this study, we examined, with light and electron microscopy, durations of ischemia that result in fast and slow DND. Secondly, we examined whether inhibition of caspases by z-VAD and z-DEVD would reduce DND.

Methods: Male Wistar rats (175 g) were subjected to normothermic 4-VO ischemia for $5 \mathrm{~min}$. (14 day survival) or 15 min. (7 day survival). CAl was studied by light (cell counts) and electron microscopy. In the second experiment, rats, occluded for 10 minutes ( 7 day survival), received an immediate postischemic i.c.v. injection of $320 \mathrm{ng}$ z-VAD, z-DEVD or $3 \%$ DMSO as a control.

Results: Five and $15 \mathrm{~min}$. of ischemia produced 78 and $81 \%$ CAl damage at 14 and 7 days, respectively. EM revealed extensive necrosis (e.g. mitochondrial flocculent densities, coarse tigroid chromatin) with pre-lethal injury (e.g. swollen organelles) in some neurons. No qualitative differences were noted between groups and apoptosis was not found. In the second experiment, both z-VAD (76\% dead) and z-DEVD (72\%) failed to reduce injury compared to controls (84\%).

Conclusions: No morphological or pharmacological evidence of apoptosis was evident. Thus, ischemic CAl injury is apparently not programmed.

\section{7-03}

The Effects of Post-Ischemic Behaviour Testing on CA1 Neuronal Injury in Gerbils Treated with Prolonged Hypothermia

\section{F. Colbourne, R. Auer, G. Sutherland (Calgary, Alberta)}

Background and Purpose: Five minutes of normothermic global ischemia in gerbils results in extensive hippocampal CAl loss and chronic learning impairments. While prolonged 
post-ischemic hypothermia significantly and persistently reduces most CAI loss, some neurons are only transiently salvaged. We evaluated whether functional testing would increase this slow CAI injury. Another purpose of this study was to characterize a novel behavioural test.

Methods: Gerbils were subjected to a sham operation or 5 minutes of normothermic ischemia. Of three ischemic groups, two were cooled ( $>48 \mathrm{hr}$ ) starting at $6 \mathrm{hr}$. Learning and memory were assessed by three novel small mazes, a T-maze, and an open field, except one hypothermic group who were not tested.

Results: Ischemia resulted in $81 \% \mathrm{CA} 1$ loss at 1 month, while hypothermia reduced injury to $12 \%$ and $8 \%$ in the behaviour tested and untested groups, respectively. Accordingly, protected CAI cells tolerated sustained behavioural pressure. Untreated ischemia caused hyperactivity during maze sessions on days 4 to 6 , while hypothermia treated animals were normal. The elevated maze activity, which predicted hippocampal injury, did not reflect habituation differences nor locomotor hyperactivity as this was greatest on day $\mathrm{I}$, and like normal by day 4 . Open field sessions on day 8 revealed ischemic exploratory deficits and partial protection in hypothermia treated gerbils. Ischemic working memory impairments in a T-maze were abolished by hypothermia treatment.

Conclusions: Six hour delayed cooling provided robust functional and histological protection, with CAI survival unaffected by extensive behavioural testing. These results prove that delayed hypothermia provides true neuroprotection.

\section{7-04}

\section{Development Of The Thalamostriatal Projection In The Prenatal And Postnatal Rat}

V. Srivastava, M.A. Alonso-Vanegas, C.H. Lam, A.F. Sadikot (Montreal, Quebec)

Background: Development of the thalamostriatal projection in fetal and early postnatal rats was examined with anterograde labeling using the carbocyanine dye Dil (1,1-dioctadecyl$3,3,3^{\prime}, 3^{\prime}$-tetramethylindocarbocyanine perchlorate) and biocytin(5\%). Previous studies in adult rats have demonstrated that the thalamostriatal projection originates principally from the lateral part of the parafascicular nucleus(Pf).

Methods: Dil crystals were placed in the posterior thalamus of perfused fetal rats, the brains were sectioned on a cryostat 6 months post-perfusion, and analyzed using a fluorescence microscope equipped with a rhodamine filter. Stereotactic injections of biocytin into the Pf nucleus were performed in early postnatal rats. The brains were sectioned on a freezing microtome and analyzed using a light microscope. Alternate sections were processed for calbindin-D28k to delineate the patch-matrix striatal compartments.

Results: At embryonic day 18.5, Dil-labeled axon bundles emanated from the thalamus and terminated in patches within the dorsolateral striatum. With increasing age up to embryonic day 21 , greater numbers of labeled axons were seen in the striatum. The biocytin studies demonstrated that by postnatal day 1 , the projection terminated primarily in the matrix.

Conclusion: These observations suggest that the thalamic afferents arrive in the striatum prenatally with an innervation of the matrix in the early postnatal period.

\section{7-04}

Development Of The Thalamostriatal Projection In The Prenatal And Postnatal Rat

V. Srivastava, M.A. Alonso-Vanegas, C.H. Lam, A.F. Sadikot (Montreal, Quebec)

Background: Development of the thalamostriatal projection in fetal and early postnatal rats was examined with anterograde labeling using the carbocyanine dye Dil (1,1-dioctadecyl$3,3,3^{\prime}, 3^{\prime}$-tetramethylindocarbocyanine perchlorate) and biocytin $(5 \%)$. Previous studies in adult rats have demonstrated that the thalamostriatal projection originates principally from the lateral part of the parafascicular nucleus(Pf).

Methods: Dil crystals were placed in the posterior thalamus of perfused fetal rats, the brains were sectioned on a cryostat 6 months post-perfusion, and analyzed using a fluorescence microscope equipped with a thodamine filter. Stereotactic injections of biocytin into the Pf nucleus were performed in early postnatal rats. The brains were sectioned on a freezing microtome and analyzed using a light microscope. Alternate sections were processed for calbindin-D28k to delineate the patch-matrix striatal compartments.

Results: At embryonic day 18.5, Dil-labeled axon bundles emanated from the thalamus and terminated in patches within the dorsolateral striatum. With increasing age up to embryonic day 21 , greater numbers of labeled axons were seen in the striatum. The biocytin studies demonstrated that by postnatal day 1 , the projection terminated primarily in the matrix.

Conclusion: These observations suggest that the thalamic afferents arrive in the striatum prenatally with an innervation of the matrix in the early postnatal period.

\section{7-05}

The Influence of Osteoarthritis on Articular Nerve Endings through Experimental Manipulation of the Sheep Temporomandibular Joint

RA Tedman, A Tahmasebi-Sarvestani, AN Goss (Adelaide, South Australia)

This investigation used immunohistochemistry to determine the influence of osteoarthritic changes (induced by mild condylar scarification) on the innervation of the temporomandibular joint (TMJ) of the sheep. Osteoarthritic changes were commonly seen in the anterior and lateral regions of the joint. The anterior region of the capsule had a significantly greater density of PGP 9.5- and CGRP-IR nerve fibres than other parts of the capsule in both normal and arthritic joints. Qualitative assessments suggested that the density of nerve fibres immunoreactive to antisera for PGP 9.5, CGRP and SP was less in the arthritic TMJ capsule than in the normal capsule. In addition, there seemed to be fewer nerve fibres in parts of the sheep TMJ that were most affected by the degenerative changes, for example, capsule near 
osteophytes. Nevertheless, the general linear model used to analyse the quantitative data was not able to detect any statistically significant effect of arthritis on the \% surface areas or numbers of PGP 9.5-, or CGRP-IR nerve fibres in TMJ capsule. Further work is required to determine whether parts of the joint such as synovium and bone marrow may respond differently to the experimentally-induced degenerative changes.

\section{7-06}

\section{Distractibility in Patients with Unilateral Frontal-Lobe Excisions}

\section{Koski, M. Petrides (Montreal, Quebec)}

Background: Clinical observations of distractibility in patients with damage to the frontal lobe are numerous, but experimental evidence of such a deficit is lacking. We tested whether patients with unilateral frontal-lobe excisions are easily distracted by manipulating an irrelevant stimulus dimension during performance of a manual choice reaction time task.

Methods: For a series of trials, subjects pressed one of two buttons to indicate whether the shape presented on a computer screen was a circle or a square. The colour of the shapes changed every 7,8 or 9 trials. We tested patients with unilateral excisions of the frontal or temporal lobe, as well as normal controls.

Results: We compared reaction times in the trials immediately preceding and following each colour shift, calculating a distractibility index (\% increase in reaction time). The mean distractibility index of the frontal-lobe group was significantly greater than that of the temporal-lobe group.

Conclusions: Patients with unilateral excisions in the frontal lobe may be impaired in their ability to ignore irrelevant attributes of environmental stimuli.

\section{7-07}

\section{Improving myoblast transplantation in monkeys}

D. Skuk, J. P. Tremblay (Québec, Québec)

Background: Myoblast transplantation (MT) may be a potential treatment for recessive hereditary myopathies. Since MT clinical trials showed limited results, experiments in primates are important to elucidate the human feasibility of this technique.

Methods: Macaca mulata monkeys were used for allogenic MT. Myoblasts expanded in vitro were infected with a retroviral vector encoding the $\mathrm{LacZ}$ gene. In each monkey, three muscles received $8 \times 106$ and $24 \times 106$ cells and $8 \times 106$ cells in the presence of notexin. Cells were injected at $35-50$ points using a Hamilton syringe. FK506 was given daily for immunosupression. Four weeks after MT, the transplanted sites were biopsied and the presence of $b-G a l$ muscle fibers was investigated.

Results: The number of b-Gal fibers was $1253 \pm 515$ (site grafted with $8 \times 106$ cells), $1084 \pm 278(24 \times 106)$ and $2852 \pm 1211$ (notexin). In two monkeys, b-Gal fibers in the site injected with notexin were 3782 and 3938 , including almost all the fibers in the grafted area.
Conclusions: We demonstrated that myoblasts delivered by an accurate method in the presence of notexin lead to a dramatically improvement of MT in primates. The concomitant injection of notexin was most important that the increase in the number of myoblasts. These excellent results of MT in a model that could be extrapolated to humans, suggest that MT could be effectively applied in clinical trials.

\section{7-08}

\section{Complement Deposition after Myoblast Transplantation}

D. Skuk, J. P. Tremblay (Québec, Québec)

Background: Myoblast transplantation (MT) seems to be limited by the rapid death of many transplanted cells. As complement $(C)$ could be fixed by myoblasts in vitro and as it is capable to lyse cells, we investigate its eventual role in the early death of transplanted cells.

Methods: CDI mice and Macaca mulata monkeys were used as recipients for MT. In some mice, C3 was depleted before MT using Cobra Venom Factor. Mice were sacrificed and monkeys were biopsied during the first hours after MT. Myoblast necrosis was assessed by intracellular calcium or albumin presence. C3 and $\mathrm{C} 5 \mathrm{~b}-9$ deposition was demonstrated by immunohistochemistry.

Results: In mice, C3 deposition was observed in damaged muscle fibers and in regions of necrosed myoblasts. $\mathrm{C}$ depletion did not diminish the proportion of necrosed cells. In monkeys, only a small percentage of transplanted myoblasts showed C3 or C5b-9 deposition and their proportion was not significantly different from necrosed cells.

Conclusions: Spontaneous fixation of $\mathrm{C}$ by myoblasts was not observed. $\mathrm{C}$ activation seems not implicated in damaging directly the transplanted cells and seems secondary to cellular death. Taking into account its chimiotactic functions, $\mathrm{C}$ could be implicated in the migration of neutrophils and macrophages into the clusters of transplanted cells.

\section{7-09}

\section{The Rehabilitation of Conversion Disorders}

\section{RW Teasell, AP Shapiro (London, Ontario)}

Conversion disorders often mimic neurological motor disorders and have long been thought to reflect underlying psychological conflicts. Recent behavioural theories view these as learned responses to stressful life circumstances and emphasize environmental contingencies in the maintenance of this disorder. Sixty-four patients referred to a special rehabilitation program had conversion disorders. The majority of the 64 patients were female. Thirty-two of the patients were admitted and carefully assessed on a rehabilitation unit. Paralysis was the primary presenting problem while astasia-basia was less frequently seen; in some cases both were present in the same patient. Average duration of symptoms prior to admission was 2.4 years (range 1 week to 8 years). Twenty-eight patients were initially treated utilizing a standard, often behavioural, rehabilitation approach 
but only 5 demonstrated significant or complete improvement. Seventeen of those patients who failed the behavioural rehabilitation approach were subsequently treated with a strategic behavioural "double-blind" approach. Twelve of the 17 (70.6\%) made a significant or complete recovery of physical signs of illness. An additional three patients treated with the strategicbehavioural approach alone experienced total resolution of illness. The strategic behavioural approach has proven to be initially effective in treating chronic conversion disorders. Maintenance of gains over the long-term has yet to be established.

\section{7-10}

\section{Transient Alien Hand Syndrome: A Case Report}

\section{A Nataraj, AH Rajput (Saskatoon, Saskatchewan)}

Background: Alien hand syndrome (AHS) is defined as a foreign feeling and purposeful involuntary motor activity in one's hand. It has been divided into a transient condition from an anterior corpus callosum (CC) lesion and a permanent condition from an additional mesiofrontal (MF) lesion. More recently, posterior lesions involving occipito-parietal regions, a capsulothalamic lesion with mesencephalic extension, and posterior $\mathrm{CC}$ lesions have fulfilled criteria for AHS.

Methods: We report a case of transient AHS. A video of the patient will be shown.

Results: A 47 year old right handed man presented with sudden onset of involuntary movements of his left hand and a feeling of estrangement from that hand. Involuntary grasping and picking actions with a choreic component, and dystonic posturing of the left hand were observed. MRI was normal. Symptoms resolved completely over 24 hours. Examination one month later was normal and MRI showed increased T2 signal intensity in both globus pallidi.

Conclusion: He likely had a transient ischemic attack of the right $\mathrm{MF}$ cortex and anterior $\mathrm{CC}$, as previous reports of AHS associated with ischemia have demonstrated such lesions.

\section{$7 \cdot 11$}

\section{Factitious Hemineglect and Constructional "Apraxia"}

Irfan Khan, Jeanne Ridgley, Richard Wennberg (Toronto, Ontario)

Background: It is a tenet of classical neurology that the demonstration of left hemispatial neglect and constructional "apraxia" signifies damage to the right parietal lobe.

Case report: A 45 year-old man presented with acute headache, possible seizure(s), and left-sided weakness. There was a history of near-complete recovery from a right hemisphere stroke six months previously. Examination showed a flaccid left hemiplegia/hemianesthesia with down-going plantar responses. Bedside neuropsychological testing demonstrated impaired attention, absent three-word recall, classical left hemispatial neglect on clockdrawing and an inability to copy threedimensional line drawings. CT, MRI, SPECT, CSF, EEG, evoked potentials, and hematologic/metabolic analyses were normal. Formal neuropsychological testing demonstrated an atypical pattern of bihemispheric dysfunction inconsistent with the clinical presentation. Overnight video-EEG telemetry documented the patient to move the left extremities in a normal fashion on multiple occasions.

Conclusions: To our knowledge, this is the first reported case of feigned neuropsychological dysfunction referable to the right hemisphere. The presentation suggests a diagnosis of factitious disorder or malingering, rather than conversion disorder, given the necessity of prior learning with regard to expected test results. Feigned hemispatial neglect and constructional inability must be added to the more common simulated difficulties of memory and concentration as potential confounders in neuropsychological testing.

\section{$7 \cdot 12$}

Drawing with the Non-dominant Hand: Implications for the Study of Construction Following Left Hemisphere Damage

\section{S Zacharias, A Kirk (Saskatoon, Saskatchewan)}

Background: Constructional impairment following left vs. right hemisphere damage has been extensively studied using drawing tasks. A confounding factor in these studies is that right-handed patients with left hemisphere damage (LHD) are often forced to use their non-dominant (left) hand or hemiparetic dominant hand. Qualitative differences in the drawing characteristics of left and right hand drawings by normal subjects have not previously been characterized.

Methods: Thirty right-handed, elderly subjects without a history of neurological disease were asked to draw, from memory. seven objects using the right and left hand. Half of the subjects were randomly assigned to draw with the left hand first. and half the right hand first. Right and left hand drawings were compared using a standardized scoring system utilized in several previous studies of drawing in focal and diffuse neurological disease. Each drawing was scored on eighteen criteria. Right and left hand drawing scores were then compared using the t-test for paired samples or the Wilcoxon matched-pairs test.

Results: Drawings made using the left hand were found to be significantly simpler, more tremulous and of poorer overall quality than drawings made by the same subjects using the right hand.

Conclusions: The deficits found in left versus right hand drawings of normals are similar to those found in patients with LHD, suggesting that much of the drawing impairment seen following LHD is due to an elementary motor disturbance related to use of the non-dominant hand.

\section{$7 \cdot 13$}

Evaluating The Relevance of Evidence Based Medicine in a Neurology Residency Programme

B Demaerschalk, S Wiebe (London, Ontario)

No published data exist on evidence based medicine (EBM) 
teaching programmes in Neurology. We explore teaching neurologists' (TN) attitudes towards introducing formal EBM teaching in a residency programme.

Objective: To assess TN's familiarity with EBM principles and perceived need for EBM in teaching and practice.

Methods: TNs' survey at the University of Western Ontario exploring perceived relevance of EBM in neurological training, familiarity with and practice of EBM in clinic and teaching. TNs' self-rated proficiency in EBM principles, including question formulation, evidence searching, appraisal, and application to practice. TNs and trainees rated obstacles to practice and teach EBM.

Results: Sixteen (80\%) TN and nine (100\%) trainees responded. TNs' mean ratings (scale 0 to 7 , "not at all" to "very much") were highest for EBM relevance in training (6), and lowest for familiarity and application to practice/teaching (3.7). TN's self-rated proficiency on EBM principles was 3.6, 4.6, 2.8, 3.0 respectively for formulating questions, evidence searching, literature appraisal, and application to practice. Main obstacles to EBM were insufficient knowledge for TNs, and insufficient time for trainees.

Conclusions: EBM in training/practice is highly relevant to TNs. However, proficiency in its principles is deficient. The ground is fertile for implementing EBM in neurological training.

\section{7-14}

Evidence Based Neurology Comes Of Age: Introduction To Residency Programmes

B. Demaerschalk, S. Wiebe (London, Ontario)

Background: Building on clinical skills, evidence based neurology (EBN) systematically applies the best external evidence to clinical practice. Because EBN aims at optimizing patient care, we contend that neurology residency programmes can and should incorporate formal EBN teaching.

Objective: To equip trainees with EBN principles and to foster critical analytical thinking leading to optimum patient care.

Methods: Trainees identify knowledge gaps during patient encounters, prioritizing them for discussion during biweekly or monthly teaching sessions. Core principles taught include learning to ask answerable questions, identifying sources of, searching for and critically appraising the evidence, applying best evidence to clinical problems, and self-evaluation. EBN principles are emphasized during instructors' in-service rotations. Additional sessions are held on informatics, statistics, economic evaluation and other topics of interest. Critically appraised topics are kept for future use. Instructors have formal training in neurology, clinical epidemiology and evidence based medicine.

Results: After one year, trainees adequately apply EBN principles in clinical practice and interactions with peers and clinical teachers. Implementation of EBN in residency programmes requires emphasizing clinical relevance, using interactive, nonthreatening teaching methods, teaching by example, having the necessary expert support, and maintaining continuity.

Conclusions: Comprehensive EBN teaching can be successfully implemented in neurology residency programmes.

\section{7-15}

The Effects of Butyrylcholinesterase and Acetylcholinesterase on the Growth and Survival of Dopaminergic Neurons Transplanted in the Striatum of Hemiparkinsonian Rats

K. Mukhida, M. Hong, S. Darvesh, I. Mendez (Halifax, Nova Scotia)

Background: Butyrylcholinesterase (BuChE) and acetylcholinesterase (AChE) are important enzymes in the hydrolysis of choline esters, but also have actions that may be important in neurogenesis. Previous studies have shown that $\mathrm{BuChE}$ is expressed prior to neuroblast mitosis and $\mathrm{AChE}$ is expressed post-mitosis coincidentally with axonal outgrowth. It has also been shown that $\mathrm{AChE}$ promotes dopaminergic neuron neuritic outgrowth in vitro: thus these enzymes may have a role in the neural transplantation of dopaminergic cells in Parkinson's disease (PD). The aim of this project was to study the trophic effects of BuChE and $\mathrm{AChE}$ on transplanted dopaminergic neurons in a rat model of PD.

Methods: Rat fetal ventral mesencephalon (FVM) cell suspensions were transplanted in the presence of either BuChE or $\mathrm{AChE}$ into the striatum of rats with unilateral 6-hydroxydopamine-induced lesions of the nigrostriatal pathway, the pathway involved in the pathogenesis of PD. At 8 weeks post-grafting, animals were tested for amphetamine-induced rotational behaviour, sacrificed, and their brains were examined using tyrosine hydroxylase (TH) immunohistochemistry.

Results: At 8 weeks post-grafting all animals receiving grafts of FVM cell suspensions improved in their rotational bias. Upon histological examination it was found that BuChE and AChE did not have any significant effect on the normal survival or growth of transplanted TH-positive cells when compared to animals receiving grafts of FVM cells alone. However, the cholinesterases did promote neuritic outgrowth of these transplanted TH-positive cells.

Conclusions: The findings of this study suggest that both BuChE and AChE have trophic effects on TH-positive cells in vivo in the enhancement of neurite outgrowth. This effect may have beneficial applications in neural transplantation for neurodegenerative disorders such as $\mathrm{PD}$.

\section{CEREBROVASCULAR DISEASE}

\section{8-01}

Factors Affecting the Time of Arrival to Hospital in Patients with Acute Ischemic Stroke

Abdullah BinSaeed, Huda Al-Sulaman, Ashfaq Shuaib (Edmonton, Alberta)

Background: With the understudying of the mechanisms of ischemic stroke, new therapies are being developed that show promise for clinical application. Tissue Plasminogen Activator (tPA) has recently been shown to be effective in improving clinical outcome if used within 3 hours of symptoms onset. 
Neuroprotective agents are currently being tested with a window of between 6 and 12 hours. The early arrival of patients with acute stroke to the Emergency Department is therefore essential.

Methods: In the present study we evaluated 100 patients admitted to the University of Alberta Hospital during the fiscal year 1996-97 to determine the factors that may delay hospital assessment.

Results: Of the 100 patients, 33 presented to the hospital within 6 hours and the rest within more than 6 hours from onset of symptoms. Of the patients presenting early $32 / 33(97 \%)$ lived with family, whereas only 39 of $67(58 \%)$ late presenters lived with family. $25 \%$ lived alone while $12 \%$ were in nursing homes. $58 \%$ patients presenting early were from Edmonton and came directly while only two were transferred from other hospital within the city. In patients arriving late, 26 were from the city with direct admission, 20 transferred from other hospitals within the city and 21 were transferred from outside the city. The incidence of previous ischemic heart disease was present in $36.4 \%$ patients arriving early and $15 \%$ arriving late.

Conclusions: Our data suggest that patients living with a spouse (or other family members), with a previous history of ischemic heart disease and those within the city arrive early to the hospital. Valuable time is wasted when patients are transferred from hospitals within the city.

\section{8-02}

À quelle fréquence les attaques cérébrales évaluées à l'urgence se qualifieraient-elles pour la thrombolyse? Une étude rétrospective

\section{G.M. Rémillard (Montréal, Québec)}

La thrombolyse par l'alteplase (rt PA) a été approuvée par le Food and Drug Administration (FDA) aux États-Unis le 18 juin 1996. Elle le sera vraisemblablement aussi au Canada.

Pour aider les discussions du groupe qui planifie l'utilisation de ce traitement à l'urgence, lorsqu'il sera approuvé, nous avons réquisitionné au service des archives 100 dossiers d'AVC non hémorragiques traités à l'urgence de l'Hôpital du Sacré-Coeur de Montréal.

Les patients s'étaient présentés entre janvier 1995 et mai 1996. $97 \%$ avaient été admis entre 8 h 04 et 23 h 37. Les AVC étaient d'origine thrombotique $(81 \%)$ et d'origine embolique (19\%) dont 18 associés à la fibrillation auriculaire et un à un thrombus mural. Des AVC dont l'heure du début des symptômes était clairement inscrite au dossier, les cinq qui se présentèrent assez tôt pour la thrombolyse arrivèrent en ambulance entre $8 \mathrm{~h} 25$ et $22 \mathrm{~h} 45$. La tomodensitométrie cérébrale était négative chez un seul mais cette analyse ne fut pas exécutée prioritairement dans cette série.

En conclusion, la majorité $(97 \%)$ des patients souffrant d'AVC s'étaient présentés à l'urgence, entre $8 \mathrm{~h}$ et minuit, facilitant ainsi la planification multidisciplinaire par ailleurs complexe de la thrombolyse. Les patients qui se présentèrent assez tôt (5\%) avaient tous été véhiculés en ambulance présumément via 911 .
8-03

Factors Influencing Destination after Hospitalization for Acute Stroke in Five Canadian Tertiary Care Centers

A Hakim (Ottawa, Ontario), L Lebrun (Montreal, Quebec), S Phillips (Halifax, Nova Scotia), A Shuaib (Edmonton, Alberta), P Teal (Vancouver, British Columbia), V Ramsden (Saskatoon, Saskatchewan), C Temovsky, R Dhawan, E Gudaitis, K Lawrence, P Rawn, F Shannon (Mississauga, Ontario), The OPTIMISE\%o (Optimal Management and Intervention in Stroke Emergencies) Group

Background and Objectives: Little information is currently available about the current practice patterns for stroke treatment in Canada. We have undertaken a retrospective study of the management of stroke in five tertiary care centers involved in stroke treatment in Canada and report here the factors influencing discharge destination.

Methods: A retrospective review of stroke patients admitted to five centers over the 28 month period of September I, 1994 to December 31, 1996 was conducted. Patients were identified by their ICD-9-CM code on discharge and data abstraction was undertaken by a trained Nurse-Coordinator at each site. Data were entered into Microsoft Access, and analysis was undertaken using SAS software.

Results: Data were collected on 2022 patients. Overall in-hospital mortality was $18 \%$. This was further broken down by ICD-9$\mathrm{CM}$ code into $37 \%(\mathrm{n}=372)$ for intracerebral hemorrhage (ICD $431), 14 \%(n=1071)$ for ischemic stroke (ICD 434), and 12\% ( $\mathrm{n}=578$ ) for ill-defined stroke (ICD 436, mostly ischemic). 46\% $(n=921)$ of all strokes were discharged from the tertiary care centers directly home either independently $(31 \%, \mathrm{n}=627)$ or with care $(15 \%, \mathrm{n}=294)$. Logistic regression demonstrated that patients who were married were significantly more likely to be discharged directly home, as were people who presented with predominantly sensory signs. Patients were significantly less likely to be discharged directly home if they were older, presented with motor signs or signs of neglect, had an ICD-9-CM code of 431 (intracerebral hemorrhage), or had a medical history of atrial fibrillation.

Conclusions: There are limitations to these data given the retrospective nature of the study. However, the results provide information about discharge status after stroke in five Canadian centers which may provide a useful baseline prior to the anticipated introduction of new stroke therapies in the months and years ahead.

\section{8-04}

Extended Neuroprotection Using Combination Therapy of Urokinase and Topiramate in Embolic Stroke of Rat

\section{Q. Li, Y. Yang, A. Shuaib (Edmonton, Alberta)}

Background: In this study we expected that addition of neuroprotective agent, topiramate (TPM), could enhance neural recovery of thrombolysis and reduce the risk of thrombolysisrelated intercerebral hemorrhage by decreasing the dosage of thrombolytic agent, urokinase, in cerebral ischemia. 
Methods: The embolic focal ischemia model was performed by introducing an autologous thrombus into the right-sided middle cerebral artery of experimental rats. Urokinase was infused via the right carotid artery $2 \mathrm{~h}$ after ischemic insult in two different dose settings ( $2500 \mathrm{U} / \mathrm{kg}$ group, $\mathrm{n}=6 ; 5000 \mathrm{U} / \mathrm{kg}$ group, $\mathrm{n}=8$ ) and a bolus injection of TPM $(20 \mathrm{mg} / \mathrm{kg})$ was given (i.p.) in another group $(n=8)$. Combination therapy $(n=8)$ with low-dose urokinase $(2500 \mathrm{U} / \mathrm{kg})$ and TPM $(20 \mathrm{mg} / \mathrm{kg})$ was administrated in a similar protocol. The degree of neuron damage and neurobehavior outcome were observed at $24 \mathrm{~h}$ following ischemia and compared between the groups.

Results: One animal in the group treated by high dose urokinase developed cerebral hemorrhage. The percentage of infarct volume was significantly reduced by separately-medicated urokinase and topiramate (urokinase $2500 \mathrm{u} / \mathrm{kg}, 39.1 \pm 13.0 \%$, $\mathrm{p}<0.05 ; 5000 \mathrm{u} / \mathrm{kg}, 18.4 \pm 8.5 \%, \mathrm{p}<0.001 ;$ TPM, 20.1 $111.2 \%$, $p<0.001)$ when compared to the control $(n=6,54.2 \pm 9.04)$. Addition of TPM to urokinase given at a low dose further decreased the neuron damage $(8.2 \pm 6.0, p<0.001)$, which achieved better neuroprotection than any single-drug-treated groups. Animals in combination therapy also showed better improvement in neurobehavior score. No intercerebral hemorrhage was found in low dose urokinase group and in combination therapy group.

Conclusion: Our data suggest that combination therapy of thrombolysis and neuroprotective agent may benefit stroke treatment by improving neurologic recovery and reducing the risk of cerebral hemorrhage by reducing the dosage of thrombolytic agent when it is used alone.

\section{8-05}

\section{Stroke Treatment With Ancrod Trial}

\section{DA Cameron, SE Black (Vancouver, British Colubmia)}

Ancrod, a purified fraction of venom from the Malayan pit viper (Calloselasma rhodostoma), has been shown to produce rapid and effective defibrinogenation in man. Ancrod catalyzes the hydrolysis of fibrinogen, cleaving fibrinopeptide A. The resulting hypofibrinogenemia lowers blood viscosity, thus increasing blood flow, and anticoagulates, which inhibits further thrombin formation. Ancrod also stimulates release of plasminogen activator from the endothelium with resultant reduction in plasminogen activator inhibitor and plasmin inhibitor, which may enhance local clot-specific thrombolysis.

Ancrod has been used as an anticoagulant in the management of peripheral vascular disease, deep vein thrombosis and in the presence of heparin-induced thrombocytopenia and thrombosis.

In 1993, the Stroke Treatment with Ancrod Trial (STAT) was started, which was to evaluate the safety and therapeutic efficacy of intravenously administered ancrod in patients with acute ischemic (nonhemorrhagic) cerebral infarction. The trial was completed in January of 1998 and 500 patients were recruited in total.

This was a parallel, group sequential, double-blind, randomized, placebo-controlled study of the safety and efficacy of ancrod given within 3 hours after the onset of acute, ischemic stroke.
The results of the STAT will be made known some time in 1998.

\section{8-06}

No Evidence of Neurotoxicity of t-PA Following Transient Forebrain Ischemia in Rats

\section{H. Li, P. Sun, G. Klein, A.M. Buchan (Calgary, Alberta)}

Introduction: Thrombolysis with tissue plasminogen activator (t-PA) is a potential treatment modality for acute ischemic stroke. An in vitro study of t-PA (a serine protease) has shown it to be neurotoxic following ischemia. We questioned whether it would be neurotoxic in an in vivo model, namely the 4-VO model of severe forebrain ischemia.

Methods: Adult male Wistar rats were subjected to 5 minutes of forebrain ischemia and 7 days of reperfusion. The t-PA ( 1 $\mathrm{mg} / \mathrm{kg}, 5 \mathrm{mg} / \mathrm{kg}$, and $10 \mathrm{mg} / \mathrm{kg}$ ), dissolved in $2 \mathrm{~mL}$ of sterile water, was infused intravenously at the time of reperfusion. $10 \%$ of the solution was given as a bolus and the remainder was gradually transfused over 1 hour following ischemia. Damaged CA I neurons of the dorsal hippocampus were counted and the percentage of injured cells calculated.

$\begin{array}{cc}\text { Results: } \underset{\text { Group }}{\text { \%ontrol of CAl Neuronal Injury (Mean } \pm \text { SD) }} \\ / \mathrm{kg} \mathrm{t}-\mathrm{PA} & 60 \pm 23 \\ 5 \mathrm{mg} / \mathrm{kg} \mathrm{t}-\mathrm{PA} & 66 \pm 26 \\ 10 \mathrm{mg} / \mathrm{kg} \mathrm{t}-\mathrm{PA} & 55 \pm 26\end{array}$

Conclusion: t-PA, at a therapeutic dose equivalent to that given to humans, resulted in no histological evidence of neurotoxic effects on neurons following cerebral ischemia.

\section{8-07}

\section{Dissecting Hematoma and t-PA}

\section{P. S. Kochanski, A. M. Buchan, R. N. Auer (Calgary, Alberta)}

Currently recognized complications of t-PA include intracerebral hemorrhage, but exacerbation of hemorrhage in the form of arterial dissection has not been reported.

A 24-year-old right handed male developed a left hemiplegia playing flag-football. There was no previous illness nor head trauma during the game. Glucose was $5.6 \mathrm{mM}$, pulse 108, BP 140/90. He was drowsy, with visual left neglect, facial weakness, hypertonia and hemiplegia. Eyes deviated to the right, with full ROM. There was left-sided neglect, decreased sensation and anosognosia. CT scan 75 minutes after collapse showed no definite mass effect. A total dose of $80.5 \mathrm{mg}$ of t-PA. Angiography showed suggestion of either dissection or thrombus with recanalization in the supraclinoid right internal carotid artery. Episodic bradycardia to 30 occurred at 13 hours post-ictus. Head and eyes now deviated left. Drowsiness and diaphoresis supervened. In spite of decadron, mannitol, heparin, and a right fronto-temporal decompressive craniotomy, he died 22 days after ictus. 
Autopsy showed a large, circumferential dissection of the right supraclinoid artery, extending into the anterior and middle cerebral arteries, and into the branches of the parent vessel. This case illustrates a potential hazard in the use of t-PA: extension of cerebrovascular dissecting hematoma.

\section{8-08}

Acute Trivial Trauma Associated with Intracerebral Hemorrhage Following Coronary Thrombolysis and Anticoagulation

WC Ziai, LM Metz (Calgary, Alberta)

Objective: We report two cases of intracerebral hemorrhage in patients receiving thrombolytics and/or anticoagulants following trivial acute head trauma.

Background: Thrombolysis for acute myocardial infarction may be complicated by intracerebral hemorrhage. While recent head injury is a contraindication to thrombolysis, syncope or a fall may accompany an acute thrombotic event and be considered trivial.

Results: Case one: A 50-year-old male had a myocardial infarction associated with syncope and minor head trauma. He received thrombolytic therapy followed by intravenous heparin. He developed left occipital headache 12 hours later. Cranial CT demonstrated a $1.5 \mathrm{~cm}$ hematoma in the left occipital region and a punctate hemorrhage in the right frontal region. Heparin and aspirin were immediately discontinued. Two days later the headache recurred, he became confused and the right frontal hemorrhage was now $4-5 \mathrm{~cm}$ in diameter. Case two: A 59-yearold male briefly lost consciousness and fell to the ground during a myocardial infarction. He received intravenous heparin without thrombolytics because the duration of his chest pain was unclear. The following day, he developed vertical diplopia and headache. Examination revealved a right superior oblique palsy. MRI demonstrated a small hemorrhage effacing the right side of the quadrigeminal cistern.

Conclusion: These cases suggest that when thrombolytics or anticoagulants are administered even trivial acute head trauma may be associated with increased risk of intracerebral hemorrhage.

\section{8-09}

Intracerebral Hemorrahge in the Rat: Effect of Hematoma Aspiration

M Altumbabic, MR Del Bigio, J Peeling (Winnipeg, Manitoba)

Background: The value of surgical therapy is unclear in the treatment of intracerebral hemorrhage (ICH). This study was designed to evaluate whether aspiration of hematoma in a rodent model of $\mathrm{ICH}$ could improve final neurological outcome.

Methods: ICH was induced in 37 rats by injection of bacterial collagenase into the caudate nucleus. In one group of 18 rats streptokinase injection followed by aspiration was performed 4 hours after $\mathrm{ICH}$. Behavior repeatedly evaluated until the rats were sacrificed 7 weeks after $\mathrm{ICH}$ then histological assessment was performed. Brain water was measured at 24 hours in another 14 rats.

Results: The treated rats performed significantly better $(\mathrm{p}<0.05)$ on days 1, 2 and 28 after aspiration. Skilled forelimb testing during the final 3 weeks showed a significant deficit of contralateral forelimb function in both groups, but there was no significant difference between the two groups. Neuronal loss in the striatal penumbra was significantly greater $(p<0.05)$ in untreated rats. Structural damage extended into the internal capsule and thalamus.

Conclusion: Aspiration of hematoma after experimental $\mathrm{ICH}$ in rats improved acute functional outcome and reduced neuronal loss. This is likely due to reduced space occupying effect and improved blood flow in the penumbra.

\section{8-10}

\section{Percutaneous J-Tubes In Stroke Rehab Patients}

RW Teasell, MP McRae, HM Finestone (London, Ontario)

Objective: Study was conducted examining J-tube utilization and subsequent morbidity in stroke rehabilitation patients.

Methods: Characteristics and complications of patients requiring J-tubes among 563 consecutive stroke rehabilitation admissions.

Results: $5.7 \%$ of all stroke patients admitted and $28.1 \%$ of all proven aspirators (as demonstrated by VMBS) eventually required a J-tube. $18.4 \%$ of the positive aspirators and $3.7 \%$ of all stroke rehab patients were discharged with J-tubes. J-tubes were inserted an average of 37.1 days after hospital admission. $19.1 \%$ of all brainstem stroke patients and $58.6 \%$ of aspirating brainstem stroke patients documented on VMBS required a Jtube. $11.2 \%$ of all brainstem stroke rehab patients were discharged with a J-tube. Only $2.6 \%$ of all unilateral hemispheric stroke rehab patients who aspirated required a J-tube. $34.3 \%$ of those patients who required a J-tube recovered sufficiently to allow resumption of oral feedings by time of discharge from the rehabilitation unit. J-tube non-oral feedings was not associated with prolonged institutionalization; in almost every case patients were discharged home on a home tube-feeding program.

Conclusion: J-tubes were more likely to be inserted in aspirating brainstem stroke patients. J-tubes did not cause serious complications in the aggregate.

\section{8-11}

The Use Of Percutaneous J-Tubes And Development Of Pneumonia In Stroke Rehabilitation Patients: A Comparison Of Two Hospitals

MP McRae, RW Teasell, JD Heitzner, A Bhardwaj, HM Finestone (London, Ontario)

A comparative study of J-tube insertion and its relation to the development of pneumonia was conducted by comparing two different acute/rehab stroke units with differing strategies 
towards J-tube insertions in stroke rehab patients. 563 consecutive patients admitted to the first hospital were compared to 461 consecutive patients admitted to the second hospital. There were more brainstem and hemorrhagic stroke patients admitted to the first hospital rehab unit while the population of stroke rehab patients was significantly older at the second hospital. Thirtytwo (5.7\%) stroke rehab patients had a J-tube inserted at the first hospital which was statistically significantly greater than the 14 (3.0\%) who had one inserted at the second hospital $(p<0.05)$. The difference was greatest for brainstem stroke patients $(19.1 \%$ vs. $6.1 \%$; $\mathrm{p}<0.05)$. Pneumonia developed in 12 patients $(2.1 \%)$ at the first hospital and $10(2.2 \%)$ at the second hospital (NS). The increased use of J-tubes in a consecutive group of stroke rehab patients did not appear to significantly influence the incidence of pneumonia.

\section{8-12}

\section{The Timing Of Videofluoroscopic Modified Barium Swallow (VMBS) Studies And Pneumonia In Stroke Rehab Patients: A Comparison Of Two Hospitals}

MP McRae, RW Teasell, JD Heitzner, A Bhardwaj, HM Finestone (London, Ontario)

This study was performed to determine the impact of timing of VMBS studies on the incidence of pneumonia in stroke rehabilitation patients. 563 consecutive stroke patients admitted to one hospital rehab unit in London, Ontario, Canada were compared to 461 consecutive stroke patients admitted to another hospital rehab unit in the same city. The number of initial and total VMBS studies, the timing from stroke onset to initial VMBS studies, and the incidence of pneumonia among patients in each unit was studied. The number of initial and total VMBS studies performed were $146(25.9 \%)$ and 232 respectively at the first hospital vs $55(11.9 \%)$ and 71 respectively at the second hospital $(\mathrm{p}<0.001$ ). For the first 15 days there was no difference in the number of initial VMBS studies performed $(8.53 \%$ vs. $9.54 \%, \mathrm{NS})$. The difference was accounted for by initial VMBS studies performed after 15 days $(13.68 \%$ vs. $2.17 \%, \mathrm{p}<.0001)$. The difference between both hospitals in the number of VMBS studies was not statistically significant for brainstem strokes but was statistically significant for hemispheric strokes. Pneumonia developed in 12 patients at the first hospital $(2.1 \%)$ and 10 patients at the second hospital (2.2\%) (NS). In conclusion, the more frequent use of VMBS after 15 days post stroke did not appear to influence the incidence of pneumonia among hemispheric stroke rehabilitation patients.

\section{8-13}

Does Nursing Care Really Make a Difference?

C Burgess, C Casault (Halifax, Nova Scotia)

\section{DEGENERATIVE DISEASE}

\section{9-01}

Excessive Corticomotoneuronal Drive in Amyotrophic Lateral Sclerosis

Markus Weber, Masashi Nakajima, Andrew Eisen. (Vancouver, British Columbia)

Background: To further elucidate the pathophysiology of amyotrophic lateral sclerosis (ALS) a disorder of the corticomotoneuronal system we used peristimulus time histograms (PSTHs) of the firing probability of tonically discharging motor units subjected to transcranial magnetic stimulation.

Methods: An early post-stimulus peak (primary peak) reflecting the composite excitatory post-synaptic potential (EPSP) occurs about $20 \mathrm{msec}$ after the stimulus. We compared 44 extensor digitorum communis muscle motor units from 10 normal subjects, 38 motor units from 8 patients with ALS without weakness or wasting of the examined limb (group 1), and 69 motor units from 16 patients with mild weakness and/or wasting (group 2).

Results: In $80 \%$ of normal subjects $<4$ subcomponents, reflecting stimulus-induced multiple volleys descending through the corticospinal tract, were identified within the primary peak of the PSTH. The number of subcomponents was significantly increased in both ALS groups (usually >4). In group 2 ALS the earliest subcomponent was attenuated and delayed.

Comment: The findings indicate that corticomotoneurons are hyper-excitable in ALS. This induces secondary changes in the spinal motoneuron. With progressive demise of corticomotoneurons the descending volley is, as reflected by the size of PSTH subcomponents, attenuated.

\section{9-02}

\section{Autosomal Dominant Familial ALS Pedigree with SOD1} Mutation and Prolonged Survival

KL Baerg, BJ Stewart, AH Rajput (Saskatoon, Saskatchewan), T Siddique, H Deng (Chicago, Illinois)

Background: Autosomal dominant familial amyotrophic lateral sclerosis (DFALS) accounts for 5-10\% of ALS. Fifteen to twenty percent of DFALS families have mutations of the superoxide dismutase (SOD1) gene on chromosome 2lq21.

Methods: We present clinical and neurogenetic data on a previously unreported DFALS pedigree. The SODI gene mutation was identified using single strand confirmation polymorphism analysis of polymerase chain reaction generated products.

Results: Sixteen family members were identified in four generations. Onset varied from 34 to 58 years of age. Some patients had isolated limb involvement, whereas others had both limb and bulbar involvement. Survival following diagnosis ranged from 16 to 32 years, in contrast to the accepted mean survival of 30 months. The proband had a mutation identified in exon 2 of the SOD1 gene, specifically, Gly37Arg. She is currently 69 years old and still functioning 30 years after onset of symptoms.

Conclusions: This family carries an SOD1 mutation causing DFALS. There is marked clinical heterogeneity and prolonged survival among affected individuals. 


\section{9-03}

Amyotrophic Lateral Sclerosis (ALS) Presenting as Focal Cognitive Degeneration

\section{A. Al-Asmi, H. Chertkow, S. Albrecht (Montreal, Quebec)}

Background: While cognitive changes (and frontal lobe dementia) are sometimes known to occur with ALS, only rarely do such cognitive abnormalities precede the motor symptoms or dominate the presentation.

Methods: Two case studies are presented, in which clinical presentation was that of focal cortical degeneration of the left hemisphere. Assessment with SPECT, neuropsychological testing, and eventual post-mortem results are presented.

Results: Initial clinical diagnosis was "Primary Progressive Aphasia" in one subject, and "Semantic dementia with Gerstmann's Syndrome" in the second. Four and twelve months later, motor symptoms and signs ensued. Neuropsychological evaluation and SPECT imaging provided evidence of specific left hemisphere involvement. The course was rapidly downhill in each case, dominated by spasticity in the first subject and bulbar symptoms in the second. Post-mortem exam in each case demonstrated purely ALS, with aggressive disease characterized by neuronophagia. A prediliction for the left hemisphere was noted at PM.

Conclusions: Subjects receiving a clinical diagnosis of a left hemisphere focal degenerative condition may develop ALS in the next two years, and should be followed for such.

\section{9-04}

\section{Characterization Of Dopaminergic Midbrain Neurons In A DBH:BDNF Transgenic Mouse}

M. A. Alonso-Vanegas, C. Causing, J. Fawcett, F. D. Miller, A. F. Sadikot (Montreal, Quebec)

Background: Brain derived neurotrophic factor (BDNF) has been implicated in neuronal survival, differentiation, and plasticity in the central nervous system. Dopaminergic neurons of the mouse midbrain develop according to a strict neurogenetic timetable, with birth of neurons between E10-13 and adult spatial configuration largely achieved by the early postnatal period. BDNF displays neurotrophic actions on dopaminergic neurons in cell culture.

Methods: To determine the role of BDNF in mid-brain development, we utilized a transgenic mouse (DBH:BDNF) that overexpresses BDNF in adrenergic and noradrenergic neurons as a result of fusion of the BDNF gene to the dopamine -13- hydroxylase (DBH) gene promoter.

Results: We have quantified dopaminergic neurons at four midbrain coronal levels comparing transgenic animals to wildtype control mice. Dopaminergic neurons were identified using tyrosine hydroxylase (TH) immunohistochemistry. Transgenic mice show an increase of $52 \%$ of dopaminergic cells in the midbrain, on TH and TH/Nissl stains. In confirmation, Western-blot for TH showed an increase in midbrain of transgenic animals as compared to controls.
Conclusion: We propose that DBH:BDNF transgenic mice show increased $\mathrm{TH}$ cell number in the midbrain as a result of an anterograde trophic effect of BDNF mediated by the coeruleonigral projection, resulting in rescue of dopaminergic cells from developmental cell death.

\section{9-05}

Education and Mortality in Alzheimer's Disease: Preliminary Results from the Canadian Study of Health and Aging Clinical Progression Of Dementia Study Group

CM Wolfson, H Bergman, R Bouchard, M Panisset, A Perrault, F Rouah, M Asgharian (Montreal, Quebec), I McDowell (Ottawa, Ontario), K Rockwood, S Phillips (Halifax, Nova Scotia), D Hogan, E Elby (Calgary, Alberta), S Gauthier (Verdun, Quebec), R Steenhuis (London, Ontario), S Gaerber (Vancouver, British Columbia)

Background: It has been suggested that individuals with higher education may have more advanced pathology at diagnosis of AD. This might result in an apparently higher mortality in this subgroup. Two previous studies are inconsistent.

Methods: Using data from the two phases (CSHA-I, 19911992, CSHA-2, 1996-1997) of the Canadian Study of Health and Aging (CSHA), we examined this issue. In CSHA-1, 448 probable AD subjects were identified. In CSHA-2, 427 were successfully traced and of these $328(77 \%)$ had died since CSHA-1. Missing information on education resulted in a final sample of 342 of whom 255 (74\%) had died.

Results: Using proportional hazards models, adjusted for age, gender and residence at CSHA-1 (institution or community), we found no statistically significant increased mortality in more highly educated $\mathrm{AD}$ subjects. Restricting the analysis to $171 \mathrm{AD}$ subjects with a diagnosis within three years prior to CSHA-1, the results were unchanged.

Conclusions: The relationship between education and mortality in $A D$ remains unresolved. This presentation will consider the contribution of varying methodology on the discrepant results from the three studies.

\section{9-06}

\section{Familial Cerebellar Degeneration With Early Dementia}

L.M. Bateman, A.D. Sadovnick, S. Bourque, S. Langlois, D.J. Foti (Vancouver, British Columbia)

Background: Cognitive impairment has been described with cerebellar degeneration syndromes but is rarely an early feature of sufficient extent to meet criteria for dementia. A family is described in which early learning disabilities and dementia are prominent features of a cerebellar degeneration syndrome.

Methods: Case series, pedigree and literature review.

Results: A 36-year-old woman with mild learning difficulties developed a progressive gait disorder with behavioural changes and memory loss in her early 20's. Cognitive testing indicated subcortical features with prominent executive dysfunction and memory retrieval deficits, psychomotor slowing and disinhibi- 
tion. Examination revealed cerebellar eye signs, limb incoordination and gait ataxia. Neuroimaging demonstrated marked cerebellar atrophy. Her younger sister has a similar presentation while her mother has had a progressive dementia and gait disorder since age 37 . Her daughter developed progressive learning and behavioural difficulties at age 6 and her son has mild learning difficulties. Genetic testing for SCA I, 2 and 3 was negative, with further testing in progress.

Conclusions: This family demonstrates a syndrome of cerebellar degeneration, early learning disabilities and dementia with evidence of anticipation over two generations. This may represent a phenotypic variant of an identified syndrome or a novel mutation.

\subsection{7}

\section{Apraxia In Alzheimer's Disease: Dissociating Pantomime} And Imitation

Louise Scott, Eric Roy (Waterloo, Ontario), Kira Barbour, Sandra Black (Toronto, Ontario)

Apraxia is seen in Alzheimer's Disease (AD) and has been viewed as a conceptual disorder (e.g., Ochipa, Rothi and Heilman, 1992). Benke (1993), however, suggests that there may be a form of apraxia arising from deficits in movement execution identified when patients imitate gestures. Few studies have examined whether these are clearly dissociable forms of apraxia. In this study pantomime and imitation performance of transitive gestures in a group of $30 \mathrm{AD}$ patients was compared with that in a group of matched healthy elderly. Examination of the patients' performance revealed that in pantomime, $75 \%$ of the patients were in the apraxic range ( 2 s.d. below the mean of the healthy elderly), but only $55 \%$ in imitation. Examination of individual performance revealed that while the majority of patients were impaired in both conditions, two patients were impaired only on the pantomime condition and three others only on imitation. Further, only performance on pantomime correlated with the degree of cognitive decline as measured on the MMSE. Performance on these conditions, then, is dissociable, supporting Benke's view of two forms of apraxia in AD and Roy's (1996) suggestion that apraxia may arise from disruptions at different processing stages. The implications of these findings for understanding apraxia in $\mathrm{AD}$ will be discussed.

\section{9-08}

The Value of Positron Emission Tomography in Primary Progressive Amnesia: A Case Report

Z. Arvanitakis, B. Pillon, G. Rancurel, A. Michon, A. Slachevsky, L. Naccache, Y. Samson, B. Dubois (Orsay, France)

Background: Few cases of primary progressive amnesia (PPA), the underlying pathology of which remains debated, have been reported. The present case study illustrates that PPA, although isolated, may be symptomatic of a more diffuse degenerative process.

Methods: A patient with PPA was examined serially over nine years, at the Memory Clinic of the Salpêtrière Hospital. Clinical, neuropsychological, metabolic (PET scan), and laboratory data were collected.

Results: A 66-year-old man suffered from a significant progressive memory problem since 1988. From 1991 to 1993, extensive serial neuropsychological evaluation revealed a dramatic amnesic deficit in the absence of any disturbance of global efficiency and of instrumental activities. Nevertheless, the PET scan showed bilateral temporo-parieto-frontal hypometabolism. From 1993 to 1998, neuropsychological testing demonstrated a progressive global dementing process. The patient meets the criteria for probable Alzheimer's Disease (AD).

Conclusions: Although isolated, memory deficits in PPA may be associated with a significant hypometabolism in the heteromodal cortical association areas. This observation, together with compelling evidence in the literature, suggests that PPA, even when isolated and slowly progressive, may be the first manifestation of AD. Thus, PET scanning may be useful in predicting the evolution of isolated amnesias toward a more global degenerative disorder.

\section{9-10}

\section{Dural Arteriovenous Malformation Presenting as Dementia}

\section{B.J. Stewart, K.A. Tong, A. Kirk (Sakatoon, Saskatchewan)}

Background: Dural arteriovenous malformation (AVM) is a rare cause of progressive dementia.

Methods: We present the case of a 55-year-old man with slowly progressive dementia secondary to dural AVM. CT, MRI and MRA studies are detailed.

Results: The patient presented to clinic with a several year history of incontinence, tremor and failing memory. His examination revealed dementia, rigidity and action tremor. Non-contrast CT demonstrated numerous enlarged vessels in the deep brain centrally and bilaterally. In addition, there was diffuse enlargement of scalp vessels. Subsequent MRI and MRA confirmed an extensive dural AVM involving the entire head, with arterial supply predominantly from branches of the external carotid arteries. Although neurosurgical consultation was obtained with a view to possible intervention, the patient declined any further investigation.

Conclusions: Dural AVM may give rise to a progressive dementia presumably on the basis of anoxia due to venous shunting.

\section{9-11}

\section{Progressive Generalized Dystonia in the Elderly}

A.K. Nataraj, R. Macaulay, D. George, J. Nyssen, A.H. Rajput (Saskatoon, Saskatchewan)

Background: Generalized dystonia (GD) may be idiopathic or secondary. Many causes of secondary GD have been identified but few autopsies have been reported. 
Methods: All patients were seen by one neurologist, had video documentation (included) and autopsies.

Results: Case 1: Parkinson's Disease (PD) symptoms began age 69 and were treated with Sinemet. At 78 she had GD which required Botox. She died age 79 . Autopsy revealed nigral and cortical Lewy bodies. Case 2: Age 84 she developed dysarthria and left arm stiffness which progressed to GD. Sinemet and Symmetrel were ineffective. Fasciculations and extensor plantar responses were later noted. EMG failed to reveal denervation. She died one year later. Autospy revealed motor neuron disease. Case 3: At age 60 she developed right arm apraxia which progressed to right arm dystonia, language impairment, and bilateral apraxia. Antiparkinsonian medications were ineffective. by age 64 she had GD, anarthria, and severe apraxia. She died at age 66. Autopsy revealed Pick Disease.

Conclusion: This demonstrates the spectrum of degenerative diseases which lead to GD. The dystonia was more pronounced in our PD patient than usually seen in PD. Pick Disease is well known to produce dystonia, but dystonia as a presenting feature of ALS has not been previously reported.

\section{$9-12$}

\section{Depression, Dementia and Parkinsonism: A Presentation of} Fahr's Disease

JFS Wong, M Keegan, JR Donat, K Tong (Saskatoon, Saskatchewan)

Striopallidodentate calcinosis (Fahr's disease) is a rare neurological condition defined pathologically by calcification of the small cerebral vessels located in the striatum, pallidum and dentate nucleus. These calcifications usually precede any clinical signs. Neurological manifestations include movement disorders, seizures and dementia, however; the patients may remain asymptomatic. We present a family and a single sporadic case with severe parenchymal calcifications that are consistent with a diagnosis of Fahr's disease.

The family's proband was a 59-year-old woman referred following a right hemisphere stroke. She had no cerebrovascular risk factors. Her past history included depression and parkinsonism. Laboratory studies were normal. Magnetic resonance imaging (MRI) and CT scan of the head showed extensive bilateral calcifications of the cerebellum and basal ganglia. The MRI also noted iron deposition within the basal ganglia. The patient's sister had a history of parkinsonism and a CT showed similarly severe calcification. The proband also had one son with severe depression and extensive basal ganglia calcification. The patient's parkinsonism deteriorated and she developed syncopal episodes and seizures. An EEG showed focal delta and theta activity in the left frontal temporal region. Repeat CT done three years later showed progression of the calcification.

The second case is a 68-year-old woman with a past history of major depression and a single generalized seizure. She presented with gradual memory impairment and personality change. Neurological assessment revealed mild ataxia of gait and limbs. The initial diagnosis was Alzheimer's Disease. A computed tomography (CT) scan of the head showed marked, bilateral, symmetrical calcifications within the deep white matter of the cerebrum, cerebellum, and brainstem. Metabolic studies showed normal calcium and parathyroid levels.

The presented cases demonstrate the spectrum of neurological problems associated with striopallidodentate calcinosis. Both focal and diffuse manifestations may occur. Depression was a common and prominent factor in these patients. Fahr's disease should remain in the differential of patients presenting with depression, dementia and parkinsonism.

\section{$9-13$}

\section{Poliomyelitis and Later Development of Parkinson's Disease}

A Nataraj, AH Rajput (Saskatoon, Saskatchewan)

Background: Polio (PM) is a viral disease commonly affecting anterior horn cells. Parkinson's Disease (PD) is an idiopathic degenerative disease of the substantia nigra. Some viral infections are reported to cause parkinsonism and viruses have been implicated in PD's etiology.

Methods: We report four cases of poliomyelitis with later development into PD.

Results: 1. PM age 23 caused left leg and right hand weakness. Left hand tremor began age 53. Diagnosis of PD was made at age 61 . At age 66 the tremor was confined to the left arm without medications. 2. PM occurred at age 18 without residua. Hand tremor since age 30 changed at age 50 and PD on preexisting essential tremor was diagnosed. He was Stage 2.5 at last evaluation. 3. PM occurred at age 31, affecting the right more than left. PD began at age 71 , worse on her right side. Modecate was discontinued one month before observed features of PD. 4. Childhood PD affected the left leg. Residual signs were present at age 90 when PD features predominantly left sided were noted.

Conclusion: We are reporting 4 patients with PM and later PD - both diseases that cause cell loss in specific areas of the central nervous system.

\section{$9-14$}

\section{Conjugal Parkinson's Disease}

A Nataraj, AH Rajput (Saskatoon, Saskatchewan)

Background: Parkinson's Disease (PD) is thought to be caused by an environmental insult. Investigation of conjugal Parkinson's Disease may reveal similarities in environment exposure.

Methods: Two case of CPD seen by a Saskatoon neurologist are reported.

Results: 1 Female: PD onset in right arm at age 67 and was Stage 2.5 within one year. Despite treatment she was Stage 4.0 two years later. Male: PD onset as right hand tremor, three years before his wife. He was at Stage 2.0 initially and Stage 3.0 five years later. The couple grew up in different areas. 2. Female: Age 74 at Stage 2.0 at initial consultation. Symptom onset was undetermined. She was born and raised in rural Scotland. She 
died within one year. Male: akinetic rigid PD onset at age 57. He lived in Poland until age 4 and then in Saskatoon. Levodopa was started at age 65 . He was 72 years and Stage 3.5 at last consult.

Conclusion: Using de Rijk et al.'s prevalence of $1.4 \%$ after 55 years, conjugal PD would have a $.02 \%$ chance prevalence (.014x.014). Since our patients grew up in different areas and did not know each other, etiological exposure may have occurred independently or concurrently later in life.

\section{$9-15$}

\section{Familial Progressive Supranuclear Palsy: Two Siblings}

\section{A. Hanson, M. ten Hove, A.C. Jackson (Kingston, Ontario)}

Background: Progressive supranuclear palsy (PSP) is a neurodegenerative disorder with a typical clinical picture. The etiology is unknown and the disease usually occurs sporadically.

Methods: We have neurologically assessed and investigated a sibling pair with PSP.

Results: The proband is a 71-year-old female with a one year history of loss of balance with frequent falls. She had a supranuclear gaze palsy and bradykinesia. Her 68-year-old brother became symptomatic at age 64 and demonstrated a supranuclear gaze palsy, dysarthria with pseudobulbar features, axial and limb dystonia, and bradykinesia.

Conclusions: This sibling pair has typical features of PSP and there have been a small number of previous reports of familial PSP. Although familial PSP is unusual, genetic studies of families may provide important insights into the etiology of PSP.

\section{NEUROLOGY SEIZURE DISORDERS}

\section{0-01}

\section{A Problem-based Educational Program in Epilepsy for Primary Care Physicians and Pharmacists}

\section{R.M. Sadler,J.M. Dooley, J.M. Sargeant (Halifax, Nova Scotia)}

Background: Small group, case-based learning is an educational approach used with increasing frequency in undergraduate education. In the fall of 1997 , we offered such a program to primary care physicians and pharmacists in the diagnosis and management of patients with epilepsy.

Methods: The program was designed from a needs assessment conducted early in the year. The small group sessions were held on 2 evening sessions ( 2.5 hours each) for groups restricted to 10-12 participants. A 40 question multiple choice test of epilepsy knowledge was administered before and 6-8 weeks after completion of the program. An end of course evaluation was conducted to determine the intended and reported changes in practice, perceived effectiveness of case-based interactive learning and interdisciplinary learning.

Results: Five programs were held, with 55 participants (32 physicians, 23 pharmacists). Fifty-one ( 31 physicians, 20 phar- macists) completed the knowledge pre-test and program evaluation. The mean score on the pre-test was $21.9 / 40$. The difference between physician and pharmacist scores was not significant. The results of the post-test will be available at the end of January 1998. Upon completion of the program physicians felt enabled to rely more on the seizure history, to prescribe AEDs more effectively and to diagnose and manage patients more confidently. Pharmacists felt more confident in patient counseling. The mean overall rating for effectiveness was 4.5 (scale 1-5, with $5=$ small group is much more effective than lecture). Interdisciplinary learning was valued by both groups, although significantly less by physicians.

Conclusions: Participants and teachers perceive small group interdisciplinary case-based learning as a valuable method of teaching epilepsy diagnosis and management to physicians and pharmacists.

\section{0-02}

\section{Vigabatrin and Visual Field Defects: An Update}

D. Vézina, (Laval, Québec), C. Viereck, L. Roth, C. Gorodetzky (Kansas City, Missouri), J. Veith (Bridgewater, New Jersey)

In Canada, SABRIL ${ }^{\circledR}$ (Vigabatrin, VGB) is an antiepileptic agent indicated for the adjunctive management of epilepsy which is not satisfactorily controlled by conventional therapy and as initial monotherapy for the management of infantile spasms (West syndrome). Cases of visual field defect (VFD) have been reported in Canada and in other countries, in association with the use of VGB as add-on or monotherapy. Hoechst Marion Roussel (HMR) has initiated additional efforts to further elucidate the VFDs and their possible association with epilepsy and/or other antiepileptic drugs, including VGB. An update of the results of studies (London SABRIL Neuroophthatmology Advisory Panel Meeting, HMR Drug Surveillance Database review, Prof. Harding's Study, UK General Practice Research Database Study, Pilot Epilepsy Outpatient Study, etc.) and proposed studies will be presented.

\section{0-03}

\section{Epileptic Seizures in Children with Apalic Syndrome}

V Martyniuk (Kiev, Ukraine)

In the initial period of RCPES occurrence only the first phase - generalized tonic convulsions which were mainly observed in extensor muscle groups of upper and lower extremities and lasted up to 10-20 seconds was diagnosed. Paroxysm was accompanied by spontaneous expansions of pupils, apnoea, and vegetative disorders.

The connection of the second phase of paroxysm with the majority of the sick was observed throughout $7-14$ days from the beginning of the tonic phase occurrence. Among the basic RCPES clinical features of this phase a psycho-emotional component and mimic equivalents presence should be noted.

The characteristic electric encephalography RCPES patterns 
included low in amplitude slow wave (delta - teta rhythm) EEG and the presence of atypical diffuse hot-bedded signs of fragmentary or constant paroxysm activity manifested themselves as pointed slow waves, reduced "peak slow wave" complexes with a frequent lateralisation of pathological activity into, primarily, temple and crown of the head parts (more often into a left part) of cerebral hemispheres.

The reflex conditioned polymorphous epileptic seizures described with the sick children having apalic syndrome are not listed in modern classifications of epilepsy and epileptic seizures.

\section{0-04}

\section{Marital Status and Mortality from Epilepsy}

\section{G.B.Hill (Ottawa, Ontario)}

Background: Death from epilepsy is an indicator of treatment failure. This paper examines the influence of marital status on death due to epilepsy.

Methods: Deaths from epilepsy in Canada at ages 15 and over between 1950 and 1992 were used to calculate age-standardized mortality ratios (SMR) by sex and marital status.

Results: Among women the SMR (all women = 100) was 224 for the single, 97 for the widowed, 202 for the divorced and 54 for the married. Among men the SMR was 247 for the single, 143 for the widowed, 330 for the divorced and 39 for the married.

Conclusions: The married have a much lower risk of dying from epilepsy than do the unmarried. This may be due to epileptics being less likely than others to marry or re-marry, or to marriage being a protective factor for epileptics.

\section{$10-06$}

Vagus Nerve Stimulation Therapy; Nurses' Role in a Collaborative Approach to a New Program

L Klassen, K Doerksen (Winnipeg, Manitoba)

Approximately 300,000 Canadians have epilepsy. Of those, 30 percent fail to achieve satisfactory seizure control with antiepileptic drug therapy (Vagus Nerve Stimulator Study Group, 1995). The development and availability of new therapeutic options cannot be overlooked for medically intractable patients.

Chronic vagus nerve stimulation has demonstrated a 50 percent reduction in seizure frequency in 1/3 of patients with refractory partial onset seizures (Uthman, et al, 1993). Individuals undergoing this procedure require the attention of health care professionals from both the neurological and neurosurgical programs. This unique intervention demands that the patient's device be tested intra-operative, and programming begin during the immediate post-operative phase. Assessment of tolerance and side effects to vagus nerve stimulation therapy, as well as continued evaluation of the patients seizure control are necessary to direct staged programming of the device.

This poster will demonstrate how nurses from neurology and neurosurgery clinics have been able to collaborate to ensure patients needs are met. Patient education is crucial to assisting the patient through this procedure, and key points will be identified. The implementation of coordinating the approach for programming the patient's device will be depicted. Future recommendations for long term outcome measurement will be addressed.

\section{0-07}

\section{Ketogenic Diet Clinical Pathway at British Columbia's Children's Hospital}

R Jones, S Sweeney, F Zanotto (Vancouver, British Columbia)

\section{0-08}

\section{Ketogenic Diet Experience at Camp}

\section{A Allen, C Basualdo-Hammond, R Curtis (Toronto, Ontario)}

Enabling able bodied children with intractable epilepsy on the ketogenic diet to attend camp may appear a challenge. This is a complex diet where the child's daily food intake must be weighed accurately, where any extra food may reduce ketosis and cause seizures. Ketones, and in some cases, blood sugar, need to be monitored twice daily, and small amounts of fluid given regularly. Camp is an opportunity for children on the diet to participate in non-food activities. Two children on the diet attended overnight camp for two weeks. Preparation for camp was facilitated by communication between nurses/camp, family, dietician and physician. Camp nurses were responsible for management of the diet, including weighing the food and providing regular fluid allotments. Parents provided the menus, some frozen food and their scales. All campers ate together in a main dining hall. Seizures appeared to be well controlled using the ketogenic diet plus anticonvulsant therapy. The camp experience allowed the children freedom and independence, removing the daily focus away from food. The camp was successful, the children had fun and stayed in ketosis.

\section{EPILEPSY}

\section{1-01}

\section{Functional Magnetic Resonance Imaging of Seizures}

ET Kiriakopoulos, N Gupta, H Otsubo, JT Rutka, DJ Mikulis (Toronto, Ontario)

Purpose: To utilize functional magnetic resonance imaging (fMRI) to map the cortical activation that occurs during focal motor seizures and to relate the pattern of activation to structural abnormalities.

Methods: We studied a 13 year old right handed boy with refractory partial motor seizures which began at the age of eight and progressed to epilepsia partialis continua (EPC). The underlying brain pathology was consistent with Rasmussen's 
encephalitis. Right sided partial motor seizures involving the right side of the face and tongue, twitching of the right arm and tingling of the right arm and leg. The seizures were $30-45 \mathrm{sec}-$ onds in length and recurred every two minutes. fMRI was performed on a conventional 1.5 Tesla scanner with the following sequence: TR $68 \mathrm{msec}$, TE $40 \mathrm{msec}$, slice thickness $4 \mathrm{~mm}$, FOV $30 \mathrm{~cm}$, flow compensation and one signal average. The patient was positioned supine in the standard quadrature head coil and observed by a parent, a nurse clinican and a physician. No sedation was used. Images were acquired every 7 seconds in blocks of 180 images. Mean resting and activation/seizure images were calculated by determining the mean signal intensity for each pixel location in the resting and activation/seizure images. A two tailed Student's t-test was used to compare resting and activation /seizure images.

Results: fMRI was able to demonstrate activation related to clinical seizures. Activated regions included gyri that were structurally abnormal on T2 weighted MR imaging.

Conclusions: fMRI can provide insight into the dynamic events that occur in the epileptic brain. If this technique can also be used to detect subclinical and interictal cortical activation then it could be applied more widely in the investigation of patients with intractable seizure disorders.

\section{1-02}

\section{Surgery For Temporal Lobe Epilepsy In Patients 50 Years Of Age And Older}

W. Boling, F. Andermann, F. Dubeau, A. Olivier (Montreal, Quebec)

Background: The efficacy and associated complications of epilepsy surgery is not completely known for older patients

Methods: All of the patients, 50 years of age or older, since 1981 who were treated with surgery at the Montreal Neurological Institute (MNI) for temporal lobe epilepsy were included in the study. None of the patients included had a tumor or mass in the temporal lobe.

Results: Nineteen patients were treated with either a selective amygdalohippocampectomy or cortical amygdalohippocampectomy. Postoperatively, over a mean follow-up period of four years, sixteen patients $(84 \%)$ realized an improvement, becoming either seizure-free or having better than $90 \%$ improvement in seizure frequency. One patient $(5 \%$ ) had a better than $50 \%$ but less than $90 \%$ improvement, and two patients (11\%) had a less than $50 \%$ improvement in seizure frequency. One patient $(5 \%)$ had a postoperative complication of both an upper quadrantanopsia and a scalp infection. There were no mortalities. Twelve patients $(63 \%)$ have had a reduction in antiepileptic medication. Eleven patients (58\%) have been able to return to work or to maintain an active lifestyle postoperatively. Sixteen patients $(84 \%)$ stated that they were satisfied with surgery, and they would choose surgery again. When this older cohort of patients was compared to a younger group of adult patients treated at the MNI, a similar good outcome and low complication rate was found.

Conclusion: Surgery for temporal lobe epilepsy appears to be efficacious for older patients, and it carries a small risk for postoperative complications.

\section{1-03}

Craniotomy Under Neurolept Anaesthesia - Indications and Techniques

Ramesh L. Sahjpaul, John P. Girvin (London, Ontario)

Craniotomy under neurolept anaesthesia is undergoing somewhat of a resurgence in popularity of late. More and more neurosurgeons are appreciating the advantages of operating on an awake patient.

The technique has been successfully used in over 1500 cases at this institution over the past 20 years. We reviewed our recent 4 year experience in 249 cases of which 114 were tumors, 17 vascular lesions and 118 patients' with mesial temporal sclerosis. Significant intraoperative complications directly attributable to the neurolept technique were rare. Intraoperative cortical stimulation was successful in the majority of patients. This review serves to illustrate the broad indications of neurolept anaesthesia, the ease and safety with which it can be utilized, the results of intraoperative cortical stimulation, and potential pitfalls.

\section{$11-04$}

\section{Mechanisms Governing Cell Proliferation in the Developing Forebrain: Role of NMDA Receptors}

A.F. Sadikot, A. Burhan, M.-C. Bélanger, R. Sasseville (Montreal, Quebec)

Introduction: Cell replacement strategies have been proposed for a wide variety of neurological diseases including Parkinson's disease, Huntington's disease, trauma, and epilepsy. Transplants of proliferative embryonic neuronal tissue are the most widely used method of cell transplantation. A major obstacle to current cell replacement strategies is poor survival of harvested tissues. Better knowledge of mechanisms governing neuronal proliferation is necessary to wider application of neural grafts. We hypothesized that glutamate influences cell proliferation of neural progenitors in the forebrain germinal zone by paracrine mechanisms

Methods: Using bromodeoxyuridine-based techniques, we first characterized in detail the neurogenesis of a subpopulation of GABAergic interneurons in the developing rat striatum, the parvalbumin (PV) neurons. Next, we administered the non-competitive and competitive NMDA receptor antagonists MK-801 and CGS-19755 to pregnant rats during the period of PV neuroblast proliferation, or in the post-proliferative period.

Results: We have found a marked decrease in striatal PV neuron number following NMDA receptor antagonist administration in the proliferative phase (E14-E18), but not in the postproliferative phase (E18-E22). This suggests that neuronal proliferation of subpopulations of striatal neurons is a glutamate-dependent process.

Conclusions: Besides neurobiological importance, these 
experiments suggest novel methods for improving the neuronal yield of embryonic grafts, and also have implications for mechanisms of striatal dysgenesis.

\section{1-05}

Role of the Entorhinal Cortex in the Surgery of Temporal Seizures

W Feindel (Montreal, Quebec)

Background: For many years, the amygdaloid complex and hippocampus have figured prominently in the pathophysiology and therapeutic surgery of temporal lobe epilepsy. The juxtaposed entorhinal cortex, however, has been largely neglected in relation to this variety of epilepsy, but recent evidence for rich reciprocal entorhinal-cortical connections and the strategic position of this zone as a "gateway" to the hippocampus, prompts examination of the significance of this cortical area in temporal lobe seizures.

Methods: Examples of reproduction of epileptic auras and seizure discharges from stimulation of the entorhinal cortex during operation were reviewed. Magnetic resonance imaging results were examined to identify the extent of the anatomical resection involving the entorhinal cortex in samples of the three main patterns of surgical removal now commonly practised for temporal lobe epilepsy: (a) the "standard" temporal lobectomy, with excision of the temporal cortex, amygdala and hippocampus (b) temporal lobectomy with radical removal of the amygdala and minimal excision of hippocampus and (c) amygdalohippocampectomy.

Results: (1) Some of the early examples of stimulation producing auras and seizure discharges clearly implicated the entorhinal and "uncinate cortex" (2) MRI demonstrates that a major ablation of the entorhinal cortex is a common factor in all three patters of surgical excision.

Conclusions: Evidence from stimulation of the entorhinal cortex and its major ablation in the three patterns of surgical excision now in vogue, indicates the significance of this unique cortical zone in the genesis and surgical treatment of temporal lobe seizures.

\section{1-06}

\section{The Analysis of Mitochondrial Multicomplexes Deficiency in} Parkinson's Disease

\section{U Lee, YM Yoo, YJ Kim, CJ Lee (Inchon, South Korea)}

Parkinson's disease (PD) is a neurodegenerative disease involving mainly the loss of dopaminergic neurons in substantia nigra. The cause of dopaminergic cell death is unknown. Recently, it has been suggested that PD results from mitochondrial dysfunction. In previous studies, it was found that a 5 kilobase $(\mathrm{kb})$ deletion derived from mtDNA dysfunction. Blood samples were collected from 6 positive control(PC) and 9 PD patients. Total DNA was extracted twice with phenol followed by chloroform:isoamylalcohol(24:1). To analyse of mtDNA, long and accurate polymerase chain reaction(LA PCR) was performed using mitochondrial specific primers. Deletions of large quantities were detected in several regions of mtDNA in PD patients. Analysis of the partial sequence of the mitochondrial D-loop gene using restriction fragment length polymorphism (RFLP) techniques was performed to investigate the point mutation and nucleotide sequence variation between $P C$ and $P D$ patients. Fragment variation between $P C$ and $P D$ was seen in the fragment digested by Hind III, EcoR V. Point mutation was observed in the D-loop region. In conclusion, the deletion of mtDNA occurred in complex I,III,IV and V subunits in PD patients.

\section{1-08}

Seizure Control, Emotional Adjustment and Cognitive Function After Temporal Lobe Resections that Include Neocortex

\section{IB Ross, JL Arnett (Winnipeg, Manitoba)}

Background: Recently, there has been a popularization of selective amygdalohippocampectomy for the surgical treatment of temporal lobe epilepsy (TLE). This procedure is attractive as a lesser volume of cerebral tissue is removed. To date, however, no advantage has been established. The purpose of the present study was to examine seizure control and neuropsychological test results in patients who had undergone TLE surgery that included resection of neocortical tissue.

Methods: Between 1992 and 1997 twenty TLE patients were tested pre-operatively and at two years post-op for emotional adjustment, cognitive function and seizure control. All operations included removal of a minimal amount of neocortical tissue in addition to amygdala and hippocampus.

Results: Neuropsychological testing showed improved emotional adjustment and excellent cognitive functioning, except for memory (Wechsler Memory Scale, Boston Naming Test, both $\mathrm{p}<0.05)$, after surgery. Thirteen patients $(65 \%)$ were seizure free.

Conclusions: A standard surgical approach to TLE, with minimal neocortical resection, results in good seizure control and neuropsychological function. Memory deficits are probably due to hippocampal resection and might be expected after selective amygdalohippocampectomy. Deafferentation of neocortical structures after the selective operation may result in a physiological situation similar to that after the standard operation. Further study is necessary.

\section{1-09}

Pharmacological Acceleration of Astrocyte Senescence and Memory Impairment in Rats

R.St. Jacques, G. Mohr, C.A. Chapman, J-C Lacaille, H.Schipper (Montreal, Quebec)

One characteristic of brain aging is intramitochondrial accumulation of ferrous iron in astrocytes, which can amplify oxidative stress via Fenton reactions. In primary rat astroglial 
cultures, the sulfhydryl agent, cysteamine (CSH) promotes intramitochondral iron sequestration and the formation of peroxidase-positive cytoplasmic inclusions akin to that observed in the normal aging brain. In the present study, we determined whether intracerebroventricular (ICV) infusion of CSH (1 $\mathrm{mg} /$ day; for 3 weeks plus 1 month washout) in 20-22 week old rats elicits senescence -like astroglial granulation and memory impairment. After training the animals in the three panel runway apparatus, the drug was infused into the lateral ventricle for a 3week period using a mini-osmotic pump. The animals were tested immediately thereafter for memory deficits, and the pumps were removed. After an additional one month drug washout period, the behavioral tests were repeated. The animals' brains were evaluated for endogenous peroxidase activity (ferrous iron) and GFAP immunoreactivity (astrocyte marker). In a subgroup of animals, CAl synaptic transmission was assessed using evoked field potentials in hippocampal slices. The results indicate that ICV infusion of CSH increases the number of peroxidase-positive astroglial granules in CA1 hippocampus, impairs memory processes, and reduces paired-pulse depression without significant effects on basal excitatory synaptic transmission or induction of long-term potentiation. The behavioral and electrophysiological abnormalities persist long after termination of CSH treatment suggesting that they are related to the accelerated hippocampal gliopathy and not to the neuroactive properties of the drug itself. Our model may shed light on the cellular mechanisms of brain aging and associated memory impairment and facilitate testing of cognitive enhancing and anti-dementia drugs (Supported by the Sandra Kolber Research Fund)

\section{CEREBROVASCULAR DISEASE}

\section{2-01}

\section{Image Guidance in Planning Stereotactic Radiosurgery for the Treatment of Arteriovenous Malformations}

ET Kiriakopoulos, D Scorra, K Sidhu, ML Schwartz (Toronto, Ontario)

Purpose: To evaluate the individual contribution of CT, MRI and DSA in the planning of radiosurgery for treatment of arteriovenous malformations.

Methods: We studied twenty patients $(n=20)$ who were treated at the TSRCC from November 1995 to November 1997. CT, MRI and DSA data were analyzed independently and a treatment plan to enclose the $90 \%$ isodose line was created for each imaging modality. In all cases a dose of $20 \mathrm{~Gy}$ was prescribed to the margin of the AVM nidus. CT, MRI and DSA planning reviewed by three independent investigators. A statistical analysis of cone size and stereotactic co-ordinates was done to compare the treatment plans from each imaging modality.

Results: Significant differences in cone size were observed when comparing treatment plans derived from CT vs DSA $(p=0.0068)$ and $C T$ vs MRI $(p=0.0001)$. No significant difference was seen when comparing cone size for data obtained from MRI vs DSA ( $p=0.4600)$. In $15 / 20$ cases the cone size derived from CT was at least one cone size ( $>3 \mathrm{~mm}$ ) greater than that derived from either MRI or DSA. Analysis of the stereotactic co-ordinates chosen did not demonstrate a significant difference in the spatial orientation of the target lesion when comparing $\mathrm{CT}, \mathrm{MRI}$ and DSA.

Conclusions: The principle goal of stereotactic radiosurgery is to maximize dose to the target lesion and minimize dose to normal tissues. This study provides preliminary evidence to support the stereotactic treatment planning of AVMs using data obtained from MRI and DSA images.

\section{2-02}

\section{Pseudoaneurysm From a Ruptured Arteriovenous Malformation: A Case Report}

\section{A. Suhardja, R. Moulton (Toronto, Ontario)}

We describe a case report of a 33-year-old woman with a pseudoaneurysm resulting from a ruptured arteriovenous malformation.

The patient was referred for neurosurgical consultation with a 3 month history of increasing frontal headache and mild word finding difficulty. A CT scan was initially reported as showing a glioma in the left frontal lobe. Because of suspicion that the lesion might possibly be a giant aneurysm, a cerebral angiogram was ordered. The angiogram showed a left frontal arteriovenous malformation associated with a large globular vascular structure filled with contrast. The differential diagnosis preoperatively was that of a giant aneurysm associated with the AVM, a grossly dilated cerebral vein, or a pseudoaneurysm.

The lesion was excised via a left frontal craniotomy. Our main concern in case the lesion was a pseudoaneurysm was the potential consequence of removing the tamponade effect of the skull and dura in the course of exposing the lesion. We very quickly ligated the two main feeding vessels after opening the dura and no acute expansion or rupture of the lesion occurred. The pseudoaneursym was completely excised and pathological examination confirmed the absence of any vascular tissue in the wall of the lesion.

We have encountered only one other report of a pseudoaneurysm in the brain in a search of the English language literature.

\section{2-03}

Sensitivity of Computerized Tomographic Angiography for Small Cerebral Aneurysms

Kaufmann AM, (Calgary, AB) Wehner JJ, Williams RL, Yonas H (Pittsburgh, Pennsylvania)

Computerized tomographic angiography (CTA) provides excellent anatomical detail of cerebral aneurysms relative to surrounding vascular and bony structures. We compared the sensitivity of CTA to conventional angiography in the assessment small cerebral aneurysms.

Fourteen consecutive patients with saccular aneurysms measuring less than $5 \mathrm{~mm}$ in diameter were evaluated. CTA 
examinations were completed on a G.E. High Speed Advantage Helical Scanner. Multi-planar 2-D reformatted images ( 0.3 to $0.5 \mathrm{~mm}$ ) were constructed and immediately available for review on an independent console.

Conventional angiography was first performed in nine patients. Four small unruptured aneurysms were demonstrated, and equally well seen on subsequent CTA. In five other patients presenting with subarachnoid hemorrhage, the initial angiogram was falsely negative in two or equivocal for aneurysm in three. CTA performed within 24 hours thereafter demonstrated the aneurysms measuring $1,2,3,3$ and $4 \mathrm{~mm}$. In five additional patients in whom CTA was performed first, the small cerebral aneurysms were well demonstrated and no additional aneurysms were identified on follow-up conventional angiography. All aneurysms were surgically treated, and found to be saccular.

CTA is an effective, rapid and potentially cost-effective technique that may equal or at times surpass conventional angiography in the diagnosis of even small cerebral aneurysms. CTA may also be useful in the investigation of angiographically negative subarachnoid hemorrhage.

\section{2-04}

\section{Defining Target Tissue for Acute Stroke Interventions}

AM Kaufmann (Calgary, Alberta), AD Firlik, MB Fukui, LR Wechsler, CA Jungries, H Yonas (Pittsburgh, Pennsylvania)

Clinical and CT scan assessments of acute stroke patients lack sensitivity in differentiating between areas of irreversibly injured and the potentially viable ischemic tissue that represents the target of therapeutic interventions.

Cerebral blood flow (CBF) imaging with stable Xenon CT was performed in twenty patients with acute MCA occlusion, between 90 and 360 minutes following stroke onset. The areas of $\mathrm{CBF} \leq 6, \leq 10,10-20,20-30$, and $>30 \mathrm{cc} / 100 \mathrm{~g} / \mathrm{min}$ were measured and recorded as a percentage of the hemisphere. The area of final infarction was similarly determined on the 12 available follow up CT scans.

The cerebral area with $\mathrm{CBF} \leq 20 \mathrm{cc} / 100 \mathrm{~g} / \mathrm{min}$ was significantly greater in the symptomatic hemisphere $(66 \pm 17 \%$ versus $36 \pm 12 \%, p=0.0000004)$. Severe ischemia $(\leq 6 \mathrm{cc} / 100 \mathrm{~g} / \mathrm{min})$ corresponded to the final area of infarction $(37 \pm 18 \%$ versus $40 \pm 24 \%$, Pearson's correlation coefficient $=0.866$, significant at 0.01 level). However, the acute ischemic core destined to infarction was not surrounded by a widened rim of moderate ischemia, as the area with CBF $10-20 \mathrm{cc} / 100 \mathrm{~g} / \mathrm{min}$ was similar bilaterally ( $19 \pm 4 \%$ versus $20 \pm 7 \%$, $\mathrm{p}=0.792$, not significant).

Acute quantitative measurements of $\mathrm{CBF}$ delineate the area destined to infarction, while the surrounding penumbra of moderate ischemia is relatively narrow. Treatments that improve cerebral tolerance to ischemia are therefore expected to enhance acute stroke therapy, potentially in combination with interventions that facilitate re-perfusion.
12-05

\section{Endovascular Treatment of Unruptured Aneurysms}

Geneviève Milot, Daniel Roy, Jean Raymond (Montreal, Quebec)

Objective: To assess safety, morphological results, and midterm efficacy in preventing hemorrhage of endovascular GDC treatment of unruptured subarachnoid aneurysms.

Patients and Methods: We conducted a prospective observational study between August 1992 and July 1997 of 78 patients. Patients were 27 to 76 years old (mean 52 years ). There were 59 women $(76 \%)$ and 19 men $(24 \%)$. Eighty-five unruptured subarachnoid aneurysms were treated with GDC. Most common treated lesions were ICA-ophthalmic (31.8\%), basilar (27.1\%), and MCA (14.1\%). Nine aneurysms (11\%) were giant. Unruptured lesions treated by GDC were asymptomatic in 69 patients $(89 \%)$ and presented with mass effect in nine patients $(11 \%)$. Twenty-three patients $(29.5 \%)$ had previously bled from another aneurysm.

Results: Immediate angiographic results were considered satisfactory in 72 aneurysms $(84.7 \%$ ) (complete obliteration: 44.7\%; residual neck: $40 \%$ ). There were 3 residual aneurysms (3.5\%) and 10 failures (11.8\%). Early follow-up angiograms (612 months) were obtained in $58(77 \%)$ treated aneurysms. Results remained satisfactory in $94.8 \%$ of treated lesions. Late angiographic results (>18months) were available for 43 aneurysms (57.3\%) and were satisfactory in $38(88.3 \%)$. Six patients $(10.3 \%)$ required additional treatment including 2 large and 4 giant aneurysms. Procedure related complications occurred in 9 patients $(11 \%)$. Most patients had a transient or no deficit but two had a minor permanent deficits $(2.7 \%)$ with a good outcome in all patients. There was no death. No hemorrhagic event occurred during a mean clinical follow-up of 35.5 months.

Conclusion: Endovascular treatment with GDC was a safe alternative in selected patients. No bleeding episodes occurred during a mean follow-up of 35.5 months.

\section{2-06}

\section{Building a Carotid Endarterectomy Practice - A Contemporary Experience}

\section{I.B. Ross (Winnipeg, Manitoba)}

Background: Recent efforts to capture a larger share of the carotid endarterectomy (CEA) market by neurosurgeons have met with varied success. The situation is especially difficult for younger neurosurgeons, who must demonstrate an acceptable complication rate. This study was designed to determine the complication rates and those factors that altered the author's practice during his first years in practice.

Methods: From the start of practice, in March, 1993, until December, 1997, the author prospectively recorded the results of 82 consecutive CEAs. General anesthesia and standard operative technique were used. In the last 32 cases, transcranial doppler (TCD) MCA blood velocity monitoring and selective shunting were employed. 
Results: The 30 day stroke/death rate was $4.9 \%(n=4)$. There was one facial nerve injury (mandibular branch) and 6 (7.3\%) post-operative hematomas requiring evacuation. All wound hematomas occurred in patients taking ticlopidine. Aggressive perioperative control of blood pressure, problems with which were felt to contribute to two major strokes, coupled with TCDguided selective shunting, resulted in a recent string $(n=34)$ of stroke-free surgeries. Stopping ticlopidine 2 weeks pre-op prevented further hematomas.

Conclusions: Good outcomes from CEA can be obtained during the first few years in practice. Corrective measures, after analysis of complications, may improve outcomes.

\subsection{7}

Is there a Correlation Between Clinical Modes of Presentation and Radiological Haemodynamics of Cerebral AVMs? Results of a Prospective, Blinded Analysis in 31 Patients

Norris JS, Valiante T, Wallace MC, Willinsky RA, Montanera WJ, terBrugge KG, Tymianski M (Toronto, Ontario)

Introduction: Whether a cerebral AVM presents with haemorrhage, seizures or other neurological phenomena may depend upon the haemodynamics within the vessels supplying and draining it. We sought to investigate this with a prospective, blinded analysis.

Methods: Cerebral AVMs in 31 consecutive patients were imaged angiographically, using rapid sequence acquisition (3.8 images/sec) during contrast injections. From these images were derived contrast dilution curves from feeding arteries and draining veins. The times to reach $20,50,80$ and 100 per cent of either side of the maximum contrast density were determined for each vessel. We then determined whether these parameters, the arterio-venous transit time or AVM size were related to patients' presentation with haemorrhage (11 patients), seizure (II patients) or other clinical symptoms (9 patients).

Results: Significant findings were confined to analyses of arterial phase. The venous parameters, AVM size and contrast transit times through the nidus did not correlate significantly with clinical presentation. Arterial filling with contrast was significantly slower in those patients presenting with haemorrhage (mean $50,80,100 \%$ of time to peak $=1.19+/-0.13,1.97+/$ $0.18,3.04+/-0.35 \mathrm{sec}$ ) compared with seizure patients (mean $50,80,100 \%$ of time to peak $=0.80+/-0.12,1.32+/-0.18,1.95$ $+/-0.29 \mathrm{sec}$ ), ANOVA, $\mathrm{p}<0.05$, post hoc t-tests $\mathrm{p}<0.05$ for each parameter.

Conclusions: The rate of increase of contrast concentration in the arterial feeders of an AVM correlates significantly with the mode of presentation. A more rapid increase is associated with those presenting with seizures and slower in those with haemorrhage. Although not statistically significant, this trend is maintained throughout the rest of the arterial and venous phases. Further recruitment continues so that we may validate our findings.
12-08

Anterior Communicating Artery Aneurysms: Classification And Technical Considerations

Lacroix M., Bojanowski M.W., McLelland H. (Montréal, Québec)

Background and purpose: Anterior communicating artery (Acom) aneurysms are the most frequent of the anterior cerebral circulation. Even though tremendous progress has been made in their surgical management, secondary morbidity and mortality remain a preoccupying issue. Aneurysmal chararcteristics such as orientation, size and anatomical individual variations are linked to the clipping procedure difficulty. Also, secondary ischemic complications due to the surgical procedure itself correlate with a worst clinical outcome. During recent years, we have developed a surgical strategy for these aneurysms according to their relation to the $\mathrm{A} 2$ segments. We report this strategy and our results.

Material and Methods: A retrospective analysis of 50 consecutive cases of Acom aneurysm which were surgically treated in our institution, in the period between 1994 and 1996. There were 24 men and 26 women, with a mean age of 52 years. Of these, 43 were ruptured aneurysms and 7 incidental ones. The former were classified, following the Hunt and Hess $(\mathrm{HH})$ classification of subarachnoid hemorrage and were distributed as follows: 2 Grade I, 8 Grade II, 14 Grade III, 8 Grade IV and 2 Grade V. They were then redistributed in three groups defining their angiographical presentation. Type I are the aneurysms anterior to the A2 segment, type II are in the same plane as the A2 segment and type III are posterior to the A2 segment. The surgical approach was through a pteryonal procedure and its variants, according to each type of aneurysm. The Glasgow Outcome Scale was used for comparison ( a favorable outcome being a score of 1 or 2).

Results: In the group with good clinical grades (HH Grade I and II), there were 12 type I aneurysms, favorable outcome (FO): 12 (100\%) and 7 type III aneurysms, FO: 7 (100\%). In the group with intermediate clinical grades (HH Grade III), there were 7 type I, FO: 4 (63\%), 4 type II, FO: $2(50 \%)$ and 3 type III, FO: 3 (100\%). In the group with poor clinical grades ( $\mathrm{HH}$ Grade IV and V), there were 4 type I, FO: $2(50 \%), 1$ type II, FO: 0 and 5 type III, FO: $2(40 \%)$. The outcome was favorable for all the incidental aneurysms (7) independent of their angiographical presentation.

Conclusions: Acom aneurysms have to be treated surgically as a multivariant entity, each of them necessitating a specific surgical approach tailored to their relation to the A2 segment. By doing so, their prognosis depends primarily on their clinical grade instead of their morphological and anatomical characteristics.

\section{2-09}

\section{Calgary Carotid Endarterectomy Audit}

E Janes, W Ghali, H Karbalai, T Feasby, AM Buchan (Calgary, Alberta)

Purpose: Carotid endarterectomies by regional neurosurgeons 
were audited retrospectively, for the two years prior to the NASCET result release, assessing their appropriateness and complication rate.

Methods: Reviewing the charts, radiological and pathological reports, data on 112 patients (mean age $66.7 \pm 8.5$ (S.D.), $76 \mathrm{M} / 36 \mathrm{~F}$ ) were compiled regarding the post-operative complication rates, and the appropriateness of surgery as defined by the presence of $70-99 \%$ ipsilateral symptomatic stenosis, confirmed by conventional angiography.

Results: The post-operative mortality rate was $1 / 112(0.9 \%)$, the post-operative stroke rate was $8 / 112$ (7\%) (2 minor, 5 moderate, 1 major). 16 operations (14\%) were deemed inappropriate or uncertain, 8 because of asymptomatic stenoses, 2 because the vessel was occluded at operation, and the remaining 6 because the degree of stenosis was $<70 \%$ by NASCET criteria. An interesting result was that $3 / 16(19 \%)$ of the inappropriate cases resulted in stroke, while only $5 / 96(5 \%)$ of the appropriate cases had this outcome (O.R. $=4.2$, due to the small sample size, it did not reach significance: $\mathrm{p}=0.07$ ).

Discussion: It is imperative to maintain appropriateness in performing carotid endarterectomies because inappropriate surgery puts patients who will not benefit at risk, and might result in a higher complication rate. The audit will be continued prospectively, and in greater detail, subsequent to the release of the $30-70 \%$ stenosis NASCET results.

\section{2-10}

\section{Intra-Arterial Thrombolysis of Proximal MCA Occlusion}

AM Kaufmann (Calgary, Alberta), AD Firlik, MB Fukui, LR Wechsler, CA Jungries, H Yonas (Pittsburgh, Pennsylvania)

Leptomeningeal collaterals may provide adequate cortical perfusion during acute MCA thrombo-embolic occlusion, although associated obstruction of the lenticulostriate arteries may result in severe ischemia and irreversible injury. The safety of thrombolysis in this setting has not been established.

Eleven patients with acute proximal MCA thrombo-embolic occlusion underwent intra-arterial thrombolysis (IATL) using urokinase, within 360 minutes of stroke onset. Quantitative cerebral blood flow (CBF) measurements were made prior to treatment. The extent of pre-treatment ischemia was measured and compared to the final infarction on follow-up CT scans.

A lesser extent of severe ischemia was associated with successfully IATL (i.e. CBF $\leq 6 \mathrm{cc} / 100 \mathrm{~g} / \mathrm{min}$ in $22 \pm 9 \%$ versus $48 \pm 10 \% ; \mathrm{p}<0.05)$. Independent of IATL results, the area of severe ischemia corresponded to the extent of final infarction $(37 \pm 18 \%$ versus $40 \pm 24 \%$; Pearson's correlation coefficient $=0.866$, significant at 0.01 level). However, symptomatic deep intracerebral hemorrhage occurred in 3 patients, and was not related to the pretreatment extent of severe ischemia $(37 \pm 12 \%$ versus $44 \pm 15 \%$ of hemisphere; $p=0.31$, not significant).

Acute CBF measurements may predict IATL success as well as the ultimate extent of cerebral infarction. However, with proximal MCA thrombo-embolic occlusions there is an independent risk of deep hemorrhage, likely due to direct delivery of thrombolytic agents into the irreversibly injured perforator territory.
12-11

Rational Treatment of Vasospasm Following Aneurysmal Subarachnoid Hemorrhage

AM Kaufmann (Calgary, Alberta) H Yonas, AD Firlik, BL Clyde, KS Firlik, CA Jungreis, DK Resnick, M Rutigliano, EM Nemoto (Pittsburgh, Pennsylvania)

Vasospasm (VSP) remains a leading cause of morbidity and mortality following aneurysmal subarachnoid hemorrhage (aSAH). We have recently reported several studies of quantitative cerebral blood flow (CBF) measurements in patients with suspected VSP. These results are reviewed, and their significance to rational patient management discussed.

Among 95 patients with aSAH, we found that 30 developed delayed neurological deterioration and increase of transcranial Doppler (TCD) blood flow velocities. All underwent stable Xenon computerized tomography $(\mathrm{XeCT})$. Nineteen had no cerebral ischemia demonstrated, and none developed subsequent new infarcts. Conversely, 10 of 11 with $\mathrm{XeCT}$ defined ischemia had angiographically defined VSP.

Another study of 50 patients demonstrated no statistical relationship between changes in TCD blood flow measurements and $\mathrm{XeCT}$ defined cerebral ischemia or neurological deficits.

When cerebral ischemia was demonstrated in symptomatic patients, vasopressor agents usually resulted in improved CBF, although occasional paradoxical responses were observed. Intraarterial papaverine resulted in reversal of arterial narrowing in $78 \%$ of cases, while CBF augmentation was demonstrated in only $46 \%$ and major clinical improvements in only $26 \%$. Cerebral angioplasty, however, was successful in $93 \%$ with improved neurological function and significant reduction in the extent of critical cerebral ischemia.

Quantitative CBF measurements are a valuable tool in the accurate detection of symptomatic VSP, and assessment of therapeutic interventions.

\section{2-12}

\section{A Simple Technique for Intra-Operative Cerebral Angiography}

AM Kaufmann, JA Silvaggio (Calgary, Alberta)

Intra-operative angiography during cerebral aneurysm surgery may confirm complete cerebral aneurysm obliteration and full patency of the associated vessels. We describe a retrograde injection technique that has potential advantages over the traditional percutaneous trans-femoral approach.

During the initial exposure for craniotomy, the superficial temporal artery (STA) is cannulated with a 20 -gauge catheter and connected to an arterial line flush system. Digital subtraction angiography is performed with C-arm fluoroscopy during retrograde hand injection of 7-10cc of contrast. Optimal C-arm position is established during pre-clip imaging. After aneurysm clipping, repeat angiography can then be completed within 5-10 minutes.

Seven patients recently underwent intra-operative angiography 
by retrograde STA injection, during craniotomies for treatment of anterior circulation cerebral aneurysms. The technique was particularly useful in confirming patency of fetal posterior cerebral arteries after clipping of 2 associated aneurysms. In one patient with closely related posterior communicating and anterior choroidal artery aneurysms, repositioning of one clip was directed by the intra-operative STA angiogram. Furthermore, the rapid means to confirm post-clipping arterial patency facilitated optimal clip placement.

Intra-operative retrograde STA angiography is a safe, effective, and cost-effective means to rapidly confirm adequacy of clip placement during cerebral aneurysm surgery.

\section{$12-13$}

\section{Outcome Following Emergent Surgical Treatment of Aneurysmal Intracerebral Hemorrhage}

AM Kaufmann, CA Hall, (Calgary, Alberta), AD Firlik (Pittsburgh, Pennsylvania)

The recovery potential and management guidelines for individuals presenting in poor clinical condition due to an aneurysmal intracerebral hemorrhage (AnICH) are not well established.

Seventy-three ruptured cerebral aneurysms were surgically treated between July 1994 and December 1997 (AMK). We assessed the outcome of the six patients presenting with poor clinical grade (Hunt \& Hess 4-5) and large lobar AnICH, who underwent emergent surgical treatment. Glasgow Coma scores were $4 \mathrm{t}$, 4t, 5t, 5t, 6t, \& 9t, and three had fixed-dilated pupil(s). The AnICH measured 6 to $8 \mathrm{~cm}$ and resulted in 5 to $15 \mathrm{~mm}$ of midline shift. Surgical intervention was initiated within 180 minutes of ictus, with selection based upon relatively young age (30-59 years), early presentation, and AnICH outside of eloquent cerebral tissue.

Aneurysm clip obliteration was achieved in all cases with no secondary infarcts. All six patients survived and were transferred to rehabilitation centers after 19 to 30 days. Four patients returned to independent home living with Glasgow Outcome Scores of 2 and Barthel Index (BI) scores of 100. One patient remained institutionalized while the sixth was still in rehabilitation at 3 months, with a BI of 95 .

A good recovery potential exists in carefully selected poor grade patients with AnICH. Therefore, emergent treatment including hematoma evacuation and aneurysm clip obliteration should be considered, even with advanced signs of brainstem compression.

\section{2-14}

Sentinel Headache and Ultra-Early Rebleeding Influence Outcome of Aneurysmal Subarachnoid Hemorrhage

AM Kaufmann (Calgary, Alberta)

Accurate diagnosis of aneurysmal subarachnoid hemorrhage (aSAH) may reduce the associated high rate of morbidity and mortality. The influence of sentinel headache and early rebleeding upon presenting grade and outcome is presented.
A series of 71 ruptured cerebral aneurysms surgically treated between September 1994 and December 1996 (AMK) was reviewed. Twenty-three patients (32\%) had experienced a sentinel headache, and 9 had sought medical attention (11\%). Thirteen of these patients ultimately presented with Hunt \& Hess grade 3-5 aSAH (42\%).

Ultra-early rebleeding that occurred within six hours of presentation to hospital (but prior to transfer to the tertiary care center) occurred in 9 patients (12\%). All deteriorated clinically to Hunt \& Hess grade $3(n=4)$ or grade $4(n=5)$.

A good or excellent outcome was seen in 50 of the total 71 patients (70\%), including 33 of 34 who presented with Hunt \& Hess grade 1-2 aSAH (98\%). History of sentinel headache or ultra-early rebleeding was associated with a lesser chance of good/excellent outcome, seen in 13 of $23(42 \%)$ and 5 of 9 $(44 \%)$, respectively.

A favorable outcome is expected in most patients treated with a good grade aSAH. Improved recognition of sentinel headache remains a vital aspect of aSAH management, as failure to do so is associated with high risk of severe rebleeding and poor outcome. Furthermore, ultra-early rebleeding during hospitalization is a significant target of acute therapeutic interventions that may include aggressive control of hypertension.

\section{2-15}

Intra-operative Angiography and Hypothermic CardioCirculatory Arrest in Giant and Complex Aneurysms

G.Mohr, D. Tampieri, F. Salevsky, P. Goldenberg (Montreal, Quebec)

During the past year, two patients with particularly challenging lesions were treated successfully with minimal or acceptable morbidity using hyperthermic cardio-circulatory arrest and intra-operative angiography (HCCIA and IOIA).

The first patient, a 42 year old lady, previously treated elsewhere with whole brain radiation for suspected multiple intracranial melanoma - metastases was found to have mirror MCA-aneurysms, consisting of left-sided giant MCA-bifurcation aneurysm and a small distal M2-aneurysm, as well as a voluminous right MCA-aneurysm with a significant distal M2 aneurysm: first, the left-sided aneurysms were clipped under HCCA using IOA following which she did well in spite of persistent vasospasm documented by TCD and successfully reversed with Triple $\mathrm{H}$ therapy. The right-sided aneurysms were operated on six months later with temporary clipping and in spite of IOA she developed a secondary partial thrombosis of one distal M2 branch which resulted in a largely reversible stroke and was treated with Triple $\mathrm{H}$ therapy as well.

The second patient, a 47 year old hypertensive lady was investigated because of left internal carotid occlusion and was found to have a voluminous unruptured basilar apex aneurysm with a large neck: using HCCA, orbitozygomatic craniotomy, extensive mobilization of the optic nerve and carotid ophthalmic segments of the ICA, the aneurysm was clipped successfully. Post-operatively, she also developed signs of clinical vasospasm with reversible hypodensities in the left posterior cerebral 
territory and both thalami which improved remarkably after aggressive hyperdynamic therapy (Triple $\mathrm{H}$ ).

The indications of HCCA as well as of IOA and the occurrence of post-operative vasospasm in unruptured aneurysm will be discussed.

\section{$12-16$}

\section{Multimodality Management of Giant and Complex Unruptured Aneurysms}

G.Mohr, D. Tampieri (Montreal, Quebec)

The authors present a series of 18 procedures performed on 15 patients, including 1 petrous, 2 cavernous, 5 paraclinoid, 7 MCA and 3 basilar apex aneurysms with either giant or complex features. Surgical treatment consisted of standard clipping in 5 cases, 2 of which were done under deep hypothermia and total cardio-circulatory arrest, excision and clipping in 2 cases and 2 cases with paraclinoid aneurysms required combined intra and extracranial trapping, and ECIC-bypasses were used in 3 cases. Intraoperative angiography was performed in 5 cases. Endovascular treatment ws applied in 9 cases including 5 GDC platinum coil embolization, 1 failed attempt at coiling and 3 cases with balloon trapping or occlusion.

No mortality occurred in these series. Following surgical treatment, 3 patients out of 9 experienced ischemic complications including one small embolic infarct following trapping, one M2-branch occlusion following clipping of MCA aneurysm with mild deficit and one thalamo-perforator damage with moderate incapacitation after hypothermic cardiac arrest for large basilar apex aneurysm. One patient developed post-op intracerebral hematoma treated conservatively with total recuperation. Following endovascular treatment, only 1 patient with coiled carotid-ophthalmic aneurysm suffered partial retinal ischemia and two attempts at coiling failed, necessitating balloon-trapping in one case. Only one patient with basilar aneurysm had a residual neck which has remained stable at one year angiogram.

\section{2-17}

Intracranial Aneurysm Surgery Performed in a Postgraduate Training Programme

S Woodrow, M Tymianski, F Gentili, MC Wallace (Toronto, Ontario)

Background: Postgraduate training for neurosurgeons in aneurysm surgery requires supervised "hands-on" education in the operating room. Quality of care must be prioritized over the delegation of appropriate surgical responsibility in the theatre. This study was designed to evaluate the outcome of patients treated surgically for intracranial aneurysms at a teaching centre.

Methods: All surgical cases $(\mathrm{n}=260)$ for aneurysm from 1993-1997 were selected. The operating surgeon and assistant were identified. Follow-up information involved examination in clinic and determination of Glasgow outcome scale.

Results: Prospective information was collected in only
140/260 cases, despite available forms. Dissection of the aneurysm was performed by the resident in $66 \%$ of cases and the fellow $34 \%$. 33/140 (24\%) patients were asymptomatic. The attending staff became involved and clipped the aneurysm in 14 $/ 140(10 \%)$ cases. There was no difference in operating surgeon between ruptured and asymptomatic aneurysms. $82 \%$ were WFNS grades $1-3$ and $89 / 117(76 \%)$ had excellent outcome at mean follow-up 101 weeks. Overall mortality was $9 / 140(6 \%)$. In the 120 patients where the responsible surgeon was not recorded prospectively, similar outcome results were obtained.

Conclusion: With careful supervision, intracranial aneurysm surgery can be safely delegated to neurosurgical trainees in their senior years, with good outcome.

\section{2-18}

Hypothermic Circulatory Arrest in the Treatment of Intracranial Aneurysms: The University of Alberta Experience

J.M. Findlay, D.E. Steinke, B.E. Marchak, J.C. Mullen, E.T. Gelfand, A. Koshal (Edmonton, Alberta)

Introduction: The use of cardiopulmonary bypass and the induction of profound hypothermia with circulatory arrest reduce the tension and size of intracranial aneurysms, and in some patients may allow safer dissection and repair of large intracranial aneurysms. We describe the methods and results of this technique as performed at the University of Alberta.

Materials and Methods: In the past six years, 6 patients ages 36 to 74 years, have undergone the open-chest method of hypothermic circulatory arrest for the repair of 2 basilar apex and 4 internal carotid artery (ICA) large to giant aneurysms (mean aneurysm diameter $24 \mathrm{~mm}$ ). One giant ICA aneurysm presented with headache and visual loss, and the other 4 aneurysms had bled ( 3 patients Botterell-Lougheed grade 4, and 2 patients grade 2). No patient had a history of serious heart disease.

Results: The operating time intervals were as follows (range and mean, in minutes) time to cool to $16-20^{\circ} \mathrm{C} 30-74,46$; arrest time 7-28, 19; rewarming to $36^{\circ} \mathrm{C} 55-120,76$; total pump time 120-199, 145; total operating time $280-360,317$. Circulatory arrest and partial exsanguination were considered useful in every case except one, where several large arterial branches arising from the aneurysm dome (including the anterior choroidal artery) prevented arterial reconstruction, and parent artery occlusion was performed. There were no complications due to bypass. Long-term outcome has been good (Glasgow Outcome Scale) in every patient except the last, mentioned above, where deliberate parent artery (left ICA) occlusion led to aneurysm thrombosis associated with cerebral infarction and severe disability at three months follow-up.

Conclusions: In our experience to date, circulatory arrest and partial exsanguination under neuroprotective profound hypothermia have been useful adjuncts to the definitive repair of most large to giant intracranial aneurysms we have selected for this technique, and complications due to this procedure alone (i.e. hemorrhagic or cardiac) have not occurred. 


\section{TRAUMA}

13-01

Concussion in the Elite Athlete: Neuroimaging and Neuropsychological Correlates

K. Johnston, S. Leclerc, V. Lacroix, M. Guerin, R. delCarpio, M Lassonde, A. Ptito, S. Delaney, C. White D. Mulder (Montreal, Quebec)

Background: Considerable controversy exists with respect to the grading of concussion and return to play guidelines. As a result, efforts to scientifically define and evaluate concussion from both neuroimaging and neuropsychological perspectives are underway. A review of recent available data in the literature including SPECT and PET studies, will be presented and our current imaging and neuropsychological study introduced.

Methods: Evaluation of injured athletes was performed and concussions graded according to the McGill System. Serial follow up and neuropsychological testing was carried out according to specific protocol. Athletes with ongoing symptoms were further evaluated using the following imaging techniques; CT, MRI, flair MRI, diffusion MRI +/- PET and fMRI. In this pilot study, findings were correlated with concussion grade, clinical course and neuropsychological findings.

Results: Use of specific on field and follow up neuropsychological testing corresponded well with concussion grade and neuroimaging results. Results accurately predicted clinical course. Effects on long term sequelae are still undetermined.

Conclusion: While CT and routine MRI offer few findings in athletic concussion, newer imaging techniques in combination with specific neuropsychological tests may have potential in predicting clinical course and outcome.

\section{3-02}

\section{McGill Guidelines for Concussion Grading and Return to Play}

K. Johnston, S. Leclerc, V. Lacroix, S. Delaney, D. Kinnear, C. White, D. Mulder (Montreal, Quebec)

Background: Sports related concussions are a common and important cause of injury in the elite athlete. These head injuries vary in severity from "mild" to "severe". Corresponding sequelae related to the grading of concussion have led to controversy. Given recent new information in the clinical and neuropsychological literature, it is our mandate to develop clinically useful guidelines for grading and return to play. Current grading systems will be reviewed and the new McGill grading system described.

Methods: A critical review of existing literature and guidelines was undertaken and on this basis the McGill guidelines were proposed. These guidelines were then evaluated in a pilot study to determine if they were useful in predicting clinical course, neuroimaging and neuropsychological correlates, and return to play decisions.

Results: New guidelines will be described incorporating existing guidelines including those of Cantu, AAN and Colorado. One salient difference between the new guidelines and those previously published is in the subdivision of Grade 1 concussion into $\mathrm{A}, \mathrm{B}$ and $\mathrm{C}$ with resulting differences in presentation and clinical course.

Conclusions: The McGill guidelines are useful in predicting clinical presentation and course and as such now form the basis for our prospective study to describe neuropsychological and neuroimaging correlates of sports related concussion.

\section{3-03}

\section{ICP Monitors in an Artificial Brain Model}

\section{R Akagami (Vancouver, British Columbia)}

Background: Occasionally there is disparity in the readings between an external ventricular drain (EVD) and a Camino fiberoptic intraparenchymal monitor when placed in the same patient. There is difficulty in using cadaveric material and there are no artificial brain models to study mass lesions and monitoring devices. An artificial brain model was developed and the effects of a simulated frontal mass lesion observed using bilateral EVD's and Camino monitors placed at various positions and depths in the frontal lobes.

Methods: Powdered agar-agar was chosen for its consistency and its ability to remain set at room temperatures. Anatomically correct lateral ventricles were carved from a block of ice using a high speed drill. Liquid agar-agar was poured into a plastic skull over frozen ventricles. Once set, an artificial brain with fluid lateral ventricles is created. Bilateral EVD's and a Camino were used. Silicon was injected as a frontal mass. Camino depth and distance from the superior sagittal suture were varied.

Results: EVD values on either side were within $5 \%$ of each other. Camino depth at $0.5 \mathrm{~cm}$ consistently gave lower values compared with EVD. In small lesions $(<12 \mathrm{cc})$, varying the position or depth of the Camino made little difference in values. With 45cc lesions, Caminos placed ipsilateral to the lesion gave similar readings to EVD's; but, there was reproducible disparity between the EVD's and Caminos placed contralateral to the lesion. The Camino values approached EVD readings as the monitor depth was increased and placed further from the sagittal suture.

Conclusion: A relatively anatomically correct artificial brain model can be constructed. In this model there are differences in pressures between EVD's and Caminos placed contralateral to lesions. Camino values reflected EVD values better if placed deeper that the standard $0.5 \mathrm{~cm}$ position and if used ipsilateral to the lesion. When placed contralateral, it should be placed 1.5 to $2.5 \mathrm{~cm}$ deep and $>4 \mathrm{~cm}$ lateral to the sagittal suture. Further work is required to make physical measurements on the model to compare with the living brain. Although this model is totally artificial, there are similarities in general construction and some implications can be made about the real system. With some modifications, this model may enjoy some other uses in looking at other forms of injury and practice with endoscopic systems. 
13-04

\section{Mortality Following Minor Head Injury: The 'Talk and Die' Patient Revisited}

\section{Belyea, R. Moulton (Toronto, Ontario)}

To examine the cause of potentially preventable deaths following head injury we reviewed 30 patients who died following minor head injuries (admission GCS 13-15) with respect to patient and injury characteristics as well as timing of diagnosis and surgery.

Of 2310 patients with head injuries of all degrees severity admitted to St. Michael's Hospital over a 10 year period we identified 1183 patients admitted with a GCS score of 13-15. Thirty of these patients died (2.5\%). Eight patients died of causes other than their incidental minor brain injuries. The causes of death were acute exsanguination or delayed multiple system organ failure related to systemic injuries. Of the 22 patients dying of their brain injuries, 16 had operable cerebral mass lesions (13 acute subdural hematomas, 3 intracerebral hematomas/contusions). The mean age of these 22 patients was 70 yrs (range 24 - 93). No operation was undertaken in 3 of the sixteen patients with mass lesions. Of the remaining 13 patients surgical management was deliberately delayed (patients kept under observation) in four (mean time to OR $141 \mathrm{hrs}$.). Of the nine patients operated on immediately after diagnosis, operation was undertaken within 4 hours of admission in 5 and within 4.5 hours in another 2 patients. In one 24 year old patient there was a delay in the diagnosis of an intracerebral hematoma of 8 hours and a delay in surgical evacuation of 10 hours. Mean time to death in the 22 patients dying as a result of their brain injury was 17 days.

The majority of patients dying after minor head injury were elderly, and died of delayed medical complications of their injuries. Prompt diagnosis and surgical evacuation of their hematomas did not appear to impact on their outcome. This suggests mortality in the 'talk and die' patient population may not be as easily reduced as implied by earlier work in this field.

\section{PEDIATRIC NEUROSURGERY}

\section{4-01}

\section{Neurosurgical Aspects of the Management of Neonatal} Alloimmune Thrombocytopenia

\section{BS Jhawar, RF Del Maestro (London, Ontario)}

Background: Neonatal Alloimmune Thrombocytopenia (NAT) is a not uncommon disorder of fetal-maternal platelet incompatibility. The disorder results from a maternal antibody response against paternal antigens found on fetal platelets. The potential for profound thrombocytopenia can result in intracranial hemorrhage in up to $20 \%$ of cases.

Methods: We retrospectively reviewed all charts over the decade preceding 1997 with the diagnosis of NAT. Clinical and laboratory records, imaging studies and follow-up data were analyzed. Current status of each infant was determined by telephone interview.

Results: A total of 66 full-term infants diagnosed with intracranial hemorrhage were managed at our institution. Ten infants were diagnosed with NAT based on platelet antigen testing. Hemorrhage tended to be severe (severe 4 , moderate 5 , and mild 1). One child required a VP shunt while another was managed with serial ventricular taps. There was one death. Minor long-term physical or cognitive disability occurred on all but three infants.

Conclusions: Intracranial hemorrhage is an important cause of perinatal mortality and potential lifelong morbidity. In the management of hemorrhage due to NAT, it is paramount to correct thrombocytopenia. Medical management usually involves platelet transfusion, corticosteroids and intravenous gamma globulin. Neurosurgical care involves the early identification of hemorrhage, control of hydrocephalus and potentially operative intervention. The increased risk for such events in subsequent pregnancies must be recognized and the appropriate precautions should be taken.

\section{4-02}

\section{Neuroleptic Anaesthesia In Children With Cerebral Lesions}

R.F. Del Maestro, M. Austin (London, Ontario)

Background: The surgical treatment of cerebral lesions in and/or near eloquent brain regions is associated with increased patient morbidity. We report our experience with 11 children who underwent 15 operations under neuroleptic anaesthesia in an attempt to decrease patient morbidity:

Methods: The scalp was anaesthesized with $0.5 \%$ Marcaine and 1/200 epinephrine and neuroleptic anaesthesia was induced and maintained using Fentanyl, Midazolan and Propofol. Cortical stimulation was used as required.

Results: Eight patients had cerebral tumors, four glioblastoma multiforme, one each with astroblastoma, ependymoma, pilocytic astrocytoma and germinoma. The majority of these children presented with increased intracranial pressure (8) and one had seizures. One patient presented with a cerebral hemorrhage secondary to an AVM, one with seizures and a fungal abscess and one patient presented with increased intracranial pressure and seizures and a final diagnosis has not been established. The median age of the patients was 11 (range 8 - 18) with median operative time of 6 hours (range $4-9$ ) and the median hospital stay was 5 days ( $3-8)$. No difficulties were encountered with the induction and/or maintenance of neuroleptic anaesthesia and no patient suffered seizures or needed to be intubated during the procedure. The three operative indications for the use of neuroleptic anaesthesia were: 1) The maintenance of speech and/or motor function ( 6 patients, 8 operations). 2) To allow for occlusion and/or excision of major vessel during the resection (4 patients, 5 operations). 4) To preserve visual function (1 patient). Five patients develop an intraoperative change in function which resulted in a change in operative plan. In two patients motor dysfunction resolved by the end of the procedure, another by discharge and two have minimal long term dysfunction. Gross 
removal of the enhancing mass was accomplished in 10 of 13 operations and the AVM was completely excised. No post-operative complications were encountered.

Conclusions: 1) Neuroleptic anaesthesia provides the surgeon with another surgical option for the pediatric patent which may result in less post-operative morbidity and more complete excision of the lesion. 2) Age does not seem to be a significant problem in being able to carry out these procedures.

\section{GENERAL NEUROSURGERY}

\section{5-01}

Evaluating the Internet as a Source of Medical Information. Are Your Patients Well Informed?

B.M. Wheatley, J.M. Findlay, D.E. Steinke (Edmonton, Alberta)

Background: In an attempt to obtain information on the nature and treatment of their illnesses, patients increasingly turn to the Internet as a source of medical information. However the Internet is presently an unsolicited and unverified source of information

Methods: To measure the quality of information available on the Internet, we used 5 popular search engines (Yahoo, AltaVista, Excite, Hot Bot and Webcrawler) to search the world wide web for several specific neurosurgical problems: arteriovenous malformations, malignant astrocytomas and meningiomas. The top 50 web sites for each search engine were visited and graded according to the relevance and quality of the information as follows: Grade 1 - unrelated site; Grade 2 - no useful information (e.g. anecdotes, physician curriculum vitaes and simple bibliographies); Grade 3 - partial information without disclosure of all treatment options (e.g. case reports); Grade 4 - full discussion of subject with all treatment options but without references; Grade 5 - full discussion of the subject with references.

Results: The number of web sites found by each search engine ranged from $4-1024$. Thirteen percent of web sites were found to be grade 4 or 5 . Sixty five percent of web sites were unrelated to the topic or had no useful information. The quality of the web sites identified by each search engine was variable with average grades ranging from 1.9 for AltaVista to 3.0 for Yahoo.

Conclusion: Internet searches for medical information results in a large number of poor quality sites. In spite of the low yield for high quality sites, those sites with grades of 4 or 5 are excellent sources of information (for the topics we examined) for patients and their families. High quality web sites will be identified.

\section{5-02}

Septic Cavernous Sinus Thrombophlebitis, Cerebellar Abscess and Subdural Empyema, and Pituitary Abscess Secondary to Pansinusitis-Case Report

Ramesh L. Sahjpaul, Don H. Lee (London, Ontario)

A 26-year-old male with a past history of paranasal sinusitis developed pansinusitis with subsequent bilateral septic cavernous sinus thrombophlebitis, infratentorial empyema, a pituitary abscess, and a rapidly progressive clinical deterioration. Aggressive medical (intravenous antibiotics) and surgical (paranasal sinus drainage and drainage of the cerebellar abscess and subdural empyema) resulted in survival of the patient. Residual deficits were limited to panhypopituitarism and monocular visual impairment.

Sphenoid sinusitis, septic cavernous sinus thrombophlebitis, pituitary abscess and infratentorial empyema are all uncommon clinical entities and the concurrent occurrence of all has not previously been reported. This unusual case serves to emphasise the potential serious intracranial complications of paranasal sinusitis and the role of aggressive medical and surgical treatment in this condition.

\section{5-03}

Pulsating Exophthalmos Caused by Orbital Roof Arachnoid Diverticulum: Case Report

B. Addas, D.B. Clarke, V.E. Sangalang, R. Vandorpe, J. MacNeill (Halifax, Nova Scotia)

Background: Pulsating exophthalmos is an uncommon symptom in neurosurgery, usually attributable to one of several pathologies, including carotid-cavernous fistula, vascular malformation and neoplasm of the orbit with transmission of cerebral pulsations through a defect in the orbital roof.

Methods: The authors describe a previously unreported cause of pulsating exophthalmos: orbital roof arachnoid diverticulum.

Results: Pre-operative neuroradiological investigation demonstrated a bony defect in the left orbital roof and herniation of a cerebrospinal fluid (CSF)-filled sac into the orbit; the brain was normal in appearance. A left frontal craniotomy revealed a CSF sac in continuity with the subarachnoid space and herniating through an eroded orbital roof. Reconstruction of the patient's orbital roof resulted in complete resolution of the pulsating exophthalmos. Pathological examination of the coverings of the diverticulum showed a thinned dural membrane with an inner lining of arachnoid, consistent with an arachnoid diverticulum.

Conclusion: The authors present a previously unreported case of pulsating exophthalmos caused by an arachnoid diverticulum that was successfully treated with craniotomy and orbital roof reconstruction.

\section{5-04}

\section{Correlation of B Waves in Normal Pressure Hydrocephalus}

N. Khairallah, Pascale Demers, Alain Robillard, J Teitelbaum (Montreal, Quebec)

The interpretation of data from continuous monitoring of intracranial pressure (ICP) in patients with suspected normal pressure hydrocephalus (NPH) is the subject of controversy. Despite the fact that overnight ICP monitoring is widely used for the diagnosis of NPH, normative criteria are poorly defined. 